\title{
WestVirginiaUniversity
}

THE RESEARCH REPOSITORY @ WVU

Graduate Theses, Dissertations, and Problem Reports

2017

\section{A New Compactification for Celestial Mechanics}

\author{
Daniel Solomon
}

Follow this and additional works at: https://researchrepository.wvu.edu/etd

\section{Recommended Citation}

Solomon, Daniel, "A New Compactification for Celestial Mechanics" (2017). Graduate Theses,

Dissertations, and Problem Reports. 6689.

https://researchrepository.wvu.edu/etd/6689

This Dissertation is protected by copyright and/or related rights. It has been brought to you by the The Research Repository @ WVU with permission from the rights-holder(s). You are free to use this Dissertation in any way that is permitted by the copyright and related rights legislation that applies to your use. For other uses you must obtain permission from the rights-holder(s) directly, unless additional rights are indicated by a Creative Commons license in the record and/ or on the work itself. This Dissertation has been accepted for inclusion in WVU Graduate Theses, Dissertations, and Problem Reports collection by an authorized administrator of The Research Repository @ WVU.

For more information, please contact researchrepository@mail.wvu.edu. 


\title{
A New Compactification for Celestial Mechanics
}

\author{
Daniel Solomon \\ Dissertation submitted \\ to the Eberly College of Arts and Sciences \\ at West Virginia University \\ in partial fulfillment of the requirements for the degree of
}

Doctor of Philosophy in

Mathematics

\author{
Harry Gingold, Ph.D., Chair \\ Harvey Diamond, Ph.D. \\ Leonard Golubovic, Ph.D. \\ Harumi Hattori, Ph.D. \\ Dening Li, Ph.D. \\ Department of Mathematics \\ Morgantown, West Virginia \\ 2017
}

Keywords: escape in celestial mechanics, compactification, singular points of differential equations, critical points at infinity, Lorenz system, completeness, asymptotic analysis, degenerate critical points, expanding gravitational systems 


\title{
ABSTRACT \\ A New Compactification for Celestial Mechanics
}

\author{
Daniel Solomon
}

This is an exposition of a new compactification of Euclidean space enabling the study of trajectories at and approaching spatial infinity, as well as results obtained for polynomial differential systems and Celestial Mechanics. The Lorenz system has an attractor for all real values of its parameters, and almost all of the complete quadratic systems share an interesting feature with the Lorenz system.

Informed by these results, the main theorem is established via a contraction mapping arising from an integral equation derived from the Celestial Mechanics equations of motion. We establish the existence of an open set of initial conditions through which pass solutions without singularities, to Newton's gravitational equations in $\mathbb{R}^{3}$ on a semi-infinite interval in forward time, for which every pair of particles separates like At, $A>0$, as $t \rightarrow \infty$. The solutions are constructible as series with rapid uniform convergence and their asymptotic behavior to any order is prescribed. We show that this family of solutions depends on $6 N$ parameters subject to certain constraints. This confirms the logical converse of Chazy's 100-year old result assuming solutions exist for all time, they take the form given by Bohlin: solutions of Bohlin's form do exist for all time. An easy consequence not found elsewhere is that the asymptotic directions of many configurations exiting the universe depend solely on the initial velocities and not on their initial positions.

The N-body problem is fundamental to astrodynamics, since it is an idealization to point masses of the general problem of the motion of gravitating bodies, such as spacecraft motion within the Solar System. These new trajectories model paths of real particles escaping to infinity. A particle escaping its primary on a hyperbolic trajectory in the Kepler problem is the simplest example. This work may have relevance to new interplanetary trajectories or insight into known trajectories for potential space missions.

I know that the tide is not an independent force, but merely the submission of the water to the movement of the moon in its orbit. And this in turn is subject to other orbits which are mightier far than it. And so the whole universe is held fast in the clinging grip of strong hands, the forces of Earth and Sun, planets and comets, and galaxies, blindly erupting forces ceaselessly stirring in ripples of silence to the very depth of black space. 


\section{DEDICATION}

This work is dedicated to my children and grandchildren, without whom it would have been finished many years earlier; I am proud of them every day. It is also dedicated to the memory of Charles Conley, a pioneer in the use of cohomology methods for dynamical systems [21]. I am grateful that he interested me in dynamical systems.

In one sense this dissertation is the completion of a 41-year effort, since I started grad school in 1976. On the other hand, I come from a family of late bloomers. My father left aerospace engineering and was ordained at the age of 59. He held a pulpit before retiring again in his 70s and along the way he earned an MA in Theology at 63 . Now at 86 , he continues to audit advanced courses in math and physics at a nearby university. While raising five sons my mother finished college at 47 and then law school at 58, in just four years while working full time. After retiring from her job clerking for an administrative law judge at 60 , she opened her own legal practice until retiring again at 72. My stepmother left music education at 59, and at 80 continues to publish on the subject, teach workshops in the US and around the world, direct a choir, and perform piano concerts in New York. My oldest brother was an Army officer when he began medical school, from which he graduated at 45. He became a neurologist and was recently promoted to full Colonel in the Medical Corps. Our middle brother has a successful career in the banking industry, but he cannot escape the need to play the blues on a slide guitar, which he started at 45. My next brother is an Israeli tour guide and educator based in Jerusalem. While not a late bloomer, he has one of the coolest jobs in one of the most special places in the world, and he lives a few hundred meters from where our grandfather was born. My youngest brother (also a lawyer) built an airplane in his garage. After ten years of work on the plane, it was certified for flight when he was 44 .

At almost 64 , I'm right on time.

If I had not lived until I was 90, I would not have been able to write this book. ... It could not have been done even when I was ten years younger. I wasn't ready. God knows what other potentials lurk in other people, if we could only keep them alive well into their $90 \mathrm{~s}^{1}$

- Harry Bernstein

\footnotetext{
${ }^{1}$ Quoted in Senior Wonders [81], a sixtieth birthday present from my wife. Mr. Bernstein was awarded a Guggenheim Fellowship in 2008 to further pursue his writing (at 98!) after the publication of two well-received autobiographical novels, The Invisible Wall (2007) and The Dream (2008). It worked, too; his final novel The Golden Willow was published in 2009.
} 


\section{ACKNOWLEDGEMENTS}

Much of this work was supported by NASA's academic program. NASA is very generous with training, and it has been very generous with me!

In addition, I would like to express my gratitude to many people for a wide variety of help and support:

- A very special acknowledgement goes to my wife, Daya. I am beyond grateful for the life and love that we share.

- My parents, who wakened my interests in math and science.

- Three teachers from Moorestown Junior High School that encouraged me: Charles Barnosky, Florence Kerr, and Andrea Rittenhouse.

- Adam Bincer, from whom I learned to solve hard problems. He gave incredibly difficult homework problems in his courses in Quantum Mechanics, Quantum Electrodynamics, and Relativistic Quantum Electrodynamics. Since they were homework, one knew (or at least assumed) that they could be solved, and this was enough incentive for me to keep at it until the problems were solved.

- Bernice and Randy Durand, who took me in a little bit while we studied topological methods in high energy physics together.

- I thank my bosses for committed support: Carl Adams, Tupper Hyde, Juan Roman, Karen Flynn and Dennis Andrucyk. I still owe Karen a dollar.

- Harry Gingold - enormous thanks is due to my long-suffering advisor. I am grateful for the opportunity to study under and work with an expert in asymptotic methods and singular problems.

- A book that I've read more often than any other is Ursula K LeGuin's The Dispossessed, which I read whenever I feel in need of inspiration. In addition, I treasure a post card she sent me in response to my fan mail. 


\section{Contents}

\section{ABSTRACT}

DEDICATION

\section{ACKNOWLEDGEMENTS}

List of Figures

Chapter 1. Introduction

1.1. Celestial Mechanics, Singularities, and Infinity

1.2. Compactifications, Old and New

1.3. The Flow on the Boundary and at $\infty$

1.4. Notation Glossary and Key Definitions

Chapter 2. Applications to the Lorenz and Other Polynomial Systems

2.1. The Lorenz System has a Global Repeller at Infinity

2.1.1. Compactification and Behavior at Infinity

2.1.2. Proof of Repulsion

2.2. Completeness of Quadratic Systems

2.2.1. Completeness and Structure of Lorenz-like Systems

2.2.2. Some incomplete systems

2.3. Fields of Lorenz-like Systems Near $\infty$

Chapter 3. Celestial Mechanics at $\infty$ via Compactification

3.1. Compactifying the N-Body Problem

3.1.1. Time derivatives

3.1.2. Including infinity

3.1.3. Seeking a series expansion

3.2. The System on the Boundary Sphere

3.3. Approximate Solutions near the Boundary

3.3.1. An approximating system

3.3.2. A closer approximation

Chapter 4. Seeking Trajectories that Expand to the Boundary

4.1. Perturbation near an approximate solution

4.2. The Linear System for $\Delta$

4.2.1. Solving the $J_{0}$ Problem

4.2.2. The $J_{1}$ Problem

4.3. Return to the Uncompactified Problem

Chapter 5. Solutions That Escape

5.1. Seeking solutions with escaping trajectories

5.2. Estimates for $P 1$ and $P 2$

5.2.1. Estimating $P 1$

5.2.2. Estimating $P 2$

5.2.3. Estimating $P 2(\delta)-P 2(\eta)$ in terms of $\delta-\eta$

5.3. The integral equation iii

iv

vii

1

3

6

9

12

15

15

16

18

20

21

23

25

31

31

34

36

39

42

46

47

50

58

58

66

66

70

75

78

79

80

81

84

86

87 
5.4. Comparisons with previous work

Chapter 6. For Further Investigation

6.1. Rescaled time

6.2. Compactify in Position Only

98

6.3. Analyze $1-R^{2}$ as a Bernoulli equation

6.4. Celestial Mechanics with a Forcing Term

100

6.4.1. Seeking a Power Series for $q_{i}$

102

6.4.2. Compactifying the Forced N-Body Problem

104

Bibliography

106 


\section{List of Figures}

1.2.1 Compactification of $\mathbb{R}^{2}$

3.2.1 Boundary trajectories tending to the critical point set. The two singular points $\left\{x_{1}=x_{2}\right\}$ in the critical set $C P=\mathbb{S}^{1}$ are marked as o.

3.3.1 Illustrating Proposition 27 


\section{CHAPTER 1}

\section{Introduction}

The differential equation governing the position 3-vectors $q_{i}$ of $N$ point-masses $m_{i}, i=1, \ldots, N$ moving in $\mathbb{R}^{3}$ under the influence of their mutual gravitation is

$$
\ddot{q}_{i}=\sum_{j \neq i} \frac{m_{j}\left(q_{j}-q_{i}\right)}{\left\|q_{i}-q_{j}\right\|^{3}}=\frac{1}{m_{i}} \frac{\partial U}{\partial q_{i}},
$$

where conventionally $U=\sum_{j<k} \frac{m_{j} m_{k}}{\left\|q_{j}-q_{k}\right\|}>0$ is the negative of the potential energy, and the units are chosen so that the universal gravitational constant is 1 . We demonstrate the existence of a multi-parameter continuum of trajectories escaping to infinity as $t \rightarrow \infty$ in many possible sets of directions in $\mathbb{R}^{3}$. Moreover, they take the form suggested by Chazy [17], which he credits to Bohlin [13]

$$
q_{i}=a_{i} t+b_{i} \log t+c_{i}+\delta_{i},
$$

where each $a_{i}, b_{i}$ and $c_{i}$ is a constant 3 -vector, the $a_{i}$ and $c_{i}$ are nearly arbitrary, $b_{i}$ depends on the $a_{i}$, and each 3 -vector $\delta_{i}$ is shown to tend to 0 . We provide asymptotic approximations and rapidly-converging series for $\delta_{i}$. Chazy [17] showed that if there are solutions that exist for all time, and if the energy $h$ is positive, the solutions must take the form (1.0.2). Until now, no one had proved that such solutions actually exist.

Under the compactification described in Section 1.2, a set of particles approaching infinity in a given set of directions becomes a trajectory in the unit ball of $\mathbb{R}^{6 N}$, approaching a critical point of the compactified flow on its boundary, the unit sphere $\mathbb{S}^{6 N-1}$. As we will see, the critical point set is degenerate, a $3 N$ 1-sphere, contained within the boundary. The flow on the boundary is quite simple, and we describe it in Chapter 3. We will see how the flow on the boundary gives insight into the flow of the uncompactified system for large $\left\|q_{i}\right\|$ or $\left\|\dot{q}_{i}\right\|$. In many cases the Jacobian at critical points at infinity can provide information on the completeness of the uncompactified problem. However, for Celestial Mechanics the Jacobian of the compactified flow is nilpotent at every critical point. Thus all eigenvalues are 0 , and the Jacobian does not tell us whether the critical points attract. Despite the lack of information from the Jacobian, we are able to show that many critical points within the boundary do in fact attract a multi-dimensional continuum of points. Moreover, the flow on the boundary tends to the same critical point set, and boundary trajectories provide asymptotic information about escaping trajectories in celestial mechanics.

This Chapter provides the background and context for this work; we discuss escape to infinity in celestial mechanics, introduce the compactification published for polynomial systems, and summarize results obtained for polynomial systems. As we will show, our compactification of Euclidean space $\mathbb{R}^{n}$ is its open unit ball $\mathbb{B}^{n}$, the pre-image of whose boundary sphere $\mathbb{S}^{n-1}$ (under the compactification) may be viewed is an ideal set at infinity in an extended $\mathbb{R}^{n}$, represented by all possible directions of the sphere. The compactification of a differential equation defined on the interior of unit ball extends to its boundary, which can prove fruitful. The behavior of the system on the boundary sphere is simpler and often illuminates asymptotic analysis. Indeed, these ideal solutions provide a wealth of information on the solutions of $\dot{y}=f(y)$ for $\|y\|$ large. This Chapter includes a survey of relevant literature and concludes with notation conventions.

This compactification method was proposed by Gingold in [38] and was applied to polynomial systems by Elias and Gingold in [35]. It provides a better understanding of the behavior of solutions of dynamical systems when the norm of the solutions is large and becomes unbounded. It augments the arsenal of methods like Lyapunov functions, the Poincaré compactification, and the Poincaré map that are used to prove global existence of solutions and completeness of dynamical systems.

The compactification described and used here has advantages over the more commonly used stereographic projection of Bendixson [10], as well as that of Poincaré [85] (see also the Perko text [83] for an exposition): our compactification distinguishes directions at $\infty$ and transforms rational ordinary differential equations 
to rational ordinary differential equations. The development of the compactification in [38] and [35] was influenced by the work of Gingold and Gingold [39], where the stereographic projection is obtained as a degenerate limit of a family of compactifications that account for "all directions at infinity." However, that compactification is akin to the Poincare compactification and possesses radicals that prevent it being a tool for rational approximations.

Chapter 2 presents some results obtained on compactifying certain polynomial systems. I compactified the Lorenz system for a wider set of parameters $\sigma, \rho, \beta$ than is usually treated and proved that it has a global repeller at infinity. I calculated the asymptotic behavior of the vector fields $T, N, B$ of differential geometry for a class of quadratic systems in $\mathbb{R}^{3}$ including the Lorenz system for all values of its parameters and its compactification, showing that they coincide to lowest order. On compactifying quadratic systems in general, I gave a characterization of completeness. This work was published in three papers with Gingold $[40,41,42]$. Here as well, despite a Jacobian whose eigenvalues are all 0 at the critical points, we are able to show that the boundary sphere for the Lorenz system repels trajectories that start near the boundary.

In Chapter 3, we extend the compactification of Section 1.2 beyond polynomial systems to the equations of celestial mechanics, analyze the behavior of the compactified system in the boundary, and investigate solutions to some approximate systems near the boundary. The compactified system can be extended to the non-collision boundary points, and and we show in fact that all critical points and singular points of the system are on the boundary sphere. Next we study the system restricted to the boundary sphere, where the behavior is so much simpler that exact solutions can be given in closed form. These solutions represent new objects in Celestial Mechanics, as the compactification of trajectories at $\infty$. In the absence of collisions, these solutions on the boundary approach the critical point set, which is the unit $3 N-1$-sphere where $x_{N+i}=0$. These solutions offer insight into solutions near the boundary, in particular suggesting a set of approximating systems near the boundary. We determine properties of solutions to two approximating systems, both of which can be solved exactly, and one of these solutions is asymptotic to solutions of the compactified system. The uncompactification of the second approximate solution will be shown to be asymptotic to the new solutions we give in Chapter 5 to Celestial Mechanics equation.

Given a solution $x$ and an approximation $\bar{x}$, we develop in Chapter 4 the differential equation satisfied by the difference $\Delta=x-\bar{x}$. We will be interested in decaying solutions $\Delta$, whose existence guarantees that $x$ and $\bar{x}$ are asymptotic to the same point $\left(x^{* \dagger} 0_{3 N}^{\dagger}\right)^{\dagger}$ as $t \rightarrow \infty$, and we can interpret the unit $3 N$-vector $x^{*}$ as the $N$ limiting directions $x_{i}^{*}$ of the particles. To better understand the problem for $\Delta$, which has an irregular singular point at $t=\infty$, we begin with efforts to solve approximating versions of the problem, seeking solutions to the linear problem that decay to 0 . After a very helpful coordinate transformation, we solve several approximate problems and find solutions tending to 0 . This work with the linear system inspires our search for solutions to the full problem in the next Chapter, returning to the uncompactified problem and the selection of a specific form of solution to pursue.

Chapter 5 establishes the main theorem of the dissertation, that solutions of Bohlin's form (1.0.2) do indeed exist for all time. We demonstrate the existence of an open set of initial conditions through which pass solutions without singularities, to Newton's gravitational equations in $\mathbb{R}^{3}$ on a semi-infinite interval in forward time, for which every pair of particles separates like At, $A>0$, as $t \rightarrow \infty$. The solutions are constructible as series with rapid uniform convergence and their asymptotic behavior to any order is prescribed. This family of solutions depends on $6 N$ parameters subject to certain constraints. The key to the result is converting the differential equation for $q$ into an integral equation for $\delta$ and showing that integral equation has a solution. The results of this Chapter were published in 2017 [43].

In Chapter 6, we provide some ideas of directions that this research could be extended. These include

- How and why one might rescale time for the Celestial Mechanics problem, as is done for polynomial systems in Section 1.2 and Chapter 2

- Compactifying the Celestial Mechanics problem in position only, rather than in $y=\left(\begin{array}{c}q \\ \dot{q}\end{array}\right)$

- Studying the behavior of $1-R^{2}$ near the boundary as a Bernoulli problem

- Considering the forced Celestial Mechanics problem

as well as compactifying the ambient $\mathbb{R}^{3}$, rather than phase space; extending the concept to the motion of charged particles subject to their mutual electromagnetic attraction/repulsion; application of the Conley Index [21] to study orbits that connect to the invariant set on the boundary; applications of compactification 
to partial differential equations; and applications of the methods and results to astronomy and aerospace engineering.

\subsection{Celestial Mechanics, Singularities, and Infinity}

In this Section, we describe the state of knowledge of completeness, escape to infinity, and singularities in Celestial Mechanics.

We will view (1.0.1) as an equation for $q=\left(q_{1}^{\dagger}, \ldots, q_{N}^{\dagger}\right)^{\dagger} \in \mathbb{R}^{3 N}$. The equations of motion (1.0.1) are real analytic everywhere except where two or more of the particles occupy the same point in $\mathbb{R}^{3}$. More precisely, for $j \neq k$, let

$$
\begin{aligned}
\Delta_{j k} & =\left\{q \mid q_{j}=q_{k}\right\} \\
\Delta & =\bigcup_{1 \leq j<k \leq N} \Delta_{j k} .
\end{aligned}
$$

$\Delta$ is called the collision set; it is the union of $\frac{N}{2}(N-1)$ codimension-3 subspaces of $\mathbb{R}^{3 N}$. $U$ is real analytic on $\mathbb{R}^{3 N} \backslash \Delta$.

The standard existence and uniqueness theorems tell us that through a given starting position $q(0)$ and velocity $\dot{q}(0)$, there is a unique solution $q(t)$, defined for all $0 \leq t<\sigma$, for some maximal $\sigma$. It remains a challenge to determine the maximal interval of existence when $\sigma$ is finite; the more so with $\sigma=\infty$.

Definition. If $\sigma<\infty$, then $q(t)$ is said to experience a singularity at $\sigma$.

A familiar example in a much simpler setting is the one-dimensional problem: $\dot{x}=-\frac{1}{x}, x(0)=1$. Here $x=0$ is a singular point for the differential equation, and there is a solution $x=\sqrt{1-2 t}$, for which $x\left(\frac{1}{2}\right)=0$, and $\dot{x}\left(\frac{1}{2}\right)=-\infty$, so $\sigma=1 / 2$. We have a result of Painlevé from 1897 [80] that even if the singularity at $\sigma$ isn't a collision, $q$ must nevertheless approach the collision set:

FACT. [Painlevé] If $q(t)$ is singular at $\sigma$, then $q(t) \rightarrow \Delta$, as $t \rightarrow \sigma^{-}$.

Proof. First, if $q(t)$ is singular at $\sigma$, then $U \rightarrow \infty$ as $t \rightarrow \sigma^{-}$, since otherwise $U$ is bounded and every particle separation is bounded from below, so the right hand side of (1.0.1) is continuous at $t=\sigma$, and the existence theorem says that the solution continues beyond $\sigma$, contradicting the singularity. Since $U$ is unbounded, one of its terms must be, and the minimum of the mutual distances must tend to 0 as $t \rightarrow \sigma^{-}$, and thus $q(t)$ must approach $\Delta$.

Moreover, if $q(t)$ is singular at $\sigma$, then since $U \rightarrow \infty$ as $t \rightarrow \sigma^{-}$as in the proof above, by the conservation of energy, we must have that the kinetic energy $T=\sum_{i=1}^{N} m_{i}\left\|\dot{q}_{i}\right\|^{2}$ is also unbounded, so one of its terms must be, so some particle velocity becomes unbounded as $t \rightarrow \sigma^{-}$.

If $q(t)$ approaches a specific point $\hat{q} \in \Delta$, then the singularity is a collision, say $\hat{q}_{j}=\hat{q}_{k}$ for some $j \neq k$.

Definition. If $q$ experiences a singularity at $\sigma$, but $q(t)$ does not approach a specific point $\hat{q} \in \Delta$, then $q$ has a non-collision singularity.

The question of non-collision singularities has been studied for 120 years. Von Zeipel [112] showed in 1908 that

FACT. [Von Zeipel] A non-collision singularity can only occur if the system of particles becomes unbounded in finite time.

Having appeared in an obscure journal, von Zeipel's result went practically unnoticed until 1941, when Wintner [116] claimed the proof had gaps. In 1986, McGehee [73] filled in the details, showing that the initial argument had been correct.

Xia gave in 1992 the first example of a non-collision singularity for the $N$-body problem where $N \geq 5$ $[117]$.

Definition. $q$ is an escape trajectory if one or more $\left\|q_{i}\right\|$ tends to $\infty$ as $t \rightarrow \sigma^{-}$, where $\sigma \leq \infty$.

Our study is concerned with the problem of an expanding universe as defined below. 
Definition. The universe is expanding if the system of equations (1.0.1) possess solutions $q_{i}(t), i=$ $1, \ldots, N$ that exist on a semi-infinite interval $\left[t_{0}, \infty\right)$ with $\lim _{t \rightarrow \infty}\left\|q_{i}(t)-q_{j}(t)\right\|=\infty, i \neq j$.

Solutions to an expanding universe beg numerous questions, including: How large is the family of solutions to an expanding universe? Is it possible to associate with them a series representation that is absolutely and uniformly convergent on a semi-infinite interval? How fast is the convergence? Is it possible to obtain approximations as well as asymptotic approximations to any order of accuracy? This dissertation will provide some answers to these questions.

The existence question of solutions for all forward time has received much attention. In Birkhoff's text [12] page 291 on long-term behavior for $N>3$, "We shall entirely put to one side the question of collisions." I don't think this means he wasn't interested in that the existence of solutions (1.0.1) on a semi-infinite interval without singularities. Pollard's seminal 1967 paper [86], which was a major inspiration for the present work, includes a paragraph

"Two assumptions are made throughout. The first is a matter of convenience; the second is forced upon us by the total ignorance concerning the possible singularities which can occur. We assume first that the center of mass 0 is fixed. ... Secondly, we suppose that the coordinates of the particles in some rectangular coordinate system centered at 0 encounter no singularity for positive time $t . "$

Hence for his work, he proves properties of solutions that he assumes exist for all positive time. The present work addresses the second assumption of Pollard, which same assumption is made by many authors, as is summarized here.

Pollard [87] provides a detailed description of the Kepler problem; the only singularities are collisions, and a collision only happens in the linear problem. So there is an open set in the space of initial conditions whose solutions exist for all forward time. The same is of course true for the $N=2$ problem. It was shown by Painlevé [80] that the only singularities for the 3-body problem are collisions and by Saari [95] that $C S$ the set of initial conditions of (1.0.1) leading to collisions is of measure 0 and of Baire first category (a countable union of nowhere dense sets). An immediate consequence is the existence result for $N=3$ : The solution through almost all initial conditions exists for all positive time. Note that the complement of $C S$ is a countable intersection of open sets, so it need not be an open set, so this is not as strong as the result for $N=2$.

In 1976 Saari announced [96] for $N=4$ that Sing, the set of initial conditions leading to singularity, is of measure 0 and first category, and the proof was published in 1977 [97]. This is the same existence result as for $N=3$. It is not known whether $N C$ is empty for $N=4$.

There appears to be no comparable result for $N>4$. Saari has also shown in several papers in the early 70s, summarized in [95] for all $N$ that $C S$ is of measure 0 and first category. It is expected, but not known, that $N C$, the set of initial conditions leading to non-collision singularity, is also small in some sense, which would similarly imply existence for all positive time for most (in the same sense) initial conditions. Thanks to Xia [117] we know that $N C$ is not empty for $N \geq 5$. Saari and Xia have a 1996 paper [100] on singularities including a conjecture that the set $N C$ is the same as a certain set derived from $C S$. However, their conjecture would not imply that Sing is small.

To be sure, there are known solutions in the literature, chiefly central configurations, which Moeckel [76] notes in an accessible summary are the only explicitly known solutions. We offer by means of our construction a multi-parameter set of initial conditions through which solutions exist for all positive time. The Mingarelli's [75] have provided a study of bounded solutions that exist for all time.

The present work provides a construction of solutions and asymptotic approximations to any order for our positive energy solutions. Moreover, these solutions do not depend on regularizing after double collisions; we have found an open set of initial conditions through which singularity-free solutions escape to infinity. We also have a multi-parameter set of singularity-free solutions, in which all particles but one escape to infinity as $t \rightarrow \infty$.

Assuming solutions that exist for all time, there has been much asymptotic information published. The asymptotic behavior for the Kepler problem and the $N=2$ problem is well known [87], depending on the sign of the total energy $h$, bounded for $h<0$ and unbounded otherwise. In the unbounded case, the particle separation grows like a multiple of time, asymptotically. For collisions, Pollard's text [87] notes that near a collision in the 2-body problem, the particle separation shrinks as $(|t-\sigma|)^{2 / 3}$, so their relative velocity tends 
to infinity like $(|t-\sigma|)^{-1 / 3}$. Chazy $[\mathbf{1 7}]$ devoted a substantial amount of effort and space, providing a detailed analysis for the three types of motion for $N=3$ : hyperbolic $h>0$, parabolic $h=0$, and elliptic $h<0$. All three cases required a substantial amount of sophisticated transformations of the dependent and independent variables that are not easy to extend to the $N>3$ case. It is evident from Chazy and later analysis that for $N=3$, the elliptic case $h<0$ has far richer dynamics than $h \geq 0$, as the former involves oscillatory motion. It goes without saying that the complications are compounded for $N>3$. Noteworthy and remarkable is also the "Psi Series," an unconventional type of approximation and/or asymptotic expansion. An expansion and representation of solutions for $N=3$ and $h>0$ is provided utilizing a polynomial of the variables $1 / t$ and $\log t / t$.

In Chapter 5, we obtain a representation of solutions for the relatively simple case of $h>0$ and arbitrary $N>3$ and also obtain an asymptotic expansion for the solutions as $t \rightarrow \infty$ via integral equations. This work is also influenced by Chazy who used the leading terms $a_{i} t+b_{i} \log t+c_{i}$, and this work should be considered from the point of view of goals and techniques an extension of his work to $N>3$ using integral equations.

Let the maximum and minimum particle separations be

$$
R(t)=\max \left\|q_{i}-q_{j}\right\| \text { and } r(t)=\min _{i \neq j}\left\|q_{i}-q_{j}\right\| .
$$

Then, Chazy [17] on page 40 shows that for all $N$, assuming that solutions exist for all positive time and that the energy is positive, the limit

$$
L=\lim _{t \rightarrow \infty} \frac{r}{R}
$$

exists; the limit is obviously finite. Moreover, for $L \neq 0$ (which holds for all of our solutions), he shows that the potential energy $U \sim A / t$ for some $A>0$, and that the positions $q_{i}$ take the form (1.0.2), where each $\delta_{i}$ is a series in powers of $\frac{\log t}{t}$ and $\frac{1}{t}$. In a footnote, Chazy credits Bohlin [13] for the form of $q_{i}$ in the case of two and three bodies, and notes that Bohlin did not prove that the series converge. Chazy didn't prove convergence either, but Chapter 5 does, so we have confirmed Bohlin's form of the equations and proven that those solutions do exist for all time.

Chazy's result was refined and related to escape in a series of papers $[\mathbf{7 0 , 8 6 , 9 0 , 9 4 ]}$ starting in the late 60 s, wherein under the assumption that solutions exist for all forward time, Pollard, Saari, and collaborators gave additional conditions on solutions under which particles escape as $t \rightarrow \infty$, as well as growth estimates of the particles' position vectors and their mutual separation distances. These references include an analysis of the asymptotic behavior of solutions of equation (1.0.1) for an expanding universe. Common to all these articles characterizing such solutions is the assumption that the solution exists on $[0, \infty)$. Also common to all these studies is the use of the Sundman inequality and the Lagrange-Jacobi identity; in addition, Tauberian theorems play a vital role. In retrospect, it is remarkable how much asymptotic information they succeeded in obtaining given the tools they had to work with. It is noteworthy how far the theory of an expanding universe for $N>3$ was advanced based on a few scalar relations.

While we have in common with the references mentioned above the desire to study solutions of an expanding universe and obtain asymptotic relations for them as $t \rightarrow \infty$, several differences exist and are now being elaborated upon. First we notice the differences in the methods of construction and proof. Our technique is based on integral equations and the method of successive approximations for the vector solutions of (1.0.1) utilizing a vector formulation. The differences in techniques also explain one of the the differences in the nature of the results obtained. As seen from Theorem 29 and comparing with prior references on an expanding universe, rather than making the assumption that solutions free of singularities exist on a semi-infinite interval we prove this result while providing a construction of the vector solutions and their asymptotic expansions.

We construct a series solution representation on a semi-infinite interval that induces an asymptotic expansion for the solution to any order of accuracy. It turns out that the rate of convergence is very fast, which could render the series representation and the asymptotic representation a useful tool for numerical approximations. In particular, as pointed out by Diacu [26], the global series solutions of the 3-body problem as provided by Sundman [108] (with non-zero angular momentum) and the series solution provided by Wang [113] for the $N>3$ converge extremely slowly, so slowly in fact that the series provides no useful information [26]. It is also noteworthy that these series solutions do not identify the singularities; that is, one cannot determine from the series whether a given initial condition leads to a singularity. We also note the 1994 work of Saari and Diacu [99] on faster than At expansion. 


\subsection{Compactifications, Old and New}

This Section is dedicated to the review to the exposition and to the promotion of the study of solutions of differential systems and dynamical systems in the "neighborhood of infinity". This study promotes and utilizes a certain non-traditional compactification. We describe the theoretical background necessary to define neighborhoods and critical points at infinity of solutions of differential systems. We develop an explicit formula for the leading asymptotic term of diverging solutions to critical points at infinity. Applications to problems of completeness and incompleteness of dynamical systems are also brought to the fore. A simple bijection, $y=\left(1-x^{\dagger} x\right)^{-1} x, x, y \in \mathbb{R}^{k}$, with $\|x\|<1$, that has a rich geometrical interpretation, plays a major role in our study.

In Chapter 2, we will describe some results obtained on compactifying some polynomial systems in $\mathbb{R}^{k}$, including the celebrated Lorenz system, and in Chapter 3 apply this compactification to Celestial Mechanics, where $k=6 N$.

The projection of the real line onto a circle with a point removed is a form of compactification that was known to Greek mathematicians more than two thousand years ago. In 1881 Poincaré [85], studied limit cycles "at infinity" of two-dimensional polynomial differential equations via compactification. Although the paper contained errors that were addressed more than a hundred years later by Roeder [92], the original ideas had lasting impact. An early study of differential equations via compactification was carried out by Bendixson [10]; see Andronov et al [3], p. 216. Bendixson used the stereographic projection that does not account for all directions at infinity. See e.g. Ahlfors [2] and Hille [52], for versions of the stereographic projection. The Poincare compactification is adopted in various textbooks on differential equations $[\mathbf{6 0}, \mathbf{6 6}$, 83, 101]. It is widely used to study critical points at infinity; for example, the studies of Chicone and Sotomayor [18], Cima and Llibre [19], Schlomiuk and Vulpe [102], and their references. It is noteworthy that the stereographic projection is obtained by Gingold and Gingold [39] as a degenerate limit of a family of compactifications that account for all directions at infinity. However, that compactification is akin to the Poincaré compactification [85] and possesses radicals that prevent it being a tool for rational approximations. The parabolic compactification used here was proposed by Gingold [38] in 2004, and first made use of in ODEs by Elias and Gingold [35] in 2006. Moreover, they extended and generalized this compactification to a family of radial compactifications. Compactification is an excellent means to obtain global phase portraits of vector fields of dynamical systems that include the neighborhood of infinity. The work of Cima and Llibre [19] is a welcome global analysis and supplement to that of Chen and Liang [15]. Numerous applications of the Poincare compactification were applied to polynomial systems $[\mathbf{3}, \mathbf{1 8}, \mathbf{1 9}, \mathbf{6 0}, \mathbf{8 3}, \mathbf{8 5}]$. See e.g. Willard [115] for topics of compactification in general topology.

Our unconventional compactification $y=\frac{x}{1-x^{\dagger} x}$ has advantages over the stereographic projection utilized by Bendixson [10] because ours distinguishes directions at infinity. Our compactification has also an advantage over the widely used Poincaré compactification (see e.g. [3, 60] for an exposition), because it transforms a polynomial differential system into a rational system, whereas the Poincaré compactification introduces radicals. Compactification offers a natural and systematic approach to study solutions of $\dot{y}=\frac{d y}{d t}$ for $\|y(t)\|$ large or unbounded. In addition, compactification facilitates the manipulation of unbounded quantities and can provide us with a priori bounds, which are not obvious otherwise [38].

The usual meaning that a sequence of points $z(n) \in \mathbb{R}^{k}, \quad n=1,2, \ldots$ diverges to infinity in $\mathbb{R}^{k}$, that $\lim _{n \rightarrow \infty}\|z(n)\|=\infty$, loses any sense of the asymptotic direction of $z$. The definition is compatible with a common one in stereographic projection in complex analysis that is associated with the extended complex plane. Just one geometrical point corresponding to the symbol $\infty$ augments $\mathbb{R}^{2}$; see e.g. [2, 52]. However, This definition ignores the distinction between the different directions at infinity.

Definition. We say that the unbounded sequence $z(n), n=0,1,2, \ldots$ diverges in the direction $p$ to infinity or diverges to $\infty p,\|p\|=1$, if

$$
\lim _{n \rightarrow \infty}\|z(n)\|=\infty \text { and } \lim _{n \rightarrow \infty} \frac{z(n)}{\|z(n)\|}=p .
$$

Denote by $\left(t_{\min }, t_{\max }\right)$, where $-\infty \leq t_{\min }<0<t_{\max } \leq \infty$, the maximal interval of existence of a solution of a differential system

$$
\dot{y}=\frac{d y}{d t}=f(y), y(0)=y_{0}
$$


One reason that $t_{\min }$ or $t_{\max }$ could be finite is that the solution or its derivative become unbounded near an endpoint. The continuous analog of diverging in the direction $p$ to infinity in $\mathbb{R}^{k} p$ is given by

Definition. We say that the vector function $y(t) \in C\left(t_{\min }, t_{\max }\right)$ diverges in the direction $p$ to infinity, or diverges to $\infty p$, denoted

$$
\lim _{t \rightarrow t_{\min }^{+}} y(t)=\infty p \quad \text { or } \quad \lim _{t \rightarrow t_{\max }^{-}} y(t)=\infty p
$$

if

$$
\lim _{t \rightarrow t_{\max }^{-}}\|y(t)\|=\infty \quad \text { and } \quad \lim _{t \rightarrow t_{\max }^{-}} \frac{y(t)}{\|y(t)\|}=p
$$

or

$$
\lim _{t \rightarrow t_{\min }^{+}}\|y(t)\|=\infty \quad \text { and } \quad \lim _{t \rightarrow t_{\min }^{+}} \frac{y(t)}{\|y(t)\|}=p .
$$

Analogous to Gingold and Gingold [39], we define an Ultra Extended $\mathbb{R}^{k}$ and produce an induced metric.

Definition. the Ultra Extended $\mathbb{R}^{k}, U E \mathbb{R}^{k}$ is the union of $\mathbb{R}^{k}$ and a certain ideal set $I D$, where

$$
I D:=\{\infty p \mid\|p\|=1\}, U E \mathbb{R}^{k}:=\mathbb{R}^{k} \cup I D .
$$

As seen in the sequel there is good reason to introduce nonlinear transformations that will allow us to reduce the investigation of differential systems with unbounded solutions to the investigation of differential systems with a priori bounded solutions.

In preparation to transforming the equation $\dot{y}=f(y)$ we need a diffeomorphism that will facilitate computations and will take the space $\mathbb{U E} \mathbb{R}^{k}$ into a bounded set. We sketch the main ideas. For more details see Gingold [38]. We project the point $y=\left(y_{1}, \ldots, y_{k}, 0\right) \in \mathbb{R}^{k+1}$ through the point $(0, \ldots, 0,1)$ on the surface (1.2.6)

$$
x_{k+1}=\left(x_{1}^{2}+\ldots+x_{k}^{2}\right)^{1 / 2}
$$

and single out $Z=\left(x_{1}, x_{2}, \ldots, x_{k}, x_{k+1}\right)$ as one of the two points of intersection of the parabolic surface (1.2.6) and the straight line connecting $\left(y_{1}, \ldots, y_{k}, 0\right)$ and $(0, \ldots, 0,1)$. The determination of $Z$ will be done by the determination of a certain branch of a multi-valued function as given below. Then, all the points $y=\left(y_{1}, \ldots, y_{k}, 0\right)$ map onto a parabolic bowl with coordinate $x_{k+1}=\left(x_{1}^{2}+\ldots+x_{k}^{2}\right)^{1 / 2}<1$, $\left(x_{1}, \ldots, x_{k}, x_{k+1}\right) \in \mathbb{R}^{k+1}$ and all the points $\infty p,\|p\|=1$, map onto the "circle" with $x_{k+1}=\left(x_{1}^{2}+\ldots+\right.$ $\left.x_{k}^{2}\right)^{1 / 2}=1$. Denote by $U$ the open unit ball and by $\partial U$ its boundary, the unit sphere.

$$
U:=\left\{x \in \mathbb{R}^{k} \mid\|x\|<1\right\}, \partial U:=\left\{x \in \mathbb{R}^{k} \mid\|x\|=1\right\} .
$$

Denote the magnitudes $r=\|y\|, R=\|x\|$. Then the transformation

$$
y=\frac{x}{1-R^{2}}, r=\frac{R}{1-R^{2}}
$$

illustrated in Figure 1.2 is shown in [38] to be a bijection from $\mathbb{R}^{k}$ onto $U$. It extends to a bijection from the ideal set $I D$ onto $\partial U$. The inverse of $y=\frac{x}{1-R^{2}}$ in $R<1$ is defined by the branch

$$
x=\frac{2 y}{1+\sqrt{1+4 r^{2}}}, R=\frac{2 r}{1+\sqrt{1+4 r^{2}}} .
$$

The compactification (1.2.8) induces a metric in $U E \mathbb{R}^{k}$ in a natural manner. We consider two points $y, \hat{y} \in U E \mathbb{R}^{k}$. Denote their images under the above bijection by $x, \hat{x}$ respectively. Define a positive definite function $M(y, \hat{y})$ by the Euclidean distance between the compactified points

$$
M(y, \hat{y}):=\|x-\hat{x}\| .
$$




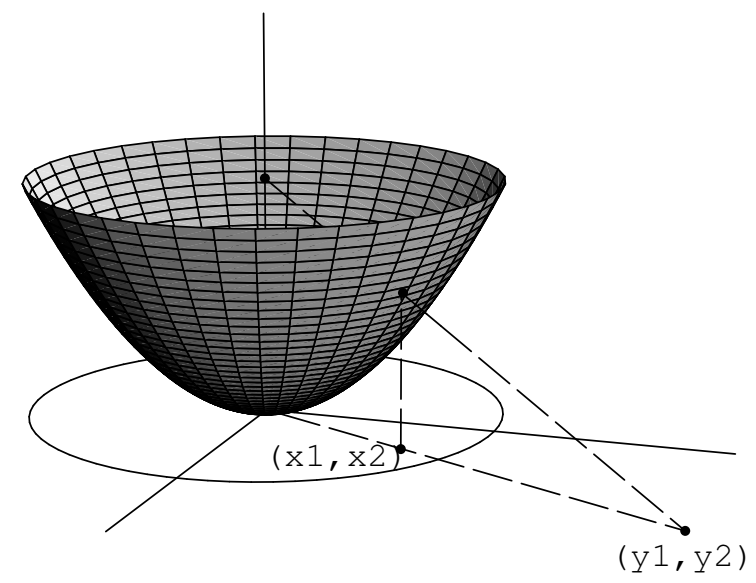

FiguRE 1.2.1. Compactification of $\mathbb{R}^{2}$

If $y \notin \mathbb{R}^{k}$, then $y=\infty p$; similarly, either $\hat{y} \in \mathbb{R}^{k}$ or $\hat{y}=\infty \hat{p}$. Let $\hat{r}=\|\hat{y}\|, \theta=\frac{2}{1+\sqrt{1+4 r^{2}}}, \hat{\theta}=\frac{2}{1+\sqrt{1+4 \hat{r}^{2}}}$. It is shown by Gingold [38] that $U E \mathbb{R}^{k}$ is a complete metric space. For the different cases, the chordal metric is given by the following for $y, \hat{y} \in \mathbb{R}^{k}$

$$
\begin{gathered}
M(y, \hat{y})=\sqrt{\theta \widehat{\theta}\|y-\hat{y}\|^{2}-\frac{(\theta-\hat{\theta})^{2}(1-\theta \hat{\theta})}{\theta \hat{\theta}}} \\
M(\infty p, \hat{y})=\sqrt{1-2 \hat{\theta} p^{\dagger} \hat{y}+\hat{\theta}^{2} \hat{r}^{2}+\left(1-\hat{\theta}^{2} \hat{r}^{2}\right)^{2}} \\
M(\infty p, \infty \hat{p})=\sqrt{2\left(1-p^{\dagger} \hat{p}\right)} .
\end{gathered}
$$

This allows divergence (in a direction) of solutions of dynamical systems to be dealt with as convergence to the boundary of the compactified system. It is easy to see that if $y(t)$ diverges to $\infty p$, then $\theta r \rightarrow 1$ and $\theta y \rightarrow p$, so $M(y, \infty p) \rightarrow 0$.

We turn now to the compactification of a polynomial differential system. Let $y$ be a column vector in $\mathbb{R}^{k}$. Let $y^{\dagger}=\left(y_{1}, y_{2}, \ldots, y_{k}\right)$ denote a row vector that is the transpose of $y$. In particular let $\hat{0}^{\dagger}=(0, \ldots, 0)$ be the transpose of the zero vector. Let $f^{\dagger}(y):=\left(f(y)_{1}, f(y)_{2}, \ldots, f(y)_{k}\right)$ be a vector field in $\mathbb{R}^{k}$ where $f(y)_{j}, j=1,2, \ldots, k$ are scalar polynomial functions. We say that $\frac{d y}{d t}=f(y)$ is a polynomial differential system of degree $L$ if the vector function $f(y)$ is given by

$$
f(y)=f_{0}(y)+f_{1}(y)+\ldots f_{L-1}(y)+f_{L}(y),
$$

where $f_{j}(y), j=0,1,2, \ldots, L$ are homogeneous polynomial column vectors of degree $j$ and $f_{L}(y) \neq \overrightarrow{0}$ for some $y \in \mathbb{R}^{k}$. [Note the difference between $f(y)_{j}$ and $f_{j}(y)$ ]. Now, for $f$ a polynomial of degree $L$, let

$$
\begin{gathered}
\tilde{f}(x):=\left(1-R^{2}\right)^{L} f\left(\frac{x}{1-R^{2}}\right) \\
=\left(1-R^{2}\right)^{L} f_{0}+\left(1-R^{2}\right)^{L-1} f_{1}(x)+\ldots+\left(1-R^{2}\right)^{2} f_{L-2}(x)+\left(1-R^{2}\right) f_{L-1}(x)+f_{L}(x) .
\end{gathered}
$$

Then the compactification (1.2.8) takes the differential system (1.2.2) into the differential system

$$
\frac{d x}{d t}=\frac{\left[\left(1+R^{2}\right) I-2 x x^{\dagger}\right] \tilde{f}(x)}{\left(1+R^{2}\right)\left(1-R^{2}\right)^{L-1}},
$$


from which we have

$$
\frac{d\left(1-R^{2}\right)}{d t}=2 x^{\dagger} \dot{x}=-2 x^{\dagger} \frac{\left[\left(1+R^{2}\right) I-2 x x^{\dagger}\right] \tilde{f}(x)}{\left(1+R^{2}\right)\left(1-R^{2}\right)^{L-1}}=-\frac{2 x^{\dagger} \tilde{f}(x)}{\left(1+R^{2}\right)\left(1-R^{2}\right)^{L-2}} .
$$

Definition. A point $y_{0}$ is a critical point of the differential equation $\dot{y}=f(y)$, if $f\left(y_{0}\right)=0$.

Note that a critical point can only be reached along a trajectory as time goes to infinity. Our system has no critical points, because if every $\ddot{q}_{i}=0$ at a point $q$, then every $\frac{\partial U}{\partial q_{i}}=0$, and trivially, $\sum_{i} q_{i} \frac{\partial U}{\partial q_{i}}=0$, but since $U$ is homogeneous of degree -1 , the sum is equal to $-U$ by a theorem of Euler. So $U(q)=0$, which is impossible. The following important result is from [35]

LEMma 1. The transformation (1.2.8) maps critical points of (1.2.2) in $\mathbb{R}^{k}$ into critical points of (1.2.13) in $U$, and vice versa.

Proof. If $y$ is a critical point of (1.2.2); i.e., $f(y)=0$, then the right-hand side of (1.2.13) obviously vanishes at the corresponding $x$. Conversely, suppose that the right-hand side of (1.2.13) vanishes for some $x \in U$; then we must have

$$
\tilde{f}(x)-\frac{2}{1+R^{2}} x x^{\dagger} \tilde{f}(x)=0 .
$$

Applying $x^{\dagger}$ to this equation we get

$$
x^{\dagger} \tilde{f}(x)\left(1-\frac{2 R^{2}}{1+R^{2}}\right)=x^{\dagger} \tilde{f}(x) \frac{1-R^{2}}{1+R^{2}}=0 .
$$

The fraction is positive, since $x$ is an interior point, so $x^{\dagger} \tilde{f}(x)$ must be 0 , and by (1.2.15), $\tilde{f}(x)=0$, so $f(y)=\left(1-R^{2}\right)^{-L} \tilde{f}(x)=0$, and $y$ is a critical point.

\subsection{The Flow on the Boundary and at $\infty$}

This Section is about the compactified flow on the boundary and its critical points, as well as insights into the system for large $\|y\|$ to be gained from trajectories in and near the boundary. The flow in the boundary is in many cases comparatively simple to analyze. As we will see in Chapter 2, for the Lorenz system and some other systems, the boundary sphere is an invariant set. We show in Chapter 2 that the boundary repels, and is in fact the dual repeller to the compactification of the well-known Lorenz attractor.

For polynomial systems, the right side of (1.2.13) is singular on the boundary, preventing us from extending the compactified system to the boundary. We rescale time as developed in [35] and [38] by considering $x$ and $t$ as functions of a new independent variable $\tau \in(-\infty, \infty)$, where

$$
\begin{gathered}
\frac{d t}{d \tau}=\left(1+R^{2}\right)\left(1-R^{2}\right)^{L-1}, \quad t(0)=t_{0} \in\left(t_{\min }, t_{\max }\right) \\
\frac{d x}{d \tau}=\left(1+R^{2}\right) \tilde{f}(x)-2 x^{\dagger} \tilde{f}(x) x, \quad x(0)=x_{0} .
\end{gathered}
$$

As before, we have

$$
\frac{d\left(1-R^{2}\right)}{d \tau}=-2 x^{\dagger}\left[\left(1+R^{2}\right) \tilde{f}(x)-2 x^{\dagger} \tilde{f}(x) x\right]=-2\left(1-R^{2}\right) x^{\dagger} \tilde{f}(x), \quad 1-R(0)^{2}=1-x_{0}^{\dagger} x_{0} .
$$

The rescaled system (1.3.2) on the open unit ball can be continuously extended to the boundary; we view (1.3.2) as being defined on the closed unit ball. If $R(0)=\left\|x_{0}\right\|=1$, the right hand side of (1.3.3) vanishes, so $x(\tau)$ stays in the boundary for all time $\tau$, which means the boundary is invariant under (1.3.2). Moreover, since $1-R^{2}=0$ is a critical point of (1.3.3), it can only be approached as $\tau \rightarrow \pm \infty$. For any $x_{0}$ in the closed unit ball, the initial value problems (1.3.2),(1.3.1),(1.3.3) possess unique solutions on $-\infty<\tau<\infty$. The approach of a trajectory $x(\tau)$ to the boundary is the compactification of a trajectory $y$ diverging to infinity, so we have shown

Proposition 2. If a trajectory $y(t)$ diverges to infinity in the direction $p$, then $p$ is a critical point of (1.3.2) in the boundary. 
Since $\frac{d t}{d \tau}>0,(1.3 .1)$ generates a one-to-one mapping between the variable $\tau$ on $-\infty<\tau<\infty$ and the variable $t$ on $\left(t_{\min }, t_{\max }\right)$. We have then

$$
t-t_{0}=\int_{0}^{\tau}\left(1+R^{2}\right)\left(1-R^{2}\right)^{L-1} d \tau
$$

If it is possible to estimate the rate of decay of $1-R^{2}$, the following can be used to estimate $t_{\text {min }}$ and $t_{\text {max }}$

$$
t_{\max }-t_{0}=\int_{0}^{\infty}\left(1+R^{2}\right)\left(1-R^{2}\right)^{L-1} d \tau, \quad t_{0}-t_{\min }=\int_{-\infty}^{0}\left(1+R^{2}\right)\left(1-R^{2}\right)^{L-1} d \tau ;
$$

in particular, if both integrals diverge, the solution $y(t)$ exists for all time $t$. More generally, we have the qualitative information that the larger $L$ is, the smaller $\left(1-R^{2}\right)^{L-1}$ could become and therefore the smaller $t_{\max }-t_{0}$ and $t_{0}-t_{\min }$ may become.

Next we give a rigorous footing to the notion of a critical point $\infty p$ of a dynamical system using the compactification.

Definition. We say that $\infty p$ is a critical (equilibrium) point or that $p$ is a critical direction of (1.2.2) at infinity if $p$ is a critical point of (1.3.2).

If $p$ is a critical point at infinity, then from (1.3.2), we must have

$$
\tilde{f}(p)=p^{\dagger} \tilde{f}(p) p
$$

or, since for any point $p$ on the boundary, $\tilde{f}(p)=f_{L}(p)$

$$
f_{L}(p)=p^{\dagger} f_{L}(p) p=p p^{\dagger} f_{L}(p) .
$$

If in addition we have $p^{\dagger} f_{L}(p) \neq 0$ then

$$
p=\frac{f_{L}(p)}{p^{\dagger} f_{L}(p)}
$$

which implies, on taking magnitudes, that $\left\|f_{L}(p)\right\|=\left|p^{\dagger} f_{L}(p)\right|$. So

$$
p= \pm \frac{f_{L}(p)}{\left\|f_{L}(p)\right\|}
$$

and we call $\infty p$ a generic critical point at infinity, and we call $p$ a generic critical direction at infinity. The more interesting critical points at infinity are the points $\infty p$ that are limits of $y$ as $t$ tends to $t_{\text {min }}$ or $t_{\text {max }}$.

The set of initial points $y_{0} \in \mathbb{R}^{k}$ such that the unbounded solutions of the initial value problem $\dot{y}=$ $f(y), y\left(t_{0}\right)=y_{0}$, satisfies (1.2.3) or (1.2.4), is called the basin of divergence of $\infty p$ or the basin of divergence in the $p$ direction. Notice that by this definition at least one value of $x_{0} \neq p$ must be included in the basin of divergence of $p$.

REMARK 3. The set of critical points at infinity of a compactified and parametrized equation are not well defined without a certain normalization that needs to be introduced or is implicitly assumed. In the above treatment we "naturally" but arbitrarily defined a parametrization (1.3.1). This determination causes the remaining equations (1.3.2) and (1.3.3) to be uniquely determined. However, one may introduce spurious critical points as follows. Consider

$$
\frac{d x}{d s}=g(x)\left[\left(1+R^{2}\right) \tilde{f}(x)-2 x^{\dagger} \tilde{f}(x) x\right]
$$

with

$$
g(x)=\prod_{j=1}^{k}\left(x_{j}-x_{j, 0}\right)^{2 m_{j}}, \quad m_{j} \in \mathbb{N}, \quad \sum_{j=1}^{k} x_{j, 0}^{2}=1 .
$$

Then the equation pertaining to $t$ would be

$$
\frac{d t}{d s}=g(x)\left(1+R^{2}\right)\left(1-R^{2}\right)^{L-1},
$$


and then the point $\left(x_{1,0}, x_{2,0}, \ldots, x_{k, 0}\right)$ can be made to be a spurious critical point at infinity. It is noteworthy that the case $k=1$ differs from the case $k>1$. For $k=1$, (1.3.2) becomes

$$
\frac{d x}{d \tau}=\left[\left(1+R^{2}\right) I-2 x x^{\dagger}\right] \tilde{f}(x)=\left(1-R^{2}\right) \tilde{f}(x) .
$$

Then $x=( \pm 1)$ are the only two critical points of (1.3.10) so that $\infty( \pm 1)$ are the only two critical points at infinity of a scalar polynomial differential equation. However, it seems desirable to choose for $k=1 \mathrm{a}$ different parametrization with

$$
g(x)=\left(1-R^{2}\right)^{-1}, \quad \frac{d x}{d s}=2 \tilde{f}(x), \quad \frac{d t}{d s}=\left(1+R^{2}\right)\left(1-R^{2}\right)^{L-2} .
$$

This will eliminate the common factor of the right hand sides of

$$
\frac{d x}{d \tau}=2 \tilde{f}(x)\left(1-R^{2}\right) \quad \text { and } \quad \frac{d t}{d \tau}=\left(1+R^{2}\right)\left(1-R^{2}\right)^{L-1} .
$$

Then, if $L>0, x=( \pm 1)$ will not be critical points of $\frac{d x}{d s}=2 \tilde{f}(x)$.

The compactification shows that every polynomial differential system possess at least one critical point in the Ultra Extended $\mathbb{R}^{k}$. $U E \mathbb{R}^{k}$.

Proposition 4. A polynomial differential system with $L>0$ possesses at least one critical point in the

Proof. If $f(y)=\hat{0}$ for some $y \in \mathbb{R}^{k}$ then we are done. Assume now without loss of generality that there does not exist $y \in \mathbb{R}^{k}$ such that $f(y)=\hat{0}$. By Lemma $1, x(\tau)$ has no critical points in the interior, and in particular by (1.3.2), for every point $x$ in the interior, $\tilde{f}(x) \neq \hat{0}$. If there is a point $x$ on the boundary with $\tilde{f}(x)=0$, then we are done. If not, then the mapping

$$
w(x):=\frac{\tilde{f}(x)}{\|\tilde{f}(x)\|}
$$

is a continuous mapping from $\bar{U}$ into $\partial U \subset \bar{U}$. By Brower's fixed point theorem there exists $x \in \partial U$ such that

or

$$
x=w(x)=\frac{\tilde{f}(x)}{\|\tilde{f}(x)\|},
$$

$$
\tilde{f}(x)=\|\tilde{f}(x)\| x
$$

which we substitute into

$$
\frac{d x}{d \tau}=2\left[I-x x^{\dagger}\right] \tilde{f}(x),
$$

which is what (1.3.2) reduces to on the boundary, to obtain

$$
\frac{d x}{d \tau}=2\left[I-x x^{\dagger}\right]\|\tilde{f}(x)\| x=2\|\tilde{f}(x)\|\left[x-R^{2} x\right]=\hat{0},
$$

since $R^{2}=1$, and the result follows.

In Chapter 2, we will consider the flow of (1.3.2) restricted to the boundary for its insight into behavior of $y$ for large $\|y\|$. Recall that the system on the boundary is much simplified, in particular because on the boundary, $\tilde{f}(x)=f_{L}(x)$; that is, the flow on the boundary depends only on the highest order terms in $f$. The Jacobian at a critical point is a familiar tool for analysis of trajectories near a critical point, and this is available here, as well. The following is proven in [35]

Proposition 5. The Jacobian of (1.3.2) at a critical point $x \in \partial U$ is determined by the components $f_{L}(x)$ and $f_{L-1}(x)$ and is given by

$$
J_{i j}=\frac{\partial f_{L, i}}{\partial x_{j}}-x_{i} x^{\dagger} \frac{\partial f_{L}}{\partial x_{j}}-x^{\dagger} f_{L} \delta_{i j}-2\left(f_{L-1, i}-x^{\dagger} f_{L-1} x_{i}\right) x_{j} .
$$


As an example of the usefulness of these approaches, the following result proven in [35] gives the rate of blow up of a solution $y$ with a critical point in the direction $p$

Proposition 6. Suppose the eigenvalues $\lambda_{1}, \ldots \lambda_{k}$ of the Jacobian (1.3.14) have negative real parts. Then there is a k-parameter family of solutions of (1.2.2), with $f(y)$ a polynomial of degree L, that each tend to infinity in the direction $p$ so that

$$
\|y\| \sim c\left(t_{\max }-t\right)^{-1 /(L-1)} \text { as } t \rightarrow t_{\max } .
$$

In particular, $y(t)$ is not defined for all time, so (1.2.2) is not complete.

For the Celestial Mechanics problem, the boundary is not an invariant set; nevertheless, critical points and trajectories in the boundary will be shown in Chapter 3 to attract near-by trajectories; this corresponds after uncompactifying to trajectories escaping to infinity. The flow on the boundary is very simple, and trajectories can be given in closed form. The critical point set in $U=\mathbb{B}^{6 N}$ is given by

$$
C P=\left\{x \mid \sum_{j=1}^{N} x_{j}^{\dagger} x_{j}=1, x_{N+j}=0\right\},
$$

which is the compactification of the subset

$$
y_{i}=\infty a_{i}, y_{N+i}=a_{i}
$$

of the ultra extended $\mathbb{R}^{6 N}$, where the $a_{i}$ are constant 3 -vectors.

\subsection{Notation Glossary and Key Definitions}

For any positive integer $m, O_{m}$ will denote the $m$ by $m$ matrix with all entries being zero, and $I_{m}$ is the $m$ by $m$ identity matrix.

$0_{m}$ is the column $m$-vector with all entries zero. We can specify a diagonal matrix by listing its diagonal elements:

$$
\operatorname{diag}\left\{a_{1}, a_{2}, \ldots, a_{n}\right\}=\left(\begin{array}{ccccc}
a_{1} & 0 & 0 & \ldots & 0 \\
0 & a_{2} & 0 & \ldots & 0 \\
0 & 0 & \ddots & 0 & 0 \\
\vdots & \vdots & 0 & \ddots & 0 \\
0 & 0 & 0 & 0 & a_{n}
\end{array}\right) .
$$

We will often employ a construct known as a "vector of vectors"; we have defined the $3 N$-vector $q$, which is composed of the $N$ three-vectors $q_{1}, q_{2}, \ldots, q_{N}$, or more formally $q=\left(q_{1}^{\dagger}, q_{2}^{\dagger}, \ldots, q_{N}^{\dagger}\right)^{\dagger}$. So for example, the square of the magnitude of $q$, which is the sum of the squares of the magnitudes of the $q_{i}$, could be expressed as

$$
\|q\|^{2}=q^{\dagger} q=\sum_{i=1}^{N} q_{i}^{\dagger} q_{i}
$$

Because we often combine position and velocity into a state vector, we will sometimes use $6 \mathrm{~N}$-vectors and matrices considered as composed of $3 N$-blocks. An illustrative example is given below in (4.1.13):

$$
\dot{\Delta}=J_{0} \Delta+F=\left(\begin{array}{cc}
-\frac{1}{t} I_{3 N} & I_{3 N}-x^{*} x^{* \dagger} \\
-\frac{24 A_{1}^{2}}{t^{2}} G^{*} x^{* \dagger} & -\frac{1}{t}\left[I_{3 N}+x^{*} x^{* \dagger}\right]
\end{array}\right) \Delta-8 A_{1}^{3}\left(\begin{array}{c}
\frac{1}{t^{4}} x^{*} x^{* \dagger} G^{*} \\
\frac{1}{t^{3}} G^{*}
\end{array}\right) .
$$

$\Delta$ is a $6 N$-vector (like $q$ ), and the matrix $J_{0}$ is $6 N$ by $6 N$, defined in terms of four $3 N$ by $3 N$ blocks. Since $x^{*}$ and $G^{*}$ are $3 N$-vectors, $x^{*} x^{* \dagger}$ and $G^{*} x^{* \dagger}$ are $3 N$ by $3 N$ matrices, and this information defines $J_{0}$ completely. Similarly, $F$ is a $6 N$-vector composed of the two $3 N$-vectors indicated.

We shall have occasion to use a multi-index, which is very neatly summarized in John's PDE text [59], wherein he credits Laurent Schwartz for the notation:

Definition. A multi-index $\alpha$ is a vector of non-negative integers, $\alpha=\left(\alpha_{1}, \ldots, \alpha_{n}\right), a_{i} \in \mathbb{N}$. 
Given a multi-index $\alpha$, we define the scalars

$$
|\alpha|=\alpha_{1}+\cdots+\alpha_{n}, \quad \alpha !=\alpha_{1} ! \ldots \alpha_{n} !
$$

and for a vector $x \in \mathbb{R}^{n}$, the monomial $x^{\alpha}=x_{1}^{\alpha_{1}} \ldots x_{n}^{\alpha_{n}}$. We can denote a quantity depending on $n$ nonnegative integers $\alpha_{1}, \ldots, \alpha_{n}: C_{\alpha}=C_{\alpha_{1}, \ldots, \alpha_{n}}$. Each $C_{\alpha}$ may be numbers or vectors, depending on the context. This enables us to express the general $m$-th degree polynomial in $x_{1}, \ldots x_{n}$ as $P(x)=\sum_{|\alpha| \leq m} C_{\alpha} x^{\alpha}$.

Using the symbol $D_{k}=\partial / \partial x_{k}$, we can express the gradient operator $D=\left(D_{1}, \ldots, D_{n}\right)$, so that the gradient of a function $u$ is given by

$$
D u=\left(D_{1} u, \ldots, D_{n} u\right),
$$

and the general partial derivative operator is given by

$$
D^{\alpha}=D_{1}^{\alpha_{1}}, \ldots, D_{n}^{\alpha_{n}}=\frac{\partial^{|\alpha|}}{\partial x_{1}^{\alpha_{1}} \ldots \partial x_{n}^{\alpha_{n}}} .
$$

As an example of the usefulness of this notation, we can express the Taylor polynomial of degree $m$ for a function $f(x) \in C^{m}$ as

$$
\sum_{|\alpha| \leq m} \frac{D^{\alpha} f(0)}{\alpha !} x^{\alpha} .
$$

Definition. We say that a function $f(t)$ is real analytic or simply analytic at a point $t_{0}$ if for points $x$ with $\left\|t-t_{0}\right\|$ sufficiently small, the Taylor series for $f$ converges to $f(t)$.

$$
f(t)=\sum_{m=0}^{\infty} \frac{d^{m} f\left(t_{0}\right)}{d t^{m}}\left(t-t_{0}\right)^{m}
$$

We will be interested in convergent series expansions in $1 / t$ for large $t$. Let $z=1 / t$; then a function $g(t)$ is analytic at $\infty$ if $f(z)=g(1 / z)$ is analytic at $z=0$. This means in particular that for sufficiently large $t$, $g(t)$ is equal to its convergent series

$$
g(t)=\sum_{m=0}^{\infty} \frac{c_{m}}{t^{m}}
$$

We will follow the conventional usage from asymptotic analysis as given by Olver [79]. Starting with a known function $\phi(t)$, we describe the behavior as $t \rightarrow \infty$ of a function $f(t)$ as follows:

Definition. The function $f$ is said to be asymptotic to $\phi, f(t) \sim \phi(t)$, as $t \rightarrow \infty$, or $\phi$ is an asymptotic approximation of $f$ if $f(t) / \phi(t) \rightarrow 1$. When there is no ambiguity, we will use the shorthand $f \sim \phi$.

The function $f$ is of order less than $\phi, f(t)=o(\phi(t))$, as $t \rightarrow \infty$, or $f$ is little o of $\phi$ if $f(t) / \phi(t) \rightarrow 0$. When there is no ambiguity, we will use the shorthand $f=o(\phi)$.

The function $f$ is of order no more than $\phi, f(t)=O(\phi(t))$, as $t \rightarrow \infty$, or $f$ is big $O$ of $\phi$ if $f(t) / \phi(t)$ is bounded. When there is no ambiguity, we will use the shorthand $f=O(\phi)$.

For example,

$$
\cos t=O(1), \log t=o(t), t^{2}+t \sim t^{2} .
$$

Highly desirable in applications are derivations with the symbol $\sim$ of asymptotic equivalence that provide much more accurate information than the symbols $o$ and $O$. Note in particular that the identically 0 function is both $o\left(t^{n}\right)$ and $O\left(t^{n}\right)$, as $t \rightarrow \infty$, for all real $n$, so $o$ and $O$ provide less information than $\sim$. A comparison between the formulas of our Theorem 29 and the formulas cited in Section 5.4 from Saari [94] and Marchal and Saari $[\mathbf{7 0}]$ show the differences. However, we will have occasion to use the following equivalence relation

Definition. Two positive continuous functions $f$ and $g$ are said to be of the same order, $f \approx g$ if after some time there exist constants $A$ and $B$ such that $A g(t) \leq f(t) \leq B g(t)$. This is the same as saying both $f=O(g)$ and $g=O(f)$, as $t \rightarrow \infty$.

For example, if $f$ and $g$ are polynomials of the same degree, then $f \approx g$. 
Definition. The open unit ball $\mathbb{B}^{k} \subset \mathbb{R}^{k}$ is $\{x \mid\|x\|<1\}$ and its boundary sphere $\mathbb{S}^{k-1}$ is $\{x \mid\|x\|=1\}$. For our purposes starting in Chapter $3, k$ will be $6 N, x$ is a vector of vectors as above, and we would express the unit ball as

$$
\mathbb{B}^{6 N}=\left\{x \mid \sum_{j=1}^{N}\left(x_{j}^{\dagger} x_{j}+x_{N+j}^{\dagger} x_{N+j}\right)<1\right\} .
$$

Note also that we follow Pollard [86] in using $\log t$ to refer to the natural logarithm of $t$, rather than the usual convention, $\ln t$.

We will be dealing with differential equations in the unit ball, some of whose coefficients are given in powers of $1 / t$. Since we are interested in long-term behavior, we will study these differential equations near $t=\infty$. This combination compels us to consider singularities of differential equations at $\infty$. There are two legendary sources that use different names for the same characterization: regular or irregular singularities (Wasow [114]) vs singularities of the first or second kind (Coddington \& Levinson [20]).

Definition. If the matrix $A(t)$ has a singularity at $t_{0}$, then $t_{0}$ is called a singular point for the system

$$
\dot{y}=A(t) y \text {. }
$$

Points that are not singular are called ordinary. If $\mathrm{A}$ has a pole at $t_{0}$, but is analytic in a punctured disc $0<\left|t-t_{0}\right|<a, a>0$, then $A$ may be expressed as

$$
A(t)=\left(t-t_{0}\right)^{-\mu-1} \tilde{A}(t),
$$

where $\mu \geq 0$ is an integer, $\tilde{A}$ is analytic in the disk $\left|t-t_{0}\right|<a$, and $\tilde{A}\left(t_{0}\right) \neq 0$. Singularities are classified as regular [114] or of the first kind [20] if $\mu=0$, and irregular or of the second kind if $\mu \geq 1$. The reason for the distinction may be seen in the example

$$
\begin{aligned}
& \dot{w}_{1}=w_{2} \\
& \dot{w}_{2}=\frac{1}{t} w_{1},
\end{aligned}
$$

which has a regular singular point at $t=0$. The system is clearly equivalent to the second order system $\ddot{w}_{1}-w_{1} / t=0$, which has an analytic solution in the disk $\left|t-t_{0}\right|<a$ given by the sum of the (obviously convergent) series

$$
w_{1}=\sum_{k=0}^{\infty} \frac{t^{k+1}}{(k !)^{2}(k+1)} .
$$

In general, linear systems with regular singularities have solutions, whereas the study of irregular singularities is much more complicated.

In order to study the behavior of a system near $t=\infty$, one makes the substitution $t=1 / \tau, z(\tau)=y(1 / \tau)$, $B(\tau)=A(1 / \tau)$. Starting with (1.4.1), we obtain

$$
\frac{d z}{d \tau}=-\frac{B(\tau)}{\tau^{2}} z
$$

Then the character of the singularity of (1.4.1) at $t=\infty$ is defined to be the character of the singularity of (1.4.2) at $\tau=0$. Specifically, if $A$ can be expressed in a Laurent series near $t=\infty$,

$$
A(t)=\sum_{n=\mu-1}^{-\infty} a_{n} t^{n}
$$

where $a_{\mu-1} \neq 0$, then the point $t=\infty$ is ordinary if $\mu<0$ and singular otherwise, regular if $\mu=0$ and irregular if $\mu \geq 1$. 


\section{CHAPTER 2}

\section{Applications to the Lorenz and Other Polynomial Systems}

As a warm up before tackling the Celestial Mechanics problem, I applied the compactification to the celebrated Lorenz system [68]. This led to some results on the Lorenz system and the characterization of a large class of complete quadratic systems, as well as the notion of a repeller at $\infty$, dual to the well-known attractor, for the Lorenz system. The work described in this Chapter has been published as $[\mathbf{4 0 , 4 1 , 4 2 ]}$.

Among the polynomial systems, quadratic systems have attracted a large amount of attention because of their important role in the mathematical sciences. These systems model diverse natural phenomenon, from fluid mechanics to the motion of the constellations $[3,4,6,7,23,24,46,47,55,56,60,65,68,69,83$, 84, 102]. They share similar features with the competing species model and the Lotka-Volterra system; see e.g., $[\mathbf{2 4}, \mathbf{5 5}, \mathbf{5 6}, \mathbf{6 0}, \mathbf{6 5}, \mathbf{7 8}, \mathbf{8 3}, \mathbf{8 4}]$. About eight hundred papers on quadratic systems are mentioned in [91]. The Lorenz system has been a celebrated quadratic system; see the original work of [68] and compare with [22], [107], and [109]. It continues in recent years to be a source for simulation and generalizations.

In this Chapter, we specialize the polynomial compactification to the Lorenz system and rescale time with $\frac{d t}{d \tau}=\left(1-R^{2}\right)\left(1+R^{2}\right)$. The resulting system extends smoothly to the boundary, which is invariant. The system on the boundary is very simple and readily solved; we introduce a new class of solutions to the Lorenz system. We then show that the boundary sphere repels inward, meaning that in the uncompactified system, infinity does, too. The dual attractor to this repeller is the much-studied strange attractor for $\rho>0$. In the second Section we define the space of Lorenz-like quadratic systems and show that they comprise most of the complete quadratic systems. We improve on the characterization given in Section 1.2 of complete systems based on the Jacobian at critical boundary points. In the final Section we relate for Lorenz-like systems the asymptotic behavior of the $t$-derivatives of $y$ with the $\tau$-derivatives of $x$. We also provide a bound on $t$-derivatives of $y$ in terms of powers of $y$.

\subsection{The Lorenz System has a Global Repeller at Infinity}

It is well known that the celebrated Lorenz system has an attractor such that every orbit ends inside a certain ellipsoid in forward time. We complement this result by a new phenomenon and by a new interpretation. We show that "infinity" is a global repeller for a set of parameters wider than that usually treated. We construct in a compacted space, a unit sphere that serves as the image of an ideal set at infinity. This sphere is shown to be the union of a family of periodic solutions. Each periodic solution is viewed as a limit cycle, or an isolated periodic orbit when restricted to a certain plane. This work was published in [40].

Historically, most studies of the Lorenz system have been for positive values of the parameters. The Lorenz system arises from a PDE in atmospheric dynamics on keeping three low order terms of a Fourier series. Lorenz originally reported chaotic behavior for $\sigma, \rho, \beta=10,28,8 / 3$ respectively. Sparrow extended this work, cataloging chaotic behavior for many positive values of the parameters, and the monograph [107] presents a substantial body of knowledge of the Lorenz system and is a testament to its important status. Numerous studies of the Lorenz system are focused on the (compact) attractor. As noted above, we applied the compactification to describe spatial infinity as a repeller for the Lorenz system. We observe that Lorenz systems are very much not typical of nonlinear systems.

By a Lorenz system $[\mathbf{6 8}]$ we mean a system satisfying

$$
\begin{aligned}
& \dot{y}_{1}=\sigma\left(y_{2}-y_{1}\right) \\
& \dot{y}_{2}=\rho y_{1}-y_{2}-y_{1} y_{3} \\
& \dot{y}_{3}=-\beta y_{3}+y_{1} y_{2},
\end{aligned}
$$

with $\sigma>0, \beta>0, \rho \in \mathbb{R}$. Note that most authors deal only with Lorenz systems with positive parameters, in which realm there is a global attractor. The existence of an attractor for $\rho \leq 0$ is a corollary of the main result of this Section: 
TheOREM 7. For the Lorenz system, "infinity" is a global repeller.

To this end we construct in a compact space, a unit sphere that serves as the image of an ideal set at infinity. This sphere is shown to be the union of a family of periodic solutions. Each periodic solution is viewed as a limit cycle, or an isolated periodic orbit when restricted to a certain plane. As a by-product of this work we provide a global picture of the vector field of the flow of the Lorenz system by augmenting the conventional analysis of its finite critical points with equilibrium points at infinity. Compare e.g. with $[60,107]$.

2.1.1. Compactification and Behavior at Infinity. To apply the compactification of Section 1.2, we will compute a little. From (1.2.9), we have

$$
\sqrt{1+4 r^{2}}=\frac{2 R}{r}-1
$$

From (1.2.8), we have

$$
\sqrt{1+4 r^{2}}=\frac{1+R^{2}}{1-R^{2}}, \quad \frac{2}{1+\sqrt{1+4 r^{2}}}=\frac{R}{r}=1-R^{2} .
$$

We also need the derivative

$$
\begin{aligned}
r \dot{r} & =y^{\dagger} \dot{y}=\sigma\left(y_{2}-y_{1}\right) y_{1}+\rho y_{1} y_{2}-y_{2}^{2}-\beta y_{3}^{2} \\
& =\frac{1}{\left(1-R^{2}\right)^{2}}\left((\sigma+\rho) x_{1} x_{2}-\sigma x_{1}^{2}-x_{2}^{2}-\beta x_{3}^{2}\right) \\
& =-\frac{S}{\left(1-R^{2}\right)^{2}},
\end{aligned}
$$

with the last equality serving to define the quadratic form $S$. Differentiating both sides of the first equation in (1.2.9) leads to

$$
\begin{aligned}
\dot{x} & =\frac{2 \dot{y}}{1+\sqrt{1+4 r^{2}}}-\frac{8 r \dot{r} y}{\sqrt{1+4 r^{2}}\left(1+\sqrt{1+4 r^{2}}\right)^{2}} \\
& =\left(1-R^{2}\right) \dot{y}-2 r \dot{r} y\left(1-R^{2}\right)^{2} \frac{1-R^{2}}{1+R^{2}} \\
& =\frac{1}{1-R^{2}}\left(\begin{array}{c}
0 \\
-x_{1} x_{3} \\
x_{1} x_{2}
\end{array}\right)+\left(\begin{array}{c}
\sigma\left(x_{2}-x_{1}\right) \\
\rho x_{1}-x_{2} \\
-\beta x_{3}
\end{array}\right)+\frac{2 S}{1+R^{2}}\left(\begin{array}{c}
x_{1} \\
x_{2} \\
x_{3}
\end{array}\right) .
\end{aligned}
$$

This equation is singular on the boundary $\partial U$, so as in Section 1.2, we rescale time with

$$
\frac{d t}{d \tau}=\left(1-R^{2}\right)\left(1+R^{2}\right)
$$

with the resulting equation for $x(\tau)$ :

$$
\left[\begin{array}{c}
x_{1}^{\prime} \\
x_{2}^{\prime} \\
x_{3}^{\prime}
\end{array}\right]=\left(1+R^{2}\right)\left[\begin{array}{c}
0 \\
-x_{1} x_{3} \\
x_{1} x_{2}
\end{array}\right]+\left(1-R^{4}\right)\left[\begin{array}{c}
\sigma\left(x_{2}-x_{1}\right) \\
\rho x_{1}-x_{2} \\
-\beta x_{3}
\end{array}\right]+2 S\left(1-R^{2}\right)\left[\begin{array}{l}
x_{1} \\
x_{2} \\
x_{3}
\end{array}\right] \text {, }
$$

where ' means $\frac{d}{d \tau}$. It is easy to show that $R$ satisfies the equation

$$
\frac{d\left(1-R^{2}\right)}{d \tau}=2 S\left(1-R^{2}\right)^{2}
$$

It is shown in [35] that given initial data, the initial value problem (2.1.2-2.1.4) possesses a unique solution on $-\infty<\tau<\infty$ such that $\|x(\tau)\| \leq 1$. In particular, it is easy to see from (2.1.4) that the boundary sphere $R=1$ is invariant. Thus we may consider the flow on the boundary. Setting $R=1$ in 
(2.1.3) reduces it to $x^{\prime}=2 f_{2}=2\left(0,-x_{1} x_{3}, x_{1} x_{2}\right)^{\dagger}$, which is readily solved. There are critical points at $( \pm 1,0,0)^{\dagger}$ and the entire circle $x_{1}=0$. The non-constant solutions are circles in $x_{2}$ and $x_{3}$, with $x_{1}$ fixed:

$$
\hat{x}(\tau)=\left(\begin{array}{c}
a \\
\sqrt{1-a^{2}} \cos (2 a \tau+\delta) \\
\sqrt{1-a^{2}} \sin (2 a \tau+\delta)
\end{array}\right)
$$

where $|a| \leq 1$, and $\delta$ is related to the starting point $\left(a, \sqrt{1-a^{2}} \cos \delta, \sqrt{1-a^{2}} \sin \delta\right)^{\dagger}$. Note that the critical points are limiting cases of the circles as $a \rightarrow \pm 1$ or 0 . For ease of visualization let us orient the axes so that $x_{1}$ "points up". Then the periodic orbits on the unit sphere may be viewed as circles of constant latitude. Note that the period is $\pi / a$ so the motion is very slow near the equator, and the equator full of critical points is a limiting case. If viewed looking down (that is, in along the positive $x_{1}$-axis), orbits in the upper hemisphere rotate counter-clockwise, and those in the lower hemisphere rotate clockwise.

Since $\|\hat{x}(\tau)\|=1, \hat{x}$ does not correspond under the compactification to anything known in the Lorenz system. However, these orbits could be interpreted to correspond to ideal solutions $y(t) \equiv \infty$ that belong to the ultra extended $\mathbb{R}^{3}$. In fact, to consider large $\|y\|$ as $\|y\| \rightarrow \infty$, we restrict our attention to the highest order terms and solve the approximate Lorenz system

$$
\begin{aligned}
& \dot{y}_{1}=0 \\
& \dot{y}_{2}=-y_{1} y_{3} \\
& \dot{y}_{3}=y_{1} y_{2},
\end{aligned}
$$

whose solution is easily seen to be (large) circles in $y_{2}$ and $y_{3}$, with $y_{1}$ constant:

$$
\hat{y}(t)=\left(\begin{array}{c}
C_{1} \\
C_{2} \cos \left(C_{1} t+\delta\right) \\
C_{2} \sin \left(C_{1} t+\delta\right)
\end{array}\right)
$$

where $C_{1}, C_{2}$, and $\delta$ define the starting point $\hat{y}(0)=\left(C_{1}, C_{2} \cos \delta, C_{2} \sin \delta\right)^{\dagger}$. The limits of these circles as $\|y\| \rightarrow \infty$ do not exist in $\mathbb{R}^{3}$, but they can be understood as orbits in the ideal set $I D$, which "bounds" $\mathbb{R}^{3}$. Let $C_{1}^{2}+C_{2}^{2}$ be large. Then these periodic vector solutions pose a certain enigma. They cannot be interpreted as natural approximations to solutions of the Lorenz system on an infinite time interval, because all solutions must enter a certain ellipsoid in forward time. We choose $C_{1}=r a$ and $C_{2}=r \sqrt{1-a^{2}}$ for $0<|a|<1$. Then as $r \rightarrow \infty, \hat{y}$ transforms under compactification to a circle on the unit sphere with constant first coordinate. Choosing instead any finite $C_{1}$ leads to a family of circles, all of which transform to the equator. Similarly, choosing a finite $C_{2}$ leads to a family of circles which transform to the poles.

We can compute the Jacobian at any point of the ball for Lorenz systems, and then specialize to the boundary sphere. We have

$$
\begin{aligned}
J_{i j}= & \frac{\partial x_{i}^{\prime}}{\partial x_{j}}=\frac{\partial}{\partial x_{j}}\left(\left(1+R^{2}\right) f_{2 i}+\left(1-R^{2}\right)\left[\left(1+R^{2}\right) f_{1 i}+2 S x_{i}\right]\right) \\
= & \frac{\partial R^{2}}{\partial x_{j}} f_{2 i}+\left(1+R^{2}\right) \frac{\partial f_{2 i}}{\partial x_{j}}-\frac{\partial R^{2}}{\partial x_{j}}\left[\left(1+R^{2}\right) f_{1 i}+2 S x_{i}\right] \\
& +\left(1-R^{2}\right)\left[\frac{\partial R^{2}}{\partial x_{j}} f_{1 i}+\left(1+R^{2}\right) \frac{\partial f_{1 i}}{\partial x_{j}}+2 \frac{\partial S}{\partial x_{j}} x_{i}+2 S \delta_{i j}\right] .
\end{aligned}
$$

This will require a few steps:

$$
\frac{\partial S}{\partial x_{j}}=\left(\begin{array}{c}
2 \sigma x_{1}-(\sigma+\rho) x_{2} \\
2 x_{2}-(\sigma+\rho) x_{1} \\
2 \beta x_{3}
\end{array}\right) \quad \frac{\partial f_{2 i}}{\partial x_{j}}=\left(\begin{array}{ccc}
0 & 0 & 0 \\
-x_{3} & 0 & -x_{1} \\
x_{2} & x_{1} & 0
\end{array}\right) \quad \frac{\partial f_{1 i}}{\partial x_{j}}=\left(\begin{array}{ccc}
-\sigma & \sigma & 0 \\
\rho & -1 & 0 \\
0 & 0 & -\beta
\end{array}\right)
$$

Parts of the first terms inside each set of square brackets in (2.1.8) cancel, with the result that the Jacobian at any point is given by

$$
2\left(\begin{array}{ccc}
0 & 0 & 0 \\
-x_{1}^{2} x_{3} & -x_{1} x_{2} x_{3} & -x_{1} x_{3}^{2} \\
x_{1}^{2} x_{2} & x_{1} x_{2}^{2} & x_{1} x_{2} x_{3}
\end{array}\right)+\left(1+R^{2}\right)\left(\begin{array}{ccc}
0 & 0 & 0 \\
-x_{3} & 0 & -x_{1} \\
x_{2} & x_{1} & 0
\end{array}\right)
$$




$$
\begin{array}{r}
-4 R^{2}\left(\begin{array}{ccc}
\sigma\left(x_{2}-x_{1}\right) x_{1} & \sigma\left(x_{2}-x_{1}\right) x_{2} & \sigma\left(x_{2}-x_{1}\right) x_{3} \\
\left(\rho x_{1}-x_{2}\right) x_{1} & \left(\rho x_{1}-x_{2}\right) x_{2} & \left(\rho x_{1}-x_{2}\right) x_{3} \\
-\beta x_{1} x_{3} & -\beta x_{2} x_{3} & -\beta x_{3}^{2}
\end{array}\right)-4 S\left(\begin{array}{ccc}
x_{1}^{2} & x_{1} x_{2} & x_{1} x_{3} \\
x_{1} x_{2} & x_{2}^{2} & x_{2} x_{3} \\
x_{1} x_{3} & x_{2} x_{3} & x_{3}^{2}
\end{array}\right) \\
+\left(1-R^{4}\right)\left(\begin{array}{ccc}
-\sigma & \sigma & 0 \\
\rho & -1 & 0 \\
0 & 0 & \beta
\end{array}\right)+2\left(1-R^{2}\right)\left(\begin{array}{ccc}
2 \sigma x_{1}^{2}-(\sigma+\rho) x_{1} x_{2}+S & 2 x_{1} x_{2}-(\sigma+\rho) x_{1}^{2} & 2 \beta x_{1} x_{3} \\
2 \sigma x_{1} x_{2}-(\sigma+\rho) x_{2}^{2} & 2 x_{2}^{2}-(\sigma+\rho) x_{1} x_{2}+S & 2 \beta x_{2} x_{3} \\
2 \sigma x_{1} x_{3}-(\sigma+\rho) x_{2} x_{3} & 2 x_{2} x_{3}-(\sigma+\rho) x_{1} x_{3} & 2 \beta x_{3}^{2}+S
\end{array}\right),
\end{array}
$$

whose trace is $\left(1-R^{2}\right)\left[\left(1+R^{2}\right)(\beta-\sigma-1)+10 S\right]$.

We first show that the system is not quasi-hyperbolic at the isolated critical points at infinity, the poles, and after that we tackle non-isolated critical points on the equator. Setting $x_{1}= \pm 1$ and $x_{2}=x_{3}=0$ in the Jacobian on the sphere yields $S=\sigma$ and

$$
J( \pm 1,0,0)=\left(\begin{array}{ccc}
0 & 0 & 0 \\
-4 \rho & 0 & \mp 2 \\
0 & \pm 2 & 0
\end{array}\right) .
$$

The characteristic polynomial is $\lambda^{3}+4 \lambda$, and the eigenvalues are $\{0,2 i,-2 i\}$. So orbits near the pole (to first order) are circles in the plane $x_{1}=a$, for any constant $a$ near \pm 1 . Evidently, the Lorenz system is not quasi-hyperbolic at the poles. Now set $x_{1}=0$ in the Jacobian on the sphere; $S=x_{2}^{2}+\beta x_{3}^{2}$ and $x_{2}^{2}+x_{3}^{2}=1$; then the Jacobian on the equator is

$$
\left(\begin{array}{ccc}
0 & -4 \sigma x_{2}^{2} & -4 \sigma x_{2} x_{3} \\
-2 x_{3} & 4(1-\beta) x_{2}^{2} x_{3}^{2} & 4(1-\beta) x_{2} x_{3}^{3} \\
2 x_{2} & 4(\beta-1) x_{2}^{3} x_{3} & 4(\beta-1) x_{2}^{2} x_{3}^{2}
\end{array}\right) .
$$

The characteristic polynomial is $\lambda^{3}$, which implies that no critical point $\infty p$, where $p$ is on the equator, is quasi-hyperbolic.

Definition. We say a surface in $\mathbb{R}^{3}$ is a periodicity surface for the system $\dot{y}=f(y)$ if it is the union of periodic orbits including critical points, and it is the maximal such object in some neighborhood of itself.

The discussion above may be summarized by:

Proposition 8. The ideal set ID is the pre-image of the boundary sphere $\partial U$, which is a periodicity surface of the compactified Lorenz system (2.1.3). The periodic orbits are circles that are limit cycles when restricted to any of the planes with $x_{1}$ fixed, $0<\left|x_{1}\right|<1$.

REMARK 9. There is great interest in Hilbert's 16th problem asking for the number of limit cycles in planar polynomial differential systems $[5,8, \mathbf{1 6}, \mathbf{2 5}, \mathbf{3 6}, \mathbf{5 8}, \mathbf{8 2}, \mathbf{1 1 8}]$. Poincare is credited with the discovery of limit cycles at infinity of planar polynomial systems $[\mathbf{8 5}, \mathbf{9 2}]$, which are not part of the official count of total limit cycles in the original Hilbert's 16th problem. It is natural now to view the set $I D$ as a periodicity surface of the Lorenz system at infinity and to ask which dynamical systems possess a periodicity surface at infinity. Much effort is invested in the classification of the finite critical points of autonomous polynomial differential systems and the classification of the critical points at infinity, as well as their phase portraits. See e.g. $[4,6]$ and their references.

2.1.2. Proof of Repulsion. If the circles on the boundary sphere can be shown to attract nearby orbits (inside the unit ball) in backwards time $\tau$, it should be possible to say something about asymptotic behavior (in backwards time $t$ ) of the Lorenz equation. This suggests limit cycles at infinity. It is easy enough to show; see e.g., [107], that all trajectories eventually enter a compact set and do not leave it. So it seems plausible that in some sense $\infty$ is a global repeller dual to the well-known attractor. On the other hand, $\frac{1}{2} \frac{d}{d t}\|y\|^{2}=-\sigma y_{1}^{2}-y_{2}^{2}-\beta y_{3}^{2}+(\sigma+\rho) y_{1} y_{2}$ takes both positive and negative values even for large $\|y\|$. Similarly, if the invariant circles on the boundary sphere are to be seen as repelling, we might hope that $R$ decreases along orbits, at least near the boundary sphere. It does not in general since $S$ takes both positive and negative values; however, it is easy to show by rotating the coordinates:

Proposition 10. For the region of parameter space $4 \sigma>(\sigma+\rho)^{2}, \beta>0, S$ is positive, and $R$ decreases along trajectories, so the boundary sphere is a global repeller. 
Proposition 10 is interesting and illustrative, but not needed for the main result of this Section, which we are ready to prove. Even though every eigenvalue of the Jacobian at every critical point on the boundary sphere of the compactified Lorenz system has real part equal to zero, we will show that the sphere repels nearby orbits.

THEOREM 11. The ideal set at infinity ID is a global repeller in the following sense: If $r\left(t_{1}\right)=\left\|y\left(t_{1}\right)\right\|$ is large enough, then there exists a $t_{2}>t_{1}$ such that $r\left(t_{1}\right)>r\left(t_{2}\right)$.

Proof. We show first that the critical points of the sphere (equator and poles) repel point-wise, and second via the Poincaré map that the circles $\hat{x}$ on the boundary repel nearby orbits. Observe that in the plane of the equator, $S=x_{2}^{2}+\beta x_{3}^{2}$, which is positive except at the origin, so that $\left(R^{2}\right)^{\prime}$ is negative. By the continuity of $S$, there is a neighborhood of the equator $\left(x_{1}\right.$ small and $x_{2}^{2}+x_{3}^{2}$ near 1$)$ on which $S$ is positive, and $\left(R^{2}\right)^{\prime}$ is negative. Thus points near the equator move in forward time toward decreasing $R$; i.e., away from the equator. Similarly, along the $x_{1}$-axis, $S=\sigma x_{1}^{2}$, so $\left(R^{2}\right)^{\prime}$ is negative, and by continuity the poles have neighborhoods all of whose points move away from the poles.

For large $r$, points $y=(0, r \cos \theta, r \sin \theta)^{\dagger}$ transform to $x=(0, R \cos \theta, R \sin \theta)^{\dagger}$ for $R \sim 1$, and points $y=(r, 0,0)^{\dagger}$ transform to $x=(R, 0,0)^{\dagger}$ for $R \sim 1$. In both cases, we have shown that $R^{\prime}<0$, so that the initial point moves away from the boundary sphere. Thus $\|x\|$ decreases, and by correspondence, $\|y\|$ decreases.

Choose $a \in(0,1)$ (the proof for $a<0$ is similar) and start with the circle $\hat{x}$ in the plane $x_{1}=a$. Choose any point on the circle as the starting point $\hat{x}(0)$. Choose a neighborhood $N$ of $\hat{x}(0)$ in the plane perpendicular to the circle at $\hat{x}(0)$. We will show that for a trajectory $x$ starting sufficiently close to the boundary sphere, at the time $\bar{\tau}$ of first return of $x$ to $N$, we have $R(\bar{\tau})<R(0)$. We can rewrite (2.1.4) in the convenient form

$$
\frac{\frac{d}{d \tau}\left(1-R^{2}\right)}{\left(1-R^{2}\right)^{2}}=2 S,
$$

which can be integrated from $\tau=0$, yielding

$$
\frac{1}{1-R^{2}(0)}-\frac{1}{1-R^{2}(\tau)}=\int_{0}^{\tau} 2 S(x(\tau)) d \tau
$$

We want to show that the left hand side is positive for $\tau=\bar{\tau}$, so it suffices to show that $\int_{0}^{\bar{\tau}} S(x(\tau)) d \tau>0$. Since we can write

$$
\int_{0}^{\bar{\tau}} S(x) d \tau=\int_{0}^{\pi / a} S(\hat{x}) d \tau+\int_{0}^{\pi / a}(S(x)-S(\hat{x})) d \tau+\int_{\pi / a}^{\bar{\tau}} S(x) d \tau
$$

our result will now follow after we show that the first integral on the right is strictly positive, that $S(x(\tau))-$ $S(\hat{x}(\tau))$ is small enough, and that the difference between $\bar{\tau}$ and $\pi / a$ is small enough.

It is easy to compute the first integral: from $(2.1 .5)$ we have

$$
\begin{aligned}
\int_{0}^{\pi / a} S(\hat{x}(\tau)) d \tau & =\int_{0}^{\pi / a}\left[\sigma a^{2}+\left(1-a^{2}\right)\left(\cos ^{2} 2 a \tau+\beta \sin ^{2} 2 a \tau\right)-(\sigma+\rho) a \sqrt{1-a^{2}} \cos 2 a \tau\right] d \tau \\
& =\frac{\pi}{a}\left[\sigma a^{2}+\frac{1}{2}\left(1-a^{2}\right)(1+\beta)\right]
\end{aligned}
$$

since the integrals of $\cos ^{2}$ and $\sin ^{2}$ over a period are equal to half the period.

The second integral in (2.1.10) is small by the continuous dependence of $x$ on initial conditions and the uniform continuity of $S$ : Given any $\delta>0$ and any finite time $T$, we can choose $\eta>0$, such that if the initial points are close together, $\|x(0)-\hat{x}(0)\|<\eta$, then for all $\tau \in[0, T],\|x(\tau)-\hat{x}(\tau)\|<\delta$. Since $S$ is uniformly continuous on the compact unit ball, given any $\epsilon>0$, we can choose $\delta>0$, such that if $\|x(\tau)-\hat{x}(\tau)\|<\delta$, then $|S(x(\tau))-S(\hat{x}(\tau))|<\epsilon$.

The difference $|\bar{\tau}-\pi / a|$ can be kept as small as desired, since we know again by the continuous dependence on initial conditions if $x(0)$ is close enough to $\hat{x}(0)$ that $x(\tau)$ stays near $\hat{x}(\tau)$ for time longer than $\pi / a$. Specifically, given $\epsilon>0$ and $T>0$, we can choose $\eta>0$, such that if $\|x(0)-\hat{x}(0)\|<\eta$, we have $\|x(\tau)-\hat{x}(\tau)\|$ stays small for all time $\tau \in[0, T]$. Choosing $T>\pi / a$ means that we can be sure $x(\tau)$ stays close to $\hat{x}(\tau)$ for $\tau=T>\pi / a$. This forces $\bar{\tau}$ to be near $\pi / a$ : $|\bar{\tau}-\pi / a|<\epsilon$. 
Now we choose $T$ enough larger than $\pi / a$ ( $T=2 \pi / a$ is plenty) that we are sure to have $T>\bar{\tau}$, and $\epsilon<\left[\sigma a^{2}+\frac{1}{2}\left(1-a^{2}\right)(\beta+1)\right] /(\pi / a+M)$, where $M$ is a bound for $S$ on the compact unit ball. Now choose $\eta$ small enough that $|S(x(\tau))-S(\hat{x}(\tau))|<\epsilon$, for all $\tau \in[0, T]$ and $|\bar{\tau}-\pi / a|<\epsilon$. Then

$$
\begin{aligned}
\int_{0}^{\bar{\tau}} S(x) d \tau & \geq \int_{0}^{\pi / a} S(\hat{x}) d \tau-\int_{0}^{\pi / a}|S(x)-S(\hat{x})| d \tau-\left|\int_{\pi / a}^{\bar{\tau}} S(x) d \tau\right| \\
& \geq \frac{\pi}{a}\left[\sigma a^{2}+\frac{1}{2}\left(1-a^{2}\right)(\beta+1)\right]-\frac{\pi \epsilon}{a}-\epsilon M \\
& >0
\end{aligned}
$$

as was to be shown. We must also show that each "circle at infinity" $\hat{y}$ as $\|\hat{y}\| \rightarrow \infty$ repels nearby points. Since $\frac{d t}{d \tau}=\left(1+R^{2}\right)\left(1-R^{2}\right)>0$ if $R^{2}<1, \tau$ increases iff $t$ increases, so it suffices to show that a sufficiently large initial point $y(0)$ transforms under compactification to a point $x(0)$ sufficiently close to the boundary sphere that the above applies. We have that $x(0)$ is the solution to $y(0)=\frac{x(0)}{1-R(0)^{2}}$. Choose $\hat{x}(0)=\frac{x(0)}{\|x(0)\|}$, then $\hat{x}(0)-x(0)=\delta \hat{x}(0)$ for some small $\delta>0$. Then

$$
\|\hat{x}(0)-x(0)\|=\delta=1-R(0)<1-R(0)^{2} \leq \frac{1-R(0)^{2}}{R(0)}=\frac{1}{\|y(0)\|},
$$

so $\|\hat{x}(0)-x(0)\|$ may be made small enough by choosing $\|y(0)\|$ large enough.

\subsection{Completeness of Quadratic Systems}

A dynamical system is called complete if every solution of it exists for all $t \in \mathbb{R}$. Let $K$ be the dimension of the vector space of quadratic systems on some $\mathbb{R}^{n}$. The subset of complete quadratic systems is shown to contain a vector subspace of dimension $2 K / 3$. We provide two proofs, one by the Gronwall lemma and the second by compactification that is capable of showing incompleteness as well. Characterization of a vector subspace of complete quadratic systems is provided. The celebrated Lorenz system for all real ranges of its parameters is shown to belong to this subspace. We also provide a sufficient condition that a system be incomplete. This work was published in [41].

It would be beneficial to modeling if we would have criteria that will inform us which dynamical systems are complete and which are not. It would be very helpful to know which systems possess global solutions for all parameters involved for all time and for all initial values. This is so for more reasons than one. On one hand, a system with solutions that blow up in finite time could be indicative of a break-down of a model. On the other hand, modeling certain phenomenon by families of differential systems that are known in advance to possess solutions that exist for all time has an obvious advantage. Criteria for systems that are not complete are of great importance as well, because it helps to know the rate of blow up.

The purpose of this study is to determine in a sense to be made precise, how large is the subspace of complete quadratic systems and to some extent to characterize this subspace. To this end we proceed with some preliminary definition and notations.

Definition. A system of differential equations $\dot{y}=f(y)$ (as well as the associated vector field $f$ ) is called complete if every solution to the system exists for all $t \in \mathbb{R}$.

Compared to the previous Section, we now extend the definition of a "Lorenz system" to mean a system satisfying

$$
\begin{aligned}
& \dot{y}_{1}=\sigma\left(y_{2}-y_{1}\right) \\
& \dot{y}_{2}=\rho y_{1}-y_{2}-y_{1} y_{3} \\
& \dot{y}_{3}=-\beta y_{3}+y_{1} y_{2}
\end{aligned}
$$

for any real values of the parameters.

Let $\mathcal{L}=\mathcal{L}\left(\mathbb{R}^{k}\right)$ be the set of quadratic systems

$$
\dot{y}=f_{2}(y)+f_{1}(y)+f_{0}, \text { with } y^{\dagger} f_{2}(y) \equiv 0,
$$

We call the autonomous system (2.2.2) a Lorenz-like system, because it generalizes certain features of the classical Lorenz system. It is easy to see that all Lorenz systems are in $\mathcal{L}\left(\mathbb{R}^{3}\right)$. 
One of the previously unanswered questions in the literature is whether or not the Lorenz system is complete for all real values of the parameters. ${ }^{1}$ We fill this gap and answer this question in the affirmative for the much larger family of systems, to be named $\mathcal{N} \mathcal{A L}$. We have not found in the literature a proof of global existence for the Lorenz system without restricting the parameters $(\sigma, \rho, \beta)$ to be positive, but our proof works for the much larger class. The earliest known completeness proof for the Lorenz system is by means of a Lyapunov function, which is not readily available for most of the Lorenz-like systems in $\mathcal{L}$. We chose as a second method of proof the compactification of Section 1.2, which is able to answer not only questions of completeness but is also able to address issues of incompleteness.

2.2.1. Completeness and Structure of Lorenz-like Systems. In this section we prove the main theorem that shows as a corollary that the Lorenz system is complete for all its real parameters. This completeness property is shared by a larger family of non autonomous quadratic systems that is denoted below by $\mathcal{N} \mathcal{A L}$.

Definition. Let $C B(\mathbb{R})$ be the family of scalar functions continuous and bounded on $\mathbb{R}$. Let $f_{2}(t, y)$ be a column vector in $\mathbb{R}^{k}$ whose components are quadratic forms: $f_{2}(t, y)_{n}=\left(y^{\dagger} f_{2 n}(t) y\right)$, with each $f_{2 n}(t)$ a lower triangular matrix with entries in $C B(\mathbb{R})$. Let $f_{1}(t, y)=f_{1}(t) y$, where $f_{1}(t)$ is a $k \times k$ matrix with entries in $C B(\mathbb{R})$, and let $f_{0}=f_{0}(t)$ be a column vector in $\mathbb{R}^{k}$ with entries in $C B(\mathbb{R})$. Then $\mathcal{N} \mathcal{A L}$ (Non-Autonomous Lorenz-like) is the class of systems

$$
\dot{y}=f_{2}(t, y)+f_{1}(t, y)+f_{0}(t), \text { with } y^{\dagger} f_{2}(t, y)=0 .
$$

The completeness of $\mathcal{N} \mathcal{A L}$ is given in the theorem below. It also includes a more detailed description of the structure of $\mathcal{L}$ that could explain the orthogonality property in (2.2.2) as a source of the completeness.

Let $\mathcal{N}$ be the linear space of all (at most) quadratic systems on $\mathbb{R}^{k}$. The main result of our study is the following.

THEOREM 12. i) All systems in $\mathcal{N} \mathcal{A L}$ are complete. ii) $\operatorname{dim}(\mathcal{L})=\frac{k}{3}(k+1)(k+2)=\frac{2}{3} \operatorname{dim}(\mathcal{N})$ and for systems in $\mathcal{L}$ the elements $f_{2 n}^{i j}$ of the lower triangular matrix $f_{2 n}$ satisfy the following relations:

$$
\begin{array}{r}
f_{2 n}^{n n}=0, \text { for } n=1,2, \ldots, k, \\
\text { for } j \neq n, f_{2 j}^{n n}+f_{2 n}^{j n}+f_{2 j}^{n j}=0 \\
\text { for } j<i<n, f_{2 n}^{i j}+f_{2 i}^{n j}+f_{2 j}^{n i}=0
\end{array}
$$

Note that in the second equation, either the second or third term is 0 because the matrix is triangular.

Proof. We provide two different proofs. The first proof is by Gronwall's lemma. By virtue of (2.2.3) we have $\frac{d\left(y^{\dagger} y\right)}{d t}=2\left[y^{\dagger} f_{1}(t, y)+y^{\dagger} f_{0}(t)\right]$. It is then easily verified that there exist two constants $m$ and $M$ such that

$$
m y^{\dagger} y \leq \frac{d\left(y^{\dagger} y\right)}{d t} \leq M y^{\dagger} y
$$

Let $y\left(t_{0}\right)$ be an initial value for (2.2.3). Then, by Gronwall's inequality or by a straightforward integration we have

$$
y^{\dagger}\left(t_{0}\right) y\left(t_{0}\right) e^{m\left(t-t_{0}\right)} \leq y^{\dagger}(t) y(t) \leq y^{\dagger}\left(t_{0}\right) y\left(t_{0}\right) e^{M\left(t-t_{0}\right)},
$$

which implies that (2.2.3) is complete.

A second proof utilizes compactification. The transformation $y(t)=\frac{x(t)}{1-x^{\dagger}(t) x(t)}$, takes $\mathbb{R}^{k}$ into the unit ball with inverse $x=\frac{2 y}{1+\sqrt{1+4 y^{\dagger} y}}$. Let $R^{2}=x^{\dagger} x$. It is easily verified [35] that with $\tilde{f}=\left(1-R^{2}\right)^{2} f_{0}+(1-$ $\left.R^{2}\right) f_{1}(x)+f_{2}(x)$, the system $(2.2 .3)$ transforms into

$$
\begin{aligned}
\frac{d x}{d t} & =\frac{\left(1+R^{2}\right) \tilde{f}\left(x,\left(1-R^{2}\right)\right)-2\left(x^{\dagger} \tilde{f}\left(x,\left(1-R^{2}\right)\right)\right) x}{\left(1+R^{2}\right)\left(1-R^{2}\right)} \\
& =\frac{1}{1-R^{2}} f_{2}+f_{1}-\frac{2 x^{\dagger} f_{1}}{1+R^{2}} x+\left(1-R^{2}\right)\left(f_{0}-\frac{2 x^{\dagger} f_{0}}{1+R^{2}} x\right),
\end{aligned}
$$

\footnotetext{
${ }^{1}$ A proof that the Lorenz system with positive parameters is "complete in forward time" (that is, that all solutions exist for all time $t>0$ ) can be found in [107] using a Lyapunov function. A proof that it is "complete in backward time" (using the same Lyapunov function) due to Meisters [74] may also be found in [22].
} 
from which we have

$$
\frac{d\left(1-R^{2}\right)}{d t}=\frac{-2\left(x^{\dagger} \tilde{f}\left(x,\left(1-R^{2}\right)\right)\right)}{\left(1+R^{2}\right)}
$$

By virtue of (2.2.3) we have

$$
x^{\dagger} \tilde{f}\left(x,\left(1-R^{2}\right)\right)=\left[\left(1-R^{2}\right) x^{\dagger} f_{0}+x^{\dagger} f_{1}(x)\right]\left(1-R^{2}\right) .
$$

Hence $1-R^{2}(t)=\left(1-R^{2}\left(t_{0}\right)\right) \exp -2 \int_{t_{0}}^{t}\left[\left(1-R^{2}\right) x^{\dagger} f_{0}+x^{\dagger} f_{1}(x)\right] d s$. It is evident that $R^{2}(t)<1$ for $t$ finite since the integrand is bounded. Thus, $\|y(t)\|<\infty$, and completeness follows.

Turning to the second part of the Theorem, let $f(y)_{n}$ be the $n^{\text {th }}$ component of $f(y)$. We can expand each component from $(2.2 .2)$,

$$
f(y)_{n}=\sum_{i=1}^{k} \sum_{j=1}^{i} f_{2 n}^{i j} y_{i} y_{j}+\sum_{i=1}^{k} f_{1 n}^{i} y_{i}+f_{0 n} .
$$

By counting parameters we see that $\operatorname{dim}(\mathcal{N})=K=\frac{k}{2}(k+1)(k+2)$, and $\mathcal{N}$ is naturally isomorphic to $\mathbb{R}^{K}$. $\mathcal{L}$ is the linear subspace defined by the condition $y^{\dagger} f_{2}=0$, which may be written as

$$
\sum_{n=1}^{k} \sum_{i=1}^{k} \sum_{j=1}^{i} f_{2 n}^{i j} y_{i} y_{j} y_{n}=0 \text {. }
$$

We collect terms and count the resulting equations. Clearly each $f_{2 i}^{i i}=0$, since each $y_{i}^{3}$ appears only once in (2.2.11); this is $k$ equations.

There are $k(k-1)$ distinct terms of the form $y_{r} y_{s}^{2}$, since there are $k$ choices of $r$ and then $k-1$ choices of $s$, yielding $k(k-1)$ equations. The constraint equations can be found by noting the values of the subscripts that cause $y_{i} y_{j} y_{n}=y_{r} y_{s}^{2}($ note $j<i)$ :

$$
\begin{aligned}
& i=r \quad \Longrightarrow \quad j=n=s \text { for } s<r \\
& j=r \quad \Longrightarrow \quad i=n=s \text { for } r<s \\
& n=r \quad \Longrightarrow \quad i=j=s .
\end{aligned}
$$

The resulting constraint equations may be written as

$$
f_{2 r}^{s s}+f_{2 s}^{r s}(\text { if } s<r)+f_{2 s}^{s r}(\text { if } r<s)=0 .
$$

There are $\left(\begin{array}{l}k \\ 3\end{array}\right)=\frac{k}{6}(k-1)(k-2)$ distinct terms of the form $y_{r} y_{s} y_{t}$, yielding $\left(\begin{array}{l}k \\ 3\end{array}\right)$ equations. We take $r<s<t$, and by the analogous analysis we can have $y_{i} y_{j} y_{n}=y_{r} y_{s} y_{t}$ as follows:

$$
\begin{aligned}
& i=t \quad \Longrightarrow \quad j=s, n=r \text { or } j=r, n=s \\
& i=s \quad \Longrightarrow \quad j=r, n=t .
\end{aligned}
$$

The resulting constraint equations may be written as

$$
f_{2 r}^{t s}+f_{2 s}^{t r}+f_{2 t}^{s r}=0 \text {. }
$$

Since there are $k+k(k-1)+\frac{k}{6}(k-1)(k-2)=\frac{k}{6}(k+1)(k+2)$ constraint equations defining $\mathcal{L}$, we have $\operatorname{dim}(\mathcal{L})=\frac{k}{3}(k+1)(k+2)$, and the ratio is $\frac{2}{3}$.

EXAMPLE 13. The case $k=2: \mathcal{N}\left(\mathbb{R}^{2}\right)$ is 12-dimensional; the general quadratic polynomial vector field on $\mathbb{R}^{2}$ has the form

$$
\left(\begin{array}{c}
f_{21}^{11} y_{1}^{2}+f_{21}^{21} y_{1} y_{2}+f_{21}^{22} y_{2}^{2}+f_{11}^{1} y_{1}+f_{11}^{2} y_{2}+f_{01} \\
f_{22}^{11} y_{1}^{2}+f_{22}^{21} y_{1} y_{2}+f_{22}^{22} y_{2}^{2}+f_{12}^{1} y_{1}+f_{12}^{2} y_{2}+f_{02}
\end{array}\right)
$$

It is helpful to view $f_{2}(y)$ (the first three summands in each row of the matrix) as the vector of quadratic forms:

$$
f(y)_{2}=\left(\begin{array}{cc}
\left(\begin{array}{ll}
y_{1} & y_{2}
\end{array}\right)\left(\begin{array}{cc}
f_{21}^{11} & 0 \\
f_{21}^{21} & f_{21}^{22}
\end{array}\right)\left(\begin{array}{l}
y_{1} \\
y_{2}
\end{array}\right) \\
\left(\begin{array}{ll}
y_{1} & y_{2}
\end{array}\right)\left(\begin{array}{cc}
f_{22}^{11} & 0 \\
f_{22}^{21} & f_{22}^{22}
\end{array}\right)\left(\begin{array}{l}
y_{1} \\
y_{2}
\end{array}\right)
\end{array}\right) .
$$


$\mathcal{L}\left(\mathbb{R}^{2}\right)$ is constrained by the condition $y^{\dagger} f_{2}=0$, which is

$$
f_{21}^{11} y_{1}^{3}+f_{21}^{21} y_{1}^{2} y_{2}+f_{21}^{22} y_{1} y_{2}^{2}+f_{22}^{11} y_{1}^{2} y_{2}+f_{22}^{21} y_{1} y_{2}^{2}+f_{22}^{22} y_{2}^{3}=0 .
$$

On collecting terms, we find four constraint equations on the coefficients:

$$
\begin{aligned}
& f_{21}^{11}=f_{22}^{22}=0 \\
& f_{21}^{22}+f_{22}^{21}=0 \\
& f_{22}^{11}+f_{21}^{21}=0
\end{aligned}
$$

so the quadratic forms reduce to

$$
f_{2}=\left(\begin{array}{c}
\left(\begin{array}{ll}
y_{1} & y_{2}
\end{array}\right)\left(\begin{array}{cc}
0 & 0 \\
a & b
\end{array}\right)\left(\begin{array}{l}
y_{1} \\
y_{2}
\end{array}\right) \\
\left(\begin{array}{ll}
y_{1} & y_{2}
\end{array}\right)\left(\begin{array}{ll}
-a & 0 \\
-b & 0
\end{array}\right)\left(\begin{array}{l}
y_{1} \\
y_{2}
\end{array}\right)
\end{array}\right),
$$

and the general system in $\mathcal{L}$ has the form:

$$
\left(\begin{array}{c}
a y_{1} y_{2}+b y_{2}^{2}+f_{11}^{1} y_{1}+f_{11}^{2} y_{2}+f_{01} \\
-a y_{1}^{2}-b y_{1} y_{2}+f_{12}^{1} y_{1}+f_{12}^{2} y_{2}+f_{02}
\end{array}\right)
$$

where $a$ and $b$ are the free parameters $a=f_{21}^{21}=-f_{22}^{11}$ and $b=f_{21}^{22}=-f_{22}^{21}$.

2.2.2. Some incomplete systems. It goes without saying that the determination whether a quadratic system is incomplete is also a problem of considerable importance. In the sequel we will make a few observations regarding such systems and conclude with the formulation of a theorem that will demonstrate why compactification is central to proving incompleteness.

Under the isomorphism mentioned in the proof of theorem, $\mathcal{N}$ inherits an inner product, the Euclidean inner product on $\mathbb{R}^{K}$, allowing us to define $\mathcal{L}^{\perp}$, the orthogonal complement of $\mathcal{L} \subset \mathcal{N} . \mathcal{L}\left(\mathbb{R}^{2}\right)^{\perp}$ is fourdimensional and spanned by the basis elements:

$$
\left(\begin{array}{c}
y_{1}^{2} \\
0
\end{array}\right), \quad\left(\begin{array}{c}
0 \\
y_{2}^{2}
\end{array}\right), \quad\left(\begin{array}{c}
y_{1} y_{2} \\
y_{1}^{2}
\end{array}\right), \quad\left(\begin{array}{c}
y_{2}^{2} \\
y_{1} y_{2}
\end{array}\right) .
$$

The first two are readily seen to represent the incomplete systems

$$
\begin{gathered}
\dot{y}_{1}=y_{1}^{2} \\
\dot{y}_{2}=0
\end{gathered} \text { and } \begin{gathered}
\dot{y}_{1}=0 \\
\dot{y}_{2}=y_{2}^{2}
\end{gathered} \text {. }
$$

The linear span of the third basis element is the set of systems of the form

$$
\begin{gathered}
\dot{y}_{1}=c y_{1} y_{2} \\
\dot{y}_{2}=c y_{1}^{2},
\end{gathered}
$$

for any constant $c$. If $c \neq 0$, then the system with initial condition $y(0)=(1 / c, 1 / c)^{\dagger}$ has a solution $y_{1}(t)=y_{2}(t)=\frac{1}{c(1-t)}$, which cannot be extended to $t=1$. The span of the fourth basis element may be analyzed similarly.

EXAMPLE 14 . The case $k=3: \mathcal{N}\left(\mathbb{R}^{3}\right)^{\perp}$ is ten-dimensional and spanned by the basis elements:

$$
\begin{gathered}
\left(\begin{array}{c}
y_{1}^{2} \\
0 \\
0
\end{array}\right), \quad\left(\begin{array}{c}
0 \\
y_{2}^{2} \\
0
\end{array}\right), \quad\left(\begin{array}{c}
0 \\
0 \\
y_{3}^{2}
\end{array}\right), \quad\left(\begin{array}{c}
y_{1} y_{2} \\
y_{1}^{2} \\
0
\end{array}\right), \quad\left(\begin{array}{c}
y_{2}^{2} \\
y_{1} y_{2} \\
0
\end{array}\right), \\
\left(\begin{array}{c}
y_{1} y_{3} \\
0 \\
y_{1}^{2}
\end{array}\right), \quad\left(\begin{array}{c}
y_{3}^{2} \\
0 \\
y_{1} y_{3}
\end{array}\right), \quad\left(\begin{array}{c}
0 \\
y_{2} y_{3} \\
y_{2}^{2}
\end{array}\right), \quad\left(\begin{array}{c}
0 \\
y_{3}^{2} \\
y_{2} y_{3}
\end{array}\right), \quad\left(\begin{array}{l}
y_{2} y_{3} \\
y_{1} y_{3} \\
y_{1} y_{2}
\end{array}\right) .
\end{gathered}
$$

As for $k=2$, the first three are easily seen to represent incomplete systems, and the next six are similar to the third and fourth elements from the $k=2$ example. The linear span of the final basis element is the set 
of systems of the form

$$
\begin{aligned}
& \dot{y}_{1}=c y_{2} y_{3} \\
& \dot{y}_{2}=c y_{1} y_{3} \\
& \dot{y}_{3}=c y_{1} y_{2},
\end{aligned}
$$

for $c$ any constant. If $c \neq 0$, then the system with initial condition $y(0)=(1 / c, 1 / c, 1 / c)^{\dagger}$ has a solution $y_{1}(t)=y_{2}(t)=y_{3}(t)=\frac{1}{c(1-t)}$, which cannot be extended to $t=1$.

These examples may be generalized as a proposition.

Proposition 15. The linear space $\mathcal{L}^{\perp}=\mathcal{L}\left(\mathbb{R}^{k}\right)^{\perp}$ has a basis all of whose elements represent incomplete systems.

PROOF. In terms of the quadratic forms $f_{2 n}, \mathcal{L}^{\perp}$ is the span of the following:

$$
\begin{aligned}
& \left\{f_{2 n}^{n n}=1\right\}_{n=1,2, \ldots, k}, \\
& \left\{f_{2 n}^{i i}=f_{2 i}^{n i}=1\right\}_{i<n=1,2, \ldots, k}, \\
& \left\{f_{2 n}^{i i}=f_{2 i}^{i n}=1\right\}_{n<i=1,2, \ldots, k} \\
& \left\{f_{2 n}^{i j}=f_{2 j}^{i n}=f_{2 i}^{j n}=1\right\}_{n<j<i} .
\end{aligned}
$$

This translates into a basis for $\mathcal{L}^{\perp}$ in terms of systems of equations:

$$
\left\{\dot{y}_{n}=y_{n}^{2}\right\}_{n=1,2, \ldots, k},\left\{\begin{array}{l}
\dot{y}_{i}=y_{i} y_{n} \\
\dot{y}_{n}=y_{i}^{2}
\end{array}\right\}_{i \neq n=1,2, \ldots, k}, \quad\left\{\begin{array}{l}
\dot{y}_{n}=y_{j} y_{i} \\
\dot{y}_{j}=y_{n} y_{i} \\
\dot{y}_{i}=y_{n} y_{j} .
\end{array}\right\}_{n<j<i}
$$

The specific systems given in the examples for $k=2$ and $k=3$ above work in the general case. Note as well that any system in the linear span (excluding the trivial system) of the first $k$ basis elements is not complete.

Broadening the focus now to polynomial systems, the fact that compactification is central to understanding completeness as well as incompleteness is seen from the following theorem. We cannot see how the Gronwall lemma can be used to prove incompleteness.

THEOREM 16. Given the polynomial system $\dot{y}=f(y)$, let $p$ be a critical point of the compactified system

$$
\frac{d x}{d \tau}=\left(1+R^{2}\right) \tilde{f}-2\left(x^{\dagger} \tilde{f}\right) x
$$

such that $p^{\dagger} p=1$. Consider the Jacobian of the compacted system about these critical points. A necessary condition for a polynomial dynamical system to be complete is that the eigenvalues of the Jacobians about all these critical points be purely imaginary.

Proof. Suppose $p$ is a critical point of the compactified system (2.2.12) at which the Jacobian has $r>0$ eigenvalues $\alpha_{1}, \ldots, \alpha_{r}$ with negative real parts. [If the real parts of the eigenvalues $\alpha_{1}, \ldots, \alpha_{r}$ are positive, then the argument to follow can be made to the time-reversed flow instead]. Then per Chapter IX of [48], there is an $r$-parameter family of solutions $x(\tau)$ that approach the critical point exponentially; for every solution in the family there is a $\tau_{0}$ such that for all $\tau \geq \tau_{0}$

$$
\|x(\tau)-p\| \leq b_{1} e^{-m \tau}
$$

for some constant $b_{1} \geq 0$ and $m>0$. Let us turn to the quantity $1-R^{2}$. Near $p,\|p\|=1$,

$$
\begin{aligned}
1-R^{2}(\tau) & =1-\sum_{i=1}^{n}\left(x_{i}-p_{i}+p_{i}\right)^{2}=-\sum_{i=1}^{n}\left[2 p_{i}\left(x_{i}-p_{i}\right)+\left(x_{i}-p_{i}\right)^{2}\right] \\
& \leq 2\|x(\tau)-p\|+\|x(\tau)-p\|^{2} \\
& \leq b_{2} e^{-m \tau}
\end{aligned}
$$

for some $b_{2} \geq 0$ and all $\tau \geq \tau_{0}$. Corresponding to the family of solutions of the compactified system, there is an $r$-parameter family of solutions of the uncompactified system. Suppose a solution of this family exists 
on $\left[t_{0}, t_{\max }\right)$ where the initial point $t_{0}$ corresponds to $\tau=0$, and $t_{\max } \leq \infty$. We will show that $t_{\max }$ is finite. Recall that by inverting $\frac{d \tau}{d t}$ we have

$$
t_{\max }-t_{0}=\int_{0}^{\infty}\left(1-R^{2}\right)^{L-1}\left(1+R^{2}\right) d \eta=I_{1}+I_{2}
$$

where

$$
I_{1}:=\int_{0}^{\tau_{0}}\left(1-R^{2}\right)^{L-1}\left(1+R^{2}\right) d \eta, I_{2}:=\int_{\tau_{0}}^{\infty}\left(1-R^{2}\right)^{L-1}\left(1+R^{2}\right) d \eta .
$$

By the continuity of $1-R^{2}(\tau)$ on $\left[0, \tau_{0}\right]$ we conclude that $I_{1}$ is bounded. On the interval $\left[\tau_{0}, \infty\right)$ we have by (2.2.14) that

$$
I_{2}=\int_{\tau_{0}}^{\infty}\left(1-R^{2}\right)^{L-1}\left(1+R^{2}\right) d \eta \leq \int_{\tau_{0}}^{\infty} 2 b_{2} e^{-(L-1) m \tau} d \eta=\frac{2 b_{2}}{(L-1) m} e^{-m \tau_{0}} .
$$

This concludes the proof that $t_{\max }$ is finite.

The completeness property is expected to be the exception rather than the rule in polynomial systems of high degree. It would be nice to characterize all the nonlinear polynomial differential systems that are incomplete. The examples above suggest that the "majority" of elements (perhaps all nontrivial elements) in $\mathcal{L}\left(\mathbb{R}^{k}\right)^{\perp}$ are incomplete. See [35] for some incompleteness results for generic polynomial $k$-dimensional systems using compactification.

A differential system need not belong to $\mathcal{N} \mathcal{A} \mathcal{L}$ in order to be complete, as the following planar quadratic example shows. Let $p\left(y_{2}\right)$ be a linear function and $q\left(y_{2}\right)$ a quadratic function of $y_{2}$. Let $a, b$ be real numbers. Then the system

$$
y_{1}^{\prime}=p\left(y_{2}\right) y_{1}+q\left(y_{2}\right), y_{2}^{\prime}=a y_{1}+b .
$$

is a complete quadratic system, but does not necessarily belong to $\mathcal{L}$ or to $\mathcal{N} \mathcal{A L}$.

REMARK 17. Completeness could be beneficial to the study of singularities of a dynamical system in the complex plane $[\mathbf{1 0 6}, \mathbf{1 0 9}]$. In particular, $[\mathbf{1 0 9}]$ entails the computation of infinitely many coefficients of a Laurent or $\psi$-series expansion. Because of the completeness of systems in $\mathcal{L}$, our analysis predicts that any such expansion for any real parameters must involve complex coefficients. This is corroborated for the Lorenz system by $[\mathbf{1 0 9}]$.

\subsection{Fields of Lorenz-like Systems Near $\infty$}

We study by differential geometry the neighborhood of the unit sphere at infinity and derive conclusions on Lorenz-like systems. We study the vector fields $\frac{d^{l} y}{d t^{l}}, l=1,2, \ldots$, for systems in $\mathcal{L}$ when the norm of $y$ is large and becomes unbounded. We apply this knowledge to differential geometry for trajectories far from the origin and show that the mutually orthogonal triplet of differential geometry $-T, N, B-$ is near that of the compactified system, and that both triplets approach that of the strictly second-degree system $\dot{y}=f_{2}(y)$. Lastly, we show a similar relationship between higher order derivatives of systems in $\mathcal{L}$ and their compactifications.

A main purpose of our analysis is to show how the behavior of the compactified system on the boundary sphere indicates behavior of solutions and their derivatives of the original system for large $\|y\|$. This is helpful since the compactified system near $R=1$ is usually much simpler than the original system. The asymptotic behavior of the order of growth of the higher derivatives; $\frac{d^{l} y}{d t^{l}}, l=0,1,2, \ldots$ follows, under appropriate conditions, as a bonus from a formula that provides the asymptotic directions of the derivatives. This result was published in $[\mathbf{4 2}]$ with a sketch of the proof, which is provided here in full.

We first exhibit the relevance of the highest-order system $\dot{y}=f_{2}(y)$ to the original system through analysis of the moving triple $T, N, B$ of unit tangent, normal, and binormal vectors. We can show that $T_{y}, N_{y}, B_{y}$ for trajectories in $\mathcal{L}\left(\mathbb{R}^{3}\right)$ and those of the compactified trajectories $T_{x}, N_{x}, B_{x}$ approach those of $\dot{y}=f_{2}$ as $R \rightarrow 1$. The continuity properties of the compactified differential equation on the compact unit ball then imply that for large $\|y\|, y$ looks a lot like the solutions of $\dot{y}=f_{2}(y)$. In fact, the relevance of the compactified system extends to all orders of derivatives. We stress that we do not expect such correspondence of vector fields for systems not in $\mathcal{L}$. 
We assume that the system $\dot{y}=f(y), f \in \mathcal{L}$ is compactified as above, and the time is rescaled via $\frac{d t}{d \tau}=\left(1-R^{2}\right)\left(1+R^{2}\right)$, and the compactified, rescaled system is

$$
x^{\prime}=\left(1+R^{2}\right) f_{2}+\left(1-R^{2}\right)\left[\left(1+R^{2}\right) f_{1}-2\left(x^{\dagger} f_{1}\right) x\right]+\left(1-R^{2}\right)^{2}\left[\left(1+R^{2}\right) f_{0}-2\left(x^{\dagger} f_{0}\right) x\right] .
$$

From (2.3.1), we can write the equation for $R^{2}$,

$$
\begin{aligned}
\frac{1}{2}\left(R^{2}\right)^{\prime}= & x^{\dagger} x^{\prime}=\left(1-R^{2}\right)\left(\left(1+R^{2}\right) x^{\dagger} f_{1}-2 x^{\dagger} f_{1} R^{2}\right) \\
& +\left(1-R^{2}\right)^{2}\left(\left(1+R^{2}\right) x^{\dagger} f_{0}-2 x^{\dagger} f_{0} R^{2}\right) \\
= & \left(1-R^{2}\right)^{2}\left(x^{\dagger} f_{1}+\left(1-R^{2}\right) x^{\dagger} f_{0}\right) \\
\left(1-R^{2}\right)^{\prime}= & 2 S\left(1-R^{2}\right)^{2}, \text { with } S=-x^{\dagger} f_{1}-\left(1-R^{2}\right) x^{\dagger} f_{0} .
\end{aligned}
$$

For the strictly second degree polynomial system $\dot{y}=f_{2}(y)$ in $\mathcal{L}\left(\mathbb{R}^{3}\right)$, a direct calculation shows that

$$
T_{2}=\frac{f_{2}}{\left\|f_{2}\right\|}, \quad N_{2}=\frac{\left\|f_{2}\right\|^{2}\left[D f_{2}\right] f_{2}-\left(f_{2}^{\dagger}\left[D f_{2}\right] f_{2}\right) f_{2}}{\|\| f_{2}\left\|^{2}\left[D f_{2}\right] f_{2}-\left(f_{2}^{\dagger}\left[D f_{2}\right] f_{2}\right) f_{2}\right\|}, \quad B_{2}=\frac{f_{2} \times\left(\left[D f_{2}\right] f_{2}\right)}{\left\|f_{2} \times\left(\left[D f_{2}\right] f_{2}\right)\right\|},
$$

where we use the notation $\left[D f_{i}\right]$ for the Jacobian of the vector function $f_{i}$. The expressions in (2.3.3) are given as functions of the variable $y$; however, it is easy to see that the values of the expressions are not changed if they are expressed in terms of $x$. Unless otherwise indicated, all subsequent occurrences of $f_{i}$ and $\left[D f_{i}\right]$ are to be understood as $f_{i}(x)$ and $\left[D f_{i}(x)\right]$.

Theorem 18. Let $y \in \mathcal{L}\left(\mathbb{R}^{3}\right)$. Then the triples $T_{y}, N_{y}, B_{y}$ and $T_{x}, N_{x}, B_{x}$ coincide with $T_{2}, N_{2}, B_{2}$, plus terms of order $O\left(\frac{1-R^{2}}{\left\|f_{2}\right\|}\right)$ as $R^{2} \rightarrow 1$, away from the zeros of $f_{2}$.

Proof. To compute the first order terms of the moving triple for $x$ and for $y$, we will need an expression for $1 /\left\|x^{\prime}\right\|$.

$$
\left\|x^{\prime}\right\|^{2}=\left(1+R^{2}\right)^{2}\left\|f_{2}\right\|^{2}+2\left(1+R^{2}\right)^{2}\left(1-R^{2}\right) f_{1}^{\dagger} f_{2}+O\left(1-R^{2}\right)^{2} .
$$

From this we can approximate

$$
\frac{1}{\left\|x^{\prime}\right\|} \sim \frac{1}{\left(1+R^{2}\right)\left\|f_{2}\right\|}\left(1-\left(1-R^{2}\right) \frac{f_{1}^{\dagger} f_{2}}{\left\|f_{2}\right\|^{2}}\right)+O\left(\frac{\left(1-R^{2}\right)^{2}}{\left\|f_{2}\right\|^{2}}\right), \quad \text { as } R^{2} \rightarrow 1 .
$$

Now since $T_{x}=x^{\prime} /\left\|x^{\prime}\right\|$ we have

$$
T_{x}=\frac{f_{2}}{\left\|f_{2}\right\|}+\frac{\left(1-R^{2}\right)}{\left\|f_{2}\right\|}\left(f_{1}-\frac{2\left(x^{\dagger} f_{1}\right) x}{1+R^{2}}-\frac{f_{1}^{\dagger} f_{2}}{\left\|f_{2}\right\|^{2}} f_{2}\right)+O\left(\frac{\left(1-R^{2}\right)^{2}}{\left\|f_{2}\right\|^{2}}\right) \quad \text { as } R^{2} \rightarrow 1 .
$$

The first term on the right is $T_{2}$.

To compute $T_{y}$, we start with $y=\left(1-R^{2}\right)^{-1} x$ and differentiate

$$
\begin{aligned}
\dot{y} & =\frac{d y}{d \tau} \frac{d \tau}{d t} \\
& =\frac{\left(\left(1-R^{2}\right)^{-1}\right)^{\prime} x+\left(1-R^{2}\right)^{-1} x^{\prime}}{\left(1-R^{2}\right)\left(1+R^{2}\right)} \\
& =\frac{-2 S x+\left(1-R^{2}\right)^{-1}\left[\left(1+R^{2}\right) f_{2}+\left(1-R^{2}\right)\left(\left(1+R^{2}\right) f_{1}-2\left(x^{\dagger} f_{1}\right) x\right)\right]}{\left(1-R^{2}\right)\left(1+R^{2}\right)}+O\left(1-R^{2}\right) \\
& =\left(1-R^{2}\right)^{-2}\left(f_{2}+\left(1-R^{2}\right) f_{1}\right)+O\left(1-R^{2}\right),
\end{aligned}
$$

the third equality follows from (2.3.1) \& (2.3.2), and the fourth from the fact that $S+x^{\dagger} f_{1} \sim O\left(1-R^{2}\right)$. The unit tangent vector $T_{y}$ to a trajectory is $\dot{y}$ divided by its magnitude:

$$
T_{y}=\frac{f_{2}+\left(1-R^{2}\right) f_{1}}{\left\|f_{2}+\left(1-R^{2}\right) f_{1}\right\|}+O\left(1-R^{2}\right)^{2} .
$$

Now the square of the denominator is

$$
\left\|f_{2}\right\|^{2}+2\left(1-R^{2}\right) f_{1}^{\dagger} f_{2}+O\left(1-R^{2}\right)^{2} .
$$


It follows that we can approximate $1 /\left\|f_{2}+\left(1-R^{2}\right) f_{1}\right\|$ as

$$
\frac{1}{\left\|f_{2}\right\|}\left(1-\left(1-R^{2}\right) \frac{f_{1}^{\dagger} f_{2}}{\left\|f_{2}\right\|^{2}}\right)+O\left(\frac{\left(1-R^{2}\right)^{2}}{\left\|f_{2}\right\|^{2}}\right)
$$

so that

$$
T_{y} \sim \frac{1}{\left\|f_{2}\right\|}\left[f_{2}+\left(1-R^{2}\right)\left(f_{1}-\frac{f_{1}^{\dagger} f_{2}}{\left\|f_{2}\right\|^{2}} f_{2}\right)\right]+O\left(\frac{\left(1-R^{2}\right)^{2}}{\left\|f_{2}\right\|^{2}}\right)
$$

Putting it together, we have

$$
T_{y}=T_{x}+\frac{2\left(1-R^{2}\right)\left(x^{\dagger} f_{1}\right) x}{\left(1+R^{2}\right)\left\|f_{2}\right\|}+O\left(\frac{\left(1-R^{2}\right)^{2}}{\left\|f_{2}\right\|^{2}}\right) .
$$

It follows that both $T_{y}$ and $T_{x}$ approach $T_{2}$ as $\left(1-R^{2}\right) /\left\|f_{2}\right\| \rightarrow 0$.

Now we compute the low order terms of $N_{x}$ and $N_{y}$. First we need

$$
\begin{aligned}
x^{\prime \prime}= & \left(1+R^{2}\right) f_{2}^{\prime}+\left(1-R^{2}\right)\left[\left(1+R^{2}\right) f_{1}^{\prime}-2\left(x^{\dagger} f_{1}\right)^{\prime} x-2\left(x^{\dagger} f_{1}\right) x^{\prime}\right] \\
= & \left(1+R^{2}\right)^{2}\left[D f_{2}\right] f_{2}+\left(1-R^{2}\right)\left(1+R^{2}\right)\left[D f_{2}\right]\left(\left(1+R^{2}\right) f_{1}-2\left(x^{\dagger} f_{1}\right) x\right) \\
& +\left(1-R^{2}\right)\left[\left(1+R^{2}\right)^{2}\left[D f_{1}\right] f_{2}-2\left(x^{\dagger} f_{1}\right)^{\prime} x-2\left(1+R^{2}\right)\left(x^{\dagger} f_{1}\right) f_{2}\right]+O\left(1-R^{2}\right)^{2},
\end{aligned}
$$

since $f_{i}^{\prime}=\left[D f_{i}\right] x^{\prime}$. Now $N_{x}$ is the unit vector in the direction $\left\|x^{\prime}\right\|^{2} x^{\prime \prime}-x^{\prime \dagger} x^{\prime \prime} x^{\prime}$. From (2.3.4), using $\left[D f_{i}\right] x=i f_{i}$, we can write

$$
\begin{gathered}
\left\|x^{\prime}\right\|^{2} x^{\prime \prime}=\left(1+R^{2}\right)^{4}\left[D f_{2}\right] f_{2}+\left(1-R^{2}\right)\left(1+R^{2}\right)^{3} \times \\
\left\{\left\|f_{2}\right\|^{2}\left[\left(1+R^{2}\right)\left(\left[D f_{1}\right] f_{2}+\left[D f_{2}\right] f_{1}\right)-6\left(x^{\dagger} f_{1}\right) f_{2}-2\left(f_{2}^{\dagger} f_{1}\right) x-2\left(x^{\dagger}\left[D f_{1}\right] f_{2}\right) x\right]+2\left(f_{2}^{\dagger} f_{1}\right)\left[D f_{2}\right] f_{2}\right\}
\end{gathered}
$$

plus higher order terms. The zero order term of $x^{\prime \dagger} x^{\prime \prime}$ is $\left(1+R^{2}\right)^{3} f_{2}^{\dagger}\left[D f_{2}\right] f_{2}$ (which vanishes for the Lorenz system), and the first order term is

$$
\left(1-R^{2}\right)\left(1+R^{2}\right)^{2}\left(\left(1+R^{2}\right)\left(f_{2}^{\dagger}\left[D f_{1}\right] f_{2}+f_{2}^{\dagger}\left[D f_{2}\right] f_{1}\right)-4\left\|f_{2}\right\|^{2}\left(x^{\dagger} f_{1}\right)+\left(1+R^{2}\right) f_{1}^{\dagger}\left[D f_{2}\right] f_{2}\right)
$$

Putting the pieces together, the direction for $N_{x}$ is

$$
\begin{aligned}
& \left(1+R^{2}\right)\left\|f_{2}\right\|^{2}\left[D f_{2}\right] f_{2}-\left(f_{2}^{\dagger}\left[D f_{2}\right] f_{2}\right) f_{2}+\left(1-R^{2}\right) \times \\
& \left\{\left\|f_{2}\right\|^{2}\left[\left(1+R^{2}\right)\left(\left[D f_{1}\right] f_{2}+\left[D f_{2}\right] f_{1}\right)-4\left(x^{\dagger} f_{1}\right) f_{2}-2 f_{2}^{\dagger} f_{1} x-2\left(x^{\dagger}\left[D f_{1}\right] f_{2}\right) x\right]\right. \\
& \left.+2\left(f_{2}^{\dagger} f_{1}\right)\left[D f_{2}\right] f_{2}-\left(1+R^{2}\right)\left(f_{2}^{\dagger}\left[D f_{1}\right] f_{2}+f_{2}^{\dagger}\left[D f_{2}\right] f_{1}\right) f_{2}-\left(1+R^{2}\right)\left(f_{1}^{\dagger}\left[D f_{2}\right] f_{2}\right) f_{2}\right\},
\end{aligned}
$$

which matches $N_{2}$ to lowest order. 
$N_{y}$ is the unit vector in the direction $n_{y}=\|\dot{y}\|^{2} \ddot{y}-\dot{y}^{\dagger} \ddot{y} \dot{y}$. From (2.3.5), we have

$$
\begin{aligned}
\left(1-R^{2}\right)\left(1+R^{2}\right) \ddot{y}= & {\left[f_{2}+\left(1-R^{2}\right) f_{1}\right]^{\prime}\left(1-R^{2}\right)^{-2}+\left[f_{2}+\left(1-R^{2}\right) f_{1}\right]\left[\left(1-R^{2}\right)^{-2}\right]^{\prime} } \\
= & \left(\left[D f_{2}\right] x^{\prime}+\left(1-R^{2}\right)\left[D f_{1}\right] x^{\prime}-2\left(x^{\dagger} f_{1}\right)\left(1-R^{2}\right)^{2} f_{1}\right)\left(1-R^{2}\right)^{-2} \\
& +\left[f_{2}-\left(1-R^{2}\right) f_{1}\right] 4\left(x^{\dagger} f_{1}\right)\left(1-R^{2}\right)^{-1} \\
= & {\left[D f_{2}\right]\left[\left(1+R^{2}\right) f_{2}+\left(1-R^{2}\right)\left(\left(1+R^{2}\right) f_{1}-2\left(x^{\dagger} f_{1}\right) x\right)\right]\left(1-R^{2}\right)^{-2}-2\left(x^{\dagger} f_{1}\right) f_{1} } \\
& +\left[D f_{1}\right]\left[\left(1+R^{2}\right) f_{2}+\left(1-R^{2}\right)\left(\left(1+R^{2}\right) f_{1}-2\left(x^{\dagger} f_{1}\right) x\right)\right]\left(1-R^{2}\right)^{-1} \\
& +4\left(x^{\dagger} f_{1}\right) f_{2}\left(1-R^{2}\right)^{-1}+4\left(x^{\dagger} f_{1}\right) f_{1} \\
= & \frac{1+R^{2}}{\left(1-R^{2}\right)^{2}}\left[D f_{2}\right] f_{2}+\frac{1+R^{2}}{1-R^{2}}\left(\left[D f_{2}\right] f_{1}+\left[D f_{1}\right] f_{2}\right) \\
& +\frac{-2\left(x^{\dagger} f_{1}\right)\left[D f_{2}\right] x}{1-R^{2}}+2\left(x^{\dagger} f_{1}\right) f_{1}+\left(1+R^{2}\right)\left[D f_{1}\right] f_{1} \\
& -2\left(x^{\dagger} f_{1}\right)\left[D f_{1}\right] x+\frac{4\left(x^{\dagger} f_{1}\right) f_{2}}{1-R^{2}} \\
\left(1-R^{2}\right)^{3} \ddot{y}= & {\left[D f_{2}\right] f_{2}+\left(1-R^{2}\right)\left(\left[D f_{1}\right] f_{2}+\left[D f_{2}\right] f_{1}\right)+O\left(1-R^{2}\right)^{2} } \\
\left(1-R^{2}\right)^{7}\|\dot{y}\|^{2} \ddot{y}= & \left\|f_{2}\right\|^{2}\left[D f_{2}\right] f_{2}+\left(1-R^{2}\right)\left(2 f_{1}^{\dagger} f_{2}\left[D f_{2}\right] f_{2}+\left\|f_{2}\right\|^{2}\left(\left[D f_{1}\right] f_{2}+\left[D f_{2}\right] f_{1}\right)\right) \\
& +O\left(1-R^{2}\right)^{2} .
\end{aligned}
$$

For the other part of $n_{y}$,

$$
\begin{gathered}
\left(1-R^{2}\right)^{5} \dot{y}^{\dagger} \ddot{y}=f_{2}^{\dagger}\left[D f_{2}\right] f_{2}+\left(1-R^{2}\right)\left(-\left(x^{\dagger} f_{1}\right)\left(f_{1}^{\dagger}\left[D f_{2}\right] f_{2}\right)+\left(f_{2}^{\dagger}\left[D f_{1}\right] f_{2}+f_{2}^{\dagger}\left[D f_{2}\right] f_{1}\right)\right)+O\left(1-R^{2}\right) \\
\left(1-R^{2}\right)^{7} \dot{y}^{\dagger} \ddot{y} \dot{y}=\left(f_{2}^{\dagger}\left[D f_{2}\right] f_{2}\right) f_{2}+\left(1-R^{2}\right)\left(f_{2}^{\dagger}\left[D f_{1}\right] f_{2}+f_{2}^{\dagger}\left[D f_{2}\right] f_{1}+f_{1}^{\dagger}\left[D f_{2}\right] f_{2}\right) f_{2}+O\left(1-R^{2}\right)^{2},
\end{gathered}
$$

so that

$$
\begin{aligned}
&\left(1-R^{2}\right)^{7} n_{y}=\left\|f_{2}\right\|^{2}\left[D f_{2}\right] f_{2}-\left(f_{2}^{\dagger}\left[D f_{2}\right] f_{2}\right) f_{2}+\left(1-R^{2}\right) \\
& \times\left\{2 f_{1}^{\dagger} f_{2}\left[D f_{2}\right] f_{2}+\left\|f_{2}\right\|^{2}\left(\left[D f_{1}\right] f_{2}+\left[D f_{2}\right] f_{1}\right)\right. \\
&\left.\quad-\left(f_{2}^{\dagger}\left[D f_{1}\right] f_{2}+f_{2}^{\dagger}\left[D f_{2}\right] f_{1}\right) f_{2}-\left(f_{1}^{\dagger}\left[D f_{2}\right] f_{2}\right) f_{2}\right\} \\
&+O\left(1-R^{2}\right)^{2}
\end{aligned}
$$

It follows that both $N_{y}$ and $N_{x}$ approach $N_{2}$ as $\left(1-R^{2}\right) \rightarrow 0$. Since $B=T \times N$, we also have that $B_{y}$ and $B_{x}$ approach $B_{2}$.

We develop an interesting relationship between $t$-derivatives of $y$ for large $\|y\|$ and $\tau$-derivatives of $x$ near the boundary sphere, but away from critical points and zeros of higher derivatives. We show that for systems in $\mathcal{L}$, the vector fields of higher derivatives of $y$ with respect to $t$ have the same direction as the corresponding derivatives of $x$ with respect to $\tau$ for $\|y\|$ large enough. We stress that this property need not hold for general quadratic systems.

Proposition 19. Let $y \in \mathcal{L}$. Then, for all integers $n \geq 0$, as $\|y\| \rightarrow \infty$ or $R^{2} \rightarrow 1^{-}$, we have

$$
\left(1-R^{2}\right)^{n+1}\left(1+R^{2}\right)^{n} d^{n} y / d t^{n}=d^{n} x / d \tau^{n}+\mathcal{O}\left(1-R^{2}\right) .
$$

Moreover, the order of growth of the derivatives $d^{n} y / d t^{n}$ is given by

$$
\left\|d^{n} y / d t^{n}\right\| \leq M_{n}\|y\|^{n+1}, n=0,1,2, \ldots,
$$

where $M_{n}$ are certain constants. Furthermore, for each $n$, let $S_{n}$ be the set of points in the boundary where $d^{n} x / d \tau^{n}=0$. Then $\forall \epsilon>0$, there is a neighborhood $U_{n}$ of $S_{n}$ in the closed unit ball, with $m\left(U_{n}\right)<\epsilon$, such that for $x \in$ the complement of $U_{n}$ and $\|y\| \rightarrow \infty$ or $R^{2} \rightarrow 1^{-}$we have

$$
\frac{d^{n} y / d t^{n}}{\left\|d^{n} y / d t^{n}\right\|}=\frac{d^{n} x / d \tau^{n}}{\left\|d^{n} x / d \tau^{n}\right\|}+\mathcal{O}\left(1-R^{2}\right) .
$$


Proof. We first prove (2.3.8) by induction. The conclusions in (2.3.9) and (2.3.10) follow from (2.3.8) and are left as an exercise to the reader. The case $n=0$ follows from the definition of $x$, and we suppose the formula holds for $n-1$. Leibniz' rule gives

$$
\frac{d^{n} x}{d t^{n}}=\frac{d^{n}}{d t^{n}}\left[\left(1-R^{2}\right) y\right]=\sum_{i=0}^{n}\left(\begin{array}{c}
n \\
i
\end{array}\right) \frac{d^{n-i}\left(1-R^{2}\right)}{d t^{n-i}} \frac{d^{i} y}{d t^{i}}
$$

where $\left(\begin{array}{l}n \\ i\end{array}\right)$ is the binomial coefficient $\frac{n !}{i !(n-i) !}$. Separating the two terms for $i=n$ and $i=n-1$ from the sum gives

$$
\left(1-R^{2}\right) \frac{d^{n} y}{d t^{n}}=\frac{d^{n} x}{d t^{n}}-n \frac{d\left(1-R^{2}\right)}{d t} \frac{d^{n-1} y}{d t^{n-1}}-\sum_{i=0}^{n-2}\left(\begin{array}{c}
n \\
i
\end{array}\right) \frac{d^{n-i}\left(1-R^{2}\right)}{d t^{n-i}} \frac{d^{i} y}{d t^{i}} .
$$

The induction hypothesis allows substitution for all the derivatives of $y$ on the right, but we need two additional results, both of which are shown by induction.

First, for $n \geq 0$, we have

$$
\left(1-R^{2}\right)^{n}\left(1+R^{2}\right)^{n} \frac{d^{n} x}{d t^{n}}=\frac{d^{n} x}{d \tau^{n}}+\left(1-R^{2}\right) D_{n-1} x,
$$

where $D_{n}$ is an $n^{\text {th }}$ degree polynomial in $\frac{d}{d \tau}$, with rational (in $x$ ) continuous coefficients in the unit ball.

The cases $n=0$ and 1 are trivial; $D_{-1}$ and $D_{0}$ are identically zero. Now assume $n>1$. Note that

$$
\frac{d}{d \tau}\left(1-R^{2}\right)^{n}=2 n S\left(1-R^{2}\right)^{n+1} .
$$

So we have by the induction hypothesis

$$
\begin{aligned}
\frac{d^{n} x}{d t^{n}}= & \frac{d}{d \tau}\left(\frac{d^{n-1} x}{d t^{n-1}}\right) \frac{d \tau}{d t} \\
= & \frac{d}{d \tau}\left[\left(1-R^{2}\right)^{1-n}\left(1+R^{2}\right)^{1-n}\left(\frac{d^{n-1} x}{d \tau^{n-1}}+\left(1-R^{2}\right) D_{n-2} x\right)\right]\left(1-R^{2}\right)^{-1}\left(1+R^{2}\right)^{-1} \\
= & \left(1-R^{2}\right)^{-n}\left(1+R^{2}\right)^{-n}\left[\frac{d^{n} x}{d \tau^{n}}+2 S\left(1-R^{2}\right)^{2} D_{n-2} x+\left(1-R^{2}\right) \frac{d\left(D_{n-2} x\right)}{d \tau}\right] \\
& +2(n-1) S R^{2}\left(1-R^{2}\right)^{1-n}\left(1+R^{2}\right)^{-n-1}\left[\frac{d^{n-1} x}{d \tau^{n-1}}+\left(1-R^{2}\right) D_{n-2} x\right] \\
\left(1-R^{2}\right)^{n}\left(1+R^{2}\right)^{n} \frac{d^{n} x}{d t^{n}}= & \frac{d^{n} x}{d \tau^{n}}+\left(1-R^{2}\right)\left[2 S\left(1-R^{2}\right) D_{n-2} x+\frac{d\left(D_{n-2} x\right)}{d \tau}\right] \\
& +\frac{2(n-1) S R^{2}\left(1-R^{2}\right)}{1+R^{2}}\left[\frac{d^{n-1} x}{d \tau^{n-1}}+\left(1-R^{2}\right) D_{n-2} x\right] \\
= & \frac{d^{n} x}{d \tau^{n}}+\left(1-R^{2}\right) D_{n-1} x,
\end{aligned}
$$

where

$$
D_{n-1} x=2 S\left(1-R^{2}\right) D_{n-2} x+\frac{d\left(D_{n-2} x\right)}{d \tau}+\frac{2(n-1) S R^{2}}{1+R^{2}}\left[\frac{d^{n-1} x}{d \tau^{n-1}}+\left(1-R^{2}\right) D_{n-2} x\right] .
$$

It is easy to see that $D_{n-1}$ is as claimed.

Second, for $n \geq 1$,

$$
\left(1-R^{2}\right)^{n-2}\left(1+R^{2}\right)^{2 n-1} \frac{d^{n}\left(1-R^{2}\right)}{d t^{n}}=P_{n}(x),
$$

where $P_{n}(x)$ is a polynomial in $x$.

The case $n=1$ is easy:

$$
\frac{d\left(1-R^{2}\right)}{d t}=\frac{d\left(1-R^{2}\right)}{d \tau}\left(1-R^{2}\right)^{-1}\left(1+R^{2}\right)^{-1}=\frac{2 S\left(1-R^{2}\right)}{1+R^{2}},
$$


so that

and we take $P_{1}=2 S$. Now assume $n \geq 2$. Then we have

$$
\left(1-R^{2}\right)^{-1}\left(1+R^{2}\right) \frac{d\left(1-R^{2}\right)}{d t}=2 S
$$

$$
\begin{aligned}
\frac{d^{n}\left(1-R^{2}\right)}{d t^{n}} & =\frac{d}{d \tau}\left(\frac{d^{n-1}\left(1-R^{2}\right)}{d t^{n-1}}\right) \frac{d \tau}{d t} \\
& =\frac{d}{d \tau}\left[\left(1-R^{2}\right)^{3-n}\left(1+R^{2}\right)^{3-2 n} P_{n-1}(x)\right] \div\left(1-R^{2}\right)\left(1+R^{2}\right),
\end{aligned}
$$

by the induction hypothesis. The $\tau$-derivative via the product rule is

$$
\begin{aligned}
& \quad(3-n) 2 S\left(1-R^{2}\right)^{4-n}\left(1+R^{2}\right)^{3-2 n} P_{n-1}(x) \\
& +\left(1-R^{2}\right)^{3-n}(-2 S)(3-2 n)\left(1+R^{2}\right)^{2-2 n}\left(1-R^{2}\right)^{2} P_{n-1}(x) \\
& +\left(1-R^{2}\right)^{3-n}\left(1+R^{2}\right)^{3-2 n} \frac{d P_{n-1}(x)}{d \tau} \\
& =\left(1-R^{2}\right)^{3-n}\left(1+R^{2}\right)^{2-2 n} P_{n}(x),
\end{aligned}
$$

from which the result follows immediately. Note that if $n=3$, the first of the three terms in the above sum (before the $=$ ) is not present, but this does not affect the proof.

Returning to (2.3.11), we substitute using the induction hypothesis, as well as (2.3.12 \& 2.3.16):

$$
\begin{aligned}
\left(1-R^{2}\right) \frac{d^{n} y}{d t^{n}} & =\frac{\frac{d^{n} x}{d \tau^{n}}+\left(1-R^{2}\right) D_{n-1} x}{\left(1-R^{2}\right)^{n}\left(1+R^{2}\right)^{n}}-\frac{2 S\left(1-R^{2}\right)}{1+R^{2}} \frac{n \frac{d^{n-1} x}{d \tau^{n-1}}+\left(1-R^{2}\right) D_{n-2} x}{\left(1-R^{2}\right)^{n}\left(1+R^{2}\right)^{n-1}} \\
& -\sum_{i=0}^{n-2}\left(\begin{array}{c}
n \\
i
\end{array}\right) \frac{\left(1+R^{2}\right)^{1-2 n+2 i}}{\left(1-R^{2}\right)^{n-i-2}} P_{n-i}(x) \frac{\frac{d^{i} x}{d \tau^{i}}+\left(1-R^{2}\right) D_{i} x}{\left(1-R^{2}\right)^{i+1}\left(1+R^{2}\right)^{i}} .
\end{aligned}
$$

Multiplying through by $\left(1-R^{2}\right)^{n}\left(1+R^{2}\right)^{n}$ completes the proof.

REMARK 20. The reason that Lorenz-like systems distinguish themselves from other nonlinear systems so that the vector fields $\frac{d^{l} x}{d \tau^{l}}$ and $\frac{d^{l} y}{d t^{l}}, l=0,1,2, \ldots$ are asymptotically parallel to each other for large $\|y\|$ can be traced back to the relation (2.3.13), which again is a result of the orthogonality in (2.2.2). Recall that orthogonality featured also in the completeness result. It basically says that in a Lorenz-Like system $y^{\dagger} f(y)$ does not grow faster than $\|y\|^{2}$ as $\|y\| \rightarrow \infty$.

For the Lorenz system or any system with $x^{\prime}$ proportional to $\left(0,-x_{3}, x_{2}\right)^{\dagger}$, it is easy to see that

$$
T_{\hat{x}}=\frac{1}{\sqrt{x_{2}^{2}+x_{3}^{2}}}\left(\begin{array}{c}
0 \\
-x_{3} \\
x_{2}
\end{array}\right), \quad N_{\hat{x}}=\frac{1}{\sqrt{x_{2}^{2}+x_{3}^{2}}}\left(\begin{array}{c}
0 \\
-x_{2} \\
-x_{3}
\end{array}\right), \quad B_{\hat{x}}=\left(\begin{array}{l}
1 \\
0 \\
0
\end{array}\right) .
$$

Theorem 18 says that the triple $T, N, B$ for an orbit of the Lorenz system and of its compactified version approach those of (2.3.17) as $R^{2} \rightarrow 1$ :

Corollary 21. The moving triple T, N, B for the Lorenz system and the compactified version coincide with those of the circle $\hat{x}$, plus terms of order $O\left(\frac{\left(1-R^{2}\right)}{\left\|x_{1}\right\| \sqrt{x_{2}^{2}+x_{3}^{2}}}\right)$ as $R^{2} \rightarrow 1$. 


\section{CHAPTER 3}

\section{Celestial Mechanics at $\infty$ via Compactification}

In this Chapter, we extend the compactification of Section 1.2 beyond polynomial systems to the equations of celestial mechanics, analyze the behavior of the compactified system in the boundary, and investigate solutions to some approximate systems near the boundary. In Section 3.1, we develop the differential equation for the compactified version of (1.0.1) and show that the compactification does not introduce any critical points in the open unit ball, which means, as in the polynomial case, that the topological structures of the flows on the open ball and on all of Euclidean space should be identical. The compactified system can be extended to the boundary points, and and we show in fact that all critical points and singular points of the system are on the boundary sphere.

In Section 3.2, we study the system restricted to the boundary sphere, where the behavior is less complicated, and exact solutions can be given in closed form. These boundary solutions represent new objects in Celestial Mechanics, as the compactification of trajectories at $\infty$. In the absence of collisions, these solutions on the boundary approach the critical point set, which is the unit $3 N-1$-sphere where $x_{N+i}=0$. These solutions offer insight into solutions near the boundary, in particular suggesting a set of approximating systems near the boundary. In Section 3.3, we determine properties of solutions to two approximating systems, both of which can be solved exactly, and one of these solutions is asymptotic to solutions of the compactified system. The uncompactification of the second approximate solution will be shown to be asymptotic to the solutions we give in Chapter 5 to Celestial Mechanics equation.

\subsection{Compactifying the N-Body Problem}

In this Section, we develop the equations of motion for the compactified N-body problem and show that the compactification does not introduce any critical points in the open unit ball, which means that the topological structures of the flows on the open ball and on all of Euclidean space should be identical. We compute time derivatives of interesting quantities and find an expression for the evolution of the distance of $x$ from the boundary. We show that if a trajectory experiences a singularity as $t \rightarrow \sigma^{-}$, then the compactified trajectory approaches the boundary sphere. Even though we don't expect analytic solutions to the compactified system, it will prove useful to investigate series coefficients as if there were analytic solutions.

We make the second-order equation for the $q_{i}$ (1.0.1) into a $6 N$-dimensional system in the usual way: for $i=1, \ldots, N$, let $y_{i}=q_{i}$, and $y_{N+i}=\dot{q}_{i}$, where for each $i, y_{i}$ and $y_{N+i}$ are vectors in $\mathbb{R}^{3}$. Then we can express the system as $\dot{y}=f(y)$, with the right hand side serving to define $f(y)$ :

$$
\begin{aligned}
\dot{y}_{i} & =y_{N+i} \\
\dot{y}_{N+i} & =\sum_{j \neq i} \frac{m_{j}\left(y_{j}-y_{i}\right)}{\left\|y_{i}-y_{j}\right\|^{3}} .
\end{aligned}
$$

Our compactified version of the original system of equations will be obtained on substituting $y=\frac{x}{1-x^{\dagger} x}$. All of $\mathbb{R}^{6 N}$ is mapped to the open unit ball $\mathbb{B}^{6 N}$, and the pre-image of its boundary $\mathbb{S}^{6 N-1}$ is an ideal set bounding $\mathbb{R}^{6 N}$, which may be identified with all directions at infinity. Compactifications in general, and this one in particular, make it possible to augment the conventional set of solutions of a differential system with an ideal set of solutions $y \equiv \infty$. Their geometric realization is given by trajectories on $\mathbb{S}^{6 N-1}$ viewed as the boundary sphere of the unit ball in $\mathbb{R}^{6 N}$. These ideal trajectories are solutions to simplified systems of differential equations. Indeed, these ideal solutions, as shown in this Chapter, provide a wealth of information on the solutions of $\dot{y}=f(y)$ for $\|y\|$ large. 
As in Chapter 2, we compactify with $y=\kappa x$, with $\kappa=\frac{1}{1-R^{2}}$, where $R=\|x\|$. Then

$$
\dot{x}=\frac{\dot{y}}{\kappa}-(\nabla \kappa)^{\dagger} \dot{y} \frac{y}{\kappa^{2}}=\kappa^{-1}\left[f(y)-(\nabla \kappa)^{\dagger} f(y) \frac{y}{\kappa}\right],
$$

where the gradient is taken with respect $y$. Squaring both sides of the transformation equation $y=\frac{x}{1-R^{2}}$ gives $r^{2}=\frac{R^{2}}{\left(1-R^{2}\right)^{2}}$, with $r=\|y\|$, or

$$
r^{2} R^{4}-\left(2 r^{2}+1\right) R^{2}+r^{2}=0
$$

which leads to $R^{2}=\left(2 r^{2}+1 \pm \sqrt{1+4 r^{2}}\right) / 2 r^{2}$. Choosing the negative root ensures $R \in[0,1]$. Then

$$
\kappa=\frac{1}{1-R^{2}}=\frac{1}{2}\left(1+\sqrt{1+4 r^{2}}\right) .
$$

For future reference, we provide the transformation equations between $y \in \mathbb{R}^{6 N}$ and $x \in \mathbb{B}^{6 N}$ based on $y=\kappa x$ :

$$
y=\frac{x}{1-R^{2}} \quad x=\frac{2 y}{1+\sqrt{1+4 r^{2}}} .
$$

It will be useful to solve (3.1.3) for the radical:

$$
\sqrt{1+4 r^{2}}=\frac{2}{1-R^{2}}-1=\frac{1+R^{2}}{1-R^{2}} \text {. }
$$

We compute the gradient (with respect to $y$ ) from (3.1.3)

$$
\nabla \kappa=\frac{1}{2} \frac{1}{2} \frac{1}{\sqrt{1+4 r^{2}}} 4 \nabla r^{2}=\frac{1}{\sqrt{1+4 r^{2}}} 2 y=\frac{2 y\left(1-R^{2}\right)}{1+R^{2}}=\frac{2 x}{1+R^{2}} .
$$

Then

$$
(\nabla \kappa)^{\dagger} f(y)=\frac{2}{1+R^{2}} x^{\dagger} f(y)=\frac{2}{1+R^{2}} \sum_{k=1}^{N} x_{N+k}^{\dagger}\left(\frac{x_{k}}{1-R^{2}}+\left(1-R^{2}\right)^{2} \sum_{j \neq k} \frac{m_{j}\left(x_{j}-x_{k}\right)}{\left\|x_{k}-x_{j}\right\|^{3}}\right),
$$

so that the evolution of the compactified system in the open unit ball $\mathbb{B}^{6 N}$ is governed by

$$
\begin{gathered}
\dot{x}_{i}=x_{N+i}-\frac{2}{1+R^{2}} \sum_{k=1}^{N} x_{N+k}^{\dagger}\left(x_{k}+\left(1-R^{2}\right)^{3} \sum_{j \neq k} \frac{m_{j}\left(x_{j}-x_{k}\right)}{\left\|x_{k}-x_{j}\right\|^{3}}\right) x_{i} \\
\dot{x}_{N+i}=-\frac{2}{1+R^{2}} \sum_{k=1}^{N} x_{N+k}^{\dagger}\left(x_{k}+\left(1-R^{2}\right)^{3} \sum_{j \neq k} \frac{m_{j}\left(x_{j}-x_{k}\right)}{\left\|x_{k}-x_{j}\right\|^{3}}\right) x_{N+i} \\
+\left(1-R^{2}\right)^{3} \sum_{k \neq i} \frac{m_{k}\left(x_{k}-x_{i}\right)}{\left\|x_{i}-x_{k}\right\|^{3}} .
\end{gathered}
$$

Just as with (3.1.1), the right hand side of this system is analytic on $\mathbb{B}^{6 N} \backslash \Delta$, meaning that given any noncollision point; i.e., any point $\xi \in \mathbb{B}^{6 N} \backslash \Delta$, the right-hand side of (3.1.6) is expandable in a power series in $x-\xi$, convergent for $\|x-\xi\|$ sufficiently small. This does not of course imply that there are analytic solutions to this system.

Let us define some short hand formulas:

$g_{i j}=\left\|x_{i}-x_{j}\right\|, \quad h=\sum_{k=1}^{N} x_{k}^{\dagger} x_{N+k}, \quad G_{k}=\sum_{j \neq k} \frac{m_{j}\left(x_{j}-x_{k}\right)}{g_{k j}^{3}}, \quad L=\sum_{k=1}^{N} x_{N+k}^{\dagger} G_{k}, \quad \theta=-2 \frac{h+\left(1-R^{2}\right)^{3} L}{1+R^{2}}$.

These identities follow easily from the definitions:

$$
\begin{aligned}
G_{k} & =\left(1-R^{2}\right)^{2} \dot{y}_{N+k}=\left(1-R^{2}\right)^{2} \ddot{q}_{k} \\
L & =\left(1-R^{2}\right)^{3} \sum_{k=1}^{N} y_{N+k}^{\dagger} \dot{y}_{N+k}=\frac{1}{2}\left(1-R^{2}\right)^{3} \frac{d}{d t} \sum_{k=1}^{N} y_{N+k}^{\dagger} y_{N+k}=\frac{1}{2}\left(1-R^{2}\right)^{3} \frac{d}{d t} \dot{q}^{\dagger} \dot{q} .
\end{aligned}
$$


The short hand allows us to express the system as

$$
\begin{aligned}
\dot{x}_{i} & =\theta x_{i}+x_{N+i} \\
\dot{x}_{N+i} & =\left(1-R^{2}\right)^{3} G_{i}+\theta x_{N+i},
\end{aligned}
$$

or

$$
\dot{x}=\left(\begin{array}{cc}
\theta I_{3 N} & I_{3 N} \\
O_{3 N} & \theta I_{3 N}
\end{array}\right) x+\left(\begin{array}{c}
0_{3 N} \\
\left(1-R^{2}\right)^{3} G
\end{array}\right),
$$

where $G=\left(G_{1}^{\dagger}, \ldots, G_{N}^{\dagger}\right)^{\dagger}$.

We have seen in Section 1.2 that the compactification of a polynomial system does not introduce any critical points in the interior, and it's true here as well. If the right hand side of (3.1.2) vanished, we would have

$$
f(y)-(\nabla \kappa)^{\dagger} f(y) x=0,
$$

to which we apply $(\nabla \kappa)^{\dagger}$ yielding

$$
(\nabla \kappa)^{\dagger} f(y)\left[1-\frac{(\nabla \kappa)^{\dagger} y}{\kappa}\right]=0
$$

Since $\nabla \kappa=\frac{2 x}{1+R^{2}}$, and $f(y) \neq 0$, since the original problem has no critical points, the quantity in the brackets must vanish, so

$$
\frac{2 R^{2}}{1+R^{2}}=\frac{2 x^{\dagger} x}{1+R^{2}}=\frac{(\nabla \kappa)^{\dagger} y}{\kappa}=1,
$$

which requires $R^{2}=1$, and $x$ must be on the boundary. Thus

Lemma 22. The compactified system (3.1.7) has no critical points in the interior of $\mathbb{B}^{6 N}$.

By the existence and uniqueness theorems, the system (3.1.7) has a unique solution through any $x\left(t_{0}\right)=$ $x_{0} \in \overline{\mathbb{B}^{6 N}} \backslash \Delta$, the non-collision points of the closed ball. If $x_{0}$ corresponds under the compactification to $y_{0}=(q(0), \dot{q}(0))^{\dagger}$, then the solution $x(t)$ exists for a maximal interval $t \in\left[t_{0}, \sigma\right)$, with $\sigma$ defined in Section 1.1 .

Consider the system (3.1.7) expressed in terms of $\phi_{i}=\left(1-R^{2}\right)^{3} G_{i}$ as a further shorthand:

$$
\begin{aligned}
\dot{x_{i}} & =\theta x_{i}+x_{N+i} \\
\dot{x}_{N+i} & =\theta x_{N+i}+\phi_{i},
\end{aligned}
$$

or

$$
\dot{x}=\left(\begin{array}{cc}
\theta I_{3 N} & I_{3 N} \\
O_{3 N} & \theta I_{3 N}
\end{array}\right) x+\left(\begin{array}{c}
0_{3 N} \\
\phi
\end{array}\right),
$$

viewing $\phi=\left(\phi_{1}^{\dagger}, \ldots, \phi_{N}^{\dagger}\right)^{\dagger}$ as a non-homogeneous element. We will now record a formal solution for (3.1.8) based on the technique of multiplying by the integrating factor

$$
\exp \left[-\int_{0}^{t}\left(\begin{array}{cc}
\theta(s) I_{3 N} & I_{3 N} \\
O_{3 N} & \theta(s) I_{3 N}
\end{array}\right) d s\right] .
$$

First note that

$$
\begin{gathered}
\exp \left[\int_{0}^{t}\left(\begin{array}{cc}
\theta(s) I_{3 N} & I_{3 N} \\
O_{3 N} & \theta(s) I_{3 N}
\end{array}\right) d s\right]=\exp \left[\int_{0}^{t} \theta(s) d s I_{6 N}+\int_{0}^{t}\left(\begin{array}{cc}
O_{3 N} & I_{3 N} \\
O_{3 N} & O_{3 N}
\end{array}\right) d s\right] \\
=\exp \int_{0}^{t} \theta(s) d s I_{6 N} \exp \int_{0}^{t}\left(\begin{array}{cc}
O_{3 N} & I_{3 N} \\
O_{3 N} & O_{3 N}
\end{array}\right) d s=e^{\int_{0}^{t} \theta(s) d s} \exp \left(\begin{array}{cc}
O_{3 N} & t I_{3 N} \\
O_{3 N} & O_{3 N}
\end{array}\right) \\
=e^{\int_{0}^{t} \theta(s) d s}\left(\begin{array}{cc}
I_{3 N} & t I_{3 N} \\
O_{3 N} & I_{3 N}
\end{array}\right) .
\end{gathered}
$$

On multiplying, we have

$$
\frac{d}{d t}\left\{\exp \left[-\int_{0}^{t}\left(\begin{array}{cc}
\theta(s) I_{3 N} & I_{3 N} \\
O_{3 N} & \theta(s) I_{3 N}
\end{array}\right) d s\right] x\right\}=-\left(\begin{array}{cc}
\theta I_{3 N} & I_{3 N} \\
O_{3 N} & \theta I_{3 N}
\end{array}\right) \exp \left[-\int_{0}^{t}\left(\begin{array}{cc}
\theta(s) I_{3 N} & I_{3 N} \\
O_{3 N} & \theta(s) I_{3 N}
\end{array}\right) d s\right] x
$$


or

$$
\begin{gathered}
+\exp \left[-\int_{0}^{t}\left(\begin{array}{cc}
\theta(s) I_{3 N} & I_{3 N} \\
O_{3 N} & \theta(s) I_{3 N}
\end{array}\right) d s\right] \dot{x} \\
=\exp \left[-\int_{0}^{t}\left(\begin{array}{cc}
\theta(s) I_{3 N} & I_{3 N} \\
O_{3 N} & \theta(s) I_{3 N}
\end{array}\right) d s\right]\left(\begin{array}{c}
0_{3 N} \\
\phi
\end{array}\right),
\end{gathered}
$$

$$
\frac{d}{d t}\left\{e^{-\int_{0}^{t} \theta(s) d s}\left(\begin{array}{cc}
I_{3 N} & -t I_{3 N} \\
O_{3 N} & I_{3 N}
\end{array}\right) x\right\}=e^{-\int_{0}^{t} \theta(s) d s}\left(\begin{array}{cc}
I_{3 N} & -t I_{3 N} \\
O_{3 N} & I_{3 N}
\end{array}\right)\left(\begin{array}{c}
0_{3 N} \\
\phi
\end{array}\right) .
$$

So we have the formal solution

$$
\begin{aligned}
x & =e^{\int_{0}^{t} \theta(s) d s}\left(\begin{array}{cc}
I_{3 N} & t I_{3 N} \\
O_{3 N} & I_{3 N}
\end{array}\right)\left\{x(0)+\int_{0}^{t} e^{-\int_{0}^{\lambda} \theta(\nu) d \nu}\left(\begin{array}{cc}
I_{3 N} & -\lambda I_{3 N} \\
O_{3 N} & I_{3 N}
\end{array}\right)\left(\begin{array}{c}
0_{3 N} \\
\phi(\lambda)
\end{array}\right) d \lambda\right\} \\
& =e^{\int_{0}^{t} \theta(s) d s}\left\{\int_{0}^{t} e^{-\int_{0}^{\lambda} \theta(\nu) d \nu}\left(\begin{array}{c}
(t-\lambda) I_{3 N} \\
I_{3 N}
\end{array}\right) \phi(\lambda) d \lambda+\left(\begin{array}{cc}
I_{3 N} & t I_{3 N} \\
O_{3 N} & I_{3 N}
\end{array}\right) x(0)\right\} \\
& =\int_{0}^{t} e^{\int_{\lambda}^{t} \theta(s) d s}\left(\begin{array}{c}
(t-\lambda) I_{3 N} \\
I_{3 N}
\end{array}\right) \phi(\lambda) d \lambda+e^{\int_{0}^{t} \theta(s) d s}\left(\begin{array}{cc}
I_{3 N} & t I_{3 N} \\
O_{3 N} & I_{3 N}
\end{array}\right) x(0) .
\end{aligned}
$$

3.1.1. Time derivatives. We evaluate the time derivatives of the short-hand quantities defined just before (3.1.7) above.

$$
\begin{gathered}
\dot{h}=\sum_{k=1}^{N}\left\{x_{k}^{\dagger} \dot{x}_{N+k}+x_{N+k}^{\dagger} \dot{x}_{k}\right\}=\sum_{k=1}^{N}\left\{x_{k}^{\dagger}\left[\theta x_{N+k}+\left(1-R^{2}\right)^{3} G_{k}\right]+x_{N+k}^{\dagger}\left[\theta x_{k}+x_{N+k}\right]\right\} \\
=2 \theta h+A+\left(1-R^{2}\right)^{3} \sum_{k=1}^{N} x_{k}^{\dagger} G_{k},
\end{gathered}
$$

where

$$
A=\sum_{k=1}^{N} x_{N+k}^{\dagger} x_{N+k}
$$

And we have

$$
\dot{A}=2 \sum_{k=1}^{N} x_{N+k}^{\dagger}\left[\theta x_{N+k}+\left(1-R^{2}\right)^{3} G_{k}\right]=2 \theta A+2\left(1-R^{2}\right)^{3} L .
$$

If we define analogously to $L$

$$
K=\sum_{k=1}^{N} x_{k}^{\dagger} G_{k}
$$

then we can express

$$
\dot{h}=2 \theta h+A+\left(1-R^{2}\right)^{3} K .
$$

To find $\dot{L}$ and $\dot{K}$, we start with

so

$$
\begin{gathered}
\frac{d}{d t} g_{j k}^{2}=2\left(x_{j}-x_{k}\right)^{\dagger}\left(\dot{x}_{j}-\dot{x}_{k}\right)=2\left(x_{j}-x_{k}\right)^{\dagger}\left[\theta\left(x_{j}-x_{k}\right)+x_{N+j}-x_{N+k}\right] \\
=2 \theta g_{j k}^{2}+2\left(x_{j}-x_{k}\right)^{\dagger}\left(x_{N+j}-x_{N+k}\right)
\end{gathered}
$$

and we can compute

$$
\begin{gathered}
\frac{d}{d t} g_{j k}^{-3}=-\frac{3}{2}\left(g_{j k}^{2}\right)^{-5 / 2} \frac{d}{d t} g_{j k}^{2}=-\frac{3}{2 g_{j k}^{5}}\left[2 \theta g_{j k}^{2}+2\left(x_{j}-x_{k}\right)^{\dagger}\left(x_{N+j}-x_{N+k}\right)\right] \\
=-\frac{3 \theta}{g_{j k}^{3}}-\frac{3}{g_{j k}^{5}}\left(x_{j}-x_{k}\right)^{\dagger}\left(x_{N+j}-x_{N+k}\right)
\end{gathered}
$$

$$
\dot{G}_{k}=\sum_{j \neq k} m_{j}\left\{\left(x_{j}-x_{k}\right) \frac{d}{d t} g_{j k}^{-3}+\frac{\dot{x}_{j}-\dot{x}_{k}}{g_{j k}^{3}}\right\}
$$




$$
\begin{gathered}
=\sum_{j \neq k} m_{j}\left\{-\left[\frac{3 \theta}{g_{j k}^{3}}+\frac{3}{g_{j k}^{5}}\left(x_{j}-x_{k}\right)^{\dagger}\left(x_{N+j}-x_{N+k}\right)\right]\left(x_{j}-x_{k}\right)+\frac{\theta\left(x_{j}-x_{k}\right)+x_{N+j}-x_{N+k}}{g_{j k}^{3}}\right\} \\
=\sum_{j \neq k} m_{j}\left\{-\left[\frac{2 \theta}{g_{j k}^{3}}+\frac{3}{g_{j k}^{5}}\left(x_{j}-x_{k}\right)^{\dagger}\left(x_{N+j}-x_{N+k}\right)\right]\left(x_{j}-x_{k}\right)+\frac{x_{N+j}-x_{N+k}}{g_{j k}^{3}}\right\} \\
=\sum_{j \neq k} \frac{m_{j}}{g_{j k}^{3}}\left\{-2 \theta\left(x_{j}-x_{k}\right)+\left[I_{3}-\frac{3}{g_{j k}^{2}}\left(x_{j}-x_{k}\right)\left(x_{j}-x_{k}\right)^{\dagger}\right]\left(x_{N+j}-x_{N+k}\right)\right\} \\
=-2 \theta G_{k}+\sum_{j \neq k} \frac{m_{j}}{g_{j k}^{3}}\left[I_{3}-\frac{3}{g_{j k}^{2}}\left(x_{j}-x_{k}\right)\left(x_{j}-x_{k}\right)^{\dagger}\right]\left(x_{N+j}-x_{N+k}\right) .
\end{gathered}
$$

Now we have

$$
\begin{gathered}
\dot{L}=\sum_{k=1}^{N}\left\{G_{k}^{\dagger} \dot{x}_{N+k}+x_{N+k}^{\dagger} \dot{G}_{k}\right\}=\sum_{k=1}^{N}\left\{G_{k}^{\dagger}\left[\theta x_{N+k}+\left(1-R^{2}\right)^{3} G_{k}\right]+x_{N+k}^{\dagger} \dot{G}_{k}\right\} \\
=\theta L+\left(1-R^{2}\right)^{3} G^{2}+\sum_{k=1}^{N} x_{N+k}^{\dagger} \dot{G}_{k}, \\
=-\theta L+\left(1-R^{2}\right)^{3} G^{2}+\sum_{k=1}^{N} x_{N+k}^{\dagger} \sum_{j \neq k} \frac{m_{j}}{g_{j k}^{3}}\left[I_{3}-\frac{3}{g_{j k}^{2}}\left(x_{j}-x_{k}\right)\left(x_{j}-x_{k}\right)^{\dagger}\right]\left(x_{N+j}-x_{N+k}\right),
\end{gathered}
$$

where

$$
G^{2}=\sum_{k=1}^{N} G_{k}^{\dagger} G_{k}
$$

Moreover,

$$
\dot{K}=\sum_{k=1}^{N}\left\{G_{k}^{\dagger} \dot{x}_{k}+x_{k}^{\dagger} \dot{G}_{k}\right\}=\sum_{k=1}^{N}\left\{G_{k}^{\dagger}\left[\theta x_{k}+x_{N+k}\right]+x_{k}^{\dagger} \dot{G}_{k}\right\}=\theta K+L+\sum_{k=1}^{N} x_{k}^{\dagger} \dot{G}_{k} .
$$

The evolution of $1-R^{2}$ is governed by

$$
\begin{aligned}
\frac{d R^{2}}{d t} & =2 \sum_{i=1}^{N}\left\{x_{k}^{\dagger} \dot{x}_{k}+x_{N+k}^{\dagger} \dot{x}_{N+k}\right\}=2 \sum_{i=1}^{N}\left\{x_{k}^{\dagger}\left[\theta x_{k}+x_{N+k}\right]+x_{N+k}^{\dagger}\left[\theta x_{N+k}+\left(1-R^{2}\right)^{3} G_{k}\right]\right\} \\
& =2 \theta R^{2}+2 h+2\left(1-R^{2}\right)^{3} L=2 \theta R^{2}-\left(1+R^{2}\right) \theta=-\left(1-R^{2}\right) \theta,
\end{aligned}
$$

which is more conveniently expressed

$$
\frac{d\left(1-R^{2}\right)}{d t}=\left(1-R^{2}\right) \theta
$$

We can integrate this equation formally via

$$
\begin{aligned}
\frac{d}{d t} \log \left(1-R^{2}\right) & =\frac{\frac{d}{d t}\left(1-R^{2}\right)}{1-R^{2}}=\theta \\
\frac{1-R^{2}}{1-R(0)^{2}} & =\exp \int_{0}^{t} \theta d s=\exp \left(-2 \int_{0}^{t} \frac{h+\left(1-R^{2}\right)^{3} L}{1+R^{2}} d s\right) .
\end{aligned}
$$

Lastly, starting with

$$
-\theta \frac{1+R^{2}}{2}=h+\left(1-R^{2}\right)^{3} L
$$

we differentiate both sides:

$$
-\frac{1+R^{2}}{2} \dot{\theta}-\theta \frac{1}{2} \frac{d R^{2}}{d t}=-\frac{1+R^{2}}{2} \dot{\theta}+\theta^{2} \frac{1-R^{2}}{2}=\dot{h}+3\left(1-R^{2}\right)^{3} \theta L+\left(1-R^{2}\right)^{3} \dot{L}
$$




$$
=2 \theta h+A+\left(1-R^{2}\right)^{3} K+3\left(1-R^{2}\right)^{3} \theta L+\left(1-R^{2}\right)^{3}\left[\theta L+\left(1-R^{2}\right)^{3} G^{2}+\sum_{k=1}^{N} x_{N+k}^{\dagger} \dot{G}_{k}\right]
$$

so that

$$
\dot{\theta}=-\frac{1-R^{2}}{1+R^{2}} \theta^{2}-\frac{4 \theta h+2 A}{1+R^{2}}-\frac{2\left(1-R^{2}\right)^{3}}{1+R^{2}}\left[4 \theta L+K+\sum_{k=1}^{N} x_{N+k}^{\dagger} \dot{G}_{k}\right]-\frac{2\left(1-R^{2}\right)^{6}}{1+R^{2}} G^{2}
$$

3.1.2. Including infinity. As in Chapter 2, the system (3.1.7) is well-defined on the non-collision points of the boundary sphere $\mathbb{S}^{6 N-1}$, so the system may be extended to those non-collision points of the boundary. If one or more of the bodies in the original problem escape to infinity (some $\left.\left\|q_{i}\right\| \rightarrow \infty\right)$ or one or more of the velocities become infinite (some $\left.\left\|\dot{q}_{i}\right\| \rightarrow \infty\right)$, then the compactification of $y=\left(q^{\dagger}, \dot{q}^{\dagger}\right)^{\dagger}$ is a trajectory $x$ that approaches the boundary. In addition, as will be seen in the next Section, there are trajectories on the boundary, which as compactifications of trajectories at $\infty$, represent new objects in Celestial Mechanics. We will explore the structure of solutions in the boundary and show how these solutions are relevant to real trajectories.

We will see that the boundary sphere is an interesting set. Even more so because in fact, all singularities of Celestial Mechanics are experienced on the boundary:

LEMMA 23. If a trajectory $q$ experiences a singularity at $t=\sigma<\infty$, then $x$ approaches the boundary as $t \rightarrow \sigma^{-}$. Moreover, if $q$ experiences a singularity at $\sigma$, then $\left(1-R^{2}\right)^{3} L \rightarrow \infty$ at least as fast as $\frac{1}{\sigma-t}$.

Proof. If there is a singularity at $t=\sigma$, then we must have $U \rightarrow \infty$ as $t \rightarrow \sigma^{-}$. By the conservation of energy, $T \rightarrow \infty$, as well, so

$$
\sum_{i=1}^{N} m_{i} \dot{q}_{i}^{\dagger} \dot{q}_{i}=\sum_{i=1}^{N} \frac{m_{i} x_{N+i}^{\dagger} x_{N+i}}{\left(1-R^{2}\right)^{2}} \rightarrow \infty
$$

Since $\left\|x_{N+i}\right\| \leq 1$, we must have that $1-R^{2} \rightarrow 0$, which means $x$ tends to the boundary.

From the integral formula (3.1.14), $1-R^{2} \rightarrow 0$ requires the exponential

$$
\exp \left(-2 \int_{0}^{\sigma} \frac{h+\left(1-R^{2}\right)^{3} L}{1+R^{2}} d s\right)=0
$$

which means

$$
\int_{0}^{\sigma} \frac{h+\left(1-R^{2}\right)^{3} L}{1+R^{2}} d s=\infty
$$

and since obviously $|h| \leq 1$, this is infinite only if $\left(1-R^{2}\right)^{3} L \rightarrow \infty$ at least as fast as $\frac{1}{\sigma-t}$, as $t \rightarrow \sigma^{-}$.

In terms of the uncompactified trajectory $q$, the first part of the Lemma says that a singularity $(\sigma<\infty)$ requires some particle's distance $q_{i}$ and/or velocity $\dot{q}_{i}$ must become unbounded in finite time.

By the Schwarz inequality,

$$
h^{2}=\left(\sum_{i=1}^{N} x_{N+i}^{\dagger} x_{i}\right)^{2} \leq \sum_{i=1}^{N}\left\|x_{N+i}\right\|^{2} \sum_{i=1}^{N}\left\|x_{i}\right\|^{2}=A^{2}\left(R^{2}-A^{2}\right),
$$

where $A^{2}=\sum_{i=1}^{N}\left\|x_{N+i}\right\|^{2}$, and the symmetry reduces the problem to one variable. It is easy to find the maximum of the right-hand side by taking the derivative and finding its zero, which is where $A=\frac{1}{\sqrt{2}} R$, which would make the right hand side equal $\frac{1}{4} R^{2}$. So, in fact $|h| \leq 1 / 2$.

Let us consider the possibilities for a trajectory $x$ that approaches the boundary as $t \rightarrow \sigma^{-}$, starting with the conservation of energy

$$
\frac{1}{2} \sum_{i=1}^{N} m_{i} \dot{q}_{i}^{\dagger} \dot{q}_{i}-\sum_{1 \leq j<k \leq N} \frac{m_{j} m_{k}}{\left\|q_{j}-q_{k}\right\|}=E
$$

(where $E$ is constant), expressed in terms of $x$ 


$$
\frac{1}{2} \sum_{i=1}^{N} \frac{m_{i} x_{N+i}^{\dagger} x_{N+i}}{\left(1-R^{2}\right)^{2}}-\sum_{1 \leq j<k \leq N} \frac{\left(1-R^{2}\right) m_{j} m_{k}}{\left\|x_{j}-x_{k}\right\|}=E .
$$

The two terms on the left of (3.1.15) must be finite or infinite together. If they are both bounded, then all the $\left\|x_{N+i}\right\|=O\left(1-R^{2}\right)$, and the minimum particle separation is bounded away from 0 , and since the limit point $x(\sigma) \notin \Delta$, we must have $\sigma=\infty$. The trajectory approaches a point in the boundary of the form

$$
p=\left(\begin{array}{c}
x^{*} \\
0_{3 N}
\end{array}\right)
$$

which will be seen in the sequel to be a critical point. Here $x^{*}$ is composed of the distinct 3 -vectors $x_{1}^{*}, \ldots x_{N}^{*}$. As shown in Section 4.3, this corresponds under compactification to a solution $q=a t+o(t)$ of (1.0.1) of particles escaping to infinity. In the terminology of Section 1.2, an escape trajectory $q=a t+o(t)$ diverges to infinity in the direction $x^{*}=\frac{a}{\|a\|}$. This case illustrates that the converse of the first part of Lemma 23 is not true; $x$ tending to the boundary is necessary but not sufficient to insure that $q$ has a singularity.

For example, the compactification of hyperbolic escape in the two-body problem, assuming the form of solutions to be proven in Chapter 5:

$$
\begin{aligned}
& y_{1}=q_{1}=a_{1} t+b_{1} \log t+c_{1}+o(1), \quad y_{2}=q_{2}=-\frac{m_{1}}{m_{2}} y_{1} \\
& y_{3}=\dot{q}_{1}=a_{1}+b_{1} \frac{1}{t}+o\left(\frac{1}{t}\right), \quad y_{4}=\dot{q}_{2}=-\frac{m_{1}}{m_{2}} y_{3},
\end{aligned}
$$

in center of mass coordinates. Since the two-body problem is planar, we may assume the motion takes place in $\mathbb{R}^{2}$. Let $M=m_{1}+m_{2}$. Then

$a_{2}=-\frac{m_{1}}{m_{2}} a_{1}, A=\left\|a_{1}\right\|^{2}+\left\|a_{2}\right\|^{2}=\left(1+\frac{m_{1}^{2}}{m_{2}^{2}}\right)\left\|a_{1}\right\|^{2} \quad a_{2}-a_{1}=-\frac{M}{m_{2}} a_{1}, \quad A_{12}=\left\|a_{2}-a_{1}\right\|=\frac{M}{m_{2}}\left\|a_{1}\right\|$.

We need the sum of the squares of the magnitudes of the $y_{i}$ :

$$
\begin{gathered}
r^{2}=\left(1+\frac{m_{1}^{2}}{m_{2}^{2}}\right)\left\{\left\|a_{1} t+b_{1} \log t+c_{1}+o(1)\right\|^{2}+\left\|a_{1}+b_{1} \frac{1}{t}+o\left(\frac{1}{t}\right)\right\|^{2}\right\} \\
=\left(1+\frac{m_{1}^{2}}{m_{2}^{2}}\right)\left\{\left\|a_{1}\right\|^{2} t^{2}+2 a_{1}^{\dagger} b_{1} t \log t+2 a_{1}^{\dagger} c_{1} t+o(t)\right\} \\
=\left(1+\frac{m_{1}^{2}}{m_{2}^{2}}\right)\left\|a_{1}\right\|^{2} t^{2}\left\{1+\frac{2 a_{1}^{\dagger} b_{1}}{\left\|a_{1}\right\|^{2}} \frac{\log t}{t}+\frac{2 a_{1}^{\dagger} c_{1}}{\left\|a_{1}\right\|^{2}} \frac{1}{t}+o\left(\frac{1}{t}\right)\right\} .
\end{gathered}
$$

From the formula

$$
b_{1}=\frac{M}{A_{12}^{3}} a_{1}, \text { so } \frac{a_{1}^{\dagger} b_{1}}{\left\|a_{1}\right\|^{2}}=\frac{M}{A_{12}^{3}}
$$

so we have

$$
r^{2}=A t^{2}\left\{1+\frac{2 M}{A_{12}^{3}} \frac{\log t}{t}+\frac{2 a_{1}^{\dagger} c_{1}}{\left\|a_{1}\right\|^{2}} \frac{1}{t}+o\left(\frac{1}{t}\right)\right\}
$$

and

$$
\frac{2}{1+\sqrt{1+4 r^{2}}}=\frac{1}{\sqrt{A} t}\left\{1-\frac{M}{A_{12}^{3}} \frac{\log t}{t}-\left(\frac{a_{1}^{\dagger} c_{1}}{\left\|a_{1}\right\|^{2}}+\frac{1}{2 \sqrt{A}}\right) \frac{1}{t}+o\left(\frac{1}{t}\right)\right\} .
$$


Now the compactified trajectory $x$ to low order

$$
\begin{aligned}
x_{1} & =\frac{1}{\sqrt{A} t}\left\{a_{1} t-\frac{M}{A_{12}^{3}} a_{1} \log t-\left(\frac{a_{1}^{\dagger} c_{1}}{\left\|a_{1}\right\|^{2}}+\frac{1}{2 \sqrt{A}}\right) a_{1}+b_{1} \log t+c_{1}+o(1)\right\} \\
& =\frac{1}{\sqrt{A}} a_{1}+\frac{1}{\sqrt{A}}\left[c_{1}-\left(\frac{a_{1}^{\dagger} c_{1}}{\left\|a_{1}\right\|^{2}}+\frac{1}{2 \sqrt{A}}\right) a_{1}\right] \frac{1}{t}+o\left(\frac{1}{t}\right) \\
x_{3} & =\frac{1}{\sqrt{A} t}\left\{a_{1}+b_{1} \frac{1}{t}-\frac{M}{A_{12}^{3}} a_{1} \frac{\log t}{t}-\left(\frac{a_{1}^{\dagger} c_{1}}{\left\|a_{1}\right\|^{2}}+\frac{1}{2 \sqrt{A}}\right) a_{1} \frac{1}{t}\right\}+o\left(\frac{1}{t^{2}}\right) \\
& =\frac{1}{\sqrt{A}} a_{1} \frac{1}{t}-\frac{M}{A_{12}^{3} \sqrt{A}} a_{1} \frac{\log t}{t^{2}}+\frac{M}{A_{12}^{3} \sqrt{A}} a_{1}-\frac{1}{\sqrt{A}}\left(\frac{a_{1}^{\dagger} c_{1}}{\left\|a_{1}\right\|^{2}}+\frac{1}{2 \sqrt{A}}\right) a_{1} \frac{1}{t^{2}}+o\left(\frac{1}{t^{2}}\right),
\end{aligned}
$$

and $x_{2}$ and $x_{4}$ are $-\frac{m_{1}}{m_{2}}$ times $x_{1}$ and $x_{3}$, respectively. It is easy to see that the trajectory in the ball approaches the boundary point

$$
p=\left(\begin{array}{c}
x^{*} \\
0_{4}
\end{array}\right), \quad x^{*}=\left(\begin{array}{c}
\frac{1}{\sqrt{A}} a_{1} \\
-\frac{m_{1}}{m_{2}} \frac{1}{\sqrt{A}} a_{1}
\end{array}\right) .
$$

Note $x^{*}$ is the unit 8 -vector in the direction $\left(\begin{array}{l}a_{1} \\ a_{2}\end{array}\right)$.

On the other hand, if both terms on the left of (3.1.15) are unbounded, then $1-R^{2}=o\left(\left\|x_{N+i}\right\|\right)^{1}$ for some $i$, and $\min \left\|x_{j}-x_{k}\right\|=o\left(1-R^{2}\right)$, which means $x$ and $q$ must approach $\Delta$. For example, a collision trajectory in the 2-body problem must be linear, so we can assume the particle 1 is on the positive $x$-axis, and particle 2 is on the negative $x$-axis. and we know from Pollard's text [87] that the separation

$$
q_{2}-q_{1} \sim c(\sigma-t)^{2 / 3}, \quad c^{3}=\frac{9 M}{2}
$$

in center of mass coordinates. So

$$
\begin{aligned}
& y_{1}=q_{1}=-\frac{m_{2}}{M} c(\sigma-t)^{2 / 3}+o\left((\sigma-t)^{2 / 3}\right), \quad y_{2}=q_{2}=-\frac{m_{1}}{m_{2}} y_{1} \\
& y_{3}=\dot{q}_{1}=\frac{2}{3} \frac{m_{2}}{M} c(\sigma-t)^{-1 / 3}+o\left((\sigma-t)^{-1 / 3}\right), \quad y_{4}=\dot{q}_{2}=-\frac{m_{1}}{m_{2}} y_{3} .
\end{aligned}
$$

The magnitude $r$ is dominated by $y_{3}$ and $y_{4}$

$$
\begin{gathered}
r^{2}=\frac{4}{9} \frac{m_{1}^{2}+m_{2}^{2}}{M^{2}} c^{2}(\sigma-t)^{-2 / 3}+o\left((\sigma-t)^{-2 / 3}\right) \\
\frac{2}{1+\sqrt{1+4 r^{2}}}=\frac{3}{2} \frac{M}{\sqrt{m_{1}^{2}+m_{2}^{2}}} \frac{1}{c}(\sigma-t)^{1 / 3}\left[1-\frac{3}{4} \frac{M}{\sqrt{m_{1}^{2}+m_{2}^{2}}} \frac{1}{c}(\sigma-t)^{1 / 3}\right]+o\left((\sigma-t)^{2 / 3}\right) .
\end{gathered}
$$

Now the compactified trajectory $x$ to low order

$$
\begin{aligned}
& x_{1}=-\frac{3}{2} \frac{m_{2}}{\sqrt{m_{1}^{2}+m_{2}^{2}}}(\sigma-t)+\frac{9}{8} \frac{m_{2} M}{m_{1}^{2}+m_{2}^{2}} \frac{1}{c}(\sigma-t)^{4 / 3}+o\left((\sigma-t)^{2 / 3}\right) \\
& x_{3}=-\frac{m_{2}}{\sqrt{m_{1}^{2}+m_{2}^{2}}}+\frac{3}{4} \frac{m_{2} M}{m_{1}^{2}+m_{2}^{2}} \frac{1}{c}(\sigma-t)^{1 / 3}+o\left((\sigma-t)^{1 / 3}\right),
\end{aligned}
$$

and $x_{2}$ and $x_{4}$ are $-\frac{m_{1}}{m_{2}}$ times $x_{1}$ and $x_{3}$, respectively. It is easy to see that the trajectory in the ball approaches the boundary point

$$
p=\left(\begin{array}{c}
0 \\
0 \\
-\frac{m_{2}}{\sqrt{m_{1}^{2}+m_{2}^{2}}} \\
\frac{m_{1}}{\sqrt{m_{1}^{2}+m_{2}^{2}}}
\end{array}\right) .
$$

a unit 4-vector.

\footnotetext{
${ }^{1}$ That means $\left\|x_{N+i}\right\|$ either doesn't tend to 0 or at least decays more slowly than any multiple of $1-R^{2}$.
} 
We know from Lemma 23 that the quantity $L$ is unbounded near $\Delta$. We can use conservation of energy to constrain the growth of $L$ near a singularity; first, multiply (3.1.15) by $\left(1-R^{2}\right)^{2}$ and rearrange

$$
\left(1-R^{2}\right)^{3} \sum_{1 \leq j<k \leq N} \frac{m_{j} m_{k}}{\left\|x_{j}-x_{k}\right\|}=\frac{1}{2} \sum_{i=1}^{N} m_{i} x_{N+i}^{\dagger} x_{N+i}-E\left(1-R^{2}\right)^{2} .
$$

Since every $\left\|x_{N+i}\right\| \leq 1$, the first term on the right is bounded by $\frac{1}{2} \sum_{i=1}^{N} m_{i}$, we have the bound:

$$
0 \leq\left(1-R^{2}\right)^{3} \sum_{1 \leq j<k \leq N} \frac{m^{2}}{g_{j k}} \leq\left(1-R^{2}\right)^{3} \sum_{1 \leq j<k \leq N} \frac{m_{j} m_{k}}{g_{j k}} \leq D:=\frac{1}{2} \sum_{i=1}^{N} m_{i}-E\left(1-R^{2}\right)^{2},
$$

where $m$ is the minimum of the $m_{i}$. In particular, we must have $D \geq 0$, so we have the bound

$$
0 \leq\left(1-R^{2}\right)^{3} \sum_{1 \leq j<k \leq N} \frac{1}{g_{j k}} \leq \frac{D}{m^{2}} .
$$

Also let $M$ be the maximum of the $m_{i}$, so that we can express a bound for $G_{k}$ :

$$
\left\|G_{k}\right\| \leq \sum_{j \neq k} \frac{m_{i}\left\|x_{j}-x_{k}\right\|}{g_{j k}^{3}} \leq 2 M \sum_{j \neq k} \frac{1}{g_{j k}^{2}} .
$$

Then

$$
|L| \leq \sum_{k=1}^{N}\left\|x_{N+k}\right\|\left\|G_{k}\right\| \leq 2 M N \sum_{j \neq k} \frac{1}{g_{j k}^{2}} \leq 4 M N \sum_{1 \leq j<k \leq N} \frac{1}{g_{j k}^{2}} \leq 4 M N\left(\sum_{j<k} \frac{1}{g_{j k}}\right)^{2},
$$

where the second-last inequality is because $\sum_{j \neq k} \frac{1}{g_{j k}^{2}}$ counts each $\frac{1}{g_{j k}^{2}}$ twice and $\sum_{1 \leq j<k \leq N} \frac{1}{g_{j k}^{2}}$ counts them once, and the last inequality follows from the Schwarz inequality. We have the bound for $\left(1-R^{2}\right)^{6} L$

$$
\left(1-R^{2}\right)^{6}|L| \leq 4 M N\left(1-R^{2}\right)^{6}\left(\sum_{1 \leq j<k \leq N} \frac{1}{g_{j k}}\right)^{2} \leq 4 M N\left(\left(1-R^{2}\right)^{3} \sum_{1 \leq j<k \leq N} \frac{1}{g_{j k}}\right)^{2} \leq \frac{4 M N D^{2}}{m^{4}} .
$$

It also follows that $\left(1-R^{2}\right)^{6+\epsilon} L$ approaches 0 as $x$ approaches the boundary and is identically 0 on the boundary, for any $\epsilon>0$.

3.1.3. Seeking a series expansion. Now let us suppose that a solution to (3.1.7), starting in the interior, exists for all time and approaches a critical point $x^{*}$ in the boundary, and we will seek a series approximation in $1 / t$ near the critical point, starting with

$$
x=\left(\begin{array}{c}
x^{*}+a_{1} / t+a_{2} / t^{2}+a_{3} / t^{3}+\ldots \\
b_{1} / t+b_{2} / t^{2}+b_{3} / t^{3}+\ldots
\end{array}\right), \quad \dot{x}=\left(\begin{array}{c}
-a_{1} / t^{2}-2 a_{2} / t^{3}+\ldots \\
-b_{1} / t^{2}-2 b_{2} / t^{3}-3 b_{3} / t^{4}+\ldots
\end{array}\right)
$$

where the $a_{k}$ and $b_{k}$ are column $3 N$-vectors. In fact, we do not expect analytic solutions $x$; however, in the sequel we shall define and develop approximating systems to (3.1.1), which we will see can be solved in closed form and have analytic solutions. The limited results we obtain here will be valid for the approximating systems.

For each positive integer $m$, let

$$
A_{m}=a_{m}^{\dagger} x^{*} \text { and } B_{m}=b_{m}^{\dagger} x^{*} .
$$

To compute the right hand side of (3.1.7) through $1 / t^{3}$, we shall need

$$
\begin{aligned}
h=\sum_{i=1}^{N} x_{N+i}^{\dagger} x_{i} & =\frac{b_{1}^{\dagger} x^{*}}{t}+\frac{b_{1}^{\dagger} a_{1}+b_{2}^{\dagger} x^{*}}{t^{2}}+\frac{b_{1}^{\dagger} a_{2}+b_{2}^{\dagger} a_{1}+b_{3}^{\dagger} x^{*}}{t^{3}}+O\left(\frac{1}{t^{4}}\right) \\
& =\frac{B_{1}}{t}+\frac{b_{1}^{\dagger} a_{1}+B_{2}}{t^{2}}+\frac{b_{1}^{\dagger} a_{2}+b_{2}^{\dagger} a_{1}+B_{3}}{t^{3}}+O\left(\frac{1}{t^{4}}\right) .
\end{aligned}
$$


Next

$1-R^{2}=1-\left\{x^{* \dagger} x^{*}+2 \frac{a_{1}^{\dagger} x^{*}}{t}+\frac{b_{1}^{\dagger} b_{1}+a_{1}^{\dagger} a_{1}+2 a_{2}^{\dagger} x^{*}}{t^{2}}+O\left(\frac{1}{t^{3}}\right)\right\}=-\frac{2 A_{1}}{t}-\frac{b_{1}^{\dagger} b_{1}+a_{1}^{\dagger} a_{1}+2 A_{2}}{t^{2}}+O\left(\frac{1}{t^{3}}\right)$,

since $x^{* \dagger} x^{*}=1$. Now since $R^{2} \leq 1$, we have the convergent series expansion

$$
\frac{2}{1+R^{2}}=\frac{1}{1-\frac{1-R^{2}}{2}}=\sum_{m=0}^{\infty}\left(\frac{1-R^{2}}{2}\right)^{m}
$$

valid for all $R \in[0,1]$, so we can approximate

$$
\begin{gathered}
\frac{2}{1+R^{2}}=1+\frac{1-R^{2}}{2}+\left(\frac{1-R^{2}}{2}\right)^{2}+O\left(\left(1-R^{2}\right)^{3}\right)=1-\frac{A_{1}}{t}-\frac{b_{1}^{\dagger} b_{1}+a_{1}^{\dagger} a_{1}+2 A_{2}}{2 t^{2}}+\frac{4 A_{1}^{2}}{4 t^{2}}+O\left(\frac{1}{t^{3}}\right) \\
=1-\frac{A_{1}}{t}-\frac{b_{1}^{\dagger} b_{1}+a_{1}^{\dagger} a_{1}+2 A_{2}-2 A_{1}^{2}}{2 t^{2}}+O\left(\frac{1}{t^{3}}\right),
\end{gathered}
$$

where we've replaced $O\left(\left(1-R^{2}\right)^{3}\right)$ with $O\left(\frac{1}{t^{3}}\right)$ due to (3.1.18). and we will not need the omitted $1 / t^{3}$ terms for this calculation. Next

$$
\begin{gathered}
\theta=-\frac{2}{1+R^{2}}\left(h+\left(1-R^{2}\right)^{3} L\right) \\
=-\left(1-\frac{A_{1}}{t}-\frac{b_{1}^{\dagger} b_{1}+a_{1}^{\dagger} a_{1}+2 A_{2}-2 A_{1}^{2}}{2 t^{2}}+O\left(\frac{1}{t^{3}}\right)\right)\left(\frac{B_{1}}{t}+\frac{b_{1}^{\dagger} a_{1}+B_{2}}{t^{2}}+\frac{b_{1}^{\dagger} a_{2}+b_{2}^{\dagger} a_{1}+B_{3}}{t^{3}}+O\left(\frac{1}{t^{4}}\right)\right),
\end{gathered}
$$

because $\left(1-R^{2}\right)^{3} L=O\left(\frac{1}{t^{4}}\right)$. So

$$
\theta=-\frac{B_{1}}{t}+\frac{B_{1} A_{1}-b_{1}^{\dagger} a_{1}-B_{2}}{t^{2}}+\frac{\theta_{3}}{t^{3}}+O\left(\frac{1}{t^{4}}\right)
$$

where

$$
\theta_{3}=-\left[b_{1}^{\dagger} a_{2}+b_{2}^{\dagger} a_{1}+B_{3}\right]+\left(b_{1}^{\dagger} a_{1}+B_{2}\right) A_{1}+\left(\frac{1}{2} b_{1}^{\dagger} b_{1}+\frac{1}{2} a_{1}^{\dagger} a_{1}+A_{2}-A_{1}^{2}\right) B_{1} .
$$

Next we express (3.1.7) in terms of powers of $1 / t$ and equate terms of like power

$$
\begin{aligned}
& -\sum_{r=2}^{\infty} \frac{(r-1) a_{r-1}}{t^{r}}=\left(-\frac{B_{1}}{t}+\frac{B_{1} A_{1}-b_{1}^{\dagger} a_{1}-B_{2}}{t^{2}}+\frac{\theta_{3}}{t^{3}}\right) \sum_{r=0}^{\infty} \frac{a_{r}}{t^{r}}+\sum_{r=1}^{\infty} \frac{b_{r}}{t^{r}} \\
& -\sum_{r=2}^{\infty} \frac{(r-1) b_{r-1}}{t^{r}}=\left(-\frac{B_{1}}{t}+\frac{B_{1} A_{1}-b_{1}^{\dagger} a_{1}-B_{2}}{t^{2}}+\frac{\theta_{3}}{t^{3}}\right) \sum_{r=1}^{\infty} \frac{b_{r}}{t^{r}}-\frac{8 A_{1}^{3}}{t^{3}} G,
\end{aligned}
$$


which we can rearrange a little bit

$$
\begin{aligned}
& \sum_{r=2}^{\infty} \frac{(r-1) a_{r-1}}{t^{r}}=B_{1} \sum_{r=1}^{\infty} \frac{a_{r-1}}{t^{r}}+\left(b_{1}^{\dagger} a_{1}+B_{2}-A_{1} B_{1}\right) \sum_{r=2}^{\infty} \frac{a_{r-2}}{t^{r}}-\theta_{3} \sum_{r=3}^{\infty} \frac{a_{r-3}}{t^{r}}-\sum_{r=1}^{\infty} \frac{b_{r}}{t^{r}} \\
& =\frac{B_{1} x^{*}-b_{1}}{t}+\frac{B_{1} a_{1}+\left(b_{1}^{\dagger} a_{1}+B_{2}-A_{1} B_{1}\right) x^{*}-b_{2}}{t^{2}} \\
& +\sum_{r=3}^{\infty} \frac{B_{1} a_{r-1}+\left(b_{1}^{\dagger} a_{1}+B_{2}-A_{1} B_{1}\right) a_{r-2}-\theta_{3} a_{r-3}-b_{r}}{t^{r}} \\
& \sum_{r=2}^{\infty} \frac{(r-1) b_{r-1}}{t^{r}}=B_{1} \sum_{r=2}^{\infty} \frac{b_{r-1}}{t^{r}}+\left(b_{1}^{\dagger} a_{1}+B_{2}-A_{1} B_{1}\right) \sum_{r=3}^{\infty} \frac{b_{r-2}}{t^{r}}-\theta_{3} \sum_{r=4}^{\infty} \frac{b_{r-3}}{t^{r}}+\left(\frac{8 A_{1}^{3}}{t^{3}}+O\left(\frac{1}{t^{4}}\right)\right) G \\
& =\frac{B_{1} b_{1}}{t^{2}}+\frac{B_{1} b_{2}+\left(b_{1}^{\dagger} a_{1}+B_{2}-A_{1} B_{1}\right) b_{1}+8 A_{1}^{3} G}{t^{3}} \\
& +\sum_{r=4}^{\infty} \frac{B_{1} b_{r-1}+\left(b_{1}^{\dagger} a_{1}+B_{2}-A_{1} B_{1}\right) b_{r-2}-\theta_{3} b_{r-3}}{t^{r}}
\end{aligned}
$$

Our next step is to set corresponding coefficients equal, which yields algebraic equations. The $r=1$ equation for $\dot{x}_{i}$ gives $b_{1}=B_{1} x^{*}$, and the $r=2$ equation for $\dot{x}_{N+i}$ give that $B_{1}=1$. So $b_{1}=x^{*}$. The $r=2$ equation for $\dot{x}_{i}$ is easy to solve:

$$
\begin{gathered}
a_{1}=B_{1} a_{1}+\left(b_{1}^{\dagger} a_{1}+B_{2}-A_{1} B_{1}\right) x^{*}-b_{2} \\
0=\left(x^{*} a_{1}+B_{2}-A_{1}\right) x^{*}-b_{2} \\
b_{2}=B_{2} x^{*}
\end{gathered}
$$

With this information, we can simplify

$$
\theta=-\frac{1}{t}-\frac{B_{2}}{t^{2}}+\frac{\theta_{3}}{t^{3}}+O\left(\frac{1}{t^{4}}\right)
$$

and

$$
\begin{gathered}
\theta_{3}=-\left[x^{* \dagger} a_{2}+B_{2} x^{* \dagger} a_{1}+B_{3}\right]+\left(x^{* \dagger} a_{1}+B_{2}\right) A_{1}+\frac{1}{2} x^{* \dagger} x^{*}+\frac{1}{2} a_{1}^{\dagger} a_{1}+A_{2}-A_{1}^{2} \\
=-\left[A_{2}+A_{1} B_{2}+B_{3}\right]+\left(A_{1}+B_{2}\right) A_{1}+\frac{1}{2}+\frac{1}{2} a_{1}^{\dagger} a_{1}+A_{2}-A_{1}^{2} \\
=-B_{3}+\frac{1}{2}+\frac{1}{2} a_{1}^{\dagger} a_{1}
\end{gathered}
$$

The $r=3$ equation for $\dot{x}_{N+i}$ requires an expansion of $G_{i}$, which we will not attempt. The $1 / t^{3}$ equation for $\dot{x}_{i}$

simplifies to

$$
2 a_{2}=B_{1} a_{2}+\left(b_{1}^{\dagger} a_{1}+B_{2}-A_{1} B_{1}\right) a_{1}-\theta_{3} x^{*}-b_{3},
$$

$$
a_{2}=\left(x^{*} a_{1}+B_{2}-A_{1}\right) a_{1}-\theta_{3} x^{*}-b_{3},
$$

using the fact that $b_{1}=x^{*}$. Substituting for $\theta_{3}$ yields

$$
a_{2}=B_{2} a_{1}-\theta_{3} x^{*}-b_{3}=B_{2} a_{1}-\left(-B_{3}+\frac{1}{2}+\frac{1}{2} a_{1}^{\dagger} a_{1}\right) x^{*}-b_{3},
$$

which we can re-arrange:

$$
b_{3}-B_{3} x^{*}=B_{2} a_{1}-a_{2}-\left[\frac{1}{2} a_{1}^{\dagger} a_{1}+\frac{1}{2}\right] x^{*} .
$$

Next we apply $x^{* \dagger}$ to both sides yielding $\frac{1}{2} a_{1 j}^{\dagger} a_{1 j}+\frac{1}{2}=A_{1} B_{2}-A_{2}$, which we can use to simplify the previous equation:

$$
b_{3}-B_{3} x^{*}=B_{2}\left(a_{1}-A_{1} x^{*}\right)-\left(a_{2}-A_{2} x^{*}\right),
$$

which relates the components perpendicular to $x^{*}$ of some of the coefficients. And if $b_{3}=B_{3} x^{*}$, then in particular, the component of $a_{2}$ perpendicular to $x^{*}$ is proportional to the component of $a_{1}$ perpendicular 
to $x^{*}$. Going beyond these terms in the expansion requires expanding $G_{i}$ and $L$, and is significantly more difficult. All we can conclude is that if there is a series expansion in $1 / t$ for a trajectory $x$ approaching a critical point $\left(x^{* \dagger}, 0_{3 N}^{\dagger}\right)^{\dagger}$ in the boundary, then $b_{1}=x^{*}$, and $b_{2}=B_{2} x^{*}$.

\subsection{The System on the Boundary Sphere}

In this Section we study the system restricted to the boundary, deriving its critical points and all solutions explicitly. We compute the Jacobian at the critical points (none of which are isolated) and find the eigenvalues are all 0 . Thus, the Jacobian does not indicate anything about stability properties of the critical points. We find analytic solutions in the boundary and give leading terms in $1 / t$ of boundary trajectories, exhibiting their asymptotic behavior. We also study the Jacobian along a trajectory in the boundary and note that its eigenvalues are negative (and tend to 0 ).

From (3.1.13) it appears that the boundary would be an invariant set, and we will see that as long as there is no collision, a trajectory starting on the boundary stays on the boundary. However, as particles $i$ and $j$ approach a collision, $L=O\left(1 / g_{i j}^{2}\right)$, and for $n=1,2,3,4,5$, the quantity $\left(1-R^{2}\right)^{n} L$ restricted to the boundary is similar to a Dirac $\delta$-function: vanishing except at collisions, where it is infinite. We cannot say that $\dot{R}=0$ on the boundary, so the boundary is not an invariant set. It is possible that a trajectory in the open ball could in finite time reach a point of collision on the boundary. For the uncompactified system, such a trajectory corresponds to two or more particles escaping to $\infty$ while approaching each other, for example the solution of Xia [117].

Even though the boundary is not invariant, it will prove useful to study the system on it. Setting $R=1$ in (3.1.7), yields the equations of motion on the open set $\mathbb{S}^{6 N-1} \backslash \Delta$, the non-collision points of the boundary:

$$
\begin{aligned}
\dot{x}_{i} & =-h x_{i}+x_{N+i} \\
\dot{x}_{N+i} & =-h x_{N+i} .
\end{aligned}
$$

In fact, these equations are not singular on $\Delta$ and could be extended to all of the boundary at the potential cost of diminishing relevance to real trajectories near collisions. It is easy to see that the critical points are where all $x_{N+i}=0_{3}$, which correspond to (uncompactified) particles reaching infinity with finite velocity. These points comprise a $3 N-1$-sphere $\left\{x \mid \sum x_{i}^{\dagger} x_{i}=1, x_{N+i}=0_{3}\right\}$ within the boundary $6 N$ - 1-sphere. Since the full compactified system (3.1.7) has no interior critical points, this $3 N-1$-sphere is also the critical point set for (3.1.7).

Returning to (3.1.7), we can compute its Jacobian at a point on the boundary away from $\Delta$. The terms cubic in $1-R^{2}$ can be ignored, because if $N$ is either $G_{i}$ or $L$; then the partial derivative with respect to $x_{i}$ or $x_{N+i}$

$$
\frac{\partial\left(1-R^{2}\right)^{3} N}{\partial x}=3\left(1-R^{2}\right)^{2} \frac{\partial\left(1-R^{2}\right)}{\partial x} N+\left(1-R^{2}\right)^{3} \frac{\partial N}{\partial x},
$$

which vanishes on the boundary. So the Jacobian of (3.1.7) at a point on the boundary away from $\Delta$ is the same as the Jacobian of

$$
\begin{aligned}
\dot{x}_{i} & =-\frac{2}{1+R^{2}} h x_{i}+x_{N+i} \\
\dot{x}_{N+i} & =-\frac{2}{1+R^{2}} h x_{N+i} .
\end{aligned}
$$

We need the following:

$$
\nabla_{x} R^{2}=2 x, \quad \nabla_{x} \frac{2}{1+R^{2}}=-x, \quad \nabla_{x} h=\left(\begin{array}{cc}
O_{3 N} & I_{3 N} \\
I_{3 N} & O_{3 N}
\end{array}\right) x .
$$

Then we have the Jacobian on the boundary:

$$
\left.J a c\right|_{\partial}=h x x^{\dagger}-x x^{\dagger}\left(\begin{array}{cc}
O_{3 N} & I_{3 N} \\
I_{3 N} & O_{3 N}
\end{array}\right)+\left(\begin{array}{cc}
-h I_{3 N} & I_{3 N} \\
0 & -h I_{3 N}
\end{array}\right),
$$


where $x x^{\dagger}$ is a $6 N$-square matrix. At the critical points, all $x_{N+j}=0$, so $h=0$, and the Jacobian reduces to

$$
\left(\begin{array}{cccccccc}
O_{3} & O_{3} & \ldots & O_{3} & I_{3}-x_{1} x_{1}^{\dagger} & -x_{1} x_{2}^{\dagger} & \ldots & -x_{1} x_{N}^{\dagger} \\
O_{3} & O_{3} & \ldots & O_{3} & -x_{2} x_{1}^{\dagger} & I_{3}-x_{2} x_{2}^{\dagger} & \ldots & -x_{2} x_{N}^{\dagger} \\
\vdots & \vdots & \ddots & \vdots & \vdots & \vdots & \ddots & \vdots \\
O_{3} & O_{3} & \ldots & O_{3} & -x_{N} x_{1}^{\dagger} & -x_{N} x_{2}^{\dagger} & \ldots & I_{3}-x_{N} x_{N}^{\dagger} \\
O_{3} & O_{3} & \ldots & O_{3} & O_{3} & O_{3} & \ldots & O_{3} \\
O_{3} & O_{3} & \ldots & O_{3} & O_{3} & O_{3} & \ldots & O_{3} \\
\vdots & \vdots & \ddots & \vdots & \vdots & \vdots & \ddots & \vdots \\
O_{3} & O_{3} & \ldots & O_{3} & O_{3} & O_{3} & \ldots & O_{3}
\end{array}\right),
$$

where each element of the matrix is a $3 \times 3$ block. The Jacobian is nilpotent, with eigenvalues all 0 . This gives us no information about trajectories attracted to the critical points, but as we will see next, the critical point set does attract trajectories in the boundary. Moreover, in the next Chapter, we will see that the critical point set and boundary trajectories approaching it also attract nearby (interior) trajectories.

Let us define $A=\sum_{i} x_{N+i}^{\dagger} x_{N+i}$. Obviously, we will have $A \in[0,1]$. Then from (3.1.10) and (3.1.11), or directly from (3.2.1), it is easy to see that in the boundary, $h$ and $A$ satisfy the two scalar equations:

$$
\begin{aligned}
\dot{A} & =-2 h A \\
\dot{h} & =A-2 h^{2},
\end{aligned}
$$

and the only critical point is at the origin of the $(h, A)$ plane. Considering the ratio, we have

$$
\frac{d}{d t} \frac{h}{A}=\frac{A-2 h^{2}}{A}+\frac{2 h^{2} A}{A^{2}}=1,
$$

and we can express (3.2.4) as

$$
\dot{A} / A=-2 h .
$$

The first is easy to integrate:

$$
h / A=h(0) / A(0)+t
$$

so

$$
\begin{aligned}
-\dot{A} / A^{2} & =2 \frac{h}{A}=2\left(\frac{h(0)}{A(0)}+t\right) \\
\frac{1}{A} & =\frac{1+2 h(0) t+A(0) t^{2}}{A(0)} \\
A & =\frac{A(0)}{1+2 h(0) t+A(0) t^{2}} .
\end{aligned}
$$

Substituting for $A$ in the $h / A$ equation yields $h$ in closed form:

$$
h=\frac{h(0)+A(0) t}{1+2 h(0) t+A(0) t^{2}} .
$$

Note that the quantity $\frac{h^{2}-A}{A^{2}}$ is a constant of the motion, so the motion is restricted to a conic section in the $(h, A)$ plane. For motion in the closed ball, the relations

$$
0 \leq\left\|x_{i} \pm x_{N+i}\right\|^{2}=\sum_{i=1}^{N}\left(x_{i} \pm x_{N+i}\right)^{\dagger}\left(x_{i} \pm x_{N+i}\right)=\sum_{i=1}^{N}\left(x_{i}^{\dagger} x_{i} \pm 2 x_{i}^{\dagger} x_{N+i}+x_{N+i}^{\dagger} x_{N+i}\right)=R^{2} \pm 2 h
$$

imply that $|h| \leq \frac{1}{2} R^{2} \leq \frac{1}{2}$. Note that along a trajectory on the boundary both $h$ and $A$ approach 0 as $t \rightarrow \infty$, but $h \sim \frac{1}{t}$, and $A \sim \frac{1}{t^{2}}$, so $h / A \sim t$ as $t \rightarrow \pm \infty$. 
Now let

then it is easy to see that

$$
E=\exp \left[-\int_{0}^{t} h(s) d s\right]
$$

$$
x=E\left(\begin{array}{cc}
I_{3 N} & t I_{3 N} \\
O_{3 N} & I_{3 N}
\end{array}\right) x(0)
$$

solves (3.2.1):

$$
\dot{E}=-h E,
$$

so

$$
\begin{aligned}
\dot{x} & =-h E\left(\begin{array}{cc}
I_{3 N} & t I_{3 N} \\
O_{3 N} & I_{3 N}
\end{array}\right) x(0)+E\left(\begin{array}{cc}
O_{3 N} & I_{3 N} \\
O_{3 N} & O_{3 N}
\end{array}\right) x(0) \\
& =-h x+\left(\begin{array}{cc}
O_{3 N} & I_{3 N} \\
O_{3 N} & O_{3 N}
\end{array}\right) x,
\end{aligned}
$$

which is (3.2.1). To compute $E$ in closed form, take the square of the magnitude of both sides of (3.2.8), yielding

$$
\begin{gathered}
1=\sum_{i=1}^{N}\left(x_{i}^{\dagger} x_{i}+x_{N+i}^{\dagger} x_{N+i}\right) \\
=E^{2} \sum_{i=1}^{N}\left(x_{i}^{\dagger}(0) x_{i}(0)+2 t x_{i}^{\dagger}(0) x_{N+i}(0)+t^{2} x_{N+i}^{\dagger}(0) x_{N+i}(0)+x_{N+i}^{\dagger}(0) x_{N+i}(0)\right) \\
=E^{2}\left(1+2 h(0) t+A(0) t^{2}\right),
\end{gathered}
$$

so

and we have

$$
E=\frac{1}{\sqrt{1+2 h(0) t+A(0) t^{2}}}
$$

$$
x_{i}=\frac{x_{i}(0)+x_{N+i}(0) t}{\sqrt{1+2 h(0) t+A(0) t^{2}}}, \quad x_{N+i}=\frac{x_{N+i}(0)}{\sqrt{1+2 h(0) t+A(0) t^{2}}}
$$

or

$$
x=\frac{1}{\sqrt{1+2 h(0) t+A(0) t^{2}}}\left(\begin{array}{cc}
I_{3 N} & t I_{3 N} \\
O_{3 N} & I_{3 N}
\end{array}\right) x(0) .
$$

This is an exact solution of the compactified flow on the boundary, and if the trajectory avoids collision, the trajectory approaches the critical point $\left(x_{N+i}(0)^{\dagger} / \sqrt{A(0)}, 0_{3 N}^{\dagger}\right) \dagger$ as $t \rightarrow \infty$.

This solution has $6 N$ parameters $x(0)$ constrained only by the ten integrals of the motion and being restricted to the boundary.

As noted above, the system (3.2.1) viewed independently of its derivation is not singular at points of collision, so it may be extended to the full boundary sphere, and (3.2.9) is the solution on the whole boundary. Starting anywhere on the boundary, the trajectory approaches a point $\left(x^{* \dagger}, 0_{3 N}^{\dagger}\right)^{\dagger}$ on the critical $3 N-1$ sphere $\left\{\sum_{i=1}^{N} x_{i}^{\dagger} x_{i}=1, \forall j \leq N, x_{N+j}=0\right\}$ in the boundary $6 N-1$ sphere. Moreover, each 3-vector $x_{N+i}$ is for all time parallel to $x_{N+i}(0)$. Every starting point $x(0)$ on the boundary sphere moves under the flow toward $\left(\frac{x_{N+i}(0)^{\dagger}}{\sqrt{A(0)}}, 0_{3 N}^{\dagger}\right)^{\dagger}=\left(x^{* \dagger}, 0_{3 N}^{\dagger}\right)^{\dagger}$ as $t \rightarrow \infty$. So given a critical point $\left(x^{* \dagger}, 0_{3 N}^{\dagger}\right)^{\dagger}$ on the boundary sphere, its stable manifold consists of the set of points

$$
\left\{\left(x_{i}(0), a x_{i}^{*}\right) \mid a \in[0,1], \sum x_{i}(0)^{\dagger} x_{i}(0)=1-a^{2}\right\} .
$$

For $a=0$, this is just the critical point itself, and for $a=1$, the point $\left(0, x^{*}\right)$.

As an aid to visualization, we consider a low-dimensional analog in Figure 3.2.1. Rather than the critical point set $\mathbb{S}^{3 N-1}$ within the boundary $\mathbb{S}^{6 N-1}$ for the $N$-body problem, we sketch some boundary trajectories 


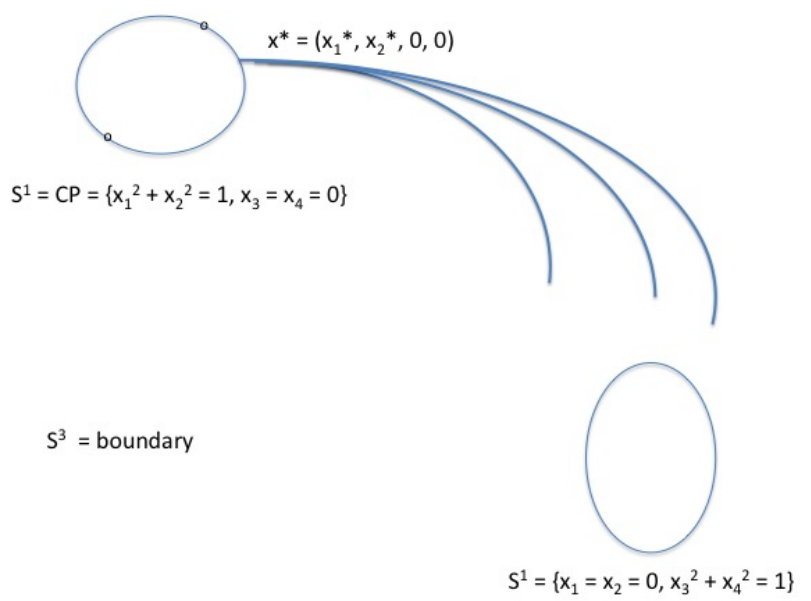

FiguRE 3.2.1. Boundary trajectories tending to the critical point set. The two singular points $\left\{x_{1}=x_{2}\right\}$ in the critical set $C P=\mathbb{S}^{1}$ are marked as o.

tending to the critical point set imagined as an $\mathbb{S}^{1}$ within the boundary $\mathbb{S}^{3}$. The third dimension is taken perpendicular to plane of the page, although there is actually no plane within $\mathbb{S}^{3}$ containing the two unlinked $\mathbb{S}^{1}$. Each critical point has a two-dimensional stable manifold in the boundary, which is suggested by the three trajectories illustrated.

Note that the solution is an analytic function for sufficiently large $t$, so it has a power series in $1 / t$, convergent on $(T, \infty]$ for some large $T$. For future reference, we evaluate the asymptotic behavior of this trajectory as $t \rightarrow \infty$, again, so long as there is no collision. We now consider trajectories in the boundary approaching the critical set and wish to expand in $1 / t$ a solution near a critical point $\left(x^{* \dagger}, 0_{3 N}^{\dagger}\right)^{\dagger}$. The denominator of (3.2.9) is the square root of

$$
A(0) t^{2}+2 h(0) t+1 \sim A(0) t^{2}\left(1+2 \frac{h(0)}{A(0) t}+\frac{1}{A(0) t^{2}}\right),
$$

so we expand the denominator using the expansion

$$
\frac{1}{\sqrt{1+r}}=1-\frac{1}{2} r+\frac{3}{8} r^{2}+O\left(r^{3}\right)
$$

as

$$
\begin{aligned}
& \frac{1}{\sqrt{A(0)} t}\left(1+\frac{2 h(0) t+1}{A(0) t^{2}}\right)^{-1 / 2}=\frac{1}{\sqrt{A(0)} t}\left(1-\frac{1}{2} \frac{2 h(0) t+1}{A(0) t^{2}}+\frac{3}{8}\left(\frac{2 h(0) t+1}{A(0) t^{2}}\right)^{2}\right)+O\left(\frac{1}{t^{4}}\right) \\
= & \frac{1}{\sqrt{A(0)}}\left(\frac{1}{t}-\frac{h(0)}{A(0)} \frac{1}{t^{2}}+\left(\frac{3}{2} \frac{h(0)^{2}}{A(0)^{2}}-\frac{1}{2 A(0)}\right) \frac{1}{t^{3}}\right)+O\left(\frac{1}{t^{4}}\right),
\end{aligned}
$$

and the asymptotic behavior can be computed

$$
\begin{aligned}
x_{i}(t) & =x_{i}^{*}+\left(\frac{x_{i}(0)}{\sqrt{A(0)}}-\frac{h(0)}{A(0)} x_{i}^{*}\right) \frac{1}{t}+\left(\frac{x_{i}(0)}{\sqrt{A(0)}}+\left(\frac{3}{2} \frac{h(0)^{2}}{A(0)^{2}}-\frac{1}{2 A(0)}\right) x_{i}^{*}\right) \frac{1}{t^{2}}+O\left(\frac{1}{t^{3}}\right) \\
x_{N+i}(t) & =\left(\frac{1}{t}-\frac{h(0)}{A(0) t^{2}}+\left(\frac{3}{2} \frac{h(0)^{2}}{A(0)^{2}}-\frac{1}{2 A(0)}\right) \frac{1}{t^{3}}\right) x_{i}^{*}+O\left(\frac{1}{t^{4}}\right) \\
10) \quad h & =\frac{1}{t}-\frac{h(0)}{A(0) t^{2}}+\left(3 \frac{h(0)^{2}}{A(0)^{2}}+\frac{h(0)}{A(0)}-\frac{1}{A(0)}\right) \frac{1}{t^{3}}+O\left(\frac{1}{t^{4}}\right),
\end{aligned}
$$


where we have used the fact that the initial velocity is parallel to the critical position. We will next compute from (3.2.3) the Jacobian along a boundary trajectory near the critical point. For $i=1, \ldots, 3 N$, let $p_{i}(0)=x_{i}(0)$, the "position" components of $x(0)$. Working to first order, we start with

$$
x x^{\dagger}=\left(\begin{array}{cc}
x^{*} x^{* \dagger}+\frac{1}{t}\left(\frac{1}{\sqrt{A(0)}}\left[p(0) x^{* \dagger}+x^{*} p(0)^{\dagger}\right]-2 \frac{h(0)}{A(0)} x_{i}^{*} x^{* \dagger}\right) & \frac{1}{t} x^{*} x^{* \dagger} \\
\frac{1}{t} x^{*} x^{* \dagger} & O_{3 N}
\end{array}\right) .
$$

Multiplying on the right by $\left(\begin{array}{cc}O_{3 N} & I_{3 N} \\ I_{3 N} & O_{3 N}\end{array}\right)$ interchanges columns, and the Jacobian is

$$
\left(\begin{array}{cc}
-\frac{1}{t} I_{3 N} & I_{3 N}-x^{*} x^{* \dagger}-\frac{1}{t}\left(\frac{p(0) x^{* \dagger}+x^{*} p(0)^{\dagger}}{\sqrt{A(0)}}-\frac{2 h(0) x^{*} x^{* \dagger}}{A(0)}\right) \\
O_{3 N} & -\frac{1}{t}\left(I_{3 N}+x^{*} x^{* \dagger}\right)
\end{array}\right)
$$

to first order. Note that the upper right block doesn't contribute to the eigenvalues, since the lower right block vanishes.

The eigenvalues of the Jacobian are the zeroes of the characteristic polynomial, which is the determinant

$$
\left|\begin{array}{cc}
\left(\lambda+\frac{1}{t}\right) I_{3 N} & O_{3 N} \\
O_{3 N} & \lambda I_{3 N}+\frac{1}{t}\left(I_{3 N}+x^{*} x^{* \dagger}\right)
\end{array}\right|=\left(\lambda+\frac{1}{t}\right)^{3 N}\left|\lambda I_{3 N}+\frac{1}{t}\left(I_{3 N}+x^{*} x^{* \dagger}\right)\right|,
$$

all of whose zeroes are negative, since the zeroes of $\left|\lambda I_{3 N}+\frac{1}{t}\left(I_{3 N}+x^{*} x^{* \dagger}\right)\right|$ are negative real numbers: they are obviously the eigenvalues of the matrix $-\frac{1}{t}\left(I_{3 N}+x^{*} x^{* \dagger}\right)$, which are $-\frac{1}{t}$ times the eigenvalues of $I_{3 N}+x^{*} x^{* \dagger}$. But the equality $\left(I_{3 N}+x^{*} x^{* \dagger}\right)^{2}=I_{3 N}+3 x^{*} x^{* \dagger}=3\left(I_{3 N}+x^{*} x^{* \dagger}\right)-2 I_{3 N}$ implies that the eigenvalues of $I_{3 N}+x^{*} x^{* \dagger}$ are 1 and 2 . We conclude that the eigenvalues of the Jacobian along a boundary trajectory near a critical point are $-1 / t$ and $-2 / t$, which are negative along the trajectory and tend to 0 .

This gives a sense in which the trajectories in the boundary approaching the critical set attract nearby trajectories. Then as long as the segment of interest of a trajectory on the boundary does not intersect $\Delta$, and as long as it starts close enough to the boundary, it approaches the boundary. This means that all the trajectories in an open set containing the critical point set approach either the critical point set or $\Delta$.

\subsection{Approximate Solutions near the Boundary}

For a trajectory of (3.1.7) approaching a critical point in the boundary, there are two approximating systems to consider, both of which can be solved exactly. Those solutions to approximating systems will be shown to be approximate solutions of (3.1.7) in a sense to be made precise below. Consider a starting point $x(0)$ near the boundary. If we suppose it's close enough that we may assume $1-R^{2}=0$, the system (3.1.7) reduces to (3.2.1), whose solution in the interior takes exactly the same form as on the boundary (3.2.9). On the other hand, if we assume only that $1-R^{2}$ is small enough that its cube can be ignored, the system reduces to (3.2.2). As long as a solution to one of these approximate systems avoids collision, it can be expected to be a reasonable approximation to a trajectory of the full system (3.1.7). In fact, solutions to the second approximate system are asymptotic to solutions of (3.1.7). We uncompactify both of these approximate solutions; the first one turns out to be super-hyperbolic, and the second one will be shown to be asymptotic to the solutions we give in Chapter 5 to Celestial Mechanics equation. We compute series coefficients in $1 / t$ for both approximate interior trajectories that approach $\left(x^{* \dagger}, 0_{3 N}^{\dagger}\right)^{\dagger}$. We give necessary and sufficient conditions that the approximate trajectory avoids a collision and find the minimum particle separation. We show that the existence of a collision in the approximating trajectory is equivalent to the existence of an initial velocity difference anti-parallel to the corresponding initial position difference; for example, for some pair $a \neq b$

$$
x_{N+a}(0)-x_{N+b}(0)=-\sigma\left(x_{a}(0)-x_{b}(0)\right),
$$

for some $\sigma>0$. We show that without a collision, the minimum distance along the approximating trajectory has a positive infimum. 
We will form two new differential systems as approximations to (3.1.7); these systems are simple enough that closed-form solutions can be given. We will use the definition of Gingold and Tovbis [44] starting with the system

$$
\dot{z}=F(z, t), \text { or } \mathcal{N} z:=\dot{z}-F(z, t)=0,
$$

where $F \in C^{2}\left(\mathbb{B}_{\rho}\right) \times C\left[t_{0}, \infty\right), F$ and its derivatives are bounded on $\left[t_{0}, \infty\right)$, and $\mathbb{B}_{\rho}$ is the open ball of radius $\rho$ centered at the origin in $\mathbb{R}^{n}$. For our problem, $n$ is the dimension of the space of $x$, namely $n=6 N$, and the vector field $F$ is given by the right-hand side of (3.1.7).

Definition. For a given $m \in \mathbb{R}$ a function $z(t)$ is called an $m$-approximate solution of (3.3.1) if $z \in$ $C^{1}\left[t_{0}, \infty\right)$ and if there exists some $\delta>0$ such that

$$
\mathcal{N} z=O\left(t^{-m-\delta}\right), \text { as } t \rightarrow \infty .
$$

We will find solutions to our approximating systems and show that these solutions are $m$-approximate solutions to (3.1.7).

3.3.1. An approximating system. We begin by extending the boundary system (3.2.1) to the interior.

LEMma 24. The approximating system

$$
\begin{aligned}
\dot{x}_{i} & =-h x_{i}+x_{N+i} \\
\dot{x}_{N+i} & =-h x_{N+i}
\end{aligned}
$$

for $1-R^{2}<<1$ has an analytic solution

$$
x_{i}=\frac{x_{i}(0)+x_{N+i}(0) t}{\sqrt{1+2 h(0) t+A(0) t^{2}}}, \quad x_{N+i}=\frac{x_{N+i}(0)}{\sqrt{1+2 h(0) t+A(0) t^{2}}},
$$

whose power series in $1 / t$ is convergent for $t \in(T, \infty)$, for some finite $T$.

Proof. The following calculation is similar to that on the boundary in the previous Section and produces the same solution (3.2.9) to (3.2.1). It is easy to see that

$$
x=E\left(\begin{array}{cc}
I_{3 N} & t I_{3 N} \\
O_{3 N} & I_{3 N}
\end{array}\right) x(0)
$$

is a solution, with $E=\exp \int_{0}^{t} h d s$. Now a straightforward calculation from (3.3.2) gives

$$
\frac{1}{2} \frac{d R^{2}}{d t}=x^{\dagger} \dot{x}=-R^{2} h+h=\left(1-R^{2}\right) h,
$$

which leads to

$$
\begin{aligned}
\frac{d}{d t} \log \left(1-R^{2}\right) & =-\frac{\frac{d R^{2}}{d t}}{1-R^{2}}=-2 h \\
1-R^{2} & \left.=\left(1-R(0)^{2}\right)\right) \exp \left(-2 \int_{0}^{t} h d s\right)=E^{2}\left(1-R(0)^{2}\right) .
\end{aligned}
$$

Taking squares of magnitudes of (3.3.4) gives

$$
\begin{gathered}
R^{2}=\sum_{i=1}^{N}\left(x_{i}^{\dagger} x_{i}+x_{N+i}^{\dagger} x_{N+i}\right) \\
=E^{2} \sum_{i=1}^{N}\left(x_{i}^{\dagger}(0) x_{i}(0)+2 t x_{i}^{\dagger}(0) x_{N+i}(0)+t^{2} x_{N+i}^{\dagger}(0) x_{N+i}(0)+x_{N+i}^{\dagger}(0) x_{N+i}(0)\right) \\
=E^{2}\left(R(0)^{2}+2 h(0) t+A(0) t^{2}\right),
\end{gathered}
$$




$$
E^{2}=\frac{1-R^{2}}{1-R(0)^{2}}=\frac{R^{2}}{R(0)^{2}+2 h(0) t+A(0) t^{2}}
$$

This is an equation we can solve for $R^{2}$ after cross-multiplying,

so that

$$
\begin{gathered}
\left(1-R^{2}\right)\left(R(0)^{2}+2 h(0) t+A(0) t^{2}\right)=R^{2}\left(1-R(0)^{2}\right) \\
R^{2}=\frac{R(0)^{2}+2 h(0) t+A(0) t^{2}}{1+2 h(0) t+A(0) t^{2}}
\end{gathered}
$$

$$
E^{2}=\frac{1-R^{2}}{1-R(0)^{2}}=\frac{1+2 h(0) t+A(0) t^{2}-\left(R(0)^{2}+2 h(0) t+A(0) t^{2}\right)}{\left(1-R(0)^{2}\right)\left(1+2 h(0) t+A(0) t^{2}\right)}=\frac{1}{1+2 h(0) t+A(0) t^{2}},
$$

confirming (3.3.3). $E$ is clearly analytic for sufficiently large $t$, so $x$ is, too.

This solution has $6 N$ parameters $x(0)$ constrained only by the ten integrals of the motion. Note that we still have $x \rightarrow\left(x_{N+i}(0)^{\dagger} / \sqrt{A(0)}, 0_{3 N}^{\dagger}\right)^{\dagger}$ as $t \rightarrow \infty$, which is a critical point $\left(x^{* \dagger}, 0_{3 N}^{\dagger}\right)^{\dagger}$ on the boundary; that is, this trajectory approaches the boundary as $t \rightarrow \infty$. We note that this system also has critical points in the interior of the ball. The critical point set is $\left\{x \mid x_{N+i}=0_{3}\right\}$; however, as we have seen all trajectories of this system tend to fixed points on the boundary. We can give closed-form expressions for the quantities $h$ and $A$ from the formal solution (3.3.4)

$$
h=E^{2} \sum_{i=1}^{N}\left(x_{i}(0)+t x_{N+i}(0)\right)^{\dagger} x_{N+i}(0)=\frac{h(0)+A(0) t}{1+2 h(0) t+A(0) t^{2}}, \quad A=\frac{A(0)}{1+2 h(0) t+A(0) t^{2}},
$$

and $R^{2}$ from (3.3.6)

$$
1-R^{2}=\frac{1-R(0)^{2}}{1+2 h(0) t+A(0) t^{2}}
$$

Let us refer to this solution (3.3.3) to the approximate system (3.3.2) as $\tilde{x}$.

Per the definition given at the beginning of this Section, we can now see that $\tilde{x}$ is a $m$-approximate solution for $m<3$. First, on adding and subtracting $h$, we have

SO

$$
\theta=-h+h-\frac{2 h}{1+R^{2}}-\frac{2\left(1-R^{2}\right)^{3} L}{1+R^{2}}=-h-\frac{1-R^{2}}{1+R^{2}} h-\frac{2\left(1-R^{2}\right)^{3} L}{1+R^{2}}
$$

$$
\mathcal{N} \tilde{x}=\dot{\tilde{x}}-\left(\begin{array}{cc}
\theta I_{3 N} & I_{3 N} \\
O_{3 N} & \theta I_{3 N}
\end{array}\right) \tilde{x}-\left(\begin{array}{c}
O_{3 N} \\
\left(1-R^{2}\right)^{3} G
\end{array}\right)=\left(\frac{1-R^{2}}{1+R^{2}} h+\frac{2\left(1-R^{2}\right)^{3} L}{1+R^{2}}\right) \tilde{x}-\left(\begin{array}{c}
O_{3 N} \\
\left(1-R^{2}\right)^{3} G
\end{array}\right),
$$

where all the quantities $(h, R, G, L)$ are evaluated along $\tilde{x}$. Since $\left(1-R^{2}\right) h=O\left(1 / t^{3}\right)$, we have

$$
\mathcal{N} \tilde{x}=O\left(\frac{1}{t^{3}}\right)
$$

confirming that $\tilde{x}$ is an $m$-approximate solution for $m<3$.

We will now un-compactify $\tilde{x}$.

$$
\tilde{y}=\frac{1}{1-R^{2}} \tilde{x}=\frac{E}{1-R^{2}}\left(\begin{array}{cc}
I_{3 N} & t I_{3 N} \\
O_{3 N} & I_{3 N}
\end{array}\right) x(0) .
$$

From (3.3.6) we have

so

$$
\frac{E}{1-R^{2}}=\frac{1}{\sqrt{1-R^{2}}} \frac{1}{\sqrt{1-R(0)^{2}}}=\frac{\sqrt{1-R(0)^{2}}}{\sqrt{1-R^{2}}} \frac{1}{1-R(0)^{2}}=\frac{1}{E} \frac{1}{1-R(0)^{2}},
$$

$$
\tilde{y}=\frac{1}{E}\left(\begin{array}{cc}
I_{3 N} & t I_{3 N} \\
O_{3 N} & I_{3 N}
\end{array}\right) y(0)
$$

Note

$$
\frac{1}{E}=\sqrt{1+2 h_{x}(0) t+A_{x}(0) t^{2}}
$$


where the subscript $x$ serves to remind us that these quantities derive from the trajectory $\tilde{x}$. We want to express this in terms of $y(0)$. We have from (3.1.3) that

$$
1-R^{2}=\frac{2}{1+\sqrt{1+4 r^{2}}}
$$

so that

$$
\left(1-R^{2}\right)^{2}=\frac{2}{1+\sqrt{1+4 r^{2}}+2 r^{2}} .
$$

Then we have

$$
h_{x}=\frac{2}{1+\sqrt{1+4 r^{2}}+2 r^{2}} h_{y} \text { and } A_{x}=\frac{2}{1+\sqrt{1+4 r^{2}}+2 r^{2}} A_{y}
$$

where the corresponding quantities for $\tilde{y} \in \mathbb{R}^{6 N}$ are defined

$$
h_{y}=\sum_{i=1}^{N} y_{i}^{\dagger} y_{N+i} \quad A_{y}=\sum_{i=1}^{N} y_{N+i}^{\dagger} y_{N+i} .
$$

Returning to the computation of $\tilde{y}$

$$
\tilde{y}=\left\{1+\frac{4 h_{y}(0) t+2 A_{y}(0) t^{2}}{1+\sqrt{1+4 r(0)^{2}}+2 r(0)^{2}}\right\}^{1 / 2}\left(\begin{array}{cc}
I_{3 N} & t I_{3 N} \\
O_{3 N} & I_{3 N}
\end{array}\right) y(0),
$$

which is super-hyperbolic [99], since the particle distances from the origin grow like $t^{2}$. The trajectory (3.3.8) must satisfy the uncompactification of (3.3.2)

$$
\begin{gathered}
\dot{y}=\frac{\dot{x}}{1-R^{2}}-\left[\frac{d}{d t}\left(1-R^{2}\right)\right] \frac{x}{\left(1-R^{2}\right)^{2}}=\left(\begin{array}{cc}
-h_{x} I_{3 N} & I_{3 N} \\
O_{3 N} & -h_{x} I_{3 N}
\end{array}\right) \frac{x}{1-R^{2}}+2 h_{x} y \\
\dot{y}=\left(\begin{array}{cc}
O_{3 N} & I_{3 N} \\
O_{3 N} & O_{3 N}
\end{array}\right) y+\frac{2}{1+\sqrt{1+4 r^{2}}+2 r^{2}} h_{y} y
\end{gathered}
$$

which is consistent with (3.3.8), as can be seen on differentiating (3.3.7)

$$
\begin{gathered}
\dot{\tilde{y}}=\frac{d}{d t}\left(\frac{1}{E}\right)\left(\begin{array}{cc}
I_{3 N} & t I_{3 N} \\
O_{3 N} & I_{3 N}
\end{array}\right) y(0)+\frac{1}{E}\left(\begin{array}{cc}
O_{3 N} & I_{3 N} \\
O_{3 N} & O_{3 N}
\end{array}\right) y(0) \\
\frac{d}{d t}\left(\frac{1}{E}\right)=\frac{1}{2} \frac{2 h_{x}(0)+2 A_{x}(0) t}{\sqrt{1+2 h_{x}(0) t+A_{x}(0) t^{2}}}=E\left(h_{x}(0)+A_{x}(0) t\right)=\frac{h_{x}}{E},
\end{gathered}
$$

so

$$
\dot{\tilde{y}}=\frac{h_{x}}{E}\left(\begin{array}{cc}
I_{3 N} & t I_{3 N} \\
O_{3 N} & I_{3 N}
\end{array}\right) y(0)+\left(\begin{array}{cc}
O_{3 N} & I_{3 N} \\
O_{3 N} & O_{3 N}
\end{array}\right) y=h_{x} y+\left(\begin{array}{cc}
O_{3 N} & I_{3 N} \\
O_{3 N} & O_{3 N}
\end{array}\right) y,
$$

which matches (3.3.9).

The asymptotic behavior of this trajectory as $t \rightarrow \infty$ can be analyzed just as the trajectory in the boundary in the previous Section, so long as there is no collision. The denominator of $E$ may be expressed as

$$
\sqrt{A(0)} t \sqrt{1+\frac{2 h(0) t+1}{A(0) t^{2}}}
$$

so expanding $E$ in powers of $1 / t$

$$
E=\frac{1}{\sqrt{A(0)} t}\left(1+\frac{2 h(0) t+1}{A(0) t^{2}}\right)^{-1 / 2}=\frac{1}{\sqrt{A(0)}}\left(\frac{1}{t}-\frac{h(0)}{A(0)} \frac{1}{t^{2}}+\left(\frac{3}{2} \frac{h(0)^{2}}{A(0)^{2}}-\frac{1}{2 A(0)}\right) \frac{1}{t^{3}}\right)+O\left(\frac{1}{t^{4}}\right)
$$

and the asymptotic behavior has the same lowest terms as (3.2.10): 


$$
\begin{aligned}
x_{i}(t) & =x_{i}^{*}+\left(\frac{x_{i}(0)}{\sqrt{A(0)}}-\frac{h(0)}{A(0)} x_{i}^{*}\right) \frac{1}{t}+\left(\frac{x_{i}(0)}{\sqrt{A(0)}}+\left(\frac{3}{2} \frac{h(0)^{2}}{A(0)^{2}}-\frac{1}{2 A(0)}\right) x_{i}^{*}\right) \frac{1}{t^{2}}+O\left(\frac{1}{t^{3}}\right) \\
x_{N+i}(t) & =\left(\frac{1}{t}-\frac{h(0)}{A(0) t^{2}}+\left(\frac{3}{2} \frac{h(0)^{2}}{A(0)^{2}}-\frac{1}{2 A(0)}\right) \frac{1}{t^{3}}\right) x_{i}^{*}+O\left(\frac{1}{t^{4}}\right) \\
10) \quad h & =\frac{1}{t}-\frac{h(0)}{A(0) t^{2}}+\left(3 \frac{h(0)^{2}}{A(0)^{2}}+\frac{h(0)}{A(0)}-\frac{1}{A(0)}\right) \frac{1}{t^{3}}+O\left(\frac{1}{t^{4}}\right),
\end{aligned}
$$

We will need one additional asymptotic formula:

$$
\begin{gathered}
\frac{1-R^{2}}{1-R(0)^{2}}=\frac{1}{A(0) t^{2}}\left(1+\frac{2 h(0)}{A(0) t}+\frac{1}{A(0) t^{2}}\right)^{-1}=\frac{1}{A(0) t^{2}}\left(1-\frac{2 h(0)}{A(0) t}\right)+O\left(\frac{1}{t^{4}}\right) \\
=\frac{1}{A(0) t^{2}}-\frac{2 h(0)}{A(0)^{2} t^{3}}+O\left(\frac{1}{t^{4}}\right) .
\end{gathered}
$$

3.3.2. A closer approximation. Next we will approximate (3.1.7) more closely, neglecting terms of order $O\left(\left(1-R^{2}\right)^{3}\right)$, and we exhibit our second, closer approximate system, which also has a closed-form solution analytic in $1 / t$. We will see that solutions to this approximating system, to be called $\bar{x}$ in the sequel, approach the boundary as $t \rightarrow \infty$, and we will in the next Chapter seek solutions to the full compactified system (3.1.7) as perturbations of this one.

One justification for describing (3.3.11) as an approximating system to the full compactified system (3.1.7) is that the two systems have the same Jacobian at critical points on the boundary.

LEMMA 25. The approximating system

$$
\begin{aligned}
\dot{x}_{i} & =-\frac{2}{1+R^{2}} h x_{i}+x_{N+i} \\
\dot{x}_{N+i} & =-\frac{2}{1+R^{2}} h x_{N+i} .
\end{aligned}
$$

for $1-R^{2} \ll 1$ has an analytic solution given in closed form

$$
\begin{aligned}
\bar{x}_{i} & =2 \frac{x_{i}(0)+x_{N+i}(0) t}{1-R(0)^{2}+\sqrt{\left(1+R(0)^{2}\right)^{2}+8 h(0) t+4 A(0) t^{2}}} \\
\bar{x}_{N+i} & =2 \frac{x_{N+i}(0)}{1-R(0)^{2}+\sqrt{\left(1+R(0)^{2}\right)^{2}+8 h(0) t+4 A(0) t^{2}}},
\end{aligned}
$$

whose power series in $1 / t$ is convergent for $t \in(T, \infty)$, for some finite $T$.

Proof. We can solve (3.3.11) the same way: let

$$
x=E\left(\begin{array}{cc}
I_{3 N} & t I_{3 N} \\
O_{3 N} & I_{3 N}
\end{array}\right) x(0)
$$

and differentiate both sides

$$
\dot{x}=\dot{E}\left(\begin{array}{cc}
I_{3 N} & t I_{3 N} \\
O_{3 N} & I_{3 N}
\end{array}\right) x(0)+E\left(\begin{array}{cc}
O_{3 N} & I_{3 N} \\
O_{3 N} & O_{3 N}
\end{array}\right) x(0)=\frac{\dot{E}}{E} x+\left(\begin{array}{cc}
O_{3 N} & I_{3 N} \\
O_{3 N} & O_{3 N}
\end{array}\right) x .
$$

That has to match (3.3.11) so we have

$$
\frac{\dot{E}}{E}=-\frac{2 h}{1+R^{2}}, \text { so } E=\exp \left(-2 \int_{0}^{t} \frac{h}{1+R^{2}} d s\right) .
$$

Taking squares of magnitudes now gives

$$
R^{2}=E^{2} \sum_{i=1}^{N}\left(x_{i}^{\dagger}(0) x_{i}(0)+2 t x_{i}^{\dagger}(0) x_{N+i}(0)+t^{2} x_{N+i}^{\dagger}(0) x_{N+i}(0)+x_{N+i}^{\dagger}(0) x_{N+i}(0)\right)
$$




$$
=E^{2}\left(R(0)^{2}+2 h(0) t+A(0) t^{2}\right)
$$

or

$$
E^{2}=\frac{R^{2}}{R(0)^{2}+2 h(0) t+A(0) t^{2}} .
$$

A straightforward calculation from (3.3.11)

$$
\frac{1}{2} \frac{d R^{2}}{d t}=x^{\dagger} \dot{x}=-\frac{2 h R^{2}}{1+R^{2}}+h=\frac{1-R^{2}}{1+R^{2}} h
$$

leads to

$$
\frac{d}{d t} \log \left(1-R^{2}\right)=-\frac{\frac{d R^{2}}{d t}}{1-R^{2}}=-2 \frac{h}{1+R^{2}},
$$

with solution

$$
\frac{1-R^{2}}{1-R(0)^{2}}=\exp \left(-2 \int_{0}^{t} \frac{h}{1+R^{2}} d t\right)=E .
$$

Starting with (3.3.13), we add and subtract 1 in the numerator and then multiply the top and bottom by $1-R(0)^{2}$

$$
E^{2}=\frac{R^{2}-1}{R(0)^{2}+2 h(0) t+A(0) t^{2}} \frac{1-R(0)^{2}}{1-R(0)^{2}}+\frac{1}{R(0)^{2}+2 h(0) t+A(0) t^{2}},
$$

which we can express as a quadratic in $E$

$$
E^{2}+\frac{1-R(0)^{2}}{R(0)^{2}+2 h(0) t+A(0) t^{2}} E-\frac{1}{R(0)^{2}+2 h(0) t+A(0) t^{2}}=0 .
$$

For the moment, let $d=R(0)^{2}+2 h(0) t+A(0) t^{2}$, then the quadratic formula provides

Or

$$
E=\frac{-\frac{1-R(0)^{2}}{d}+\sqrt{\frac{\left(1-R(0)^{2}\right)^{2}}{d^{2}}+\frac{4}{d}}}{2}=\frac{-\left(1-R(0)^{2}\right)+\sqrt{\left(1-R(0)^{2}\right)^{2}+4 d}}{2 d},
$$

$$
E=\frac{-\left(1-R(0)^{2}\right)+\sqrt{\left(1+R(0)^{2}\right)^{2}+8 h(0) t+4 A(0) t^{2}}}{2 R(0)^{2}+4 h(0) t+2 A(0) t^{2}} .
$$

We multiply the numerator and denominator by the conjugate of the numerator to obtain the preferred form

$$
E=\frac{1-R^{2}}{1-R(0)^{2}}=\frac{2}{1-R(0)^{2}+\sqrt{\left(1+R(0)^{2}\right)^{2}+8 h(0) t+4 A(0) t^{2}}} .
$$

This gives the closed form expressions promised. Obviously, for $T$ sufficiently large, the solution is analytic in $1 / t$ on $(T, \infty)$, since $|h| \leq 1 / 2$ and $0<A(0)<1$.

This solution has $6 N$ parameters $x(0)$ constrained only by the ten integrals of the motion. Note that we still have $x \rightarrow\left(x_{N+i}(0)^{\dagger} / \sqrt{A(0)}, 0_{3 N}^{\dagger}\right)^{\dagger}$ as $t \rightarrow \infty$, which is a critical point $\left(x^{* \dagger}, 0_{3 N}^{\dagger}\right)^{\dagger}$ on the boundary; that is, this trajectory approaches the boundary as $t \rightarrow \infty$. We note that this system also has critical points in the interior of the ball. The critical point set is $\left\{x \mid x_{N+i}=0_{3}\right\}$; however, as we have seen all trajectories of this system tend to fixed points on the boundary. We can express the evolution of $h$ and $A$, as well:

$$
\begin{aligned}
h & =E^{2}(h(0)+t A(0)) \\
A & =E^{2} A(0) .
\end{aligned}
$$

We can compute $E^{2}$

$$
\begin{gathered}
\frac{4}{E^{2}}=\left(1-R(0)^{2}\right)^{2}+2\left(1-R(0)^{2}\right) \sqrt{\left(1+R(0)^{2}\right)^{2}+8 h(0) t+4 A(0) t^{2}}+\left(1+R(0)^{2}\right)^{2}+8 h(0) t+4 A(0) t^{2} \\
=2+2 R(0)^{4}+2\left(1-R(0)^{2}\right) \sqrt{\left(1+R(0)^{2}\right)^{2}+8 h(0) t+4 A(0) t^{2}}+8 h(0) t+4 A(0) t^{2} \\
E^{2}=\frac{2}{1+R(0)^{4}+\left(1-R(0)^{2}\right) \sqrt{\left(1+R(0)^{2}\right)^{2}+8 h(0) t+4 A(0) t^{2}}+4 h(0) t+2 A(0) t^{2}} .
\end{gathered}
$$


We can give closed-form expressions for the quantities $h$ and $A$ from the formal solution (3.3.4)

$$
\begin{aligned}
& h=\frac{2 h(0)+2 A(0) t}{1+R(0)^{4}+\left(1-R(0)^{2}\right) \sqrt{\left(1+R(0)^{2}\right)^{2}+8 h(0) t+4 A(0) t^{2}}+4 h(0) t+2 A(0) t^{2}} \\
& A=\frac{2 A(0)}{1+R(0)^{4}+\left(1-R(0)^{2}\right) \sqrt{\left(1+R(0)^{2}\right)^{2}+8 h(0) t+4 A(0) t^{2}}+4 h(0) t+2 A(0) t^{2}} .
\end{aligned}
$$

Per the definition given at the beginning of this Section, we can now see that $\bar{x}$ is a $m$-approximate solution for $m<3$.

$$
\mathcal{N} \bar{x}=\dot{\bar{x}}-\left(\begin{array}{cc}
\theta I_{3 N} & I_{3 N} \\
O_{3 N} & \theta I_{3 N}
\end{array}\right) \bar{x}-\left(\begin{array}{c}
O_{3 N} \\
\left(1-R^{2}\right)^{3} G
\end{array}\right)=\frac{2\left(1-R^{2}\right)^{3} L}{1+R^{2}} \bar{x}-\left(\begin{array}{c}
O_{3 N} \\
\left(1-R^{2}\right)^{3} G
\end{array}\right)
$$

where again all the quantities $(h, R, G, L)$ are evaluated along $\bar{x}$. Since $\left(1-R^{2}\right)^{3} G=O\left(1 / t^{3}\right)$, we have

$$
\mathcal{N} \bar{x}=O\left(\frac{1}{t^{3}}\right)
$$

confirming that $\bar{x}$ is also an $m$-approximate solution for $m<3$.

We will now un-compactify $\bar{x}$.

$$
\bar{y}=\frac{\bar{x}}{1-R^{2}}=\frac{1}{1-R^{2}} E\left(\begin{array}{cc}
I_{3 N} & t I_{3 N} \\
O_{3 N} & I_{3 N}
\end{array}\right) x(0)=\left(\begin{array}{cc}
I_{3 N} & t I_{3 N} \\
O_{3 N} & I_{3 N}
\end{array}\right) \frac{1}{1-R^{2}} x(0)=\left(\begin{array}{cc}
I_{3 N} & t I_{3 N} \\
O_{3 N} & I_{3 N}
\end{array}\right) y(0),
$$

or

$$
\begin{aligned}
\bar{y}_{i} & =y_{i}(0)+t y_{N+i}(0) \\
\bar{y}_{N+i} & =y_{N+i}(0) .
\end{aligned}
$$

This trajectory is hyperbolic, since the particle distances from the origin grow like $t$. The expanding solutions that we construct in Chapter 5 will be seen to be asymptotic to $\bar{y}$. Of course, the trajectory (3.3.17) must satisfy the uncompactification of (3.3.2)

$$
\dot{y}=\frac{\dot{x}}{1-R^{2}}-\left[\frac{d}{d t}\left(1-R^{2}\right)\right] \frac{x}{\left(1-R^{2}\right)^{2}}=\left[\frac{-2 h_{x}}{1+R^{2}}+\left(\begin{array}{cc}
O_{3 N} & I_{3 N} \\
O_{3 N} & O_{3 N}
\end{array}\right)\right] \frac{x}{1-R^{2}}+2 \frac{1-R^{2}}{1+R^{2}} h_{x} \frac{x}{\left(1-R^{2}\right)^{2}},
$$

or

$$
\dot{y}=\left(\begin{array}{cc}
O_{3 N} & I_{3 N} \\
O_{3 N} & O_{3 N}
\end{array}\right) y
$$

which is consistent with (3.3.17).

For future reference, we evaluate the asymptotic behavior of this trajectory as $t \rightarrow \infty$, again assuming there is no collision. As long as $t$ is large enough, the radical in (3.3.12) is well defined. Factoring out the leading term, the denominator of $E$ may be expressed as

$$
2 \sqrt{A(0)} t\left(\sqrt{1+\frac{2 h(0)}{A(0) t}+\frac{\left(1+R(0)^{2}\right)^{2}}{4 A(0) t^{2}}}+\frac{1-R(0)^{2}}{2 \sqrt{A(0)} t}\right) .
$$

The big square root has a power series, starting with

$$
1+\frac{1}{t} \frac{h(0)}{A(0)}+\frac{1}{t^{2}}\left[\frac{\left(1+R(0)^{2}\right)^{2}}{8 A(0)}-\frac{h(0)^{2}}{2 A(0)^{2}}\right]+O\left(\frac{1}{t^{3}}\right)
$$

convergent for

$$
\left|\frac{2 h(0)}{A(0) t}+\frac{\left(1+R(0)^{2}\right)^{2}}{4 A(0) t^{2}}\right|<1
$$

which obviously holds for sufficiently large $t$.

Including the $1-R(0)^{2}$ term, the denominator is

$$
\frac{2}{E}=2 \sqrt{A(0)} t\left(1+\frac{1}{t}\left[\frac{h(0)}{A(0)}+\frac{1-R(0)^{2}}{2 \sqrt{A(0)}}\right]+\frac{1}{t^{2}}\left[\frac{\left(1+R(0)^{2}\right)^{2}}{8 A(0)}-\frac{h(0)^{2}}{2 A(0)^{2}}\right]+O\left(\frac{1}{t^{3}}\right)\right),
$$


so we have the first few terms of the series for $E$, convergent for sufficiently large $t$ :

$$
\begin{gathered}
E=\frac{1}{\sqrt{A(0) t}}\left\{1-\frac{1}{t}\left[\frac{h(0)}{A(0)}+\frac{1-R(0)^{2}}{2 \sqrt{A(0)}}\right]\right. \\
\left.+\frac{1}{t^{2}}\left[\frac{3 h(0)^{2}}{2 A(0)^{2}}+\frac{h(0)\left(1-R(0)^{2}\right)}{A(0)^{3 / 2}}-\frac{1-6 R(0)^{2}+R(0)^{4}}{8 A(0)}\right]\right\}+O\left(\frac{1}{t^{4}}\right) .
\end{gathered}
$$

We will also need the first few terms of $E^{2}$

$$
E^{2}=\frac{1}{A(0) t^{2}}\left(1-\frac{2 h(0)}{A(0) t}-\frac{1-R(0)^{2}}{\sqrt{A(0)} t}\right)+O\left(\frac{1}{t^{4}}\right)=\frac{1}{A(0) t^{2}}-\left(\frac{2 h(0)}{A(0)^{2}}+\frac{1-R(0)^{2}}{A(0)^{3 / 2}}\right) \frac{1}{t^{3}}+O\left(\frac{1}{t^{4}}\right)
$$

It is easy to see that for this case also, we have that

$$
h=\frac{1}{t}-\left(\frac{h(0)}{A(0)}+\frac{1-R(0)^{2}}{\sqrt{A(0)}}\right) \frac{1}{t^{2}}+O\left(\frac{1}{t^{3}}\right) \quad A=\frac{1}{t^{2}}-\left(\frac{2 h(0)}{A(0)}+\frac{1-R(0)^{2}}{\sqrt{A(0)}}\right) \frac{1}{t^{3}}+O\left(\frac{1}{t^{4}}\right)
$$

as $t \rightarrow \infty$.

We can express the first few terms of $x$ using (3.3.12) as

$$
\begin{aligned}
x_{i}=x_{i}^{*}+ & \frac{1}{t}\left(\frac{x_{i}(0)}{\sqrt{A(0)}}-\left[\frac{h(0)}{A(0)}+\frac{1-R(0)^{2}}{2 \sqrt{A(0)}}\right] x_{i}^{*}\right)-\frac{1}{t^{2}}\left[\frac{h(0)}{A(0)}+\frac{1-R(0)^{2}}{2 \sqrt{A(0)}}\right] \frac{x_{i}(0)}{\sqrt{A(0)}} \\
& +\frac{1}{t^{2}}\left[\frac{3 h(0)^{2}}{2 A(0)^{2}}+\frac{h(0)\left(1-R(0)^{2}\right)}{A(0)^{3 / 2}}-\frac{1-6 R(0)^{2}+R(0)^{4}}{8 A(0)}\right] x^{*}+O\left(\frac{1}{t^{3}}\right) \\
x_{N+i}=\frac{1}{t} x_{i}^{*} & -\frac{1}{t^{2}}\left[\frac{h(0)}{A(0)}+\frac{1-R(0)^{2}}{2 \sqrt{A(0)}}\right] x_{i}^{*} \\
+ & \frac{1}{t^{3}}\left[\frac{3 h(0)^{2}}{2 A(0)^{2}}+\frac{h(0)\left(1-R(0)^{2}\right)}{A(0)^{3 / 2}}-\frac{1-6 R(0)^{2}+R(0)^{4}}{8 A(0)}\right] x_{i}^{*}+O\left(\frac{1}{t^{4}}\right) .
\end{aligned}
$$

Moreover,

$$
\frac{1-R^{2}}{1-R(0)^{2}}=\frac{1}{\sqrt{A(0)} t}-\frac{1}{t^{2}}\left(\frac{h(0)}{A(0)^{3 / 2}}+\frac{1-R(0)^{2}}{2 A(0)}\right)+O\left(\frac{1}{t^{3}}\right)
$$

which means that this trajectory approaches the boundary more slowly than $\tilde{x}$ does.

Given that $x$ has a series expansion convergent for sufficiently large $t$,

$$
x=\left(\begin{array}{c}
x^{*}+a_{1} / t+a_{2} / t^{2}+\ldots \\
x^{*}\left[B_{1} / t+B_{2} / t^{2}+B_{3} / t^{3}+\ldots\right]
\end{array}\right),
$$

where the $a_{k}$ are $3 N$-vectors, let $A_{k}=a_{k}^{\dagger} x^{*}$. We have computed the first few terms just above, which we record for later use: 


$$
\begin{aligned}
(3.3 .19) a_{1 i}= & \frac{x_{i}(0)}{\sqrt{A(0)}}-\left(\frac{h(0)}{A(0)}+\frac{1-R(0)^{2}}{2 \sqrt{A(0)}}\right) x_{i}^{*}, \quad A_{1}=-\frac{1-R(0)^{2}}{2 \sqrt{A(0)}}<0 \\
(3.3 .20) a_{2 i}= & -\left[\frac{h(0)}{A(0)}+\frac{1-R(0)^{2}}{2 \sqrt{A(0)}}\right] \frac{x_{i}(0)}{\sqrt{A(0)}}+\left[\frac{3 h(0)^{2}}{2 A(0)^{2}}+\frac{h(0)\left(1-R(0)^{2}\right)}{A(0)^{3 / 2}}-\frac{1-6 R(0)^{2}+R(0)^{4}}{8 A(0)}\right] x_{i}^{*} \\
A_{2}= & \frac{h(0)^{2}}{2 A(0)^{2}}+\frac{h(0)\left(1-R(0)^{2}\right)}{2 A(0)^{3 / 2}}-\frac{1-6 R(0)^{2}+R(0)^{4}}{8 A(0)} \\
B_{1}= & 1 \\
(3.3 .21) B_{2}= & -\frac{h(0)}{A(0)}-\frac{1-R(0)^{2}}{2 \sqrt{A(0)}} ; \quad A_{1}-B_{2}=\frac{h(0)}{A(0)} \\
B_{3}= & \frac{3 h(0)^{2}}{2 A(0)^{2}}+\frac{h(0)\left(1-R(0)^{2}\right)}{A(0)^{3 / 2}}-\frac{1-6 R(0)^{2}+R(0)^{4}}{8 A(0)} . \\
& B_{3}-A_{2}=\frac{h(0)^{2}}{A(0)^{2}}+\frac{h(0)\left(1-R(0)^{2}\right)}{2 A(0)^{3 / 2}}=-\frac{h(0)}{A(0)} B_{2}=\left(B_{2}-A_{1}\right) B_{2}
\end{aligned}
$$

Note that $1-6 R(0)^{2}+R(0)^{4}<0$ provided $R(0)^{2}>\sqrt{2}-1 \sim 0.41$. So $A_{2}$ and $B_{3}$ are both positive.

Note that since each $\bar{x}_{i}$ stays in the linear span of $x_{i}(0)$ and $x_{i}^{*}$, and each $\bar{x}_{N+1}$ is always parallel to $x_{i}^{*}$, the motion of $\bar{x}$ in the ball $\mathbb{B}^{6 N}$ is actually confined to a three-dimensional subspace, the linear span of the three independent vectors

$$
\left(\begin{array}{c}
x^{*} \\
0_{3 N}
\end{array}\right),\left(\begin{array}{c}
v_{2} \\
0_{3 N}
\end{array}\right),\left(\begin{array}{c}
0_{3 N} \\
x^{*}
\end{array}\right)
$$

where $v_{2}$ is the vector formed via the Graham-Schmidt process starting with $v_{1}=x^{*}$ and $\bar{x}_{j}\left(t_{0}\right)$. We expect that asymptotically something similar is true for solutions $x$ of the compactified system (3.1.7) that are asymptotic to $\bar{x}$. To be explicit

$$
\begin{aligned}
v_{2 j} & =\frac{\sqrt{A\left(t_{0}\right)} x_{j}\left(t_{0}\right)-h\left(t_{0}\right) x_{j}^{*}}{\sqrt{A\left(t_{0}\right)\left(R\left(t_{0}\right)^{2}-A\left(t_{0}\right)\right)-h\left(t_{0}\right)^{2}}}, \text { for } 1 \leq j \leq 3 N \\
x_{j}\left(t_{0}\right) & =\frac{h\left(t_{0}\right)}{\sqrt{A\left(t_{0}\right)}} x_{j}^{*}+\sqrt{R\left(t_{0}\right)^{2}-A\left(t_{0}\right)-\frac{h\left(t_{0}\right)^{2}}{A\left(t_{0}\right)}} v_{2 j} .
\end{aligned}
$$

Next we analyze the initial conditions for this approximation that lead to collision in finite time and those that don't.

Definition. Two vectors $v_{1}$ and $v_{2}$ are anti-parallel if there is a positive number $\beta$ such that $v_{1}=-\beta v_{2}$.

If $x_{a}(\sigma)=x_{b}(\sigma)$, for some $0<\sigma<\infty$ and some $a \neq b$, then from (3.3.12), we must have

$$
x_{a}(0)+\sigma x_{N+a}(0)=x_{b}(0)+\sigma x_{N+b}(0) .
$$

Then

$$
\sigma\left(x_{N+a}(0)-x_{N+b}(0)\right)=x_{b}(0)-x_{a}(0),
$$

so the $a b$ initial velocity difference is anti-parallel to the initial position difference. The reasoning works in reverse as well, so for both $\tilde{x}$ and $\bar{x}$, a collision between particles $a$ and $b$ in the future is equivalent to the $a b$ initial velocity difference being anti-parallel to the initial position difference.

Now we consider trajectories that approach a collision as $t \rightarrow \infty$. This means that for some $a \neq b$,

$$
x_{a}^{*}=\lim x_{a}=\lim x_{b}=x_{b}^{*},
$$

and a collision as $t \rightarrow \infty$ is equivalent to $x^{*}$ being a point of collision, or equivalently that a pair of initial velocities are equal. 
Suppose that $\bar{x}$ does not experience a collision nor approach one, and consider the particle separation

$$
\begin{aligned}
\left\|x_{a}-x_{b}\right\|^{2}= & E^{2}\left\|x_{a}(0)-x_{b}(0)+t\left(x_{N+a}(0)-x_{N+b}(0)\right)\right\|^{2} . \\
= & E^{2}\left\{\left\|x_{a}(0)-x_{b}(0)\right\|^{2}+2 t\left(x_{a}(0)-x_{b}(0)\right)^{\dagger}\left(x_{N+a}(0)-x_{N+b}(0)\right)\right. \\
\quad & \left.\quad+t^{2}\left\|x_{N+a}(0)-x_{N+b}(0)\right\|^{2}\right\} \\
= & E^{2} A(0)\left\|x_{a}^{*}-x_{b}^{*}\right\|^{2}\left\{t^{2}+2 t \frac{\left(x_{a}(0)-x_{b}(0)\right)^{\dagger}\left(x_{a}^{*}-x_{b}^{*}\right)}{\sqrt{A(0)}\left\|x_{a}^{*}-x_{b}^{*}\right\|^{2}}+\frac{\left\|x_{a}(0)-x_{b}(0)\right\|^{2}}{A(0)\left\|x_{a}^{*}-x_{b}^{*}\right\|^{2}}\right\} .
\end{aligned}
$$

It was noted above that $E$ tends to 0 like $1 / \sqrt{A(0)} t$; in fact, $E$ is eventually monotone decreasing, as can be seen from

$$
\dot{E}=\frac{1}{1-R(0)^{2}}\left(-\frac{d}{d t} R^{2}\right)=-\frac{2}{1-R(0)^{2}} \frac{\left(1-R^{2}\right) h}{1+R^{2}}=-\frac{2\left(1-R^{2}\right)}{1-R(0)^{2}} \frac{h(0)+t A(0)}{1+R^{2}}=-2 E \frac{h(0)+t A(0)}{1+R^{2}},
$$

which is negative provided $t>-h(0) / A(0)$. We can also show that $\left\|x_{a}-x_{b}\right\|^{2}$ is eventually monotone decreasing, since

$$
\begin{aligned}
\frac{d}{d t}\left\|x_{a}-x_{b}\right\|^{2}=2 E & \dot{E}\left\{t^{2}+2 t \frac{\left(x_{a}(0)-x_{b}(0)\right)^{\dagger}\left(x_{a}^{*}-x_{b}^{*}\right)}{\sqrt{A(0)}\left\|x_{a}^{*}-x_{b}^{*}\right\|^{2}}+\frac{\left\|x_{a}(0)-x_{b}(0)\right\|^{2}}{A(0)\left\|x_{a}^{*}-x_{b}^{*}\right\|^{2}}\right\} \\
+ & 2 E^{2}\left\{t+\frac{\left(x_{a}(0)-x_{b}(0)\right)^{\dagger}\left(x_{a}^{*}-x_{b}^{*}\right)}{\sqrt{A(0)}\left\|x_{a}^{*}-x_{b}^{*}\right\|^{2}}\right\},
\end{aligned}
$$

which is $2 E^{2}$ times

$$
-2 \frac{h(0)+t A(0)}{1+R^{2}}\left\{t^{2}+2 t \frac{\left(x_{a}(0)-x_{b}(0)\right)^{\dagger}\left(x_{a}^{*}-x_{b}^{*}\right)}{\sqrt{A(0)}\left\|x_{a}^{*}-x_{b}^{*}\right\|^{2}}+\frac{\left\|x_{a}(0)-x_{b}(0)\right\|^{2}}{A(0)\left\|x_{a}^{*}-x_{b}^{*}\right\|^{2}}\right\}+t+\frac{\left(x_{a}(0)-x_{b}(0)\right)^{\dagger}\left(x_{a}^{*}-x_{b}^{*}\right)}{\sqrt{A(0)}\left\|x_{a}^{*}-x_{b}^{*}\right\|^{2}},
$$

and this is eventually negative. Thus there is a finite $t_{1}$, such that every particle separation is monotone decreasing on $\left(t_{1}, \infty\right)$. Since the limit point is not a collision point, all the particle separations must be positive, and so must the infimum of the particle separations for, which is equal to

$$
\min _{a \neq b}\left\|x_{a}^{*}-x_{b}^{*}\right\| \text {. }
$$

We summarize most of this Section in the Lemma, wherein we have shifted the initial time from $t=0$ to $t=t_{0}$ :

Lemma 26. Provided the point $\xi$ in the interior of the unit ball has no velocity difference anti-parallel to the corresponding position difference, the system (3.2.2) has a unique solution $\bar{x}(t)$ given in closed form in (3.3.12) for all $t \in\left[t_{0}, \infty\right)$, with $\bar{x}\left(t_{0}\right)=\xi$. Moreover, $\bar{x}$ has a power series expansion in $1 / t$, its first few terms are given in (3.3.18), the series converges for sufficiently large $t$, and $\bar{x}$ tends to a point on the boundary

$$
\left(\begin{array}{c}
x^{*} \\
0_{3 N}
\end{array}\right)=\lim _{t \rightarrow \infty} \bar{x}(t)=\frac{1}{\sqrt{A\left(t_{0}\right)}}\left(\begin{array}{cc}
O_{3 N} & I_{3 N} \\
O_{3 N} & O_{3 N}
\end{array}\right) \bar{x}\left(t_{0}\right)
$$

If in addition, none of the initial velocities are equal, then the limit point is a non-collision point, and the infimum of the particle separations is positive.

The approximate solutions $\tilde{x}$ and $\bar{x}$ are related to the boundary solution as follows:

Proposition 27. Let $\tilde{x}(t)$ be as in (3.3.3), a solution to (3.3.2) starting at $\tilde{x}(0)$. Let $p(t)$ be the unit vector in the direction $\tilde{x}(t)$. Then $p$ is the solution to (3.2.1) starting at $p(0)=\tilde{x}(0) /\|\tilde{x}(0)\|$. Similarly let $\bar{x}(t)$ be as in (3.3.12), a solution to (3.3.11) starting at $\bar{x}(0)$. Let $p(t)$ be the unit vector in the direction $\bar{x}(t)$. Then $p$ is the solution to (3.2.1) starting at $p(0)=\bar{x}(0) /\|\bar{x}(0)\|$.

Proof. For any trajectory $z$, let $h_{z}$ denote the function $h=\sum_{i=1}^{N} z_{i}^{\dagger} z_{N+i}$ along $z$. Let $R=\|\tilde{x}\|$; then from the derivative of $R^{2}$ given in (3.3.5) we have

$$
\dot{R}=\frac{1}{2} \frac{1}{R} 2\left(1-R^{2}\right) h_{\tilde{x}}=\frac{h_{\tilde{x}}}{R}\left(1-R^{2}\right) .
$$




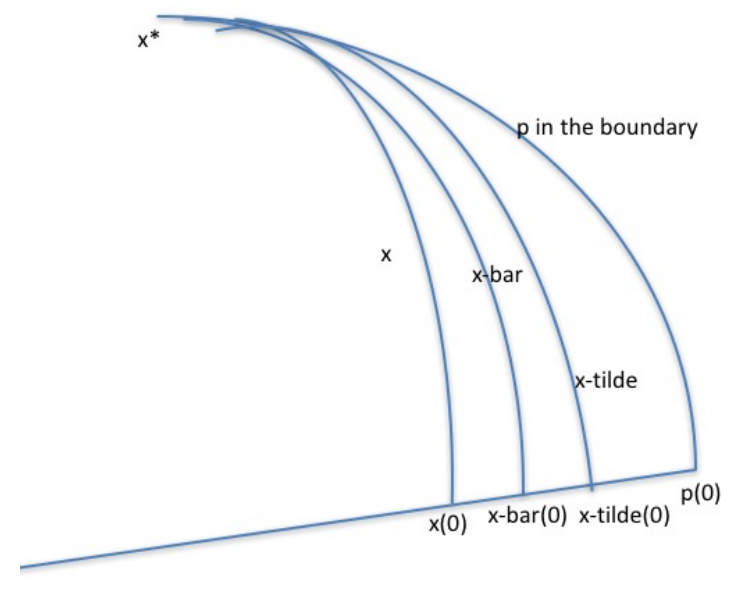

FiguRE 3.3.1. Illustrating Proposition 27

Let $p=\tilde{x} / R$. Then

$$
\begin{aligned}
\dot{p}=\frac{\dot{\tilde{x}}}{R}-\frac{\dot{R} \tilde{x}}{R^{2}} & =-\frac{h_{\tilde{x}}}{R} \tilde{x}+\frac{1}{R}\left(\begin{array}{cc}
O_{3 N} & I_{3 N} \\
O_{3 N} & O_{3 N}
\end{array}\right) \tilde{x}-\frac{h_{\tilde{x}}}{R^{3}}\left(1-R^{2}\right) \tilde{x} \\
& =\frac{1}{R}\left(\begin{array}{cc}
O_{3 N} & I_{3 N} \\
O_{3 N} & O_{3 N}
\end{array}\right) \tilde{x}-\frac{h_{\tilde{x}}}{R}\left(1+\frac{1-R^{2}}{R^{2}}\right) \tilde{x}=\frac{1}{R}\left(\begin{array}{cc}
O_{3 N} & I_{3 N} \\
O_{3 N} & O_{3 N}
\end{array}\right) \tilde{x}-\frac{h_{\tilde{x}}}{R^{3}} \tilde{x} \\
& =-h_{p} p+\left(\begin{array}{cc}
O_{3 N} & I_{3 N} \\
O_{3 N} & O_{3 N}
\end{array}\right) p,
\end{aligned}
$$

which is (3.2.1).

Similarly, let $R=\|\bar{x}\|$, then $p=\bar{x} / R$. From the derivative of $R^{2}$ given in (3.3.14) we have

$$
\dot{R}=\frac{1}{2} \frac{1}{R} 2 \frac{1-R^{2}}{1+R^{2}} h_{\bar{x}}=\frac{h_{\bar{x}}}{R} \frac{1-R^{2}}{1+R^{2}} .
$$

Then

$$
\begin{aligned}
\dot{p}=\frac{\dot{\bar{x}}}{R}-\frac{\dot{R} \bar{x}}{R^{2}} & =\frac{1}{R}\left(-\frac{2 h_{\bar{x}}}{1+R^{2}}+\left(\begin{array}{cc}
O_{3 N} & I_{3 N} \\
O_{3 N} & O_{3 N}
\end{array}\right) \bar{x}\right)-\frac{1-R^{2}}{1+R^{2}} \frac{h_{\bar{x}}}{R^{3}} \bar{x} \\
& =-\frac{1}{R} \frac{h_{\bar{x}}}{1+R^{2}}\left[2+\frac{1-R^{2}}{R^{2}}\right] \bar{x}+\frac{1}{R}\left(\begin{array}{cc}
O_{3 N} & I_{3 N} \\
O_{3 N} & O_{3 N}
\end{array}\right) \bar{x} \\
& =-\frac{h_{\bar{x}}}{R^{3}}+\left(\begin{array}{cc}
O_{3 N} & I_{3 N} \\
O_{3 N} & O_{3 N}
\end{array}\right) p=-h_{p} p+\left(\begin{array}{cc}
O_{3 N} & I_{3 N} \\
O_{3 N} & O_{3 N}
\end{array}\right) p
\end{aligned}
$$

which is (3.2.1).

Examples of the trajectories $x, \bar{x}, \tilde{x}$, and $p$ are shown in Figure 3.3.1.

By contrast, if $x$ is a trajectory in the ball; that is, a solution to the compactified system (3.1.7), let $R=\|x\|$, and $p=x / R$,

$$
\begin{gathered}
\dot{p}=\frac{\dot{x}}{R}-\dot{R} \frac{x}{R^{2}}=\frac{1}{R}\left(\left(\begin{array}{cc}
\theta I_{3 N} & I_{3 N} \\
O_{3 N} & \theta I_{3 N}
\end{array}\right) x+\left(\begin{array}{c}
0_{3 N} \\
\left(1-R^{2}\right)^{3} G
\end{array}\right)\right)+\frac{\left(1-R^{2}\right) \theta}{2 R} \frac{x}{R^{2}} \\
=\left(\begin{array}{cc}
\theta I_{3 N} & I_{3 N} \\
O_{3 N} & \theta I_{3 N}
\end{array}\right) p+\frac{1}{R}\left(\begin{array}{c}
0_{3 N} \\
\left(1-R^{2}\right)^{3} G
\end{array}\right)+\frac{\left(1-R^{2}\right) \theta}{2 R^{2}} p
\end{gathered}
$$




$$
\begin{gathered}
=\left(\begin{array}{ll}
O_{3 N} & I_{3 N} \\
O_{3 N} & O_{3 N}
\end{array}\right) p+\left[1+\frac{1-R^{2}}{2 R^{2}}\right] \theta p+\frac{1}{R}\left(\begin{array}{c}
0_{3 N} \\
\left(1-R^{2}\right)^{3} G
\end{array}\right) \\
=\left(\begin{array}{cc}
O_{3 N} & I_{3 N} \\
O_{3 N} & O_{3 N}
\end{array}\right) p+\frac{1+R^{2}}{2 R^{2}}\left(-2 \frac{h_{x}+\left(1-R^{2}\right)^{3} L}{1+R^{2}}\right) p+\frac{1}{R}\left(\begin{array}{c}
0_{3 N} \\
\left(1-R^{2}\right)^{3} G
\end{array}\right) \\
=\left(\begin{array}{cc}
O_{3 N} & I_{3 N} \\
O_{3 N} & O_{3 N}
\end{array}\right) p-\left(\frac{h_{x}+\left(1-R^{2}\right)^{3} L}{R^{2}}\right) p+\frac{1}{R}\left(\begin{array}{c}
0_{3 N} \\
\left(1-R^{2}\right)^{3} G
\end{array}\right) \\
=\left(\begin{array}{cc}
-h_{p} I_{3 N} & I_{3 N} \\
O_{3 N} & -h_{p} I_{3 N}
\end{array}\right) p-\frac{\left(1-R^{2}\right)^{3} L}{R^{2}} p+\frac{1}{R}\left(\begin{array}{c}
0_{3 N} \\
\left(1-R^{2}\right)^{3} G
\end{array}\right),
\end{gathered}
$$

which is $(3.2 .1)+O\left(\left(1-R^{2}\right)^{3}\right)$.

Also note that if $z$ is any of the above trajectories $\bar{x}, \tilde{x}$, or $x, R=\|z\|$, and $p=z / R$, then

$$
\|z-p\|^{2}=(z-p)^{\dagger}(z-p)=R^{2}-p^{\dagger} z-z^{\dagger} p+1=R^{2}-\frac{2}{R} z^{\dagger} z+1=1-2 R+R^{2}=(1-R)^{2} .
$$

If $z$ is $\bar{x}$ or $\tilde{x}$, or if it is the compactification $x$ of a trajectory $q$ that escapes to $\infty$, this this quantity decays to 0 , giving a further sense in which trajectories in the boundary attract nearby interior trajectories. 


\section{CHAPTER 4}

\section{Seeking Trajectories that Expand to the Boundary}

Given a solution $x$ and an approximation $\bar{x}$ from the previous Chapter, we develop in Section 4.1 the differential equation satisfied by the difference $\Delta=x-\bar{x}$. This requires expressing the right hand side of (3.1.7) in terms of $\Delta$ and the known $\bar{x}$. We will be interested in decaying solutions $\Delta$, whose existence guarantees that $x$ and $\bar{x}$ are asymptotic to the same point $p=\left(x^{* \dagger} 0_{3 N}^{\dagger}\right)^{\dagger}$ as $t \rightarrow \infty$, and we can interpret the unit $3 N$-vector $x^{*}$ as the $N$ limiting directions $x_{i}^{*}$ of the particles. We expand all quantities in $\dot{x}$ as a series in powers of the components of $\Delta$, and compute all terms through $O\left(\|\Delta\|^{2}\right)$. We then establish a series expansion in $1 / t$ for the linear portion and compute all terms through $O\left(1 / t^{2}\right)$. To better understand the problem for $\Delta$, which has an irregular singular point at $t=\infty$, we begin in Section 4.2 with efforts to solve approximating versions of the problem, seeking solutions to the linear problem that decay to 0 . After a very helpful coordinate transformation, we solve the $J_{0}$ problem twice, solve the $J_{-}$problem, and solve the $J_{1}$ problem by finding a convergent series solution tending to 0 . This work with the linear system inspires our search for solutions to the full problem in the next Chapter. We explain in Section 4.3 the motivation for returning to the uncompactified problem and the selection of a specific form of solution to pursue in the next Chapter.

\subsection{Perturbation near an approximate solution}

Given a solution $x$ and an approximation $\bar{x}$, we develop in this Section the differential equation satisfied by the difference $\Delta=x-\bar{x}$. This requires expressing the right hand side of (3.1.7) in terms of $\Delta$ and the known $\bar{x}$. We expand all necessary quantities in a series in powers of the components of $\Delta$, and compute all terms through $O\left(\|\Delta\|^{2}\right)$. We then establish a series expansion in $1 / t$ for the linear portion and compute all terms through $O\left(1 / t^{2}\right)$.

Let $x(t)$ be a solution to the compactified system (3.1.7) through $x_{0}=x\left(t_{0}\right)$, and let $\bar{x}(t)$ be given by (3.3.12), a solution to (3.2.2) starting at $\bar{x}\left(t_{0}\right)$, which is not assumed to be the same point as $x\left(t_{0}\right)$. We assume that $\bar{x}$ does not experience a collision for $t \geq t_{0}$ and does not approach a collision as $t \rightarrow \infty$. Recall from Lemma 26 that this requires of the initial conditions that no initial velocity difference is anti-parallel to the corresponding initial position difference and that no initial velocities are equal. Then by definition, $\Delta=x-\bar{x}$ satisfies the differential equation

$$
\dot{\Delta}=\dot{x}-\dot{\bar{x}}=\frac{-2 h}{1+R^{2}} x+\frac{2 \bar{h}}{1+\bar{R}^{2}} \bar{x}+\left(\begin{array}{cc}
O_{3 N} & I_{3 N} \\
O_{3 N} & O_{3 N}
\end{array}\right) \Delta-\frac{2\left(1-R^{2}\right)^{3} L}{1+R^{2}} x+\left(1-R^{2}\right)^{3}\left(\begin{array}{c}
0_{3 N} \\
G
\end{array}\right)
$$

with initial condition $\Delta\left(t_{0}\right)=x\left(t_{0}\right)-\bar{x}\left(t_{0}\right)$ on the interval $\left[t_{0}, \sigma\right)$, where $\sigma \leq \infty$ is the endpoint of the maximal interval of existence of $x$ per Section 1.1, and

$$
\bar{h}=\sum_{i=1}^{N} \bar{x}_{i}^{\dagger} \bar{x}_{N+i} \quad \bar{R}^{2}=\sum_{i=1}^{2 N} \bar{x}_{i}^{\dagger} \bar{x}_{i}
$$

are the corresponding quantities for $\bar{x}$, and $G$ is the $3 N$-vector composed of the $G_{i}$

$$
G=\left(\begin{array}{c}
G_{1} \\
\vdots \\
G_{N}
\end{array}\right)
$$

Our goal is to establish conditions on $x\left(t_{0}\right)$ and $\bar{x}\left(t_{0}\right)$ so that (4.1.1) will have a solution $\Delta$, which decays to 0 . The right-hand side of (4.1.1) is analytic in $1 / t$ and $\Delta$, so we start by expressing the right hand side of (4.1.1) in terms of $\bar{x}$ and $\Delta$, including terms of $O\left(\|\Delta\|^{3}\right)$. Later, we will express $\bar{x}$ in terms of its series 
expansion in $1 / t$. While we don't expect that a solution $\Delta$ will be analytic in $1 / t$ near $\infty$, it will prove fruitful to expand to low order in $\Delta$ assuming any solution $\Delta$ is small and tends to 0 . From

$$
R^{2}=\|\bar{x}+\Delta\|^{2}=\bar{R}^{2}+2 \bar{x}^{\dagger} \Delta+R_{\Delta}^{2}, \quad \text { with } R_{\Delta}=\|\Delta\|,
$$

we can express the reciprocal

$$
\begin{aligned}
\frac{1}{1+R^{2}} & =\frac{1}{1+\bar{R}^{2}} \frac{1}{1+\frac{2 \bar{x}^{\dagger} \Delta+R_{\Delta}^{2}}{1+\bar{R}^{2}}}=\frac{1}{1+\bar{R}^{2}} \sum_{n=0}^{\infty}\left((-1) \frac{2 \bar{x}^{\dagger} \Delta+R_{\Delta}^{2}}{1+\bar{R}^{2}}\right)^{n} \\
& =\frac{1}{1+\bar{R}^{2}}\left(1-\frac{2 \bar{x}^{\dagger} \Delta+R_{\Delta}^{2}}{1+\bar{R}^{2}}+\frac{\left(2 \bar{x}^{\dagger} \Delta\right)^{2}+4 \bar{x}^{\dagger} \Delta R_{\Delta}^{2}}{\left(1+\bar{R}^{2}\right)^{2}}-\left(\frac{2 \bar{x}^{\dagger} \Delta}{1+\bar{R}^{2}}\right)^{3}\right)+O\left(\|\Delta\|^{4}\right) \\
& =\frac{1}{1+\bar{R}^{2}}\left(1-\frac{2 \bar{x}^{\dagger} \Delta}{1+\bar{R}^{2}}-\frac{R_{\Delta}^{2}}{1+\bar{R}^{2}}+\left(\frac{2 \bar{x}^{\dagger} \Delta}{1+\bar{R}^{2}}\right)^{2}+\frac{4 \bar{x}^{\dagger} \Delta R_{\Delta}^{2}}{\left(1+\bar{R}^{2}\right)^{2}}-\left(\frac{2 \bar{x}^{\dagger} \Delta}{1+\bar{R}^{2}}\right)^{3}\right)+O\left(\|\Delta\|^{4}\right) .
\end{aligned}
$$

As shown, the first two terms inside the big parentheses are constant and linear in $\Delta$, the next two are quadratic, and the last two are cubic. The series converges for

$$
\left|\frac{2 \bar{x}^{\dagger} \Delta+R_{\Delta}^{2}}{1+\bar{R}^{2}}\right|<1
$$

which we can guarantee if $R_{\Delta}<\sqrt{2}-1$, because then

$$
\left|\frac{2 \bar{x}^{\dagger} \Delta+R_{\Delta}^{2}}{1+\bar{R}^{2}}\right| \leq\left|2 \bar{x}^{\dagger} \Delta+R_{\Delta}^{2}\right| \leq 2 R_{\Delta}+R_{\Delta}^{2}<2(\sqrt{2}-1)+(\sqrt{2}-1)^{2}=1 .
$$

Since

$$
h=\bar{h}+\bar{x}^{\dagger}\left(\begin{array}{cc}
O_{3 N} & I_{3 N} \\
I_{3 N} & O_{3 N}
\end{array}\right) \Delta+h_{\Delta}, \quad \text { with } h_{\Delta}=\frac{1}{2} \Delta^{\dagger}\left(\begin{array}{cc}
O_{3 N} & I_{3 N} \\
I_{3 N} & O_{3 N}
\end{array}\right) \Delta,
$$

we have the ratio

$$
\begin{aligned}
\frac{h}{1+R^{2}}= & \frac{1}{1+\bar{R}^{2}}\left[\bar{h}+\bar{x}^{\dagger}\left(\begin{array}{cc}
O_{3 N} & I_{3 N} \\
I_{3 N} & O_{3 N}
\end{array}\right) \Delta+h_{\Delta}\right] \sum_{n=0}^{\infty}\left((-1) \frac{2 \bar{x}^{\dagger} \Delta+R_{\Delta}^{2}}{1+\bar{R}^{2}}\right)^{n} \\
= & \frac{1}{1+\bar{R}^{2}}\left[\bar{h}\left(1-\frac{2 \bar{x}^{\dagger} \Delta+R_{\Delta}^{2}}{1+\bar{R}^{2}}+\left(\frac{2 \bar{x}^{\dagger} \Delta}{1+\bar{R}^{2}}\right)^{2}+\frac{4 \bar{x}^{\dagger} \Delta R_{\Delta}^{2}}{\left(1+\bar{R}^{2}\right)^{2}}-\left(\frac{2 \bar{x}^{\dagger} \Delta}{1+\bar{R}^{2}}\right)^{3}\right)\right. \\
& \left.+\bar{x}^{\dagger}\left(\begin{array}{cc}
O_{3 N} & I_{3 N} \\
I_{3 N} & O_{3 N}
\end{array}\right) \Delta\left(1-\frac{2 \bar{x}^{\dagger} \Delta+R_{\Delta}^{2}}{1+\bar{R}^{2}}+\left(\frac{2 \bar{x}^{\dagger} \Delta}{1+\bar{R}^{2}}\right)^{2}\right)+h_{\Delta}\left(1-\frac{2 \bar{x}^{\dagger} \Delta}{1+\bar{R}^{2}}\right)\right]+O\left(\|\Delta\|^{4}\right) .
\end{aligned}
$$

For convenience we arrange by order in $\Delta$

$$
\begin{aligned}
\frac{h}{1+R^{2}}= & \frac{\bar{h}}{1+\bar{R}^{2}}-\frac{2 \bar{h} \bar{x}^{\dagger} \Delta}{\left(1+\bar{R}^{2}\right)^{2}}+\frac{1}{1+\bar{R}^{2}} \bar{x}^{\dagger}\left(\begin{array}{cc}
O_{3 N} & I_{3 N} \\
I_{3 N} & O_{3 N}
\end{array}\right) \Delta \\
& +\frac{\bar{h}\left(2 \bar{x}^{\dagger} \Delta\right)^{2}}{\left(1+\bar{R}^{2}\right)^{3}}-\frac{\bar{h} R_{\Delta}^{2}}{\left(1+\bar{R}^{2}\right)^{2}}-\bar{x}^{\dagger}\left(\begin{array}{cc}
O_{3 N} & I_{3 N} \\
I_{3 N} & O_{3 N}
\end{array}\right) \Delta \frac{2 \bar{x}^{\dagger} \Delta}{\left(1+\bar{R}^{2}\right)^{2}}+\frac{h_{\Delta}}{1+\bar{R}^{2}} \\
& +\frac{4 \bar{h} \bar{x}^{\dagger} \Delta R_{\Delta}^{2}}{\left(1+\bar{R}^{2}\right)^{3}}-\frac{\bar{h}\left(2 \bar{x}^{\dagger} \Delta\right)^{3}}{\left(1+\bar{R}^{2}\right)^{4}}+\bar{x}^{\dagger}\left(\begin{array}{cc}
O_{3 N} & I_{3 N} \\
I_{3 N} & O_{3 N}
\end{array}\right) \Delta\left(\frac{\left(2 \bar{x}^{\dagger} \Delta\right)^{2}}{\left(1+\bar{R}^{2}\right)^{3}}-\frac{R_{\Delta}^{2}}{\left(1+\bar{R}^{2}\right)^{2}}\right)-\frac{2 h_{\Delta} \bar{x}^{\dagger} \Delta}{\left(1+\bar{R}^{2}\right)^{2}} \\
& +O\left(\|\Delta\|^{4}\right) .
\end{aligned}
$$


Now we can form $\frac{h}{1+R^{2}} x$, which is the above times $\bar{x}+\Delta$

$$
\begin{aligned}
& \frac{h}{1+R^{2}} x=\frac{\bar{h}}{1+\bar{R}^{2}} \bar{x}-\frac{2 \bar{h} \bar{x}^{\dagger} \Delta}{\left(1+\bar{R}^{2}\right)^{2}} \bar{x}+\frac{1}{1+\bar{R}^{2}} \bar{x}^{\dagger}\left(\begin{array}{cc}
O_{3 N} & I_{3 N} \\
I_{3 N} & O_{3 N}
\end{array}\right) \Delta \bar{x}+\frac{\bar{h}}{1+\bar{R}^{2}} \Delta \\
&-\frac{2 \bar{h} \bar{x}^{\dagger} \Delta}{\left(1+\bar{R}^{2}\right)^{2}} \Delta+\frac{1}{1+\bar{R}^{2}} \bar{x}^{\dagger}\left(\begin{array}{cc}
O_{3 N} & I_{3 N} \\
I_{3 N} & O_{3 N}
\end{array}\right) \Delta \Delta \\
&+ \frac{\bar{h}\left(2 \bar{x}^{\dagger} \Delta\right)^{2}}{\left(1+\bar{R}^{2}\right)^{3}} \bar{x}-\frac{\bar{h} R_{\Delta}^{2}}{\left(1+\bar{R}^{2}\right)^{2}} \bar{x}-\bar{x}^{\dagger}\left(\begin{array}{cc}
O_{3 N} & I_{3 N} \\
I_{3 N} & O_{3 N}
\end{array}\right) \Delta \frac{2 \bar{x}^{\dagger} \Delta}{\left(1+\bar{R}^{2}\right)^{2}} \bar{x}+\frac{h_{\Delta}}{1+\bar{R}^{2}} \bar{x} \\
&+ \frac{\bar{h}\left(2 \bar{x}^{\dagger} \Delta\right)^{2}}{\left(1+\bar{R}^{2}\right)^{3}} \Delta-\frac{\bar{h} R_{\Delta}^{2}}{\left(1+\bar{R}^{2}\right)^{2}} \Delta-\bar{x}^{\dagger}\left(\begin{array}{cc}
O_{3 N} & I_{3 N} \\
I_{3 N} & O_{3 N}
\end{array}\right) \Delta \frac{2 \bar{x}^{\dagger} \Delta}{\left(1+\bar{R}^{2}\right)^{2}} \Delta+\frac{h_{\Delta}}{1+\bar{R}^{2}} \Delta \\
&+ \frac{4 \bar{h} \bar{x}^{\dagger} \Delta R_{\Delta}^{2}}{\left(1+\bar{R}^{2}\right)^{3}} \bar{x}-\frac{\bar{h}\left(2 \bar{x}^{\dagger} \Delta\right)^{3}}{\left(1+\bar{R}^{2}\right)^{4}} \bar{x}+\bar{x}^{\dagger}\left(\begin{array}{cc}
O_{3 N} & I_{3 N} \\
I_{3 N} & O_{3 N}
\end{array}\right) \Delta\left(\frac{\left(2 \bar{x}^{\dagger} \Delta\right)^{2}}{\left(1+\bar{R}^{2}\right)^{3}}-\frac{R_{\Delta}^{2}}{\left(1+\bar{R}^{2}\right)^{2}}\right) \bar{x} \\
&-\frac{2 h_{\Delta} \bar{x}^{\dagger} \Delta}{\left(1+\bar{R}^{2}\right)^{2}} \bar{x}+O\left(\|\Delta\|^{4}\right) .
\end{aligned}
$$

Then the difference

$$
\begin{aligned}
h x-\bar{h} \bar{x}=\bar{h} \Delta & -\bar{h} \frac{2 \bar{x}^{\dagger} \Delta}{1+\bar{R}^{2}} \bar{x}+\bar{x}^{\dagger}\left(\begin{array}{cc}
O_{3 N} & I_{3 N} \\
I_{3 N} & O_{3 N}
\end{array}\right) \Delta \bar{x}-\bar{h} \frac{2 \bar{x}^{\dagger} \Delta}{1+\bar{R}^{2}} \Delta-\bar{h} \frac{R_{\Delta}^{2}}{1+\bar{R}^{2}} \bar{x}+\bar{h}\left(\frac{2 \bar{x}^{\dagger} \Delta}{1+\bar{R}^{2}}\right)^{2} \bar{x} \\
& +\bar{x}^{\dagger}\left(\begin{array}{cc}
O_{3 N} & I_{3 N} \\
I_{3 N} & O_{3 N}
\end{array}\right) \Delta\left(\Delta-\frac{2 \bar{x}^{\dagger} \Delta}{1+\bar{R} \bar{x}^{2}} \bar{x}\right)+h_{\Delta} \bar{x} \\
& +\frac{\bar{h}\left(2 \bar{x}^{\dagger} \Delta\right)^{2}}{\left(1+\bar{R}^{2}\right)^{2}} \Delta-\frac{\bar{h} R_{\Delta}^{2}}{1+\bar{R}^{2}} \Delta-\bar{x}^{\dagger}\left(\begin{array}{cc}
O_{3 N} & I_{3 N} \\
I_{3 N} & O_{3 N}
\end{array}\right) \Delta \frac{2 \bar{x}^{\dagger} \Delta}{1+\bar{R}^{2}} \Delta+h_{\Delta} \Delta \\
& +\frac{4 \bar{h} \bar{x}^{\dagger} \Delta R_{\Delta}^{2}}{\left(1+\bar{R}^{2}\right)^{2}}-\frac{\bar{h}\left(2 \bar{x}^{\dagger} \Delta\right)^{3}}{\left(1+\bar{R}^{2}\right)^{3}} \bar{x}+\bar{x}^{\dagger}\left(\begin{array}{cc}
O_{3 N} & I_{3 N} \\
I_{3 N} & O_{3 N}
\end{array}\right) \Delta\left(\frac{\left(2 \bar{x}^{\dagger} \Delta\right)^{2}}{\left(1+\bar{R}^{2}\right)^{2}}-\frac{R_{\Delta}^{2}}{1+\bar{R}^{2}}\right) \bar{x} \\
& -\frac{2 h_{\Delta} \bar{x}^{\dagger} \Delta}{1+\bar{R}^{2}} \bar{x}+O\left(\|\Delta\|^{4}\right) .
\end{aligned}
$$

Since $G_{i}$ and $L$ appear multiplied by $\left(1-R^{2}\right)^{3}$ and since $1-R^{2}=O(1 / t)$, we will only keep terms of $G_{i}$ and $L$ to $O\left(\|\Delta\|^{2}\right)$. We start with $G_{i}$, and first we need

$$
\begin{aligned}
x_{k}-x_{i} & =\bar{x}_{k}-\bar{x}_{i}+\Delta_{k}-\Delta_{i} \\
g_{i k}^{2} & =\bar{g}_{i k}^{2}\left(1+\frac{1}{\bar{g}_{i k}^{2}}\left[2\left(\bar{x}_{k}-\bar{x}_{i}\right)^{\dagger}\left(\Delta_{k}-\Delta_{i}\right)+\left\|\Delta_{k}-\Delta_{i}\right\|^{2}\right]\right) .
\end{aligned}
$$

Note from Lemma 26 that for sufficiently large $t_{1}$, the $\bar{g}_{i k}$ are bounded away from 0 on $t \geq t_{1}$, so this is meaningful. Let $\delta$ be the minimum separation distance of the $\bar{x}_{j}$, which is $\min _{a \neq b}\left\|x_{a}^{*}-x_{b}^{*}\right\|$. Then the set of reciprocals $\left\{\bar{g}_{i k}^{-1}\right\}$ is uniformly bounded by $1 / \delta$. We need $g_{i k}^{-3}$, which we will expand in a series, convergent if

$$
\frac{1}{\bar{g}_{i k}^{2}}\left[2\left(\bar{x}_{k}-\bar{x}_{i}\right)^{\dagger}\left(\Delta_{k}-\Delta_{i}\right)+\left\|\Delta_{k}-\Delta_{i}\right\|^{2}\right]<1
$$

Assuming that the solution $\Delta$ goes to 0 , we know that for any $\epsilon>0$, there is a time $t_{2}$, such that $\|\Delta(t)\|<\epsilon$, for $t>t_{2}$. Let us choose $\epsilon<\frac{\sqrt{2}-1}{2} \delta$, and restrict our attention to times $t \geq t_{3}=\max \left\{t_{1}, t_{2}\right\}$. Then

$$
\begin{aligned}
\frac{1}{\bar{g}_{i k}^{2}}\left[2\left(\bar{x}_{k}-\bar{x}_{i}\right)^{\dagger}\left(\Delta_{k}-\Delta_{i}\right)\right. & \left.+\left\|\Delta_{k}-\Delta_{i}\right\|^{2}\right] \leq \frac{2\left\|\Delta_{k}-\Delta_{i}\right\|}{\bar{g}_{i k}}+\frac{\left\|\Delta_{k}-\Delta_{i}\right\|^{2}}{\bar{g}_{i k}^{2}} \leq \frac{4 \epsilon}{\delta}+\frac{4 \epsilon^{2}}{\delta^{2}} \\
& <2(\sqrt{2}-1)+(\sqrt{2}-1)^{2}=1,
\end{aligned}
$$


and the series for $g_{i k}^{2}$ converges for $t \geq t_{3}$. Keeping terms of second order in $\Delta$, we can expand as $g_{i k}^{-3}=\bar{g}_{i k}^{-3}\left(1-\frac{3}{2 \bar{g}_{i k}^{2}}\left[2\left(\bar{x}_{k}-\bar{x}_{i}\right)^{\dagger}\left(\Delta_{k}-\Delta_{i}\right)+\left\|\Delta_{k}-\Delta_{i}\right\|^{2}\right]+\frac{15}{8 \bar{g}_{i k}^{4}}\left[2\left(\bar{x}_{k}-\bar{x}_{i}\right)^{\dagger}\left(\Delta_{k}-\Delta_{i}\right)\right]^{2}\right)+O\left(\|\Delta\|^{3}\right)$.

As a linear combination of terms of the form

$$
\begin{aligned}
\frac{x_{k}-x_{i}}{g_{i k}^{3}}= & \frac{\bar{x}_{k}-\bar{x}_{i}+\Delta_{k}-\Delta_{i}}{\bar{g}_{i k}^{3}}-\frac{3}{\bar{g}_{i k}^{5}}\left(\bar{x}_{k}-\bar{x}_{i}\right)^{\dagger}\left(\Delta_{k}-\Delta_{i}\right)\left(\bar{x}_{k}-\bar{x}_{i}\right) \\
& +\frac{15}{2 \bar{g}_{i k}^{7}}\left[\left(\bar{x}_{k}-\bar{x}_{i}\right)^{\dagger}\left(\Delta_{k}-\Delta_{i}\right)\right]^{2}\left(\bar{x}_{k}-\bar{x}_{i}\right) \\
& -\frac{3}{2 \bar{g}_{i k}^{5}}\left[2\left(\bar{x}_{k}-\bar{x}_{i}\right)^{\dagger}\left(\Delta_{k}-\Delta_{i}\right)\left(\Delta_{k}-\Delta_{i}\right)+\left\|\Delta_{k}-\Delta_{i}\right\|^{2}\left(\bar{x}_{k}-\bar{x}_{i}\right)\right]+O\left(\|\Delta\|^{3}\right),
\end{aligned}
$$

each $G_{i}$ has a series in $\Delta$, convergent for $t \geq t_{3}$. Collecting terms by powers of $\Delta$, we have the first few terms

$$
G_{i}=\bar{G}_{i}+G_{1 i}(\Delta)+G_{2 i}(\Delta)+O\left(\|\Delta\|^{3}\right)
$$

with

$$
\begin{aligned}
\bar{G}_{i}= & \sum_{k \neq i} \frac{m_{k}}{\bar{g}_{i k}^{3}}\left(\bar{x}_{k}-\bar{x}_{i}\right) \\
G_{1 i}(\Delta)= & \sum_{k \neq i} \frac{m_{k}}{\bar{g}_{i k}^{3}}\left[I_{3}-\frac{3}{\bar{g}_{i k}^{2}}\left(\bar{x}_{k}-\bar{x}_{i}\right)\left(\bar{x}_{k}-\bar{x}_{i}\right)^{\dagger}\right]\left(\Delta_{k}-\Delta_{i}\right) \\
G_{2 i}(\Delta)= & \frac{15}{2} \sum_{k \neq i} \frac{m_{k}}{\bar{g}_{i k}^{7}}\left[\left(\bar{x}_{k}-\bar{x}_{i}\right)^{\dagger}\left(\Delta_{k}-\Delta_{i}\right)\right]^{2}\left(\bar{x}_{k}-\bar{x}_{i}\right) \\
& \quad-\frac{3}{2} \sum_{k \neq i} \frac{m_{k}}{\bar{g}_{i k}^{5}}\left[2\left(\bar{x}_{k}-\bar{x}_{i}\right)^{\dagger}\left(\Delta_{k}-\Delta_{i}\right)\left(\Delta_{k}-\Delta_{i}\right)+\left\|\Delta_{k}-\Delta_{i}\right\|^{2}\left(\bar{x}_{k}-\bar{x}_{i}\right)\right] .
\end{aligned}
$$

Recall that $I_{3}$ is the $3 \times 3$ identity matrix, and note that for each $i \neq k,\left(\bar{x}_{k}-\bar{x}_{i}\right)\left(\bar{x}_{k}-\bar{x}_{i}\right)^{\dagger}$ is a $3 \times 3$ matrix.

For the contribution from the terms of (4.1.1) cubic in $1-R^{2}$, we need

$$
\left(1-R^{2}\right)^{3}=\left(1-\bar{R}^{2}\right)^{3}-3\left(1-\bar{R}^{2}\right)^{2}\left(2 \bar{x}^{\dagger} \Delta+R_{\Delta}^{2}\right)+3\left(1-\bar{R}^{2}\right)\left(2 \bar{x}^{\dagger} \Delta\right)^{2}+O\left(\|\Delta\|^{3}\right) .
$$

Now we can put the pieces together,

$$
\begin{aligned}
\left(1-R^{2}\right)^{3} G_{i}= & \left(1-\bar{R}^{2}\right)^{3}\left(\bar{G}_{i}+G_{1 i}(\Delta)+G_{2 i}(\Delta)\right)-6\left(1-\bar{R}^{2}\right)^{2} \bar{x}^{\dagger} \Delta\left(\bar{G}_{i}+G_{1 i}(\Delta)\right) \\
& -3\left(1-\bar{R}^{2}\right)^{2} R_{\Delta}^{2} \bar{G}_{i}+3\left(1-\bar{R}^{2}\right)\left(2 \bar{x}^{\dagger} \Delta\right)^{2} \bar{G}_{i}+O\left(\|\Delta\|^{3}\right),
\end{aligned}
$$

which we can rearrange:

$$
\begin{aligned}
\left(1-R^{2}\right)^{3} G_{i}=( & \left.1-\bar{R}^{2}\right)^{3} \bar{G}_{i}-6\left(1-\bar{R}^{2}\right)^{2} \bar{x}^{\dagger} \Delta \bar{G}_{i}-6\left(1-\bar{R}^{2}\right)^{2} \bar{x}^{\dagger} \Delta G_{1 i}(\Delta) \\
& -3\left(1-\bar{R}^{2}\right)^{2} R_{\Delta}^{2} \bar{G}_{i}+12\left(1-\bar{R}^{2}\right)\left(\bar{x}^{\dagger} \Delta\right)^{2} \bar{G}_{i} \\
& +\left(1-\bar{R}^{2}\right)^{3} G_{1 i}(\Delta)+\left(1-\bar{R}^{2}\right)^{3} G_{2 i}(\Delta)+O\left(\|\Delta\|^{3}\right)
\end{aligned}
$$

The last two terms above are linear or quadratic in $\|\Delta\|$, which is decreasing, and $O\left(1 / t^{3}\right)$, since $1-\bar{R}^{2}=$ $O(1 / t)$, and they will be neglected going forward. We will see that the first term in (4.1.5) will contribute to the non-homogeneous term; the next term is linear in $\Delta$, and the rest are quadratic terms.

Based on the expansion of $G_{i}$ above, we express $L$ through second order in $\|\Delta\|$ :

$$
L=\sum_{k=1}^{N}\left(\bar{x}_{N+k}+\Delta_{N+k}\right)^{\dagger}\left(\bar{G}_{k}+G_{1 k}(\Delta)+G_{2 k}(\Delta)\right)=\bar{L}+L_{1}(\Delta)+L_{2}(\Delta)+O\left(\|\Delta\|^{3}\right),
$$


the first few terms of its power series, convergent for $t \geq t_{3}$, where

$$
\begin{aligned}
\bar{L} & =\sum_{k=1}^{N} \bar{x}_{N+k}^{\dagger} \bar{G}_{k} \\
L_{1}(\Delta) & =\sum_{k=1}^{N} \Delta_{N+k}^{\dagger} \bar{G}_{k}+\sum_{k=1}^{N} \bar{x}_{N+k}^{\dagger} G_{1 k}(\Delta) \\
L_{2}(\Delta) & =\sum_{k=1}^{N} \Delta_{N+k}^{\dagger} G_{1 k}(\Delta)+\sum_{k=1}^{N} \bar{x}_{N+k}^{\dagger} G_{2 k}(\Delta) .
\end{aligned}
$$

Combining (4.1.2) and (4.1.4) we have

$$
\begin{aligned}
\frac{\left(1-R^{2}\right)^{3}}{1+R^{2}}= & \frac{1-\frac{2 \bar{x}^{\dagger} \Delta+R_{\Delta}^{2}}{1+\bar{R}^{2}}+\left(\frac{2 \bar{x}^{\dagger} \Delta}{1+\bar{R}^{2}}\right)^{2}}{1+\bar{R}^{2}} \\
& \times\left(\left(1-\bar{R}^{2}\right)^{3}-3\left(1-\bar{R}^{2}\right)^{2}\left(2 \bar{x}^{\dagger} \Delta+R_{\Delta}^{2}\right)+3\left(1-\bar{R}^{2}\right)\left(2 \bar{x}^{\dagger} \Delta\right)^{2}\right)+O\left(\|\Delta\|^{3}\right) \\
= & \frac{\left(1-\bar{R}^{2}\right)^{3}}{1+\bar{R}^{2}}-\frac{6\left(1-\bar{R}^{2}\right)^{2}}{1+\bar{R}^{2}} \bar{x}^{\dagger} \Delta-\frac{3\left(1-\bar{R}^{2}\right)^{2}}{1+\bar{R}^{2}} R_{\Delta}^{2}+\frac{12\left(1-\bar{R}^{2}\right)}{1+\bar{R}^{2}}\left(\bar{x}^{\dagger} \Delta\right)^{2} \\
& -\frac{2 \bar{x}^{\dagger} \Delta}{1+\bar{R}^{2}}\left[\frac{\left(1-\bar{R}^{2}\right)^{3}}{1+\bar{R}^{2}}-\frac{6\left(1-\bar{R}^{2}\right)^{2}}{1+\bar{R}^{2}} \bar{x}^{\dagger} \Delta\right] \\
& +\left(\left(\frac{2 \bar{x}^{\dagger} \Delta}{1+\bar{R}^{2}}\right)^{2}-\frac{R_{\Delta}^{2}}{1+\bar{R}^{2}}\right)\left(1-\bar{R}^{2}\right)^{3}+O\left(\|\Delta\|^{3}\right)
\end{aligned}
$$

The terms in $\left(\bar{x}^{\dagger} \Delta\right)^{2}$ can be combined, and multiplying this by $L x$, we remove terms in $\Delta$ which are $O\left(1 / t^{3}\right)$, leaving

$$
\frac{\left(1-R^{2}\right)^{3}}{1+R^{2}}=\frac{\left(1-\bar{R}^{2}\right)^{3}}{1+\bar{R}^{2}}-\frac{6\left(1-\bar{R}^{2}\right)^{2}}{1+\bar{R}^{2}} \bar{x}^{\dagger} \Delta-\frac{3\left(1-\bar{R}^{2}\right)^{2}}{1+\bar{R}^{2}} R_{\Delta}^{2}+\frac{24\left(1-\bar{R}^{2}\right)}{\left(1+\bar{R}^{2}\right)^{2}}\left(\bar{x}^{\dagger} \Delta\right)^{2}
$$

Then we can put the pieces together

$$
L x=\left(\bar{L}+L_{1}(\Delta)+L_{2}(\Delta)\right)(\bar{x}+\Delta)+O\left(\Delta^{3}\right)=\bar{L} \bar{x}+\bar{L} \Delta+L_{1}(\Delta) \bar{x}+L_{1}(\Delta) \Delta+L_{2}(\Delta) \bar{x}+O\left(\|\Delta\|^{3}\right),
$$

so that

$$
\begin{aligned}
\frac{\left(1-R^{2}\right)^{3}}{1+R^{2}} L x= & \left(\frac{\left(1-\bar{R}^{2}\right)^{3}}{1+\bar{R}^{2}}-\frac{6\left(1-\bar{R}^{2}\right)^{2}}{1+\bar{R}^{2}} \bar{x}^{\dagger} \Delta-\frac{3\left(1-\bar{R}^{2}\right)^{2}}{1+\bar{R}^{2}} R_{\Delta}^{2}+\frac{24\left(1-\bar{R}^{2}\right)}{\left(1+\bar{R}^{2}\right)^{2}}\left(\bar{x}^{\dagger} \Delta\right)^{2}\right) \bar{L} \bar{x} \\
& +\left(\frac{\left(1-\bar{R}^{2}\right)^{3}}{1+\bar{R}^{2}}-\frac{6\left(1-\bar{R}^{2}\right)^{2}}{1+\bar{R}^{2}} \bar{x}^{\dagger} \Delta\right)\left[\bar{L} \Delta+L_{1}(\Delta) \bar{x}\right] \\
& +\frac{\left(1-\bar{R}^{2}\right)^{3}}{1+\bar{R}^{2}}\left[L_{1}(\Delta) \Delta+L_{2}(\Delta) \bar{x}\right]+O\left(\|\Delta\|^{3}\right) .
\end{aligned}
$$

Since $\bar{L}=O(1 / t)$, the above contains terms in $\Delta$ which are $O\left(1 / t^{3}\right)$, so we remove them leaving

$$
\frac{\left(1-R^{2}\right)^{3}}{1+R^{2}} L x=\left(\frac{\left(1-\bar{R}^{2}\right)^{3}}{1+\bar{R}^{2}}+\frac{24\left(1-\bar{R}^{2}\right)}{\left(1+\bar{R}^{2}\right)^{2}}\left(\bar{x}^{\dagger} \Delta\right)^{2}\right) \bar{L} \bar{x}-\frac{6\left(1-\bar{R}^{2}\right)^{2}}{1+\bar{R}^{2}} \bar{x}^{\dagger} \Delta L_{1}(\Delta) \bar{x}+O\left(\|\Delta\|^{3}\right)
$$

The first term in (4.1.6) will contribute to the non-homogeneous term below, and the other two are quadratic in $\Delta$. Collecting the terms, we have the linear part and the non-homogeneous part in the first two lines, 
followed by the part quadratic in $\Delta$ :

$$
\begin{aligned}
\dot{\Delta}= & \left(\frac{-2}{1+\bar{R}^{2}}\left[\bar{h}\left(\begin{array}{cc}
I_{3 N} & O_{3 N} \\
O_{3 N} & I_{3 N}
\end{array}\right)-\frac{2 \bar{h}}{1+\bar{R}^{2}} \bar{x} \bar{x}^{\dagger}+\bar{x} \bar{x}^{\dagger}\left(\begin{array}{cc}
O_{3 N} & I_{3 N} \\
I_{3 N} & O_{3 N}
\end{array}\right)\right]+\left(\begin{array}{cc}
O_{3 N} & I_{3 N} \\
O_{3 N} & O_{3 N}
\end{array}\right)\right) \Delta \\
& -6\left(1-\bar{R}^{2}\right)^{2}\left(\begin{array}{c}
0_{3 N} \\
\bar{G}
\end{array}\right) \bar{x}^{\dagger} \Delta-\frac{2\left(1-\bar{R}^{2}\right)^{3}}{1+\bar{R}^{2}} \bar{L} \bar{x}+\left(1-\bar{R}^{2}\right)^{3}\left(\begin{array}{c}
0_{3 N} \\
\bar{G}
\end{array}\right) \\
& +\frac{2}{1+\bar{R}^{2}}\left\{\bar{h}\left[\frac{2 \bar{x}^{\dagger} \Delta}{1+\bar{R}^{2}} \Delta+\frac{R_{\Delta}^{2}}{1+\bar{R}^{2}} \bar{x}-\left(\frac{2 \bar{x}^{\dagger} \Delta}{1+\bar{R}^{2}}\right)^{2} \bar{x}\right]-\frac{48\left(1-\bar{R}^{2}\right)}{1+\bar{R}^{2}}\left(\bar{x}^{\dagger} \Delta\right)^{2} \bar{L} \bar{x}\right. \\
& +\frac{12\left(1-\bar{R}^{2}\right)^{2}}{1+\bar{R}^{2}} \bar{x}^{\dagger} \Delta L_{1}(\Delta) \bar{x}-\bar{x}^{\dagger}\left(\begin{array}{cc}
O_{3 N} & I_{3 N} \\
I_{3 N} & O_{3 N}
\end{array}\right) \Delta \Delta \\
& \left.+\bar{x}^{\dagger}\left(\begin{array}{cc}
O_{3 N} & I_{3 N} \\
I_{3 N} & O_{3 N}
\end{array}\right) \Delta \frac{2 \bar{x}^{\dagger} \Delta}{1+\bar{R}^{2}} \bar{x}-h_{\Delta} \bar{x}\right\} \\
& -6\left(1-\bar{R}^{2}\right)^{2} \bar{x}^{\dagger} \Delta\left(\begin{array}{c}
0_{3 N} \\
G_{1}(\Delta)
\end{array}\right)-3\left(1-\bar{R}^{2}\right)^{2} R_{\Delta}^{2}\left(\begin{array}{c}
O_{3 N} \\
\bar{G}
\end{array}\right) \\
+ & 12\left(1-\bar{R}^{2}\right)\left(\bar{x}^{\dagger} \Delta\right)^{2}\left(\begin{array}{c}
0_{3 N} \\
\bar{G}
\end{array}\right)+O\left(\|\Delta\|^{3}\right) .
\end{aligned}
$$

Note that the linear and quadratic terms are explicitly shown only through $O\left(1 / t^{2}\right)$, as we have removed the linear and quadratic (in $\Delta$ ) terms of $O\left(1 / t^{3}\right)$ and above.

Our stability analysis of (4.1.7) begins with the linear terms, which we express in terms of the expansion of $\bar{x}$ near the critical point $x^{*}$ from (3.3.18):

$$
\bar{x}=\left(\begin{array}{c}
x^{*}+a_{1} / t+a_{2} / t^{2}+a_{3} / t^{3}+\ldots \\
x^{*}\left(1 / t+B_{2} / t^{2}+B_{3} / t^{3}+\ldots\right)
\end{array}\right),
$$

where the $a_{k}$ are $3 N$-vectors and the $B_{k}$ are scalars (since $\bar{x}_{N+i} \propto x_{i}^{*}$ ), and we know $B_{1}=1$. Let $A_{k}=a_{k}^{\dagger} x^{*}$. We are assuming that we can choose the initial time $t_{0}$ to be as large as necessary, so in particular, we we will keep terms through $1 / t^{2}$ in the linear coefficient matrix and the lowest order for each block of the non-homogeneous term.

Lemma 28. The coefficient of the linear term on the right side of (4.1.7) is $J_{2}+O\left(1 / t^{3}\right)$, where

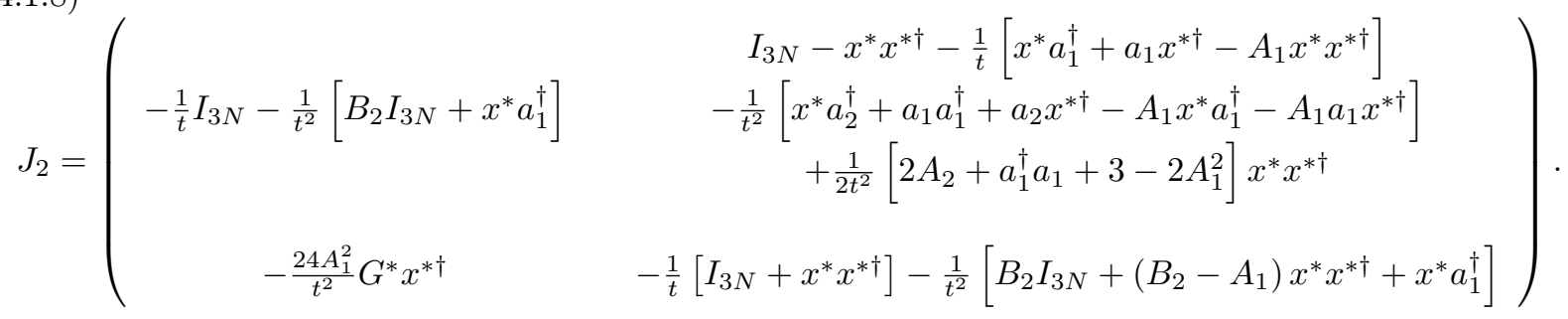

PROOF. For the linear part including all terms of order $1 / t^{2}$, we have

$$
\begin{aligned}
\bar{R}^{2} & =1+\frac{2 A_{1}}{t}+\frac{1}{t^{2}}\left[2 A_{2}+a_{1}^{\dagger} a_{1}+1\right]+O\left(\frac{1}{t^{3}}\right) \\
1-\bar{R}^{2} & =-\frac{2 A_{1}}{t}-\frac{1}{t^{2}}\left[2 A_{2}+a_{1}^{\dagger} a_{1}+1\right]+O\left(\frac{1}{t^{3}}\right), \quad\left(1-\bar{R}^{2}\right)^{2}=\frac{4 A_{1}^{2}}{t^{2}}+O\left(\frac{1}{t^{3}}\right),
\end{aligned}
$$

so we can express

$$
\frac{2}{1+\bar{R}^{2}}=\frac{1}{1-\frac{1}{2}\left(1-\bar{R}^{2}\right)}=1-\frac{A_{1}}{t}-\frac{1}{2 t^{2}}\left[2 A_{2}+a_{1}^{\dagger} a_{1}+1-2 A_{1}^{2}\right]+O\left(\frac{1}{t^{3}}\right) .
$$


Then with

$$
\bar{h}=\frac{1}{t}+\frac{1}{t^{2}}\left[B_{2}+A_{1}\right]+O\left(\frac{1}{t^{3}}\right)
$$

we have

$$
\frac{2 \bar{h}}{1+\bar{R}^{2}}=\frac{1}{t}+\frac{B_{2}}{t^{2}}+O\left(\frac{1}{t^{3}}\right) .
$$

Now we can express the matrix $\bar{x} \bar{x}^{\dagger}$ in terms of the expansion above as

$$
\left(\begin{array}{cc}
x^{*} x^{* \dagger}+\frac{1}{t}\left[x^{*} a_{1}^{\dagger}+a_{1} x^{* \dagger}\right]+\frac{1}{t^{2}}\left[x^{*} a_{2}^{\dagger}+a_{1} a_{1}^{\dagger}+a_{2} x^{* \dagger}\right] & \frac{1}{t} x^{*} x^{* \dagger}+\frac{1}{t^{2}}\left[B_{2} x^{*} x^{* \dagger}+a_{1} x^{* \dagger}\right] \\
\frac{1}{t} x^{*} x^{* \dagger}+\frac{1}{t^{2}}\left[B_{2} x^{*} x^{* \dagger}+x^{*} a_{1}^{\dagger}\right] & \frac{1}{t^{2}} x^{*} x^{* \dagger}
\end{array}\right)+O\left(\frac{1}{t^{3}}\right)
$$

So we can form

$$
\frac{2 \bar{h}}{1+\bar{R}^{2}} \bar{x} \bar{x}^{\dagger}=\left(\begin{array}{cc}
\frac{1}{t} x^{*} x^{* \dagger}+\frac{1}{t^{2}}\left[x^{*} a_{1}^{\dagger}+a_{1} x^{* \dagger}+B_{2} x^{*} x^{* \dagger}\right] & \frac{1}{t^{2}} x^{*} x^{* \dagger} \\
\frac{1}{t^{2}} x^{*} x^{* \dagger} & O_{3 N}
\end{array}\right)+O\left(\frac{1}{t^{3}}\right) .
$$

Note that multiplying on the right by the matrix $\left(\begin{array}{cc}O_{3 N} & I_{3 N} \\ I_{3 N} & O_{3 N}\end{array}\right)$ switches the columns, so we have

$$
\begin{gathered}
\bar{h}\left(\begin{array}{cc}
I_{3 N} & O_{3 N} \\
O_{3 N} & I_{3 N}
\end{array}\right)+\bar{x} \bar{x}^{\dagger}\left(\begin{array}{cc}
O_{3 N} & I_{3 N} \\
I_{3 N} & O_{3 N}
\end{array}\right)-\frac{2 \bar{h}}{1+\bar{R}^{2}} \bar{x} \bar{x}^{\dagger} \\
O_{3 N} \\
\left.\begin{array}{c}
\frac{1}{t} I_{3 N}+\frac{1}{t^{2}}\left[\left(B_{2}+A_{1}\right) I_{3 N}-x^{*} a_{1}^{\dagger}\right] \\
x^{*} x^{* \dagger}+\frac{1}{t}\left[x^{*} a_{1}^{\dagger}+a_{1} x^{* \dagger}\right] \\
+\frac{1}{t^{2}}\left[x^{*} a_{2}^{\dagger}+a_{1} a_{1}^{\dagger}+a_{2} x^{* \dagger}-x^{*} x^{* \dagger}\right] \\
\frac{1}{t}\left[I_{3 N}+x^{*} x^{* \dagger}\right] \\
+\frac{1}{t^{2}}\left[\left(B_{2}+A_{1}\right) I_{3 N}+B_{2} x^{*} x^{* \dagger}+x^{*} a_{1}^{\dagger}\right]
\end{array}\right) .
\end{gathered}
$$

Multiplying (4.1.10) by $\frac{2}{1+\bar{R}^{2}}=1-\frac{1}{t} A_{1}-\frac{1}{2 t^{2}}\left[2 A_{2}+a_{1}^{\dagger} a_{1}+1-2 A_{1}^{2}\right]$ has the effect of adding to the right hand side of (4.1.10):

$$
\left(\begin{array}{cc}
-\frac{1}{t^{2}} A_{1} I_{3 N} & -\frac{1}{t} A_{1} x^{*} x^{* \dagger}-\frac{1}{t^{2}} A_{1}\left[x^{*} a_{1}^{\dagger}+a_{1} x^{* \dagger}\right] \\
-\frac{1}{2 t^{2}}\left[2 A_{2}+a_{1}^{\dagger} a_{1}+1-2 A_{1}^{2}\right] x^{*} x^{* \dagger} \\
O_{3 N}
\end{array}\right)
$$

These two add up to three of the blocks of the claimed $J_{2}$. The first term in the second line of (4.1.7) is linear in $\Delta$ and contributes

$$
-6\left(1-\bar{R}^{2}\right)^{2} \bar{G} \bar{x}^{\dagger}=-\frac{24}{t^{2}} A_{1}^{2} G^{*} x^{* \dagger}
$$

to the lower left corner of the matrix $J_{2}$, which completes the calculation, including all terms of order $1 / t^{2}$

It is reasonable to expect that the lowest order terms in $1 / t$ in each block will be more significant than the higher order terms. As part of our search for solutions $\Delta$, we will study two lower-order approximations to $J_{2}$, namely

$$
J_{0}=\left(\begin{array}{cc}
-\frac{1}{t} I_{3 N} & I_{3 N}-x^{*} x^{* \dagger} \\
-\frac{24 A_{1}^{2}}{t^{2}} G^{*} x^{* \dagger} & -\frac{1}{t}\left[I_{3 N}+x^{*} x^{* \dagger}\right]
\end{array}\right)
$$




$$
J_{1}=\left(\begin{array}{cc}
-\frac{1}{t} I_{3 N} & I_{3 N}-x^{*} x^{* \dagger} \\
-\frac{24 A_{1}^{2}}{t^{2}} G^{*} x^{* \dagger} & -\frac{1}{t}\left[x^{*} a_{1}^{\dagger}+a_{1} x^{* \dagger}-A_{1} x^{*} x^{* \dagger}\right] \\
t & -\frac{1}{t}\left[I_{3 N}+x^{*} x^{* \dagger}\right]
\end{array}\right)
$$

$J_{0}$ is the approximation to $J_{2}$ including only the lowest order terms in each block- $O(1)$ in the upper right block, $O(1 / t)$ in the diagonal blocks, and $O\left(1 / t^{2}\right)$ in the lower left block. $J_{1}$ is the approximation including all first order terms and the lowest order terms in the lower left block. We note from the framework of Elias and Gingold [34] that off-diagonal elements need not be absolutely integrable, which the upper right hand block certainly isn't. It is a priori reasonable to consider ignoring the lower left block since it is of higher order than the other blocks. The resulting system (to be called $J_{-}$) will be considered in the next Section.

To compute the non-homogenous term from (4.1.7), we express $\bar{G}_{i}$ and $\bar{L}$ through lowest order in $1 / t$ :

$$
\begin{aligned}
\bar{x}_{k}-\bar{x}_{i} & =x_{k}^{*}-x_{i}^{*}+\frac{1}{t}\left(a_{1 k}-a_{1 i}\right)+O\left(1 / t^{2}\right) \\
\bar{G}_{i} & =G_{i}^{*}+\frac{1}{t} G_{1 i}^{*}+O\left(1 / t^{2}\right) \\
\bar{L} & =\left(\frac{1}{t}+\frac{B_{2}}{t^{2}}\right) x^{* \dagger}\left(G^{*}+\frac{1}{t} G_{1}^{*}\right)=\frac{1}{t} x^{* \dagger} G^{*}+O\left(1 / t^{2}\right),
\end{aligned}
$$

so we have

$$
\begin{aligned}
\left(1-\bar{R}^{2}\right)^{3} \bar{G} & =-\frac{8 A_{1}^{3}}{t^{3}} G^{*}+O\left(1 / t^{4}\right) \\
\frac{2\left(1-\bar{R}^{2}\right)^{3}}{1+\bar{R}^{2}} \bar{L} \bar{x} & =-8 A_{1}^{3} \frac{1}{t^{4}} x^{* \dagger} G^{*} x^{*}+O\left(1 / t^{5}\right) .
\end{aligned}
$$

Now we have the linearized system for $\Delta$, with each block to lowest order in $1 / t$. We call (4.1.13) the $J_{0}$ problem

$$
\dot{\Delta}=J_{0} \Delta+F=\left(\begin{array}{cc}
-\frac{1}{t} I_{3 N} & I_{3 N}-x^{*} x^{* \dagger} \\
-\frac{24 A_{1}^{2}}{t^{2}} G^{*} x^{* \dagger} & -\frac{1}{t}\left[I_{3 N}+x^{*} x^{* \dagger}\right]
\end{array}\right) \Delta-8 A_{1}^{3}\left(\begin{array}{c}
\frac{1}{t^{4}} x^{*} x^{* \dagger} G^{*} \\
\frac{1}{t^{3}} G^{*}
\end{array}\right) .
$$

The $J_{1}$ problem is defined similarly, with the same non-homogenous terms

$$
\dot{\Delta}=J_{1} \Delta+F=\left(\begin{array}{cc}
-\frac{1}{t} I_{3 N} & I_{3 N}-x^{*} x^{* \dagger} \\
-\frac{24 A_{1}^{2}}{t^{2}} G^{*} x^{* \dagger} & -\frac{1}{t}\left[x^{*} a_{1}^{\dagger}+a_{1} x^{* \dagger}-A_{1} x^{*} x^{* \dagger}\right] \\
\frac{1}{t}\left[I_{3 N}+x^{*} x^{* \dagger}\right]
\end{array}\right) \Delta-8 A_{1}^{3}\left(\begin{array}{c}
\frac{1}{t^{4}} x^{*} x^{* \dagger} G^{*} \\
\frac{1}{t^{3}} G^{*}
\end{array}\right) .
$$

Just as the approximating system becomes less relevant if the lower left block of $J_{0}$ or $J_{1}$ is removed, we cannot ignore the non-homogenous terms, even though they are $O\left(1 / t^{3}\right)$.

We next consider the nature of the singular point at $t=\infty$. The substitution $t=1 / \tau,\urcorner(\tau)=\Delta(1 / t)$ transforms the singularity from $t=\infty$ to the origin $\tau=0$ and takes the homogeneous part of (4.1.13) to

$$
\left.\frac{d\urcorner}{d \tau}=\left(\begin{array}{cc}
\frac{1}{\tau} I_{3 N} & -\frac{1}{\tau^{2}}\left(I_{3 N}-x^{*} x^{* \dagger}\right) \\
24 A_{1}^{2} G^{*} x^{* \dagger} & \frac{1}{\tau}\left(I_{3 N}+x^{*} x^{* \dagger}\right)
\end{array}\right)\right\urcorner .
$$

This matrix has a pole of second order at $\tau=0$, so by classification of singular points given in Wasow [114], $t=\infty$ is an irregular singular point of (4.1.13), and hence of the full problem (4.1.1). ${ }^{1}$ Note the same obtains for the $J_{1}$ and $J_{2}$ problems; they also have an irregular singular point at $t=\infty$.

\footnotetext{
${ }^{1}$ Note that the under the alternate singularity classification of Coddington and Levinson [20], our singularity is referred to as "of the second kind."
} 


\subsection{The Linear System for $\Delta$}

In this Section, we seek solutions to the linear problem that decay to 0. After a very helpful coordinate transformation, we solve the $J_{0}$ problem exactly twice, solve the $J_{-}$problem, and solve the $J_{1}$ problem by finding a convergent series solution tending to 0 . This work with the linear system inspires our search for solutions to the full problem in the next Chapter.

If $y \in \mathbb{R}^{3 N}$, then there exist a scalar $a$ and a vector $b \in \mathbb{R}^{3 N}, b \perp x^{*}$ (specifically $a=y^{\dagger} x^{*}, b=y-a x^{*}$ ), such that $y=a x^{*}+b$ is the decomposition of $y$ into components parallel and perpendicular to $x^{*}$. This makes it easy to compute the image of $y$ under the operator $x^{*} x^{* \dagger}$; namely $x^{*} x^{* \dagger} y=a x^{*}$. Moreover, $G^{*} x^{* \dagger} y=a G^{*}$, and letting $y=G^{*}$ in the preceding sentence we see that $x^{*} x^{* \dagger} G^{*}$ is a vector of length $x^{* \dagger} G^{*}$ in the direction $x^{*}$; in other words, the projection of $G^{*}$ onto $x^{*}$. These observations suggest changing the basis for $\mathbb{R}^{3 N}$ so that the unit vector $x^{*}$ is one of the basis vectors. Note that $x^{* \dagger} x^{*}=1$, that the matrix $x^{*} x^{* \dagger}$ is of rank one, and consequently that $x^{*} x^{* \dagger}$ is an orthogonal projection matrix

$$
\left(x^{*} x^{* \dagger}\right)^{2}=x^{*} x^{* \dagger} x^{*} x^{* \dagger}=x^{*} x^{* \dagger}
$$

onto the line through $x^{*}$. These facts imply by linear algebra [53] that there exists a $3 N$ by $3 N$ orthogonal matrix $Q_{3 N}$ such that

$$
P:=Q_{3 N}^{\dagger}\left(x^{*} x^{* \dagger}\right) Q_{3 N}=\left[\begin{array}{cccc}
1 & 0 & \cdots & 0 \\
0 & 0 & \cdots & 0 \\
\vdots & \vdots & \ddots & \vdots \\
0 & 0 & \cdots & 0
\end{array}\right]
$$

The above is tantamount to choosing a new orthonormal basis $\left\{v_{1}=x^{*}, v_{2}, \ldots v_{3 N}\right\}$ for $\mathbb{R}^{3 N}$, whose vectors form the columns of $Q_{3 N}$, and where $v_{1}=x^{*}$ is the first element in this new basis. As in Section 3.3 the transformation

$$
\bar{x}=Q \overline{\bar{x}}, \Delta=Q \bar{\Delta}, \text { with } Q=\left(\begin{array}{cc}
Q_{3 N} & O_{3 N} \\
O_{3 N} & Q_{3 N}
\end{array}\right)
$$

simplifies the "velocity" components of the approximation considerably.

$$
\begin{aligned}
& \overline{\bar{x}}_{N+1}=1 / t+B_{2} / t^{2}+B_{3} / t^{3}+\ldots \\
& \overline{\bar{x}}_{N+j}=0,2 \leq j \leq 3 N
\end{aligned}
$$

since each "velocity" $\bar{x}_{i}$ is proportional to $x_{i}^{*}$, and $x^{*}$ takes the form $Q_{3 N}^{\dagger} x^{*}=e_{1}:=(1,0, \ldots, 0)^{\dagger}$ in the rotated coordinate system.

4.2.1. Solving the $J_{0}$ Problem. The transformation (4.2.1) takes the system (4.1.13) into

$$
\begin{gathered}
\dot{\bar{\Delta}}=Q^{\dagger} J_{0} Q \bar{\Delta}+Q^{\dagger} F=\left(\begin{array}{ll}
Q_{3 N}^{\dagger} & O_{3 N} \\
O_{3 N} & Q_{3 N}^{\dagger}
\end{array}\right)\left(\begin{array}{cc}
-\frac{1}{t} I_{3 N} & I_{3 N}-x^{*} x^{* \dagger} \\
-\frac{24 A_{1}^{2}}{t^{2}} G^{*} x^{* \dagger} & -\frac{1}{t}\left[I_{3 N}+x^{*} x^{* \dagger}\right]
\end{array}\right)\left(\begin{array}{ll}
Q_{3 N} & O_{3 N} \\
O_{3 N} & Q_{3 N}
\end{array}\right) \bar{\Delta} \\
-8 A_{1}^{3}\left(\begin{array}{ll}
Q_{3 N}^{\dagger} & O_{3 N} \\
O_{3 N} & Q_{3 N}^{\dagger}
\end{array}\right)\left(\begin{array}{c}
\frac{1}{t^{4}} x^{*} x^{* \dagger} G^{*} \\
\frac{1}{t^{3}} G^{*}
\end{array}\right) \\
=\left(\begin{array}{cc}
-\frac{1}{t} I_{3 N} & I_{3 N}-P \\
-\frac{24 A_{1}^{2}}{t^{2}} Q_{3 N}^{\dagger} G^{*} e_{1}^{\dagger} & -\frac{1}{t}\left[I_{3 N}+P\right]
\end{array}\right) \bar{\Delta}-8 A_{1}^{3}\left(\begin{array}{c}
\frac{1}{t^{4}} x^{* \dagger} G^{*} e_{1} \\
\frac{1}{t^{3}} Q_{3 N}^{\dagger} G^{*}
\end{array}\right)
\end{gathered}
$$




$$
\dot{\bar{\Delta}}=J_{0} \bar{\Delta}+F=\left(\begin{array}{cccccccc}
-\frac{1}{t} & 0 & \ldots & 0 & 0 & 0 & \ldots & 0 \\
0 & -\frac{1}{t} & \ldots & 0 & 0 & 1 & \ldots & 0 \\
\vdots & \vdots & \ddots & \vdots & \vdots & \vdots & \ddots & \vdots \\
0 & \ldots & 0 & -\frac{1}{t} & 0 & \ldots & 0 & 1 \\
& & & & & & & \\
\frac{-24 A_{1}^{2}}{t^{2}} g_{1} & 0 & \ldots & 0 & -\frac{2}{t} & 0 & \ldots & 0 \\
\frac{-24 A_{1}^{2}}{t^{2}} g_{2} & 0 & \ldots & 0 & 0 & -\frac{1}{t} & \ldots & 0 \\
\vdots & \vdots & \ddots & \vdots & \vdots & \vdots & \ddots & \vdots \\
\frac{-24 A_{1}^{2}}{t^{2}} g_{3 N} & 0 & \ldots & 0 & 0 & \ldots & 0 & -\frac{1}{t}
\end{array}\right) \bar{\Delta}-8 A_{1}^{3}\left(\begin{array}{c} 
\\
\frac{1}{t^{4}} g_{1} \\
0 \\
\vdots \\
0 \\
\frac{1}{t^{3}} g_{1} \\
\vdots \\
\frac{1}{t^{3}} g_{3 N}
\end{array}\right)
$$

where $g_{j}=v_{j}^{\dagger} G^{*}$ are the coordinates of $G^{*}$ in the basis $\left\{v_{j}\right\}$, since $Q_{3 N}^{\dagger} G^{*}$ is the vector composed of the inner products of the basis elements with $G^{*}$.

The matrix $J_{0}$ is sparse and is clearly exhibited as the sum of a diagonal matrix, a strictly upper triangular matrix, and a strictly lower triangular matrix $J=D+U+L$. The triangular matrices' squares are easily seen to be the $O_{6 N}$ matrix, as is their product $L U$. The elements of the matrix $U L$ are 0 , except for the upper left quadrant, which is zero except most of the first column

$$
\left(\begin{array}{cccc}
0 & 0 & \ldots & 0 \\
\frac{-24 A_{1}^{2}}{t^{2}} g_{2} & 0 & \ldots & 0 \\
\vdots & \vdots & \ddots & \vdots \\
\frac{-24 A_{1}^{2}}{t^{2}} g_{3 N} & 0 & \ldots & 0
\end{array}\right) .
$$

We show that $L+U$ is nilpotent:

$$
\begin{aligned}
& (L+U)^{2}=U L \\
& (L+U)^{3}=(L+U) U L=O_{6 N} .
\end{aligned}
$$

This exhibits the decomposition of $J_{0}$ into diagonal plus nilpotent matrices. The off-diagonal terms don't contribute to its eigenvalues, which are $\left\{-\frac{1}{t},-\frac{2}{t}\right\}$, and the system (4.2.2) resolves into $3 N$ pairs of equations:

$$
\begin{aligned}
\dot{\bar{\Delta}}_{1} & =-\frac{1}{t} \bar{\Delta}_{1}-\frac{8 A_{1}^{3}}{t^{4}} g_{1} \\
\dot{\bar{\Delta}}_{3 N+1} & =-\frac{24 A_{1}^{2}}{t^{2}} g_{1} \bar{\Delta}_{1}-\frac{2}{t} \bar{\Delta}_{3 N+1}-\frac{8 A_{1}^{3}}{t^{3}} g_{1} \\
\dot{\bar{\Delta}}_{j} & =-\frac{1}{t} \bar{\Delta}_{j}+\bar{\Delta}_{3 N+j} \\
\dot{\bar{\Delta}}_{3 N+j} & =-\frac{24 A_{1}^{2}}{t^{2}} g_{j} \bar{\Delta}_{1}-\frac{1}{t} \bar{\Delta}_{3 N+j}-\frac{8 A_{1}^{3}}{t^{3}} g_{j}, \text { for } 2 \leq j \leq 3 N .
\end{aligned}
$$

The first two equations are coupled, and $\infty$ is a regular singularity for the pair, improving our chances for finding solutions. In particular, the first equation can be solved exactly:

$$
\bar{\Delta}_{1}=\frac{t_{0}}{t} \bar{\Delta}_{1}\left(t_{0}\right)+\frac{1}{t} \int^{t} \frac{-8 A_{1}^{3} g_{1} d s}{s^{3}}=\frac{t_{0}}{t} \bar{\Delta}_{1}\left(t_{0}\right)-\frac{4 A_{1}^{3} g_{1}}{t_{0}^{2} t}+\frac{4 A_{1}^{3} g_{1}}{t^{3}} .
$$

The choice of $\bar{\Delta}_{1}\left(t_{0}\right)=\frac{4 A_{1}^{3} g_{1}}{t_{0}^{3}}$ gives

$$
\bar{\Delta}_{1}=\frac{4 A_{1}^{3} g_{1}}{t^{3}}=\frac{t_{0}^{3}}{t^{3}} \bar{\Delta}_{1}\left(t_{0}\right) .
$$

Next we insert this expression for $\bar{\Delta}_{1}$ into the equations for the "velocity" components:

$$
\begin{aligned}
& \dot{\bar{\Delta}}_{3 N+1}=-\frac{2}{t} \bar{\Delta}_{3 N+1}-\frac{24 A_{1}^{2} g_{1}}{t^{2}}\left(\frac{4 A_{1}^{3} g_{1}}{t^{3}}\right)-\frac{8 A_{1}^{3} g_{1}}{t^{3}}=-\frac{2}{t} \bar{\Delta}_{3 N+1}-\frac{8 A_{1}^{3} g_{1}}{t^{3}}-\frac{96 A_{1}^{5} g_{1}^{2}}{t^{5}} \\
& \dot{\bar{\Delta}}_{3 N+j}=-\frac{1}{t} \bar{\Delta}_{3 N+j}-\frac{24 A_{1}^{2} g_{j}}{t^{2}}\left(\frac{4 A_{1}^{3} g_{1}}{t^{3}}\right)-\frac{8 A_{1}^{3} g_{j}}{t^{3}}=-\frac{1}{t} \bar{\Delta}_{3 N+j}-\frac{8 A_{1}^{3} g_{j}}{t^{3}}-\frac{96 A_{1}^{5} g_{1} g_{j}}{t^{5}}, \text { for } 2 \leq j \leq 3 N .
\end{aligned}
$$


The solutions are

$$
\begin{aligned}
\bar{\Delta}_{3 N+1} & =\frac{t_{0}^{2}}{t^{2}} \bar{\Delta}_{3 N+1}\left(t_{0}\right)-\frac{1}{t^{2}} \int_{t_{0}}^{t}\left[\frac{8 A_{1}^{3} g_{1}}{s}+\frac{96 A_{1}^{5} g_{1}^{2}}{s^{3}}\right] d s \\
& =\frac{t_{0}^{2}}{t^{2}} \bar{\Delta}_{3 N+1}\left(t_{0}\right)-\frac{8 A_{1}^{3} g_{1} \log \frac{t}{t_{0}}}{t^{2}}+\frac{48 A_{1}^{5} g_{1}^{2}}{t^{4}}-\frac{48 A_{1}^{5} g_{1}^{2}}{t_{0}^{2} t^{2}} \\
\bar{\Delta}_{3 N+j} & =\frac{t_{0}}{t} \bar{\Delta}_{3 N+j}\left(t_{0}\right)-\frac{1}{t} \int_{t_{0}}^{t}\left[\frac{8 A_{1}^{3} g_{j}}{s^{2}}+\frac{96 A_{1}^{5} g_{1} g_{j}}{s^{4}}\right] d s \\
& =\frac{t_{0}}{t} \bar{\Delta}_{3 N+j}\left(t_{0}\right)+\frac{8 A_{1}^{3} g_{j}}{t^{2}}-\frac{8 A_{1}^{3} g_{j}}{t_{0} t}+\frac{32 A_{1}^{5} g_{1} g_{j}}{t^{4}}-\frac{32 A_{1}^{5} g_{1} g_{j}}{t_{0}^{3} t}
\end{aligned}
$$

The choices

$$
\begin{aligned}
& \bar{\Delta}_{3 N+1}\left(t_{0}\right)=\frac{48 A_{1}^{5} g_{1}^{2}}{t_{0}^{4}} \\
& \bar{\Delta}_{3 N+j}\left(t_{0}\right)=\frac{8 A_{1}^{3} g_{j}}{t_{0}^{2}}+\frac{32 A_{1}^{5} g_{1} g_{j}}{t_{0}^{4}}
\end{aligned}
$$

lead to

$$
\begin{aligned}
& \bar{\Delta}_{3 N+1}=-\frac{8 A_{1}^{3} g_{1} \log t / t_{0}}{t^{2}}+\frac{48 A_{1}^{5} g_{1}^{2}}{t^{4}} \\
& \bar{\Delta}_{3 N+j}=\frac{8 A_{1}^{3} g_{j}}{t^{2}}+\frac{32 A_{1}^{5} g_{1} g_{j}}{t^{4}} .
\end{aligned}
$$

Lastly we have

$$
\begin{aligned}
\bar{\Delta}_{j} & =\frac{t_{0}}{t} \bar{\Delta}_{j}\left(t_{0}\right)+\frac{1}{t} \int_{t_{0}}^{t}\left[\frac{8 A_{1}^{3} g_{j}}{s}+\frac{32 A_{1}^{5} g_{1} g_{j}}{s^{3}}\right] d s \\
& =\frac{t_{0}}{t} \bar{\Delta}_{j}\left(t_{0}\right)+\frac{8 A_{1}^{3} g_{j} \log \frac{t}{t_{0}}}{t}-\frac{16 A_{1}^{5} g_{1} g_{j}}{t^{3}}+\frac{16 A_{1}^{5} g_{1} g_{j}}{t_{0}^{2} t}
\end{aligned}
$$

The choice

$$
\bar{\Delta}_{j}\left(t_{0}\right)=\frac{16 A_{1}^{5} g_{1} g_{j}}{t_{0}^{3}}
$$

gives

$$
\bar{\Delta}_{j}=\frac{8 A_{1}^{3} g_{j} \log \frac{t}{t_{0}}}{t}-\frac{16 A_{1}^{5} g_{1} g_{j}}{t^{3}} .
$$

We summarize the asymptotic behavior of this solution $\bar{\Delta}$ and $\Delta$ as $t \rightarrow \infty$ :

$$
\left(\begin{array}{c}
\bar{\Delta}_{1} \\
\bar{\Delta}_{j} \\
\bar{\Delta}_{3 N+1} \\
\bar{\Delta}_{3 N+j}
\end{array}\right)=\left(\begin{array}{c}
O\left(1 / t^{3}\right) \\
O(\log t / t) \\
O\left(\log t / t^{2}\right) \\
O\left(1 / t^{2}\right)
\end{array}\right), \quad\left(\begin{array}{c}
\Delta_{j} \\
\Delta_{N+j}
\end{array}\right)=\left(\begin{array}{c}
O(\log t / t) \\
O\left(\log t / t^{2}\right)
\end{array}\right)
$$

These go to 0 as $t \rightarrow \infty$, but slowly.

Now we try a different approach and solve the $J_{0}$ problem by finding a formal series; we suppose

$$
\bar{\Delta}_{1}=\sum_{r=0}^{\infty} \frac{c_{r}}{t^{r}} \quad \bar{\Delta}_{3 N+1}=\sum_{r=0}^{\infty} \frac{d_{r}}{t^{r}},
$$

then the first pair of (4.2.3) become

$$
\begin{aligned}
& -\sum_{r=2}^{\infty} \frac{(r-1) c_{r-1}}{t^{r}}=-\sum_{r=1}^{\infty} \frac{c_{r-1}}{t^{r}}-\frac{8 A_{1}^{3} g_{1}}{t^{4}} \\
& -\sum_{r=2}^{\infty} \frac{(r-1) d_{r-1}}{t^{r}}=-24 A_{1}^{2} g_{1} \sum_{r=2}^{\infty} \frac{c_{r-2}}{t^{r}}-2 \sum_{r=1}^{\infty} \frac{d_{r-1}}{t^{r}}-\frac{8 A_{1}^{3} g_{1}}{t^{3}} .
\end{aligned}
$$


Equating coefficients of like powers yields algebraic equations. For $r=1$, we have $c_{0}=d_{0}=0$. For $r=2$,

$$
\begin{aligned}
c_{1} & =c_{1} \\
d_{1} & =24 A_{1}^{2} g_{1} c_{0}+2 d_{1}=2 d_{1},
\end{aligned}
$$

so $d_{1}=0$, but $c_{1}$ is not yet determined. For $r=3$,

$$
\begin{aligned}
& 2 c_{2}=c_{2} \\
& 2 d_{2}=24 A_{1}^{2} g_{1} c_{1}+2 d_{2}+8 A_{1}^{3} g_{1},
\end{aligned}
$$

so $c_{2}=0$ and $c_{1}=-A_{1} / 3$, but $d_{2}$ is not yet determined. For $r=4$,

$$
\begin{aligned}
3 c_{3} & =c_{3}+8 A_{1}^{3} g_{1} \\
3 d_{3} & =24 A_{1}^{2} g_{1} c_{2}+2 d_{3}=2 d_{3},
\end{aligned}
$$

which imply $c_{3}=4 A_{1}^{3} g_{1}$ and $d_{3}=0$. For $r=5$,

$$
\begin{aligned}
4 c_{4} & =c_{4} \\
4 d_{4} & =24 A_{1}^{2} g_{1} c_{3}+2 d_{4},
\end{aligned}
$$

so $c_{4}=0$ and $d_{4}=12 A_{1}^{2} g_{1} c_{3}=48 A_{1}^{5} g_{1}^{2}$. So far we have the free parameter, $d_{2}$. There is a recurrence relation (for $r>5$ ):

$$
\begin{aligned}
& (r-2) c_{r-1}=0 \\
& (r-3) d_{r-1}=24 A_{1}^{2} g_{1} c_{r-2}
\end{aligned}
$$

so $c_{r}=d_{r}=0$ for $r>4$, and we have

$$
\begin{aligned}
\bar{\Delta}_{1} & =-\frac{A_{1}}{3 t}+\frac{4 A_{1}^{3} g_{1}}{t^{3}} \\
\bar{\Delta}_{3 N+1} & =\frac{d_{2}}{t^{2}}+\frac{48 A_{1}^{5} g_{1}^{2}}{t^{4}} .
\end{aligned}
$$

The equations for $\bar{\Delta}_{j}$ and $\bar{\Delta}_{3 N+j}$ can now be solved in terms of the $c_{r}$ :

$$
\begin{aligned}
\dot{\bar{\Delta}}_{3 N+j} & =-\frac{24 A_{1}^{2} g_{j}}{t^{2}}\left(-\frac{A_{1}}{3 t}+\frac{4 A_{1}^{3} g_{1}}{t^{3}}\right)-\frac{1}{t} \bar{\Delta}_{3 N+j}-\frac{8 A_{1}^{3} g_{j}}{t^{3}}=-\frac{1}{t} \bar{\Delta}_{3 N+j}-\frac{96 A_{1}^{5} g_{1} g_{j}}{t^{5}} \\
\bar{\Delta}_{3 N+j} & =\frac{c}{t}-\frac{1}{t} \int^{t} \frac{96 A_{1}^{5} g_{1} g_{j}}{s^{4}} d s=\frac{32 A_{1}^{5} g_{1} g_{j}}{t^{4}}
\end{aligned}
$$

where we have chosen $c=\frac{32 A_{1}^{5} g_{1} g_{j}}{t_{0}^{4}}$ to cancel the integral evaluation at the lower limit. Then

$$
\begin{aligned}
& \dot{\bar{\Delta}}_{j}=-\frac{1}{t} \bar{\Delta}_{j}+\frac{32 A_{1}^{5} g_{1} g_{j}}{t^{4}} \\
& \bar{\Delta}_{j}=\frac{d}{t}+\frac{1}{t} \int^{t} \frac{32 A_{1}^{5} g_{1} g_{j}}{s^{3}}=-\frac{16 A_{1}^{5} g_{1} g_{j}}{t^{3}}
\end{aligned}
$$

where we have chosen $d=-\frac{16 A_{1}^{6} g_{1} g_{j}}{t_{0}^{3}}$ to cancel the evaluation of the integral at the lower limit. This solution can be rotated back to $\Delta$, and we have

$$
\left(\begin{array}{c}
\Delta_{j} \\
\Delta_{3 N+j}
\end{array}\right)=\left(\begin{array}{c}
O(1 / t) \\
O\left(1 / t^{2}\right)
\end{array}\right)
$$

This convergence to 0 is faster than that of the solution exhibited on the previous page.

We pause to consider the simpler $J_{-}$problem, which seeks the solution to 


$$
\dot{\bar{\Delta}}=J_{-} \bar{\Delta}+F=\left(\begin{array}{cccccccc}
-\frac{1}{t} & 0 & \ldots & 0 & 0 & 0 & \ldots & 0 \\
0 & -\frac{1}{t} & \ldots & 0 & 0 & 1 & \ldots & 0 \\
\vdots & \vdots & \ddots & \vdots & \vdots & \vdots & \ddots & \vdots \\
0 & \ldots & 0 & -\frac{1}{t} & 0 & \ldots & 0 & 1 \\
0 & 0 & \ldots & 0 & -\frac{2}{t} & 0 & \ldots & 0 \\
0 & 0 & \ldots & 0 & 0 & -\frac{1}{t} & \ldots & 0 \\
\vdots & \vdots & \ddots & \vdots & \vdots & \vdots & \ddots & \vdots \\
0 & 0 & \ldots & 0 & 0 & \ldots & 0 & -\frac{1}{t}
\end{array}\right) \bar{\Delta}-8 A_{1}^{3}\left(\begin{array}{c} 
\\
\frac{1}{t^{4}} g_{1} \\
0 \\
\vdots \\
0 \\
\frac{1}{t^{3}} g_{1} \\
\vdots \\
\frac{1}{t^{3}} g_{3 N}
\end{array}\right),
$$

where we have already taken advantage of the coordinate transformation given at the beginning of this Section. We seek a brute-force solution for comparison with the $J_{0}$ results. As above, the system resolves into $3 N$ pairs of equations:

$$
\begin{aligned}
\dot{\bar{\Delta}}_{1} & =-\frac{1}{t} \bar{\Delta}_{1}-\frac{8 A_{1}^{3}}{t^{4}} g_{1} \\
\dot{\bar{\Delta}}_{3 N+1} & =-\frac{2}{t} \bar{\Delta}_{3 N+1}-\frac{8 A_{1}^{3}}{t^{3}} g_{1} \\
\dot{\bar{\Delta}}_{j} & =-\frac{1}{t} \bar{\Delta}_{j}+\bar{\Delta}_{3 N+j} \\
\dot{\bar{\Delta}}_{3 N+j} & =-\frac{1}{t} \bar{\Delta}_{3 N+j}-\frac{8 A_{1}^{3}}{t^{3}} g_{j}, \text { for } 2 \leq j \leq 3 N .
\end{aligned}
$$

Again, the first two equations have a regular singularity at $t=\infty$. All but the $\bar{\Delta}_{j}, 1<j \leq 3 N$ equation can be solved immediately:

$$
\begin{aligned}
\bar{\Delta}_{1} & =\frac{4 A_{1}^{3} g_{1}}{t^{3}} \\
\bar{\Delta}_{3 N+1} & =\frac{t_{0}^{2}}{t^{2}} \bar{\Delta}_{3 N+1}\left(t_{0}\right)-\frac{8 A_{1}^{3} g_{1} \log \frac{t}{t_{0}}}{t^{2}} \\
\bar{\Delta}_{3 N+j} & =\frac{8 A_{1}^{3} g_{j}}{t^{2}} .
\end{aligned}
$$

Then the $\bar{\Delta}_{j}, 1<j \leq 3 N$ equation becomes

$$
\begin{aligned}
& \dot{\bar{\Delta}}_{j}=-\frac{1}{t} \bar{\Delta}_{j}+\frac{8 A_{1}^{3} g_{j}}{t^{2}} \\
& \bar{\Delta}_{j}=-\frac{8 A_{1}^{3} g_{j} \log \frac{t}{t_{0}}}{t}
\end{aligned}
$$

with the asymptotic behavior

$$
\left(\begin{array}{c}
\Delta_{j} \\
\Delta_{N+j}
\end{array}\right)=\left(\begin{array}{c}
O(\log t / t) \\
O\left(\log t / t^{2}\right)
\end{array}\right)
$$

4.2.2. The $J_{1}$ Problem. The next step is to augment the coefficient matrix, adding terms of order $1 / t$ to the upper right block. We replace (4.1.13) with

$$
\dot{\Delta}=J_{1} \Delta+F=\left(\begin{array}{cc}
-\frac{1}{t} I & -\frac{1}{t}\left[x^{*} a_{1}^{\dagger}+a_{1} x^{* \dagger}-A_{1} x^{*} x^{* \dagger}\right] \\
-\frac{24 A_{1}^{2}}{t^{2}} G^{*} x^{* \dagger} & -\frac{1}{t}\left(I+x^{*} x^{* \dagger}\right)
\end{array}\right) \Delta-8 A_{1}^{3}\left(\begin{array}{c}
\frac{1}{t^{4}} x^{*} x^{* \dagger} G^{*} \\
\frac{1}{t^{3}} G^{*}
\end{array}\right) .
$$


Since the vector $a_{1}$ is within the linear span of $v_{1}=x^{*}=\bar{x}_{N+j}\left(t_{0}\right) / \sqrt{A\left(t_{0}\right)}$ and $\bar{x}_{j}\left(t_{0}\right)$, it makes sense to choose $v_{2}$, the second basis element of $\mathbb{R}^{3 N}$ and the second column of the matrix $Q_{3 N}$, via the GrahamSchmidt process starting with $v_{1}=x^{*}$ and $\bar{x}_{j}\left(t_{0}\right)$. Then we have

$$
\begin{aligned}
v_{2 j} & =\frac{\sqrt{A\left(t_{0}\right)} \bar{x}_{j}\left(t_{0}\right)-h\left(t_{0}\right) x_{j}^{*}}{\sqrt{A\left(t_{0}\right)\left(\bar{R}\left(t_{0}\right)^{2}-A\left(t_{0}\right)\right)-h\left(t_{0}\right)^{2}}}, \text { for } 1 \leq j \leq 3 N \\
\bar{x}_{j}\left(t_{0}\right) & =\frac{h\left(t_{0}\right)}{\sqrt{A\left(t_{0}\right)}} x_{j}^{*}+\sqrt{\bar{R}\left(t_{0}\right)^{2}-A\left(t_{0}\right)-\frac{h\left(t_{0}\right)^{2}}{A\left(t_{0}\right)}} v_{2 j} .
\end{aligned}
$$

Note that The quantity $\bar{R}\left(t_{0}\right)^{2}-A\left(t_{0}\right)-\frac{h\left(t_{0}\right)^{2}}{A\left(t_{0}\right)} \geq 0$ by the Schwartz inequality, and is 0 only in the case that the initial position $3 N$-vector is parallel to the initial velocity; $\bar{x}_{i}\left(t_{0}\right)=\gamma \bar{x}_{N+i}\left(t_{0}\right), i=1, \ldots 3 N$, for some real $\gamma$. Assuming it is positive, we can rewrite (3.3.19) as

$$
a_{1}=A_{1} x^{*}+b v_{2}, \text { where } b=\sqrt{\bar{R}\left(t_{0}\right)^{2}-A\left(t_{0}\right)-\frac{h\left(t_{0}\right)^{2}}{A\left(t_{0}\right)}}
$$

In the $\left\{v_{j}\right\}$ basis, we have near the boundary

$$
\overline{\bar{x}}=\left(\begin{array}{c}
1+A_{1} / t+A_{2} / t^{2}+\ldots \\
b / t+b B_{2} / t^{2}+\ldots \\
0 \\
\vdots \\
0 \\
1 / t+B_{2} / t^{2}+\ldots \\
0 \\
\vdots \\
0
\end{array}\right)
$$

and

$$
x^{*} a_{1}^{\dagger}=\left(\begin{array}{ccccc}
A_{1} & b & 0 & \ldots & 0 \\
0 & 0 & 0 & \ldots & 0 \\
\vdots & \vdots & \vdots & \ddots & \vdots \\
0 & 0 & 0 & \ldots & 0
\end{array}\right)
$$

so the additional term in the upper right block is

$$
-\frac{1}{t}\left(\begin{array}{ccccc}
A_{1} & b & 0 & \ldots & 0 \\
b & 0 & 0 & \ldots & 0 \\
0 & \vdots & \ddots & & \vdots \\
\vdots & \vdots & \vdots & \ddots & \vdots \\
0 & 0 & 0 & \ldots & 0
\end{array}\right)
$$


and the $J 1$ problem takes the form $(4.2 .9)$

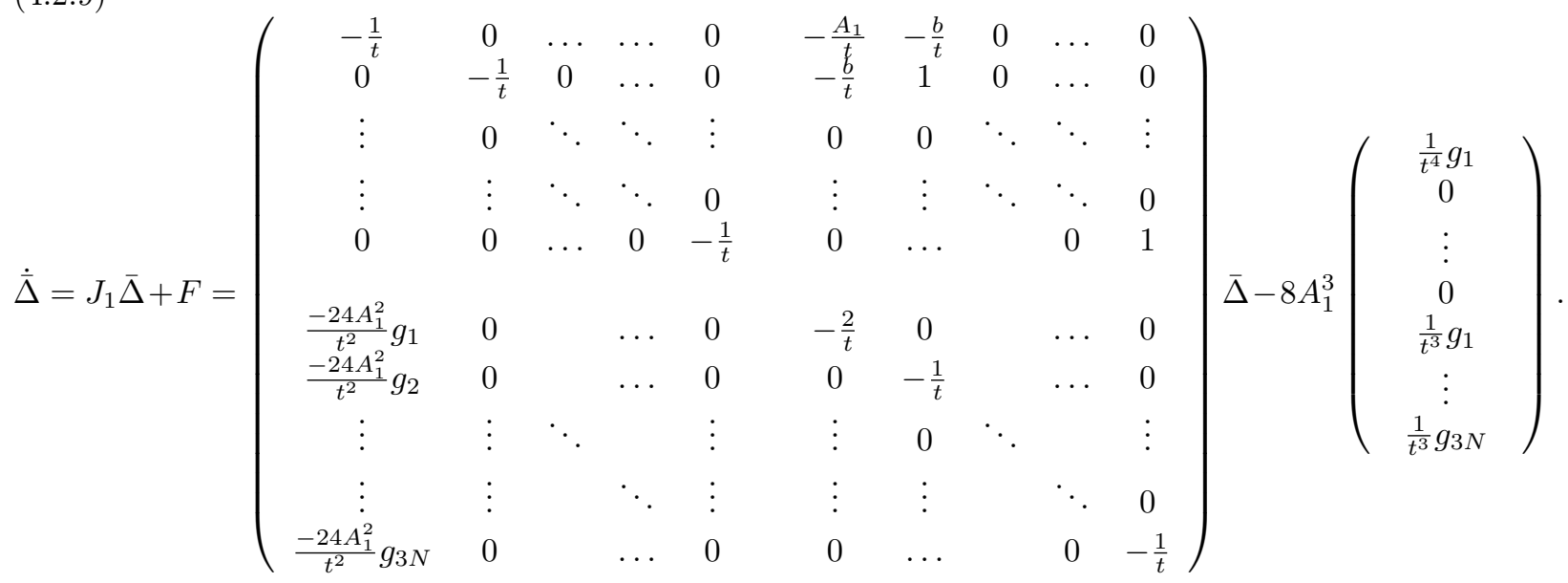

We have introduced a coupling among $\bar{\Delta}_{1}, \bar{\Delta}_{2}, \bar{\Delta}_{3 N+1}$, and $\bar{\Delta}_{3 N+2}$, but doesn't change the other components of $(4.2 .3)$

$$
\begin{aligned}
\dot{\bar{\Delta}}_{1} & =-\frac{1}{t} \bar{\Delta}_{1}-\frac{A_{1}}{t} \bar{\Delta}_{3 N+1}-\frac{b}{t} \bar{\Delta}_{3 N+2}-\frac{8 A_{1}^{3} g_{1}}{t^{4}} \\
\dot{\bar{\Delta}}_{2} & =-\frac{1}{t} \bar{\Delta}_{2}-\frac{b}{t} \bar{\Delta}_{3 N+1}+\bar{\Delta}_{3 N+2} \\
\dot{\bar{\Delta}}_{3 N+1} & =-\frac{24 A_{1}^{2} g_{1}}{t^{2}} \bar{\Delta}_{1}-\frac{2}{t} \bar{\Delta}_{3 N+1}-\frac{8 A_{1}^{3} g_{1}}{t^{3}} \\
\dot{\bar{\Delta}}_{3 N+2} & =-\frac{24 A_{1}^{2} g_{2}}{t^{2}} \bar{\Delta}_{1}-\frac{1}{t} \bar{\Delta}_{3 N+2}-\frac{8 A_{1}^{3} g_{2}}{t^{3}} .
\end{aligned}
$$

The other three don't depend on $\bar{\Delta}_{2}$, so consider the system

$$
\left(\begin{array}{c}
\dot{\bar{\Delta}}_{1} \\
\dot{\bar{\Delta}}_{3 N+1} \\
\dot{\bar{\Delta}}_{3 N+2}
\end{array}\right)=\left(\begin{array}{ccc}
-\frac{1}{t} & -\frac{A_{1}}{t} & -\frac{b}{t} \\
-\frac{24 A_{1}^{2} g_{1}}{t^{2}} & -\frac{2}{t} & 0 \\
-\frac{24 A_{1}^{2} g_{2}}{t^{2}} & 0 & -\frac{1}{t}
\end{array}\right)\left(\begin{array}{c}
\bar{\Delta}_{1} \\
\bar{\Delta}_{3 N+1} \\
\bar{\Delta}_{3 N+2}
\end{array}\right)-8 A_{1}^{3}\left(\begin{array}{c}
\frac{g_{1}}{t^{4}} \\
\frac{g_{1}}{t^{3}} \\
\frac{g_{2}}{t^{3}}
\end{array}\right),
$$

which is easily seen to have a regular singular point at $t=\infty$. The eigenvalues of the matrix are the zeros of the cubic

$$
-\frac{24 A_{1}^{2} g_{2}}{t^{2}}\left(\lambda+\frac{2}{t}\right) \frac{b}{t}+\left(\lambda+\frac{1}{t}\right)\left[\left(\lambda+\frac{1}{t}\right)\left(\lambda+\frac{2}{t}\right)-\frac{24 A_{1}^{3} g_{1}}{t^{3}}\right] .
$$

Using these substitutions

$$
\begin{aligned}
& a_{1}=24 A_{1}^{2} g_{1} \\
& a_{2}=24 A_{1}^{2} g_{2}
\end{aligned}
$$

so the formulas fit on the page, Maxima gives the first few terms in $1 / t$ of the eigenvalues:

$$
\begin{aligned}
& \lambda_{1}=-\frac{1}{t}+\sqrt{a_{2} b} \frac{1}{t^{3 / 2}}+\frac{a_{1} A_{1}}{2 t^{2}}+\frac{a_{1}^{2} A_{1}^{2}-4 a_{1} a_{2} A_{1} b}{8 \sqrt{a_{2} b} t^{5 / 2}}-\frac{a_{1}^{2} A_{1}^{2}-a_{1} a_{2} A_{1} b}{2 t^{3}}+o\left(\frac{1}{t^{3}}\right) \\
& \lambda_{2}=-\frac{1}{t}-\sqrt{a_{2} b} \frac{1}{t^{3 / 2}}+\frac{a_{1} A_{1}}{2 t^{2}}-\frac{a_{1}^{2} A_{1}^{2}-4 a_{1} a_{2} A_{1} b}{8 \sqrt{a_{2} b} t^{5 / 2}}-\frac{a_{1}^{2} A_{1}^{2}-a_{1} a_{2} A_{1} b}{2 t^{3}}+o\left(\frac{1}{t^{3}}\right) \\
& \lambda_{3}=-\frac{2}{t}-\frac{a_{1} A_{1}}{t^{2}}+\frac{a_{1}^{2} A_{1}^{2}-a_{1} a_{2} A_{1} b}{t^{3}}+o\left(\frac{1}{t^{3}}\right) .
\end{aligned}
$$

The eigenvalues are real $\left(+o\left(1 / t^{3}\right)\right)$ provided $g_{2} \geq 0$. Finding no way to proceed toward a solution, we next seek a series solution in $1 / t$. 
Assuming $b \neq 0$, which holds unless the $3 N$-position vector $\left(\bar{x}_{1}\left(t_{0}\right), \ldots, \bar{x}_{N}\left(t_{0}\right)\right)^{\dagger}$ is parallel to the $3 N$ velocity vector $\left(\bar{x}_{N+1}\left(t_{0}\right), \ldots, \bar{x}_{2 N}\left(t_{0}\right)\right)^{\dagger}$, we will show the $J_{1}$ problem admits a convergent power series solution. We start by showing the system of three equations (4.2.11) has one, and it will then be straightforward to find convergent power series for the rest of the equations, and thus for the whole $J_{1}$ problem. We suppose

$$
\bar{\Delta}_{1}=\sum_{r=0}^{\infty} \frac{c_{r}}{t^{r}} \quad \bar{\Delta}_{N+1}=\sum_{r=0}^{\infty} \frac{d_{r}}{t^{r}} \quad \bar{\Delta}_{N+2}=\sum_{r=0}^{\infty} \frac{e_{r}}{t^{r}}
$$

then the equations become

$$
\begin{aligned}
& \sum_{r=2}^{\infty} \frac{(r-1) c_{r-1}}{t^{r}}=\sum_{r=1}^{\infty} \frac{c_{r-1}}{t^{r}}+A_{1} \sum_{r=1}^{\infty} \frac{d_{r-1}}{t^{r}}+b \sum_{r=1}^{\infty} \frac{e_{r-1}}{t^{r}}+\frac{8 A_{1}^{3} g_{1}}{t^{4}} \\
& \sum_{r=2}^{\infty} \frac{(r-1) d_{r-1}}{t^{r}}=24 A_{1}^{2} g_{1} \sum_{r=2}^{\infty} \frac{c_{r-2}}{t^{r}}+2 \sum_{r=1}^{\infty} \frac{d_{r-1}}{t^{r}}+\frac{8 A_{1}^{3} g_{1}}{t^{3}} \\
& \sum_{r=2}^{\infty} \frac{(r-1) e_{r-1}}{t^{r}}=24 A_{1}^{2} g_{2} \sum_{r=2}^{\infty} \frac{c_{r-2}}{t^{r}}+\sum_{r=1}^{\infty} \frac{e_{r-1}}{t^{r}}+\frac{8 A_{1}^{3} g_{2}}{t^{3}}
\end{aligned}
$$

Again, equating coefficients of like powers yields algebraic equations. For $r=1$, we have

$$
\begin{aligned}
& 0=c_{0}+A_{1} d_{0}+b e_{0} \\
& 0=2 d_{0} \\
& 0=e_{0},
\end{aligned}
$$

so $c_{0}=d_{0}=e_{0}=0$. For $r=2$,

$$
\begin{aligned}
c_{1} & =c_{1}+A_{1} d_{1}+b e_{1} \\
d_{1} & =2 d_{1} \\
e_{1} & =e_{1},
\end{aligned}
$$

so $d_{1}=0$ by the second equation, then $e_{1}=0$ by the first, but $c_{1}$ is not yet determined. For $r=3$,

$$
\begin{aligned}
& 2 c_{2}=c_{2}+A_{1} d_{2}+b e_{2} \\
& 2 d_{2}=24 A_{1}^{2} g_{1} c_{1}+2 d_{2}+8 A_{1}^{3} g_{1} \\
& 2 e_{2}=24 A_{1}^{2} g_{2} c_{1}+e_{2}+8 A_{1}^{3} g_{2} .
\end{aligned}
$$

The second equation gives $c_{1}=-A_{1} / 3$, then the third gives $e_{2}=0$, so $c_{2}=A_{1} d_{2}$. For $r=4$,

$$
\begin{aligned}
3 c_{3} & =c_{3}+A_{1} d_{3}+b e_{3}+8 A_{1}^{3} g_{1} \\
3 d_{3} & =24 A_{1}^{2} g_{1} c_{2}+2 d_{3} \\
3 e_{3} & =24 A_{1}^{2} g_{2} c_{2}+e_{3} .
\end{aligned}
$$

The second and third equations simplify:

$$
\begin{aligned}
& d_{3}=24 A_{1}^{2} g_{1} c_{2}=24 A_{1}^{3} g_{1} d_{2} \\
& e_{3}=12 A_{1}^{2} g_{2} c_{2}=12 A_{1}^{3} g_{2} d_{2},
\end{aligned}
$$

and then

$$
c_{3}=\frac{1}{2}\left(A_{1} d_{3}+b e_{3}\right)+4 A_{1}^{3} g_{1}=6 A_{1}^{3}\left(2 A_{1} g_{1}+b g_{2}\right) d_{2}+4 A_{1}^{3} g_{1},
$$

expressed in terms of the free parameter $d_{2}$.

There is a recurrence relation for $r>4$ :

$$
\begin{aligned}
(r-2) c_{r-1} & =A_{1} d_{r-1}+b e_{r-1} \\
(r-3) d_{r-1} & =24 A_{1}^{2} g_{1} c_{r-2} \\
(r-2) e_{r-1} & =24 A_{1}^{2} g_{2} c_{r-2} .
\end{aligned}
$$


We shift the index, which gives for $r>3$

$$
\begin{aligned}
c_{r} & =\frac{A_{1} d_{r}+b e_{r}}{r-1}=\frac{24 A_{1}^{2}}{(r-1)}\left(\frac{A_{1} g_{1}}{r-2}+\frac{b g_{2}}{r-1}\right) c_{r-1} \\
d_{r} & =\frac{24 A_{1}^{2} g_{1} c_{r-1}}{r-2} \\
e_{r} & =\frac{24 A_{1}^{2} g_{2} c_{r-1}}{r-1} .
\end{aligned}
$$

From the recurrence relation, we can apply the ratio test for convergence of the $c_{r}$ series:

$$
\left|\frac{c_{r}}{c_{r-1}}\right|=\frac{24 A_{1}^{2}}{(r-1)}\left|\frac{A_{1} g_{1}}{r-2}+\frac{b g_{2}}{r-1}\right| \rightarrow 0,
$$

as $r \rightarrow \infty$, so the power series for $\bar{\Delta}_{1}$ converges for all $t$. The absolute value of every term in the $d_{r}$ and $e_{r}$ series is less than the absolute value of the corresponding term in the $c_{r}$ series, so they also converge for all $t$. All three series are absolutely convergent for all $t$, and each series represents a real analytic function. We summarize the results of this calculation:

$$
\begin{aligned}
\bar{\Delta}_{1} & =-\frac{A_{1}}{3 t}+\frac{A_{1} d_{2}}{t^{2}}+\frac{6 A_{1}^{3}\left(2 A_{1} g_{1}+b g_{2}\right) d_{2}+4 A_{1}^{3} g_{1}}{t^{3}}+\sum_{r=4}^{\infty} \frac{c_{r}}{t^{r}} \\
\bar{\Delta}_{3 N+1} & =\frac{d_{2}}{t^{2}}+\frac{24 A_{1}^{3} g_{1} d_{2}}{t^{3}}+24 A_{1}^{2} g_{1} \sum_{r=4}^{\infty} \frac{c_{r-1}}{(r-2) t^{r}} \\
\bar{\Delta}_{3 N+2} & =\frac{12 A_{1}^{3} g_{2} d_{2}}{t^{3}}+24 A_{1}^{2} g_{2} \sum_{r=4}^{\infty} \frac{c_{r-1}}{(r-1) t^{r}} .
\end{aligned}
$$

The equations for $\bar{\Delta}_{j}$ and $\bar{\Delta}_{3 N+j}$ can now be solved in terms of the $c_{r}$. First, for $j>2$,

$$
\begin{aligned}
\dot{\bar{\Delta}}_{3 N+j} & =-\frac{1}{t} \bar{\Delta}_{3 N+j}-\frac{24 A_{1}^{2} g_{j}}{t^{2}} \sum_{r=1}^{\infty} \frac{c_{r}}{t^{r}}-\frac{8 A_{1}^{3} g_{j}}{t^{3}}=-\frac{1}{t} \bar{\Delta}_{3 N+j}-24 A_{1}^{2} g_{j} \sum_{r=4}^{\infty} \frac{c_{r-2}}{t^{r}} \\
\bar{\Delta}_{3 N+j} & =\frac{d}{t}-\frac{1}{t} \int^{t} 24 A_{1}^{2} g_{j} \sum_{r=4}^{\infty} \frac{c_{r-2}}{s^{r-1}} d s=24 A_{1}^{2} g_{j} \sum_{r=4}^{\infty} \frac{c_{r-2}}{(r-2) t^{r-1}} \\
& =24 A_{1}^{2} g_{j} \sum_{r=3}^{\infty} \frac{c_{r-1}}{(r-1) t^{r}}=24 A_{1}^{2} g_{j} \frac{A_{1} d_{2}}{2 t^{3}}+\sum_{r=4}^{\infty} \frac{c_{r-1}}{(r-1) t^{r}},
\end{aligned}
$$

where we have chosen $d$ to cancel the integral term at the lower limit. The absolute value of every term in this series is less than the absolute value of $\left|c_{r-1}\right|$ so the series converges for all $t$ since the $c_{r}$ series does. Then

$$
\begin{aligned}
\dot{\bar{\Delta}}_{2} & =-\frac{1}{t} \bar{\Delta}_{2}-\frac{b d_{2}}{t^{3}}-24 b A_{1}^{2} g_{1} \sum_{r=4}^{\infty} \frac{c_{r-2}}{(r-3) t^{r}}+24 A_{1}^{2} g_{2} \sum_{r=3}^{\infty} \frac{c_{r-1}}{(r-2) t^{r}} \\
\bar{\Delta}_{2} & =\frac{e}{t}+\frac{1}{t} \int^{t}\left[-\frac{b d_{2}}{s^{2}}-24 b A_{1}^{2} g_{1} \sum_{r=4}^{\infty} \frac{c_{r-2}}{(r-3) s^{r-1}}+24 A_{1}^{2} g_{2} \sum_{r=3}^{\infty} \frac{c_{r-1}}{(r-2) s^{r-1}}\right] d s \\
& =\frac{b d_{2}}{t^{2}}+24 b A_{1}^{2} g_{1} \sum_{r=4}^{\infty} \frac{c_{r-2}}{(r-3)(r-2) t^{r-1}}-24 A_{1}^{2} g_{2} \sum_{r=3}^{\infty} \frac{c_{r-1}}{(r-2)^{2} t^{r-1}} \\
& =\frac{b-24 A_{1}^{3} g_{2}}{t^{2}} d_{2}+24 b A_{1}^{2} g_{1} \sum_{r=3}^{\infty} \frac{c_{r-1}}{(r-1)(r-2) t^{r}}-24 A_{1}^{2} g_{2} \sum_{r=3}^{\infty} \frac{c_{r}}{(r-1)^{2} t^{r}},
\end{aligned}
$$


and for $j>2$,

$$
\begin{aligned}
\dot{\bar{\Delta}}_{j} & =-\frac{1}{t} \bar{\Delta}_{j}+24 A_{1}^{2} g_{j} \sum_{r=3}^{\infty} \frac{c_{r-1}}{(r-1) t^{r}} \\
\bar{\Delta}_{j} & =\frac{f}{t}+\frac{1}{t} \int^{t} 24 A_{1}^{2} g_{j} \sum_{r=3}^{\infty} \frac{c_{r-1}}{(r-1) s^{r-1}}=-24 A_{1}^{2} g_{j} \sum_{r=3}^{\infty} \frac{c_{r-1}}{(r-1)(r-2) t^{r-1}} \\
& =\frac{-12 A_{1}^{2} g_{j} d_{2}}{t^{2}}-24 A_{1}^{2} g_{j} \sum_{r=3}^{\infty} \frac{c_{r}}{r(r-1) t^{r}},
\end{aligned}
$$

where we have chosen $e$ and $f$ to cancel the integral term at the lower limit. It is easy to see that all the series converge for all values of $t$, so the formal series solution is an actual (power series) solution. This solution can be rotated back to $\Delta$, and we have

$$
\left(\begin{array}{c}
\Delta_{j} \\
\Delta_{N+j}
\end{array}\right)=\left(\begin{array}{c}
O(1 / t) \\
O\left(1 / t^{2}\right)
\end{array}\right)
$$

The choice of $d_{2}=0$ offers an interesting special case:

$$
\begin{aligned}
\bar{\Delta}_{1} & =-\frac{A_{1}}{3 t}+\frac{3 A_{1}^{3} g_{1}}{t^{3}}+\sum_{r=4}^{\infty} \frac{c_{r}}{t^{r}} \\
\bar{\Delta}_{2} & =-\frac{24 A_{1}^{2} g_{1} g_{2}}{t^{3}}+24 A_{1}^{2}\left(b g_{1} \sum_{r=4}^{\infty} \frac{c_{r-1}}{(r-1)(r-2) t^{r}}-g_{2} \sum_{r=4}^{\infty} \frac{c_{r}}{(r-1)^{2} t^{r}}\right) \\
\bar{\Delta}_{j} & =-\frac{16 A_{1}^{1} g_{1} g_{j}(b-1)}{t^{3}}-24 A_{1}^{2} g_{j}(b-1) \sum_{r=4}^{\infty} \frac{c_{r}}{r(r-1) t^{r}} \\
\bar{\Delta}_{N+1} & =\frac{48 A_{1}^{5} g_{1}^{2}}{t^{4}}+\sum_{r=5}^{\infty} \frac{d_{r}}{t^{r}} \\
\bar{\Delta}_{N+2} & =\frac{32 A_{1}^{5} g_{1}^{2}}{t^{4}}+\sum_{r=5}^{\infty} \frac{e_{r}}{t^{r}} \\
\bar{\Delta}_{3 N+j} & =\frac{4 A_{1}^{3} g_{1}}{t^{4}}+\sum_{r=4}^{\infty} \frac{c_{r-1}}{(r-1) t^{r}},
\end{aligned}
$$

and we have

$$
\left(\begin{array}{c}
\Delta_{j} \\
\Delta_{N+j}
\end{array}\right)=\left(\begin{array}{c}
O(1 / t) \\
O\left(1 / t^{4}\right)
\end{array}\right)
$$

\subsection{Return to the Uncompactified Problem}

Inspired by the presence of a $\log$ term in the solution (4.2.4 - 4.2.6) for $\Delta$, we suppose there is a solution $x=\bar{x}+\Delta$, where $\bar{x}$ is given in (3.3.12) in closed form as

$$
\begin{aligned}
\bar{x}_{i} & =2 \frac{x_{i}(0)+x_{N+i}(0) t}{1-R(0)^{2}+\sqrt{\left(1+R(0)^{2}\right)^{2}+4\left(2 h(0) t+A(0) t^{2}\right)}} \\
\bar{x}_{N+i} & =2 \frac{x_{N+i}(0)}{1-R(0)^{2}+\sqrt{\left(1+R(0)^{2}\right)^{2}+4\left(2 h(0) t+A(0) t^{2}\right)}}
\end{aligned}
$$

and has a power series in $1 / t$, and $\Delta$ tends to 0 as $t \rightarrow \infty$, and $\Delta=\left(\begin{array}{c}O(\log t / t) \\ O\left(\log t / t^{2}\right)\end{array}\right)$. Then we can write

$$
x=\left(\begin{array}{c}
x^{*}+e \log t / t+g / t \\
x^{*} / t+f \log t / t^{2}+h / t^{2}
\end{array}\right)+\left(\begin{array}{c}
o(1 / t) \\
o\left(1 / t^{2}\right)
\end{array}\right)
$$


where $e, f, g$, and $h$ are $3 N$-vectors. If we would compactify $y$ of the form

$$
y=\left(\begin{array}{c}
q \\
\dot{q}
\end{array}\right)=\left(\begin{array}{c}
a t+b \log t+c+\delta \\
a+b / t+\dot{\delta}
\end{array}\right)
$$

where $\delta \in C^{2}\left[t_{0}, \infty\right)$ has certain properties, in particular that $\delta=o(1)$ as $t \rightarrow \infty$, then the result is of the form (4.3.1). This can be seen as we compactify (4.3.2)

$$
\begin{aligned}
& q_{i}=a_{i} t+b_{i} \log t+c_{i}+\delta_{i} \\
& \dot{q}_{i}=a_{i}+\frac{b_{i}}{t}+\dot{\delta}_{i}
\end{aligned}
$$

per the instructions in Section 1.2. First let us define the shorthand

$$
A=\sqrt{\sum_{i=1}^{N} a_{i}^{\dagger} a_{i}}
$$

and we proceed as follows:

$$
\begin{gathered}
r^{2}=A^{2} t^{2}+2 \sum_{i=1}^{N} a_{i}^{\dagger} b_{i} t \log t+2 \sum_{i=1}^{N} a_{i}^{\dagger} c_{i} t+o(t) \\
1+4 r^{2}=4 A^{2} t^{2}\left(1+2 \frac{\frac{\log t}{t} \sum_{i=1}^{N} a_{i}^{\dagger} b_{i}+\frac{1}{t} \sum_{i=1}^{N} a_{i}^{\dagger} c_{i}}{A^{2}}+o\left(\frac{1}{t}\right)\right) \\
\sqrt{1+4 r^{2}}=2 A t\left(1+2 \frac{\frac{\log t}{t} \sum_{i=1}^{N} a_{i}^{\dagger} b_{i}+\frac{1}{t} \sum_{i=1}^{N} a_{i}^{\dagger} c_{i}}{A^{2}}+o\left(\frac{1}{t}\right)\right)^{1 / 2} \\
=2 A t\left(1+\frac{\frac{\log t}{t} \sum_{i=1}^{N} a_{i}^{\dagger} b_{i}+\frac{1}{t} \sum_{i=1}^{N} a_{i}^{\dagger} c_{i}}{A^{2}}+o\left(\frac{1}{t}\right)\right) \\
\sqrt{1+4 r^{2}}=2 A t\left(1+\frac{\frac{\log t}{t} \sum_{i=1}^{N} a_{i}^{\dagger} b_{i}+\frac{1}{t} \sum_{i=1}^{N} a_{i}^{\dagger} c_{i}}{A^{2}}+\frac{1}{2 A t}+o\left(\frac{1}{t}\right)\right) \\
=2 A t
\end{gathered}
$$

So the compactified trajectory has the asymptotic approximation

$$
\begin{aligned}
& x=\frac{2}{1+\sqrt{1+4 r^{2}}}\left(\begin{array}{c}
q \\
\dot{q}
\end{array}\right) \\
& =\left[\frac{1}{A t}\left(1-\frac{\frac{\log t}{t} \sum_{i=1}^{N} a_{i}^{\dagger} b_{i}+\frac{1}{t} \sum_{i=1}^{N} a_{i}^{\dagger} c_{i}+\frac{1}{2 t} A}{A^{2}}\right)+o\left(\frac{1}{t^{2}}\right)\right]\left(\begin{array}{c}
a t+b \log t+c+o(1) \\
a+\frac{b}{t}+o\left(\frac{1}{t}\right)
\end{array}\right) \\
& =\frac{1}{A}\left(\begin{array}{c}
a+b \frac{\log t}{t}+c \frac{1}{t} \\
a \frac{1}{t}+b \frac{1}{t^{2}}
\end{array}\right)-\frac{\sum_{i=1}^{N} a_{i}^{\dagger} b_{i}}{A^{3}}\left(\begin{array}{c}
a \frac{\log t}{t} \\
a \frac{\log t}{t^{2}}
\end{array}\right)-\frac{\sum_{i=1}^{N} a_{i}^{\dagger} c_{i}+\frac{1}{2} A}{A^{3}}\left(\begin{array}{c}
a \frac{1}{t} \\
a \frac{1}{t^{2}}
\end{array}\right)+\left(\begin{array}{c}
o\left(\frac{1}{t}\right) \\
o\left(\frac{1}{t^{2}}\right)
\end{array}\right) \\
& x=\left(\begin{array}{c}
x^{*}+\frac{1}{A}\left(b-\sum_{i=1}^{N} x_{i}^{* \dagger} b_{i} x^{*}\right) \frac{\log t}{t}+\frac{1}{A}\left(c-\left[\sum_{i=1}^{N} x_{i}^{* \dagger} c_{i}+\frac{1}{2}\right] x^{*}\right) \frac{1}{t}+o\left(\frac{1}{t}\right) \\
\frac{x^{*}}{t}-\frac{1}{A} \sum_{i=1}^{N} x_{i}^{* \dagger} b_{i} x^{*} \frac{\log t}{t^{2}}+\frac{1}{A}\left(b-\left[\sum_{i=1}^{N} x_{i}^{* \dagger} c_{i}+\frac{1}{2}\right] x^{*}\right) \frac{1}{t^{2}}+o\left(\frac{1}{t^{2}}\right)
\end{array}\right),
\end{aligned}
$$

with the $3 N$-vector $x^{*}=\frac{1}{A} a$. Note (4.3.1) has the form of

and $x$ can be seen to approach

$$
x=\bar{x}+\Delta,
$$

$$
\left(\begin{array}{c}
x^{*} \\
0_{3 N}
\end{array}\right)=\frac{1}{A}\left(\begin{array}{c}
a_{i} \\
0_{3 N}
\end{array}\right)
$$


a critical point on the boundary.

It would be nice if we could identify the decomposition of $x$ into $\bar{x}$ and $\Delta$; clearly $e$ and $f$, the second terms in each part of (4.3.3) present the leading term of $\Delta$, but $\Delta$ can be expected to have terms in $1 / t$ and $1 / t^{2}$, as well, so it isn't possible to separate the third term into pieces from $\bar{x}$ and $\Delta$.

The leading terms of (4.3.3) are

$$
x=\left(\begin{array}{c}
x^{*}+\frac{\log t}{t} e+\frac{1}{t} g+\frac{\log t}{t^{2}} r+\frac{1}{t^{2}} s \\
\frac{1}{t} x^{*}+\frac{\log t}{t^{2}} f+\frac{1}{t^{2}} h
\end{array}\right)+o\left(\frac{1}{t^{2}}\right),
$$

where $e, f, g, h, r$, and $s$ are $3 N$-vectors

$$
\begin{aligned}
e & =\frac{1}{A}\left(b-\sum_{i=1}^{N} x_{i}^{* \dagger} b_{i} x^{*}\right) \\
f & =-\frac{1}{A} \sum_{i=1}^{N} x_{i}^{* \dagger} b_{i} x^{*} \\
g & =\frac{1}{A}\left(c-\left[\sum_{i=1}^{N} x_{i}^{* \dagger} c_{i}+\frac{1}{2}\right] x^{*}\right) \\
h & =\frac{1}{A}\left(b-\left[\sum_{i=1}^{N} x_{i}^{* \dagger} c_{i}+\frac{1}{2}\right] x^{*}\right) .
\end{aligned}
$$

Note that $x$ has the form $\bar{x}+\Delta$, for the choice of $\Delta$ from solving a version of the $J_{0}$ problem where we found a term $\frac{\log t}{t}$ in Section 4.2. Also note that the $3 N$-vectors $x^{*}$ and $e$ are orthogonal:

$$
\sum_{i=1}^{N} x_{i}^{* \dagger} e_{i}=\frac{1}{A}\left(\sum_{i=1}^{N} x_{i}^{* \dagger} b_{i}-\sum_{i=1}^{N} x_{i}^{* \dagger} b_{i}\right)=0 .
$$

On the other hand, $g$ is not orthogonal to $x^{*}$

$$
\sum_{i=1}^{N} x_{i}^{* \dagger} g_{i}=\frac{1}{A}\left(\sum_{i=1}^{N} x_{i}^{* \dagger} c_{i}-\sum_{i=1}^{N} x_{i}^{* \dagger} c_{i}-\frac{1}{2}\right)=-\frac{1}{2 A} .
$$




\section{CHAPTER 5}

\section{Solutions That Escape}

This Chapter establishes the main theorem of the dissertation, that solutions of Bohlin's form (5.0.1) do indeed exist for all time. The existence is demonstrated of an open set of initial conditions through which pass solutions without singularities, to Newton's gravitational equations in $\mathbb{R}^{3}$ on a semi-infinite interval in forward time, for which every pair of particles separates like At, $A>0$, as $t \rightarrow \infty$. The solutions are constructible as series with rapid uniform convergence and their asymptotic behavior to any order is prescribed. This family of solutions depends on $6 N$ parameters subject to certain constraints. The key to the result is converting (1.0.1) the differential equation for q into an integral equation for $\delta$ and showing that integral equation has a solution. The main theorem is proven by a chain of lemmas. We substitute for $q_{i}$ in (1.0.1), obtaining an equation for $\ddot{\delta}_{i}$. As we are seeking $\delta_{i}=o(1)$ as $t \rightarrow \infty$, we match terms $\geq O(1)$, yielding a formula for $b_{i}$. We will decompose the right hand side of $\ddot{\delta}_{i}$ into $P 1_{i}+P 2_{i}$, where each $P 1_{i}$ is independent of $\delta$. We convert the differential equation into an integral equation for $\delta_{i}$. We propose an iterative solution, the limit of the sequence given by

$$
\delta_{i}[n+1]=\iint\left(P 1_{i}+P 2_{i}(\delta[n])\right)
$$

We establish estimates for $P 1_{i}$ and $P 2_{i}$, and then prove via induction that each $\left\{\delta_{i}[n]\right\}_{n=1}^{\infty}$ is uniformly bounded and Cauchy, and thus converges to a solution $\delta_{i}$. As will be apparent, the proof is identical for the planar problem. The results of this Chapter were published in 2017 [43].

THEOREM 29. Given any set of masses $m_{i}>0$ and constant 3-vectors $a_{i}$ and $c_{i}, i=1, \ldots, N$, satisfying $\left\|a_{j}-a_{i}\right\| \neq 0, i \neq j$, the differential system (1.0.1) possesses unique vector solutions

$$
q_{i}=a_{i} t+b_{i} \log t+c_{i}+\delta_{i}(t) i=1, \ldots, N,
$$

on a semi-infinite interval $\left[t_{5}, \infty\right)$ where $t_{5} \geq 1$, and $q_{i} \in C^{\infty}\left[t_{5}, \infty\right)$. The $3 N$ coefficients $b_{i}$ are uniquely determined by the $3 N$ coefficients $a_{i}$ as follows

$$
b_{i}=\sum_{j \neq i} \frac{m_{j}\left(a_{i}-a_{j}\right)}{\left\|a_{i}-a_{j}\right\|^{3}}, i=1, \ldots, N .
$$

Each vector $\delta_{i}(t)$ is approximated to any level of accuracy by a sequence $\delta_{i}[n]$ and computed successively by certain iterations, and

$$
\delta_{i}(t)-\delta_{i}[n](t)=O\left(\frac{K_{4}^{n}}{[(n+1) !]^{2}} \frac{\log t}{t^{n+1}}\right),
$$

uniformly for $t \in\left[t_{5}, \infty\right)$ as $n \rightarrow \infty$, where $K_{4}>0$ will be defined in the sequel.

Moreover, the following asymptotic relations hold with $a_{i} \neq 0_{3}$,

$$
q_{i}=\left\{I_{3}+\triangle_{i}\right\}\left[a_{i} t+b_{i} \log t+c_{i}+\delta_{i}[n](t)\right], \text { where } \triangle_{i}=O\left(\frac{K_{4}^{n}}{[(n+1) !]^{2}} \frac{\log t}{t^{n+2}}\right),
$$

uniformly for $t \in\left[t_{5}, \infty\right)$ as $n \rightarrow \infty$, where each $\Delta_{i}$ is a 3 -by-3 matrix. If one $a_{i}=0_{3}$, but $b_{i} \neq 0_{3}$, the resulting asymptotic formula will be instead

$$
q_{i}=\left\{I_{3}+\triangle_{i}\right\}\left[b_{i} \log t+c_{i}+\delta_{i}[n](t)\right], \text { where } \triangle_{i}=O\left(\frac{K_{4}^{n}}{[(n+1) !]^{2}} \frac{1}{t^{n+2}}\right),
$$

uniformly for $t \in\left[t_{5}, \infty\right)$ as $n \rightarrow \infty$. There are also solutions with one $a_{i}=0_{3}$ and $b_{i}=0_{3}$, and the asymptotic formula 


$$
q_{i}=\left\{I_{3}+\triangle_{i}\right\}\left[c_{i}+\delta_{i}[n](t)\right], \text { where } \triangle_{i}=O\left(\frac{K_{4}^{n}}{[(n+1) !]^{2}} \frac{1}{t^{n+2}}\right),
$$

uniformly for $t \in\left[t_{5}, \infty\right)$ as $n \rightarrow \infty$. In other words, either all particles escape to infinity, or precisely one particle reaches the rest point $c_{i}$ as $t \rightarrow \infty$ while the rest of the particles escape to infinity.

The remainder of this Chapter continues as follows. In Section 5.1 we define $P 1_{i}$ and $P 2_{i}$ and convert our differential system (1.0.1) into an integral system of equations. In Section 5.2, we obtain certain estimates on components of the integral equations. In Section 5.3 we obtain a sequence of successive approximations that converge to the desired solutions. We conclude with Section 5.4, where we obtain robust asymptotic approximations to various quantities associated with the $N$-body problem and compare them to results of Pollard and Saari and their collaborators.

\subsection{Seeking solutions with escaping trajectories}

Given constant 3 -vectors $a_{i}, b_{i}, c_{i}, i=1, \ldots, N$, for $t>1$, let $f_{i}=f_{i}(t)$ be defined

$$
f_{i}=f_{i}(t):=a_{i} t+b_{i} \log t+c_{i} \text {, so that } q_{i}=f_{i}(t)+\delta_{i}(t)=a_{i} t+b_{i} \log t+c_{i}+\delta_{i}(t) .
$$

Thus, the equation (1.0.1) becomes

$$
\ddot{q}_{i}=-b_{i} t^{-2}+\ddot{\delta}_{i}(t)=\sum_{j \neq i} \frac{m_{j}\left(q_{j}-q_{i}\right)}{\left\|q_{i}-q_{j}\right\|^{3}} .
$$

Our next goal is to substitute $q_{i}=a_{i} t+b_{i} \log t+c_{i}+\delta_{i}(t)$ in (5.1.2) and obtain a differential system for $\delta_{i}(t)$ free of the symbol $q$ that will guarantee solutions to (5.1.2) with the desired properties mentioned above. First, the substitution gives us

$$
-b_{i} t^{-2}+\ddot{\delta}_{i}=\sum_{j \neq i} \frac{m_{j}\left[\left(a_{j}-a_{i}\right) t+\left(b_{j}-b_{i}\right) \log t+\left(c_{j}-c_{i}\right)+\delta_{j}(t)-\delta_{i}(t)\right]}{\left\|\left(a_{j}-a_{i}\right) t+\left(b_{j}-b_{i}\right) \log t+\left(c_{j}-c_{i}\right)+\delta_{j}(t)-\delta_{i}(t)\right\|^{3}} .
$$

Our purpose is to bring the second order differential system for $\delta_{i}$ to the following form

$$
\ddot{\delta}_{i}=P 1_{i}(t)+P 2_{i}(\delta, t)
$$

where each $P 1_{i}$ is independent of $\delta$ and tends to zero as $t \rightarrow \infty$, and each $P 2_{i}$ is dependent on $\delta$. In addition, we want that if each $\delta_{i}(t)=$ the zero vector in $\mathbb{R}^{3}$, then so does every $P 2_{i}$.

Since we can express each $\left\|q_{i}-q_{j}\right\|^{2}$ as

$$
\times\left\{1+\frac{\left.2\left(a_{j}-a_{i}\right)^{\dagger}\left[\left(b_{j}-b_{i}\right) \log t+c_{j}-c_{i}+\delta_{j}(t)-\delta_{i}\right) \|^{2} t^{2}(t)\right]+\left\|\left(b_{j}-b_{i}\right) \log t+c_{j}-c_{i}+\delta_{j}(t)-\delta_{i}(t)\right\|^{2}}{\left\|a_{j}-a_{i}\right\|^{2} t^{2}}\right\},
$$

we can write each summand as

$$
\begin{gathered}
\frac{m_{j}\left[\left(a_{j}-a_{i}\right)+\frac{1}{t}\left[\left(b_{j}-b_{i}\right) \log t+\left(c_{j}-c_{i}\right)+\delta_{j}(t)-\delta_{i}(t)\right]\right]}{\left\|\left(a_{j}-a_{i}\right)\right\|^{3} t^{2}} \\
\times\left\{1+\frac{2\left(a_{j}-a_{i}\right)^{\dagger}\left[\left(b_{j}-b_{i}\right) \log t+c_{j}-c_{i}+\delta_{j}(t)-\delta_{i}(t)\right]+\left\|\left(b_{j}-b_{i}\right) \log t+c_{j}-c_{i}+\delta_{j}(t)-\delta_{i}(t)\right\|^{2}}{\left\|a_{j}-a_{i}\right\|^{2} t^{2}}\right\}^{-3 / 2} .
\end{gathered}
$$

Multiplying $t^{2}$ times each side of (5.1.3) yields

$$
\begin{gathered}
-b_{i}+\ddot{\delta}_{i} t^{2}=\sum_{j \neq i} \frac{m_{j}\left[\left(a_{j}-a_{i}\right)+\frac{1}{t}\left[\left(b_{j}-b_{i}\right) \log t+\left(c_{j}-c_{i}\right)+\delta_{j}(t)-\delta_{i}(t)\right]\right]}{\left\|\left(a_{j}-a_{i}\right)\right\|^{3}} \\
\times\left\{1+\frac{2\left(a_{j}-a_{i}\right)^{\dagger}\left[\left(b_{j}-b_{i}\right) \log t+c_{j}-c_{i}+\delta_{j}(t)-\delta_{i}(t)\right]+\left\|\left(b_{j}-b_{i}\right) \log t+c_{j}-c_{i}+\delta_{j}(t)-\delta_{i}(t)\right\|^{2}}{\left\|a_{j}-a_{i}\right\|^{2} t^{2}}\right\}^{-3 / 2} .
\end{gathered}
$$

Taking the limit as $t \rightarrow \infty$, assuming $\ddot{\delta}_{i}=o\left(1 / t^{2}\right)$ as $t \rightarrow \infty$, we see we must have the $N$ vector identities: 


$$
b_{i}=-\sum_{j \neq i} \frac{m_{j}\left(a_{j}-a_{i}\right)}{\left\|a_{j}-a_{i}\right\|^{3}}, i=1, \ldots, N
$$

where of course we assume that

$$
\left\|a_{j}-a_{i}\right\| \neq 0, i \neq j, i, j=1, \ldots, N .
$$

It is evident that with the choice of $b_{i}$ as given in (5.1.5) the equation (5.1.3) becomes

$$
\ddot{\delta}_{i}=\sum_{j \neq i} \frac{m_{j}\left[f_{j}-f_{i}+\delta_{j}-\delta_{i}\right]}{\left\|f_{j}-f_{i}+\delta_{j}-\delta_{i}\right\|^{3}}-\frac{1}{t^{2}} \sum_{j \neq i} \frac{m_{j}\left(a_{j}-a_{i}\right)}{\left\|a_{j}-a_{i}\right\|^{3}} .
$$

While (5.1.7) is a decomposition into components dependent and free of $\delta$, a rearrangement as follows will give us more useful estimates and better control.

Notice that by adding and subtracting the term $\sum_{j \neq i} \frac{m_{j}\left[f_{j}-f_{i}\right]}{\left\|f_{j}-f_{i}\right\|^{3}}$ on the right hand side of (5.1.7) we obtain

$$
\ddot{\delta}_{i}=\sum_{j \neq i} \frac{m_{j}\left[f_{j}-f_{i}+\delta_{j}-\delta_{i}\right]}{\left\|f_{j}-f_{i}+\delta_{j}-\delta_{i}\right\|^{3}}-\sum_{j \neq i} \frac{m_{j}\left[f_{j}-f_{i}\right]}{\left\|f_{j}-f_{i}\right\|^{3}}+\sum_{j \neq i} \frac{m_{j}\left[f_{j}-f_{i}\right]}{\left\|f_{j}-f_{i}\right\|^{3}}-\frac{1}{t^{2}} \sum_{j \neq i} \frac{m_{j}\left(a_{j}-a_{i}\right)}{\left\|a_{j}-a_{i}\right\|^{3}} .
$$

We now define

$$
\begin{aligned}
P 1_{i}(t): & =\sum_{j \neq i} m_{j}\left(\frac{f_{j}-f_{i}}{\left\|f_{j}-f_{i}\right\|^{3}}-\frac{a_{j}-a_{i}}{t^{2}\left\|a_{j}-a_{i}\right\|^{3}}\right) \\
P 2_{i}(\delta(t), t): & =\sum_{j \neq i} m_{j}\left(\frac{f_{j}-f_{i}+\delta_{j}-\delta_{i}}{\left\|f_{j}-f_{i}+\delta_{j}-\delta_{i}\right\|^{3}}-\frac{f_{j}-f_{i}}{\left\|f_{j}-f_{i}\right\|^{3}}\right) .
\end{aligned}
$$

This conforms to (5.1.4). Note that if each $\delta_{i}(t)=$ the zero vector in $\mathbb{R}^{3}$, then so does every $P 2_{i}(\delta(t), t)$. We summarize the above discussion in the following lemma.

Lemma 30. Assume (5.1.6) and (5.1.5). If each $\delta_{i}(t)$ is a solution of (5.1.4) on an interval $\left[t_{0}, \infty\right)$ tending to 0 : each $\delta_{i}=o(1)$ and $\ddot{\delta}_{i}=o\left(1 / t^{2}\right)$ as $t \rightarrow \infty$, then $q_{i}(t)$ is a solution of (5.1.2).

If $P 1$ and $P 2$ are well enough behaved, the double integral of (5.1.4) will be well-defined:

$$
\delta_{i}=\int_{t}^{\infty} \int_{s}^{\infty}\left(P 1_{i}(u)+P 2_{i}(\delta(u), u)\right) d u d s .
$$

Henceforth, we focus on this integral equation; we will need estimates for $P 1_{i}$ and $P 2_{i}$.

\subsection{Estimates for $P 1$ and $P 2$}

In this Section we give several lemmas to demonstrate that there is an interval $\left(t_{4}, \infty\right)$ on which: if $\left\|\delta_{i}(t)\right\|<1$, then for certain constants $K_{1}, K_{2}, K_{3}$, we have estimates

$$
\left\|P 1_{i}\right\| \leq K_{1} \log t / t^{3}<1 \text {, and }\left\|P 2_{i}\right\| \leq K_{2} / t^{3} .
$$

Moreover, if $w$ is an upper bound for all the $\left\|\delta_{i}-\eta_{i}\right\|$, then in addition $\left\|P 2_{i}(\delta)-P 2_{i}(\eta)\right\| \leq K_{3} w / t^{3}$.

Each denominator of (5.1.5) is the cube of

$$
A_{i j}:=\left\|a_{j}-a_{i}\right\| .
$$

Similarly, we define

$$
F_{i j}:=\left\|f_{j}-f_{i}\right\|=\left\|\left(a_{j}-a_{i}\right) t+\left(b_{j}-b_{i}\right) \log t+c_{j}-c_{i}\right\| .
$$

Then

$$
\begin{aligned}
F_{i j}^{2}= & \left\|a_{j}-a_{i}\right\|^{2} t^{2}+2 t\left(a_{j}-a_{i}\right)^{\dagger}\left(\left(b_{j}-b_{i}\right) \log t+c_{j}-c_{i}\right)+\left\|\left(b_{j}-b_{i}\right) \log t+c_{j}-c_{i}\right\|^{2} \\
& =A_{i j}^{2} t^{2}+2 t\left(a_{j}-a_{i}\right)^{\dagger}\left[\left(b_{j}-b_{i}\right) \log t+c_{j}-c_{i}\right]+\left\|\left(b_{j}-b_{i}\right) \log t+c_{j}-c_{i}\right\|^{2} .
\end{aligned}
$$

We assume that (5.1.6) holds, and we factor out the anticipated leading term in (5.2.1) as follows 


$$
F_{i j}^{2}=A_{i j}^{2} t^{2}\left\{1+\frac{2 t\left(a_{j}-a_{i}\right)^{\dagger}\left[\left(b_{j}-b_{i}\right) \log t+c_{j}-c_{i}\right]+\left\|\left(b_{j}-b_{i}\right) \log t+c_{j}-c_{i}\right\|^{2}}{A_{i j}^{2} t^{2}}\right\} .
$$

We can use the following abbreviation for a lengthy expression in (5.2.2)

$$
G_{i j}=\frac{2}{t}\left(a_{j}-a_{i}\right)^{\dagger}\left[\left(b_{j}-b_{i}\right) \log t+c_{j}-c_{i}\right]+\frac{1}{t^{2}}\left\|\left(b_{j}-b_{i}\right) \log t+c_{j}-c_{i}\right\|^{2},
$$

so that

$$
F_{i j}^{2}=A_{i j}^{2} t^{2}\left(1+\frac{G_{i j}}{A_{i j}^{2}}\right)
$$

5.2.1. Estimating $P 1$. Our next goal is to provide a simpler representation for $P 1_{i}(t)$ as $t \rightarrow \infty$. To that end we first determine an approximation for $F_{i j}$. Notice that $\left(b_{j l}-b_{i l}\right) \log t+c_{j l}-c_{i l}$ appears in (5.2.3) as a factor in each of the two terms. Also observe that for $t>1$

$$
\left(b_{j}-b_{i}\right) \log t+c_{j}-c_{i}=\log t\left[\left(b_{j}-b_{i}\right)+\frac{c_{j}-c_{i}}{\log t}\right] .
$$

Utilize (5.2.4) in (5.2.3) to obtain

$$
G_{i j}=\frac{\log t}{t}\left\{2\left(a_{j}-a_{i}\right)^{\dagger}\left[\left(b_{j}-b_{i}\right)+\frac{c_{j}-c_{i}}{\log t}\right]+\frac{\log t}{t}\left\|\left(b_{j}-b_{i}\right)+\frac{c_{j}-c_{i}}{\log t}\right\|^{2}\right\} .
$$

By the Schwarz inequality

$$
\left|\left(a_{j}-a_{i}\right)^{\dagger}\left[\left(b_{j}-b_{i}\right)+\frac{c_{j}-c_{i}}{\log t}\right]\right| \leq A_{i j}\left\|\left(b_{j}-b_{i}\right)+\frac{c_{j}-c_{i}}{\log t}\right\|,
$$

so that

$$
\frac{\left|G_{i j}\right|}{A_{i j}^{2}} \leq \frac{\log t}{A_{i j} t}\left\{2\left\|\left(b_{j}-b_{i}\right)+\frac{c_{j}-c_{i}}{\log t}\right\|+\frac{\log t}{A_{i j} t}\left\|\left(b_{j}-b_{i}\right)+\frac{c_{j}-c_{i}}{\log t}\right\|^{2}\right\} .
$$

Now by the triangle inequality

$$
\frac{\left|G_{i j}\right|}{A_{i j}^{2}} \leq \frac{\log t}{\underline{A} t}\left\{2\left(2 \hat{b}+\frac{2 \hat{c}}{\log t}\right)+\frac{\log t}{\underline{A} t}\left(2 \hat{b}+\frac{2 \hat{c}}{\log t}\right)^{2}\right\} \leq \frac{4 \log t}{\underline{A} t}\left\{\hat{b}+\frac{\hat{c}}{\log t}+\frac{\log t}{\underline{A} t}\left(\hat{b}+\frac{\hat{c}}{\log t}\right)^{2}\right\}
$$

where $\hat{b}=\max _{k}\left\|b_{k}\right\|, \hat{c}=\max _{k}\left\|c_{k}\right\|, \underline{A}=\min _{i, j} A_{i j}>0$.

Lemma 31. Assume (5.1.6) and let $b$ be given by (5.1.5). Then

$$
F_{i j}^{2}=A_{i j}^{2} t^{2}\left(1+\frac{G_{i j}}{A_{i j}^{2}}\right), \quad G_{i j} \rightarrow 0,
$$

and for any constant $K_{0}>4 \hat{b} / \underline{A}$ and for any $\rho, 0<\rho<1$, there exists $t_{0}=t_{0}(\rho)>1$ independent of $i$ and $j$, such that for $t \geq t_{0}$

$$
\left|\frac{G_{i j}}{A_{i j}^{2}}\right| \leq K_{0} \frac{\log t}{t} \leq \rho<1 .
$$

Proof. By elementary calculus $(\log t)^{-1} \rightarrow 0$ and $t^{-1} \log t \rightarrow 0$ as $t \rightarrow \infty$; so for sufficiently large $t$, the rest of the terms in the estimate (5.2.6) can be made as small as desired. In particular, for any $K_{0}>4 \hat{b} / \underline{A}$ there exists $\hat{t}_{0}$, such that for $t \geq \hat{t}_{0}$,

$$
\frac{4}{\underline{A}}\left\{\hat{b}+\frac{\hat{c}}{\log t}+\frac{\log t}{\underline{A} t}\left(\hat{b}+\frac{\hat{c}}{\log t}\right)^{2}\right\} \leq K_{0} .
$$

Moreover, for any $\rho<1$ there exists $\bar{t}_{0}$, such that for $t \geq \bar{t}_{0}, \frac{\log t}{t} \leq \frac{\rho}{K_{0}}$, so (5.2.8) follows with $t_{0}:=$ $\max \left\{\bar{t}_{0}, \hat{t}_{0}\right\}$. 
We are ready now to estimate the entire term $P 1(t)$. We have with the aid of Lemma 2

$$
\begin{gathered}
P 1_{i}(t)=\sum_{j \neq i} m_{j}\left[\frac{f_{j}-f_{i}}{F_{i j}^{3}}-\frac{a_{j}-a_{i}}{t^{2} A_{i j}^{3}}\right]=\sum_{j \neq i} m_{j}\left[\frac{\left(a_{j}-a_{i}\right) t+\left(b_{j}-b_{i}\right) \log t+c_{j}-c_{i}}{\left.A_{i j}^{3} t^{3}\left(1+\frac{G_{i j}}{A_{i j}^{2}}\right)^{\frac{3}{2}}-\frac{a_{j}-a_{i}}{t^{2} A_{i j}^{3}}\right]}\right. \\
=\sum_{j \neq i} m_{j}\left[\left(1+\frac{G_{i j}}{A_{i j}^{2}}\right)^{-\frac{3}{2}} \frac{\left(a_{j}-a_{i}\right) t+\left(b_{j}-b_{i}\right) \log t+c_{j}-c_{i}}{A_{i j}^{3} t^{3}}-\frac{a_{j}-a_{i}}{t^{2} A_{i j}^{3}}\right] \\
=\sum_{j \neq i} m_{j}\left[\left[\left(1+\frac{G_{i j}}{A_{i j}^{2}}\right)^{-\frac{3}{2}}-1\right] \frac{\left(a_{j}-a_{i}\right) t+\left(b_{j}-b_{i}\right) \log t+c_{j}-c_{i}}{A_{i j}^{3} t^{3}}\right. \\
\left.+\frac{\left(a_{j}-a_{i}\right) t+\left(b_{j}-b_{i}\right) \log t+c_{j}-c_{i}}{A_{i j}^{3} t^{3}}-\frac{a_{j}-a_{i}}{t^{2} A_{i j}^{3}}\right] .
\end{gathered}
$$

Simplifying,

$$
P 1_{i}(t)=\sum_{j \neq i} m_{j}\left[\left[\left(1+\frac{G_{i j}}{A_{i j}^{2}}\right)^{-\frac{3}{2}}-1\right] \frac{\left(a_{j}-a_{i}\right) t+\left(b_{j}-b_{i}\right) \log t+c_{j}-c_{i}}{A_{i j}^{3} t^{3}}+\frac{\left(b_{j}-b_{i}\right) \log t+c_{j}-c_{i}}{A_{i j}^{3} t^{3}}\right] .
$$

We focus on the expression $\frac{\left(b_{j}-b_{i}\right) \log t+c_{j}-c_{i}}{A_{i j}^{3} t^{3}}$ occurring in $P 1_{i}(t)$ and notice that

$$
\frac{\left(b_{j}-b_{i}\right) \log t+c_{j}-c_{i}}{A_{i j}^{3} t^{3}}=\frac{\log t}{A_{i j}^{3} t^{3}}\left[b_{j}-b_{i}+\frac{c_{j}-c_{i}}{\log t}\right] .
$$

Therefore, we have

$$
\frac{\left(a_{j}-a_{i}\right) t+\left(b_{j}-b_{i}\right) \log t+c_{j}-c_{i}}{A_{i j}^{3} t^{3}}=\frac{a_{j}-a_{i}}{A_{i j}^{3} t^{2}}+\frac{\left(b_{j}-b_{i}\right) \log t+c_{j}-c_{i}}{A_{i j}^{3} t^{3}} .
$$

With the aid of (5.2.10) we get

$$
\begin{gathered}
\frac{a_{j}-a_{i}}{A_{i j}^{3} t^{2}}+\frac{\left(b_{j}-b_{i}\right) \log t+c_{j}-c_{i}}{A_{i j}^{3} t^{3}}=\frac{a_{j}-a_{i}}{A_{i j}^{3} t^{2}}+\frac{\log t}{A_{i j}^{3} t^{3}}\left[b_{j}-b_{i}+\frac{c_{j}-c_{i}}{\log t}\right] \\
=\frac{a_{j}-a_{i}+\frac{\log t}{t}\left[b_{j}-b_{i}+\frac{c_{j}-c_{i}}{\log t}\right]}{A_{i j}^{3} t^{2}} .
\end{gathered}
$$

Finally we rewrite

$$
\begin{gathered}
P 1_{i}(t)=\frac{1}{t^{2}} \sum_{j \neq i} \frac{m_{j}}{A_{i j}^{3}}\left\{\left[\left(1+\frac{G_{i j}}{A_{i j}^{2}}\right)^{-\frac{3}{2}}-1\right]\left(a_{j}-a_{i}+\frac{\log t}{t}\left[b_{j}-b_{i}+\frac{c_{j}-c_{i}}{\log t}\right]\right)\right. \\
\left.+\frac{\log t}{t}\left[b_{j}-b_{i}+\frac{c_{j}-c_{i}}{\log t}\right]\right\} .
\end{gathered}
$$

We start with the bound

$$
\left\|P 1_{i}(t)\right\| \leq \frac{1}{t^{2}} \sum_{j \neq i} \frac{m_{j}}{A_{i j}^{3}}
$$

$$
\times\left\{\left|\left(1+\frac{G_{i j}}{A_{i j}^{2}}\right)^{-\frac{3}{2}}-1\right|\left\|a_{j}-a_{i}+\frac{\log t}{t}\left[b_{j}-b_{i}+\frac{c_{j}-c_{i}}{\log t}\right]\right\|+\frac{\log t}{t^{3}}\left\|b_{j}-b_{i}+\frac{c_{j}-c_{i}}{\log t}\right\|\right\} .
$$


Estimates for $\left(1+\frac{G_{i j}}{A_{i j}}\right)^{-\frac{3}{2}}-1$ can be gotten as follows. The Lagrange mean value theorem implies that

$$
\left(1+\frac{G_{i j}}{A_{i j}^{2}}\right)^{-\frac{3}{2}}-1=-\frac{3}{2} \frac{G_{i j}}{A_{i j}^{2}}\left(1+\xi_{i j}\right)^{-\frac{5}{2}},
$$

for some real number $\xi_{i j}$ satisfying $0<\left|\xi_{i j}\right|<\left|\frac{G_{i j}}{A_{i j}^{2}}\right|$. Recall that by Lemma 2, given any $\rho<1$ and any $K_{0}>4 \hat{b} / \underline{A}$, there exists $t_{0}$ independent of $i$ and $j$, such that for $t \geq t_{0}$ we have

$$
0<\left|\xi_{i j}\right|<\left|\frac{G_{i j}}{A_{i j}^{2}}\right| \leq K_{0} \frac{\log t}{t} \leq \rho .
$$

Therefore,

$$
\left|\left(1+\frac{G_{i j}}{A_{i j}^{2}}\right)^{-\frac{3}{2}}-1\right|=\left|-\frac{3}{2} \frac{G_{i j}}{A_{i j}^{2}}\left(1+\xi_{i j}\right)^{-\frac{5}{2}}\right| \leq \frac{3}{2}(1-\rho)^{-\frac{5}{2}} K_{0} \frac{\log t}{t} \leq \frac{3}{2} \frac{\rho}{(1-\rho)^{\frac{5}{2}}} .
$$

We now collect the estimates on each summand in the representation of $P 1_{i}(t)$ in (5.2.13). Invoking (5.2.15) and the triangle inequality, we have

$$
\begin{array}{r}
\left\|P 1_{i}(t)\right\| \leq \frac{1}{t^{2}} \sum_{j \neq i} \frac{m_{j}}{\underline{A}^{3}}\left\{\frac{3}{2}(1-\rho)^{-\frac{5}{2}} K_{0} \frac{\log t}{t}\left(2 \hat{a}+\frac{\log t}{t}\left[2 \hat{b}+\frac{2 \hat{c}}{\log t}\right]\right)+\frac{\log t}{t^{3}}\left(2 \hat{b}+\frac{2 \hat{c}}{\log t}\right)\right\} \\
\left\|P 1_{i}(t)\right\| \leq \frac{3 M}{\underline{A}^{3}} \frac{\log t}{t^{3}}\left\{(1-\rho)^{-\frac{5}{2}} K_{0}\left(\hat{a}+\frac{\log t}{t}\left[\hat{b}+\frac{\hat{c}}{\log t}\right]\right)+\frac{2}{3 t^{2}}\left(\hat{b}+\frac{\hat{c}}{\log t}\right)\right\} .
\end{array}
$$

where $M=\sum_{j} m_{j}$ and $\hat{a}=\max _{k}\left\|a_{k}\right\|$. Combining the above with arguments analogous to those of Lemma 2 , we obtain

Lemma 32. Assume (5.1.6) holds and let $b$ be given by (5.1.5). Then for any $\rho, 0<\rho<1$, any $K_{0}>4 \hat{b} / \underline{A}$, and any

there exists $t_{1} \geq t_{0}$ such that for $t \geq t_{1}$

$$
K_{1}>\frac{3 M \hat{a}}{\underline{A}^{3}}(1-\rho)^{-\frac{5}{2}} K_{0}
$$

$$
\left\|P 1_{i}(t)\right\| \leq K_{1} \frac{\log t}{t^{3}}
$$

for all $i$.

In the sequel we will need the double integral

$$
L_{i}(t):=\int_{\infty}^{t} \int_{\infty}^{s} P 1_{i}(u) d u d s
$$

To bound $L_{i}$, we will estimate the integral

LEMma 33. The double integral

$$
U(t):=\int_{t}^{\infty} \int_{s}^{\infty} u^{-r} \log u d u d s=\frac{\log t}{(r-1)(r-2) t^{r-2}}+\frac{2 r-3}{(r-1)^{2}(r-2)^{2} t^{r-2}} \leq \frac{\log t}{(r-2)^{2} t^{r-2}},
$$

for $t \geq e^{2}$.

Proof.

$$
\int_{t}^{\infty} \int_{s}^{\infty} u^{-r} \log u d u d s=\int_{t}^{\infty} \int_{s}^{\infty} \frac{d}{d u}\left(-\frac{u^{-r+1}}{r-1}\right) \log u d u d s
$$

for $r>2$. First we calculate the inner integral by parts

$$
V(s):=\int_{s}^{\infty} \frac{d}{d u}\left(-\frac{u^{-r+1}}{r-1}\right) \log u d u=\frac{s^{-r+1}}{r-1} \log s+\int_{s}^{\infty} \frac{u^{-r}}{r-1} d u=\frac{s^{-r+1}}{r-1} \log s+\frac{s^{-r+1}}{(r-1)^{2}} .
$$


Then

$$
\begin{gathered}
U(t)=\int_{t}^{\infty} V(s) d s=\int_{t}^{\infty}\left[\frac{s^{-r+1}}{r-1} \log s+\frac{s^{-r+1}}{(r-1)^{2} \cdot}\right] d s \\
=\frac{t^{-r+2}}{(r-1)(r-2)} \log t+\frac{t^{-r+2}}{(r-1)(r-2)^{2}}+\frac{t^{-r+2}}{(r-1)^{2}(r-2)} \\
=\frac{t^{-r+2}}{(r-1)(r-2)}\left(\log t+\frac{1}{(r-2)}+\frac{1}{(r-1)}\right)=\frac{t^{-r+2}}{(r-1)(r-2)}\left(\log t+\frac{2 r-3}{(r-1)(r-2)}\right) .
\end{gathered}
$$

We can bound this

$$
U(t) \leq \frac{t^{-r+2} \log t}{(r-2)^{2}}
$$

provided

$$
\frac{1}{(r-1)(r-2)}\left(\log t+\frac{2 r-3}{(r-1)(r-2)}\right) \leq \frac{\log t}{(r-2)^{2}} .
$$

We can determine the restriction imposed on $t$ by cross-multiplying

$$
(r-2) \log t+\frac{2 r-3}{r-1} \leq(r-1) \log t
$$

or

$$
\frac{2 r-3}{r-1} \leq \log t
$$

The left-hand side is less than 2 , so the inequality certainly holds for all $t \geq e^{2}$.

By the Lemma above on the double integral of $\log u / u^{r}$, we know that

$$
\left\|L_{i}(t)\right\|=\left\|\int_{\infty}^{t} \int_{\infty}^{s} P 1_{i}(u) d u d s\right\| \leq \int_{\infty}^{t} \int_{\infty}^{s}\left\|P 1_{i}(u)\right\| d u d s \leq K_{1} \int_{\infty}^{t} \int_{\infty}^{s} \frac{\log u}{u^{3}} d u d s \leq K_{1} \frac{\log t}{t},
$$

for $t \geq \max \left\{e^{2}, t_{1}\right\}$. Note that the integral $L_{i}(t) \rightarrow 0$, as $t \rightarrow \infty$.

5.2.2. Estimating $P 2$. Much of the calculation leading to a bound for $P 2_{i}$ is directly analogous to that above for $P 1_{i}$. We start with

$$
\left\|q_{i}-q_{j}\right\|^{2}=\left\|f_{j}-f_{i}+\delta_{j}-\delta_{i}\right\|^{2}=\left\|f_{j}-f_{i}\right\|^{2}+2\left(f_{j}-f_{i}\right)^{\dagger}\left(\delta_{j}-\delta_{i}\right)+\left\|\delta_{j}-\delta_{i}\right\|^{2}=F_{i j}^{2}\left(1+\frac{E_{i j}}{F_{i j}^{2}}\right),
$$

where

$$
E_{i j}(\delta):=2\left(f_{j}-f_{i}\right)^{\dagger}\left(\delta_{j}-\delta_{i}\right)+\left\|\delta_{j}-\delta_{i}\right\|^{2} .
$$

The same calculation that led to $(5.2 .12)$ brings us to a useful expression for $P 2_{i}$

$$
\begin{gathered}
P 2_{i}=\sum_{j \neq i} m_{j}\left(\frac{f_{j}-f_{i}+\delta_{j}-\delta_{i}}{F_{i j}^{3}\left(1+E_{i j} / F_{i j}^{2}\right)^{3 / 2}}-\frac{f_{j}-f_{i}}{F_{i j}^{3}}\right) . \\
=\sum_{j \neq i} \frac{m_{j}}{F_{i j}^{3}}\left\{\left(1+E_{i j} / F_{i j}^{2}\right)^{-3 / 2}\left(f_{j}-f_{i}+\delta_{j}-\delta_{i}\right)-\left(f_{j}-f_{i}\right)\right\} \\
=\sum_{j \neq i} \frac{m_{j}}{F_{i j}^{3}}\left\{\left(\left[1+\frac{E_{i j}}{F_{i j}^{2}}\right]^{-3 / 2}-1\right)\left[f_{j}-f_{i}+\delta_{j}-\delta_{i}\right]+\delta_{j}-\delta_{i}\right\} .
\end{gathered}
$$

We seek a bound for

$$
\begin{aligned}
\left\|P 2_{i}\right\| & \leq \sum_{j \neq i} \frac{m_{j}}{F_{i j}^{2}}\left\{\left|\left[1+\frac{E_{i j}}{F_{i j}^{2}}\right]^{-3 / 2}-1\right| \frac{\left\|f_{j}-f_{i}\right\|+\left\|\delta_{j}-\delta_{i}\right\|}{F_{i j}}+\frac{\left\|\delta_{j}-\delta_{i}\right\|}{F_{i j}}\right\} \\
& \left.=\sum_{j \neq i} \frac{m_{j}}{F_{i j}^{2}}\left\{|| 1+\frac{E_{i j}}{F_{i j}^{2}}\right]^{-3 / 2}-1 \mid\left(1+\frac{\left\|\delta_{j}-\delta_{i}\right\|}{F_{i j}}\right)+\frac{\left\|\delta_{j}-\delta_{i}\right\|}{F_{i j}}\right\} .
\end{aligned}
$$


We now prepare estimates for the individual terms.

We already know from (5.2.15) that

$$
\left(1+\frac{G_{i j}}{A_{i j}^{2}}\right)^{-3 / 2} \leq 1+\frac{3 K_{0}}{2(1-\rho)^{\frac{5}{2}}} \frac{\log t}{t}
$$

so

$$
\frac{1}{F_{i j}^{3}} \leq \frac{1}{A^{3} t^{3}}\left(1+\frac{3 K_{0}}{2(1-\rho)^{\frac{5}{2}}} \frac{\log t}{t}\right) .
$$

Similarly, by the Mean Value Theorem

$$
\left(1+\frac{G_{i j}}{A_{i j}^{2}}\right)^{-1} \leq 1+\frac{K_{0}}{(1-\rho)^{2}} \frac{\log t}{t} \leq 1+\frac{\rho}{(1-\rho)^{2}}
$$

so that

$$
\frac{1}{F_{i j}^{2}} \leq \frac{1}{\underline{A}^{2} t^{2}}\left(1+\frac{K_{0}}{(1-\rho)^{2}} \frac{\log t}{t}\right) \leq \frac{1}{\underline{A}^{2} t^{2}}\left(1+\frac{\rho}{(1-\rho)^{2}}\right)
$$

and

$$
\frac{1}{F_{i j}} \leq \frac{1}{\underline{A} t} \sqrt{1+\frac{K_{0}}{(1-\rho)^{2}} \frac{\log t}{t}} \leq \frac{1}{\underline{A} t} \sqrt{1+\frac{\rho}{(1-\rho)^{2}}}
$$

Next we want an estimate for

$$
\begin{aligned}
E_{i j} & =2\left[\left(a_{j}-a_{i}\right) t+\left(b_{j}-b_{i}\right) \log t+\left(c_{j}-c_{i}\right)\right]^{\dagger}\left(\delta_{j}(t)-\delta_{i}(t)\right)+\left\|\delta_{j}(t)-\delta_{i}(t)\right\|^{2} \\
& =2 t\left[a_{j}-a_{i}+\left(b_{j}-b_{i}\right) \frac{\log t}{t}+\frac{c_{j}-c_{i}}{t}\right]^{\dagger}\left(\delta_{j}(t)-\delta_{i}(t)\right)+\left\|\delta_{j}-\delta_{i}\right\|^{2} .
\end{aligned}
$$

By the Schwarz inequality

$$
\left|\left[a_{j}-a_{i}+\left(b_{j}-b_{i}\right) \frac{\log t}{t}+\frac{c_{j}-c_{i}}{t}\right]^{\dagger}\left(\delta_{j}(t)-\delta_{i}(t)\right)\right| \leq\left\|a_{j}-a_{i}+\left(b_{j}-b_{i}\right) \frac{\log t}{t}+\frac{c_{j}-c_{i}}{t}\right\|\left\|\delta_{j}(t)-\delta_{i}(t)\right\|,
$$

so that

$$
\frac{\left|E_{i j}\right|}{F_{i j}^{2}} \leq \frac{2\left\|a_{j}-a_{i}+\left(b_{j}-b_{i}\right) \frac{\log t}{t}+\frac{c_{j}-c_{i}}{t}\right\|\left\|\delta_{j}-\delta_{i}\right\|+\frac{1}{t}\left\|\delta_{j}-\delta_{i}\right\|^{2}}{A_{i j}^{2}\left(1-\frac{\left|G_{i j}\right|}{A_{i j}^{2}}\right) t} .
$$

Assuming $\delta_{i}=o(1)$ as $t \rightarrow \infty$ (as will be shown in the main theorem), then for any $\epsilon \in(0,1)$ there is a time $t_{2} \geq t_{1}$ such that each $\left\|\delta_{i}(t)\right\| \leq \epsilon$ for $t \geq t_{2}$. So we have by the triangle inequality,

$$
\frac{\left|E_{i j}\right|}{F_{i j}^{2}} \leq \frac{2\left(2 \hat{a}+2 \hat{b} \frac{\log t}{t}+\frac{2 \hat{c}}{t}\right) 2 \epsilon+\frac{4 \epsilon^{2}}{t}}{\underline{A}^{2}\left(1-\frac{\left|G_{i j}\right|}{A_{i j}^{2}}\right) t} \leq \frac{4 \epsilon}{\underline{A}^{2} t} \frac{\hat{a}+\hat{b} \frac{\log t}{t}+\frac{\hat{c}}{t}+\frac{\epsilon}{t}}{1-K_{0} \frac{\log t}{t}} \leq \frac{4}{\underline{A}^{2} t} \frac{\hat{a}+\hat{b} \frac{\log t}{t}+\frac{\hat{c}+1}{t}}{1-K_{0} \frac{\log t}{t}} .
$$

Given any $K_{2} \geq \frac{4 \hat{a}}{A^{2}}$ and any $\rho_{1}, 0<\rho_{1}<1$, there is a time $t_{3}=\max \left\{t_{2}, K_{2} / \rho\right\}$ such that for $t \geq t_{3}$, we have

$$
\frac{\left|E_{i j}\right|}{F_{i j}^{2}} \leq \frac{K_{2}}{t} \leq \rho_{1}<1
$$

As before, the Lagrange mean value theorem implies that for some $\xi_{i j}, 0<\left|\xi_{i j}\right|<\frac{\left|E_{i j}\right|}{F_{i j}^{2}}$

so we can bound

$$
\left(1+\frac{E_{i j}}{F_{i j}^{2}}\right)^{-\frac{3}{2}}-1=-\frac{3}{2} \frac{E_{i j}}{F_{i j}^{2}}\left(1+\xi_{i j}\right)^{-\frac{5}{2}}
$$

$$
\left|\left(1+\frac{E_{i j}}{F_{i j}^{2}}\right)^{-\frac{3}{2}}-1\right| \leq \frac{3}{2}\left|\frac{E_{i j}}{F_{i j}^{2}}\left(1+\xi_{i j}\right)^{-\frac{5}{2}}\right| \leq \frac{3}{2}(1-\rho)^{-\frac{5}{2}} \frac{K_{2}}{t} \leq \frac{3}{2} \frac{\rho}{(1-\rho)^{\frac{5}{2}}} .
$$


Note that this can be made as small as desired by making $\rho$ sufficiently small.

Now we put the pieces together to develop a bound for $P 2_{i}$ from (5.2.23)

$$
\begin{aligned}
\left\|P 2_{i}\right\| & \leq \frac{M}{\underline{A}^{2} t^{2}}\left(1+\frac{K_{0}}{(1-\rho)^{2}} \frac{\log t}{t}\right)\left\{\frac{3}{2}(1-\rho)^{-\frac{5}{2}} \frac{K_{2}}{t}\left(1+\frac{2 \epsilon}{\underline{A t}} \sqrt{1+\frac{\rho}{(1-\rho)^{2}}}\right)+\frac{2 \epsilon}{\underline{A t} \sqrt{1+\frac{\rho}{(1-\rho)^{2}}}}\right\} \\
\leq & \frac{M}{\underline{A}^{2} t^{3}}\left(1+\frac{K_{0}}{(1-\rho)^{2}} \frac{\log t}{t}\right)\left\{\frac{3}{2} \frac{K_{2}}{(1-\rho)^{\frac{5}{2}}}\left(1+\frac{2 \epsilon}{\underline{A} t} \sqrt{1+\frac{\rho}{(1-\rho)^{2}}}\right)+\frac{2 \epsilon}{\underline{A}} \sqrt{1+\frac{\rho}{(1-\rho)^{2}}}\right\} .
\end{aligned}
$$

And for any

$$
K_{3}>\frac{3}{2} \frac{M K_{2}}{\underline{A}^{2}(1-\rho)^{\frac{5}{2}}},
$$

there is a time $t_{4} \geq t_{3}$ such that $\left\|P 2_{i}\right\| \leq K_{3} / t^{3}$ for $t \geq t_{4}$.

5.2.3. Estimating $P 2(\delta)-P 2(\eta)$ in terms of $\delta-\eta$. For the induction in the next Section, we will also need to bound $P 2_{i}(\delta, t)-P 2_{i}(\eta, t)$ for small $\delta-\eta$. To simplify the formulas, let us define the shorthand

$$
J_{i j}(\delta):=\left[1+\frac{E_{i j}(\delta)}{F_{i j}^{2}}\right]^{-3 / 2} .
$$

Then

$$
\begin{gathered}
P 2_{i}(\delta, t)-P 2_{i}(\eta, t) \\
=\sum_{j \neq i} \frac{m_{j}}{F_{i j}^{3}}\left\{\left(J_{i j}(\delta)-1\right)\left[f_{j}-f_{i}+\delta_{j}-\delta_{i}\right]-\left(J_{i j}(\eta)-1\right)\left[f_{j}-f_{i}+\eta_{j}-\eta_{i}\right]+\delta_{j}-\delta_{i}-\eta_{j}+\eta_{i}\right\}
\end{gathered}
$$

which simplifies due to cancellation:

$$
P 2_{i}(\delta, t)-P 2_{i}(\eta, t)=\sum_{j \neq i} \frac{m_{j}}{F_{i j}^{3}}\left\{J_{i j}(\delta)\left[f_{j}-f_{i}+\delta_{j}-\delta_{i}\right]-J_{i j}(\eta)\left[f_{j}-f_{i}+\eta_{j}-\eta_{i}\right]\right\} .
$$

Suppose that for all $i,\left\|\delta_{i}-\eta_{i}\right\| \leq w$. Because of the bound (5.2.27), we have

$$
\left|J_{i j}(\delta)\right| \leq\left[1-\frac{K_{2}}{t}\right]^{-3 / 2}
$$

Then we may express the quantity within the brackets of (5.2.29)

$$
\begin{gathered}
J_{i j}(\delta)\left[f_{j}-f_{i}+\delta_{j}-\delta_{i}\right]-J_{i j}(\eta)\left[f_{j}-f_{i}+\eta_{j}-\eta_{i}\right] \\
=\left(J_{i j}(\delta)-J_{i j}(\eta)\right)\left[f_{j}-f_{i}\right]+J_{i j}(\delta)\left[\delta_{j}-\delta_{i}\right]-J_{i j}(\eta)\left[\eta_{j}-\eta_{i}\right] .
\end{gathered}
$$

On adding and subtracting $J_{i j}(\eta)\left(\delta_{j}-\delta_{i}\right)$, we can rewrite it as

$$
\begin{gathered}
\left(J_{i j}(\delta)-J_{i j}(\eta)\right)\left[f_{j}-f_{i}\right]+J_{i j}(\delta)\left[\delta_{j}-\delta_{i}\right]-J_{i j}(\eta)\left(\delta_{j}-\delta_{i}\right)+J_{i j}(\eta)\left(\delta_{j}-\delta_{i}\right)-J_{i} j(\eta)\left[\eta_{j}-\eta_{i}\right] \\
=\left(J_{i j}(\delta)-J_{i j}(\eta)\right)\left[f_{j}-f_{i}\right]+\left[J_{i j}(\delta)-J_{i j}(\eta)\right]\left(\delta_{j}-\delta_{i}\right)+J_{i j}(\eta)\left(\left[\delta_{j}-\delta_{i}\right]-\left[\eta_{j}-\eta_{i}\right]\right) \\
=\left(J_{i j}(\delta)-J_{i j}(\eta)\right)\left[f_{j}-f_{i}+\delta_{j}-\delta_{i}\right]+J_{i j}(\eta)\left(\left[\delta_{j}-\eta_{j}\right]-\left[\delta_{i}-\eta_{i}\right]\right) .
\end{gathered}
$$

In summary,

$$
P 2_{i}(\delta, t)-P 2_{i}(\eta, t)=\sum_{j \neq i} \frac{m_{j}}{F_{i j}^{3}}\left\{\left(J_{i j}(\delta)-J_{i j}(\eta)\right)\left[f_{j}-f_{i}+\delta_{j}-\delta_{i}\right]+J_{i j}(\eta)\left(\left[\delta_{j}-\eta_{j}\right]-\left[\delta_{i}-\eta_{i}\right]\right)\right\}
$$

so we have

$$
\left\|P 2_{i}(\delta, t)-P 2_{i}(\eta, t)\right\| \leq \sum_{j \neq i} \frac{m_{j}}{F_{i j}^{3}}\left\{\left|J_{i j}(\delta)-J_{i j}(\eta)\right|\left(F_{i j}+\left\|\delta_{j}-\delta_{i}\right\|\right)+\left|J_{i j}(\eta)\right|\left(\left\|\delta_{j}-\eta_{j}\right\|+\left\|\delta_{i}-\eta_{i}\right\|\right)\right\}
$$

$$
\left\|P 2_{i}(\delta, t)-P 2_{i}(\eta, t)\right\| \leq \sum_{j \neq i} \frac{m_{j}}{F_{i j}^{2}}\left\{\left|J_{i j}(\delta)-J_{i j}(\eta)\right|\left(1+\frac{2 \epsilon}{F_{i j}}\right)+\frac{1}{F_{i j}}\left(1-\frac{K_{2}}{t}\right)^{-3 / 2} 2 w\right\}
$$


We start with an estimate of $J_{i j}(\delta)-J_{i j}(\eta)$ for small $\delta-\eta$. By the mean value theorem, there is a number $\xi_{i j}$ between $\frac{E_{i j}(\delta)}{F_{i j}^{2}}$ and $\frac{E_{i j}(\eta)}{F_{i j}^{2}}$ such that

$$
J_{i j}(\delta)-J_{i j}(\eta)=\left(1+\frac{E_{i j}(\delta)}{F_{i j}^{2}}\right)^{-3 / 2}-\left(1+\frac{E_{i j}(\eta)}{F_{i j}^{2}}\right)^{-3 / 2}=-\frac{3}{2} \frac{E_{i j}(\delta)-E_{i j}(\eta)}{F_{i j}^{2}}\left(1+\xi_{i j}\right)^{-5 / 2} .
$$

Since we have

$$
\frac{\left|E_{i j}\right|}{F_{i j}^{2}} \leq \frac{K_{2}}{t}
$$

we know that

$$
\left|J_{i j}(\delta)-J_{i j}(\eta)\right| \leq \frac{3}{2} \frac{\left|E_{i j}(\delta)-E_{i j}(\eta)\right|}{F_{i j}^{2}}\left(1-\frac{K_{2}}{t}\right)^{-5 / 2} .
$$

Next we seek a bound on $\left|E_{i j}(\delta)-E_{i j}(\eta)\right|$ in terms of $\delta$ and $\eta$.

$$
E_{i j}(\delta)-E_{i j}(\eta)=2\left(f_{j}-f_{i}\right)^{\dagger}\left(\delta_{j}-\delta_{i}-\eta_{j}+\eta_{i}\right)+\left\|\delta_{j}-\delta_{i}\right\|^{2}-\left\|\eta_{j}-\eta_{i}\right\|^{2} .
$$

The first term of (5.2.34) is bounded by $2 F_{i j}\left(\left\|\delta_{j}-\eta_{j}\right\|+\left\|\delta_{i}-\eta_{i}\right\|\right) \leq 4 F_{i j} w$. The difference between the squares in (5.2.34) factors

$$
\left\|\delta_{j}-\delta_{i}\right\|^{2}-\left\|\eta_{j}-\eta_{i}\right\|^{2}=\left(\delta_{j}-\delta_{i}+\eta_{j}-\eta_{i}\right)^{\dagger}\left(\delta_{j}-\delta_{i}-\eta_{j}+\eta_{i}\right),
$$

which is bounded by

$$
\left(\left\|\delta_{j}-\delta_{i}\right\|+\left\|\eta_{j}-\eta_{i}\right\|\right)\left(\left\|\delta_{j}-\eta_{j}\right\|+\left\|\delta_{i}-\eta_{i}\right\|\right) \leq(2 \epsilon+2 \epsilon) 2 w=8 \epsilon w .
$$

So we have

$$
\left|J_{i j}(\delta)-J_{i j}(\eta)\right| \leq \frac{3}{2} \frac{4 F_{i j} w+8 \epsilon w}{F_{i j}^{2}}\left(1-\frac{K_{2}}{t}\right)^{-5 / 2}=\left[\frac{6 w}{F_{i j}}+\frac{12 \epsilon w}{F_{i j}^{2}}\right]\left(1-\frac{K_{2}}{t}\right)^{-5 / 2} .
$$

In summary, we can now bound (5.2.33)

$$
\begin{gathered}
\left\|P 2_{i}(\delta, t)-P 2_{i}(\eta, t)\right\| \leq \sum_{j \neq i} \frac{m_{j}}{F_{i j}^{2}}\left\{\left(\left[\frac{6 w}{F_{i j}}+\frac{12 \epsilon w}{F_{i j}^{2}}\right]\left(1-\frac{K_{2}}{t}\right)^{-5 / 2}\right)\left(1+\frac{2 \epsilon}{F_{i j}}\right)+\frac{2 w}{F_{i j}}\left[1-\frac{K_{2}}{t}\right]^{-3 / 2}\right\} \\
\left\|P 2_{i}(\delta, t)-P 2_{i}(\eta, t)\right\| \leq 2 w \sum_{j \neq i} \frac{m_{j}}{F_{i j}^{3}}\left\{\left(\left[3+\frac{6 \epsilon}{F_{i j}}\right]\left(1-\frac{K_{2}}{t}\right)^{-5 / 2}\right)\left(1+\frac{2 \epsilon}{F_{i j}}\right)+\left[1-\frac{K_{2}}{t}\right]^{-3 / 2}\right\} .
\end{gathered}
$$

Because of the bound (5.2.24), we conclude

LEMma 34. Under the same conditions as Lemma 32, and for any $K_{4}>\frac{8 M}{A^{3}}$, there is a time $t_{4} \geq t_{3}$ independent of $i$ such that for $t \geq t_{4}$,

$$
\left\|P 2_{i}(\delta, t)-P 2_{i}(\eta, t)\right\| \leq K_{4} w / t^{3},
$$

where $w$ is a bound for all $\left\|\delta_{i}-\eta_{i}\right\|$.

\subsection{The integral equation}

In this Section we return to the integral equation (5.1.10) at the end of Section 5.1. We propose an iterative solution

$$
\delta_{i}[n+1]=\int_{t}^{\infty} \int_{s}^{\infty}\left(P 1_{i}(u)+P 2_{i}(\delta[n](u), u)\right) d u d s,
$$

starting with each $\delta_{i}[0]=$ the zero vector. Having developed estimates for $P 1_{i}$ and $P 2_{i}$, we can show that the sequence $\left\{\delta_{i}[n]\right\}$ converges to a function $\delta_{i}$ with the requisite properties. With the definition (5.2.18) we can rewrite (5.3.1) as

$$
\delta_{i}[n+1]=L_{i}(t)+\int_{t}^{\infty} \int_{s}^{\infty} P 2_{i}(\delta[n], u) d u d s .
$$

We give induction arguments to show that for all $n$ and for all $i,\left\|\delta_{i}[n]\right\|=O(\log t / t)$ and $\left\|\delta_{i}[n+1]-\delta_{i}[n]\right\|=$ $O\left(\log t / t^{n+1}\right)$ as $t \rightarrow \infty$. 
TheOREM 35. Under the conditions of Lemma 34, the sequence $\left\{\delta_{i}[n]\right\}$ converges to a function $\delta_{i}$, which satisfies the integral equation (5.1.10), as well as the differential equation (5.1.7).

Proof. Since each $\delta_{i}[0](t)=\overrightarrow{0}$, so is every $P 2_{i}=\overrightarrow{0}$, and we have

$$
\delta_{i}[1]=L_{i}(t)
$$

and as noted above (5.2.20) $\left\|L_{i}\right\| \leq K_{1} \frac{\log t}{t}$, for $t \geq t_{4}$, so

$$
\left\|\delta_{i}[1]-\delta_{i}[0]\right\|=\left\|\delta_{i}[1]\right\|=\left\|L_{i}\right\| \leq K_{1} \frac{\log t}{t} .
$$

In general, starting with $\delta_{i}[n]$ and making the induction hypothesis that for $t \geq t_{4}$

$$
\left\|\delta_{i}[n]-\delta_{i}[n-1]\right\| \leq C_{n} \frac{\log t}{t^{n}}, \quad i=1, \ldots, N,
$$

where $C_{1}=K_{1}$ is defined in Lemma 32 and

$$
C_{n+1}=\frac{K_{4} C_{n}}{(n+1)^{2}} ; \text { i.e., } C_{n}=\frac{K_{4}^{n-1}}{(n !)^{2}} K_{1},
$$

We will show that the corresponding statement holds for $n+1$.

First, from the formula

$$
\delta[n]=\delta[1]+(\delta[2]-\delta[1])+\cdots+(\delta[n]-\delta[n-1])
$$

and using the induction hypothesis, we can bound

$$
\begin{aligned}
\left\|\delta_{i}[n]\right\| & \leq\left\|\delta_{i}[1]\right\|+\left\|\delta_{i}[2]-\delta_{i}[1]\right\|+\cdots+\left\|\delta_{i}[n]-\delta_{i}[n-1]\right\| \\
& \leq C_{1} \frac{\log t}{t}+C_{2} \frac{\log t}{t^{2}}+\cdots+C_{n} \frac{\log t}{t^{n}} \\
& =K_{1} \frac{\log t}{t}+\frac{K_{4}}{4} K_{1} \frac{\log t}{t^{2}}+\cdots+\frac{K_{4}^{n-1}}{(n !)^{2}} K_{1} \frac{\log t}{t^{n}} \\
& =K_{1} \frac{\log t}{t}\left(1+\frac{K_{4}}{4 t}+\cdots+\frac{K_{4}^{n-1}}{(n !)^{2} t^{n-1}}\right) \\
& \leq K_{1} \frac{\log t}{t} \sum_{k=1}^{\infty} \frac{1}{(k !)^{2}},
\end{aligned}
$$

for $t \geq K_{4}$. According to the tables in Gradshteyn \& Ryzhik [45] Section 0.246, the series sums to the number $I_{0}(2)-1 \sim 1.2795853<1.28$. The function $\frac{\log t}{t}$ is monotone on the interval $(e, \infty)$, decreasing from $1 / e$ at $t=e$ and approaching 0 as $t \rightarrow \infty$. For any $\epsilon<1$, there is a time $t_{5}=\max \left\{4, t_{4}, K_{4}\right\}$, such that, for all $n$

$$
t \geq t_{5} \Longrightarrow\left\|\delta_{i}[n]\right\| \leq 1.28 K_{1} \frac{\log t}{t} \leq \epsilon<1 .
$$

This bound is independent of $n$, so the sequence $\left\{\delta_{i}[n]\right\}$ is bounded for $t \geq t_{5}$, and the bound can in fact be made as small as desired by choosing sufficiently large $t_{5}$.

The goal of the induction is to show that the sequence is Cauchy; note that

$$
\delta_{i}[n+1]-\delta_{i}[n]=\int_{\infty}^{t} \int_{\infty}^{s}\left(P 2_{i}(\delta[n])-P 2_{i}(\delta[n-1])\right) .
$$

We have shown in Section 5.2 that if $w$ is a bound on all the $\left\|\delta_{i}[n]-\delta_{i}[n-1]\right\|, i=1, \ldots, N$, then

According to (5.3.2), we can choose

$$
\left\|P 2_{i}(\delta[n])-P 2_{i}(\delta[n-1])\right\| \leq K_{4} w / t^{3}
$$

Then we have

$$
w=C_{n} \frac{\log t}{t^{n}}
$$

$$
\left\|\delta_{i}[n+1]-\delta_{i}[n]\right\| \leq K_{4} C_{n} \int_{\infty}^{t} \int_{\infty}^{s} \frac{\log u}{u^{n+3}} d u d s \leq \frac{K_{4} C_{n}}{(n+1)^{2}} \frac{\log t}{t^{n+1}}=C_{n+1} \frac{\log t}{t^{n+1}},
$$


which completes the induction.

For convergence considerations, note that the series

$$
\sum_{n=1}^{\infty} \frac{C_{n}}{t^{n}}
$$

converges for all $t>0$ by the ratio test, since the ratio of successive terms

$$
\frac{C_{n+1}}{C_{n} t}=\frac{K_{4}}{(n+1)^{2} t} \rightarrow 0
$$

as $n \rightarrow \infty$, uniformly for $t \geq t_{5}>0$. Thus the limit of the sum (5.3.3) serves to define the solution

$$
\delta_{i}=\lim _{n \rightarrow \infty} \delta_{i}[n]=\delta_{i}[1]+\sum_{n=2}^{\infty}\left(\delta_{i}[n]-\delta_{i}[n-1]\right)
$$

and each

$$
\left\|\delta_{i}\right\| \leq \epsilon<1
$$

To obtain the rate of convergence of the series, we have the error after the $n^{\text {th }}$ term:

$$
\begin{gathered}
\left\|\delta_{i}(t)-\delta_{i}[n](t)\right\|=\left\|\sum_{j=n+1}^{\infty}\left(\delta_{i}[j](t)-\delta_{i}[j-1](t)\right)\right\| \leq \sum_{j=n+1}^{\infty} C_{j} \frac{\log t}{t^{j}} \\
=\sum_{j=n+1}^{\infty} \frac{K_{4}^{j-1}}{(j !)^{2}} K_{1} \frac{\log t}{t^{j}}=K_{1} \frac{\log t}{t} \sum_{j=n+1}^{\infty} \frac{1}{(j !)^{2}} \frac{K_{4}^{j-1}}{t^{j-1}}=\frac{\log t}{t}\left\{\frac{1}{[(n+1) !]^{2}}\left(\frac{K_{4}}{t}\right)^{n}+\sum_{j=n+2}^{\infty} \frac{1}{(j !)^{2}}\left(\frac{K_{4}}{t}\right)^{j-1}\right\} \\
=K_{1} \frac{1}{[(n+1) !]^{2}}\left(\frac{K_{4}}{t}\right)^{n} \frac{\log t}{t}\left\{1+\sum_{j=n+2}^{\infty} \frac{[(n+1) !]^{2}}{(j !)^{2}}\left(\frac{K_{4}}{t}\right)^{j-n-1}\right\}=O\left(\frac{1}{[(n+1) !]^{2}} \frac{\log t}{t}\right) .
\end{gathered}
$$

Notice that for $t \geq t_{5} \geq K_{4}$, we have

$$
\sum_{j=n+2}^{\infty} \frac{[(n+1) !]^{2}}{(j !)^{2}}\left(\frac{K_{4}}{t}\right)^{j-n-1} \leq \sum_{j=n+2}^{\infty} \frac{[(n+1) !]^{2}}{(j !)^{2}}\left(\frac{K_{4}}{t_{5}}\right)^{j-n-1} .
$$

The infinite series $\sum_{j=n+2}^{\infty} \frac{[(n+1) !]^{2}}{(j !)^{2}}\left(\frac{K_{4}}{t_{5}}\right)^{j-n-1}$ converges very fast as the ratio of two successive terms is $\frac{K_{4}}{(j+1)^{2} t_{5}} \leq \frac{1}{(j+1)^{2}}, j \geq n+2$.

We turn to high order asymptotic approximations. By adding and subtracting $\delta_{i}[n](t)$, we can express each

Let

$$
q_{i}=a_{i} t+b_{i} \log t+c_{i}+\delta_{i}[n](t)+\delta_{i}(t)-\delta_{i}[n](t), i=1, \ldots, N .
$$

$$
v_{\text {in }}=a_{i} t+b_{i} \log t+c_{i}+\delta_{i}[n](t)
$$

be the $n^{\text {th }}$ approximation to $q_{i}$. Assume that $a_{i} \neq 0_{3}$; then one of its components $a_{i u} \neq 0$, where $u$ is one of $x, y$, or $z$. Consequently, there exists a continuous 3 -vector $w_{\text {in }}=w_{\text {in }}(t)$ on a semi-infinite interval $\left[t_{5}, \infty\right)$ such that

$$
w_{i n}^{\dagger} v_{i n}=1,\left\|w_{i n}\right\|=O\left(t^{-1}\right) \text {, as } t \rightarrow \infty .
$$

For example, let $u=x$. Then, $w_{i n}^{\dagger}=\left(\left[a_{i x} t+b_{i x} \log t+c_{i x}+\delta_{i x}[n](t)\right]^{-1}, 0,0\right)$ is one of the continuous vectors that satisfies $w_{i n}^{\dagger} v_{i n}=1$. Notice that

$$
\delta_{i}(t)-\delta_{i}[n](t)=\left(\delta_{i}(t)-\delta_{i}[n](t)\right) w_{i n}^{\dagger} v_{i n}=\left(\delta_{i}(t)-\delta_{i}[n](t)\right) w_{i n}^{\dagger}\left(a_{i} t+b_{i} \log t+c_{i}+\delta_{i}[n](t)\right) .
$$

So we can express 


$$
q_{i}=\left\{I+\left(\delta_{i}(t)-\delta_{i}[n](t)\right) w_{i n}^{\dagger}\right\}\left(a_{i} t+b_{i} \log t+c_{i}+\delta_{i}[n](t)\right),
$$

with

$$
\left(\delta_{i}(t)-\delta_{i}[n](t)\right) w_{i n}^{\dagger}=O\left(\frac{K_{4}^{n}}{[(n+1) !]^{2}} \frac{\log t}{t^{n+2}}\right),
$$

uniformly for $t \in\left[t_{5}, \infty\right)$ as $n \rightarrow \infty$, which is (5.0.5). If one of the $a_{i}=0_{3}$, but $b_{i} \neq 0_{3}$, then the resulting asymptotic formula will be

$$
\left(\delta_{i}(t)-\delta_{i}[n](t)\right) w_{i n}^{\dagger}=O\left(\frac{K_{4}^{n}}{[(n+1) !]^{2}} \frac{1}{t^{n+2}}\right),
$$

uniformly for $t \in\left[t_{5}, \infty\right)$ as $n \rightarrow \infty$.

Now recall from Section 5.2 the notation $F_{i j}^{2}=\left\|f_{i}-f_{j}\right\|$. Starting with $q_{i}-q_{j}=f_{i}-f_{j}+\delta_{i}-\delta_{j}$ and substituting from (5.2.21) and (5.2.7), we have

$$
\left\|q_{i}-q_{j}\right\|^{2}=F_{i j}^{2}\left(1+\frac{E_{i j}}{F_{i j}^{2}}\right)=A_{i j}^{2} t^{2}\left(1+\frac{G_{i j}}{A_{i j}^{2}}\right)\left(1+\frac{E_{i j}}{F_{i j}^{2}}\right) .
$$

By virtue of the estimates (5.2.8) and (5.2.27), we know that for $t \geq t_{3}$,

$$
\left|\frac{G_{i j}}{A_{i j}^{2}}\right| \leq \rho<1, \quad\left|\frac{E_{i j}}{F_{i j}^{2}}\right| \leq \rho_{1}<1
$$

so that

$$
\left\|q_{i}-q_{j}\right\|^{2} \geq A_{i j}^{2} t^{2}(1-\rho)\left(1-\rho_{1}\right)
$$

is bounded away from 0 . Thus for all $i \neq j$, for $t \geq t_{5}$, we have $\left\|q_{i}-q_{j}\right\|>0$, and there is no singularity, nor does the system approach collision as $t \rightarrow \infty$. This completes the proof of Theorem 29 .

The compactification of $q$ in Section 4.3 shows that $q$ has a critical direction $x^{*}$ at infinity. Note also that if $x$ is the compactification of $q$, then $x \sim \bar{x}$.

We offer the following interpretation of the parameters, as well as a proof of the special case mentioned in Theorem 29. Each $a_{i}$ is evidently the asymptotic velocity of the $i^{\text {th }}$ particle. The $b_{i}$ are coefficients of the asymptotic acceleration as $\mathrm{t}$ goes to infinity. The $c_{i}$ represent asymptotic translation of an individual particle. Moreover, as seen in the main theorem, $c_{i}$ can be interpreted as the asymptotic limit as $t \rightarrow \infty$ of the position of precisely one particle in our configuration for a certain choice of parameters. In Theorem 29 , we asserted the existence of the special case where one particle tends to a fixed, finite position, and the others escape to infinity as $t \rightarrow \infty$. This is also especially easy to see for $N=3$ : take $a_{1}=0, a_{2} \neq 0$, and $a_{3}=-m_{2} / m_{3} a_{2}$. Then $b_{1}=0$, and since each $\delta_{i} \rightarrow 0$, we have $q_{1} \rightarrow c_{1}$, while $q_{2}$ and $q_{3}$ escape in opposite directions. For $N>3$, suppose $a_{1}=0$, the $a_{2}, \ldots, a_{N-1}$ are linearly independent, but otherwise arbitrary vectors, and choose $a_{N}$ so that

$$
\frac{a_{N}}{\left\|a_{N}\right\|^{3}}=-\frac{1}{m_{N}} \sum_{j=2}^{N-1} \frac{m_{j} a_{j}}{\left\|a_{j}\right\|^{3}}
$$

for example $a_{N}$ could be the unit vector in the direction of the right-hand side of (5.3.12). Then $b_{1}=0$, and we have $q_{1} \rightarrow c_{1}$.

The theorem may be read as the converse of Chazy's result that if solutions exist for all time, then the functions $\delta_{i}$ can be expressed as psi-series; that is, as a series in powers of $1 / t$ and $\log t / t$. Since the functions $\delta_{i}$ have been shown here to exist, the solutions $q_{i}$ exist for all time, and we know from Chazy that the $\delta_{i}$ can be expressed as psi-series. See Kozlov and Palamodov [62] for information on psi-series. We offer the conjecture that $\delta_{i}$ has an even simpler form:

$$
\delta \sim \sum_{n=1}^{\infty}\left[\frac{g_{n}}{t^{n}}+\log t \frac{h_{n}}{t^{n}}\right]
$$

where each $g_{n}$ and $h_{n}$ is a $3 N$-vector. 
Note that by the construction of this Chapter, the initial time $t_{5}$ could be quite large, so the initial conditions

$$
\begin{aligned}
& q_{i}\left(t_{5}\right)=a_{i} t_{5}+b_{i} \log t_{5}+c_{i}+\delta_{i}\left(t_{5}\right) \\
& \dot{q}_{i}\left(t_{5}\right)=a_{i}+b_{i} / t_{5}+\dot{\delta}_{i}\left(t_{5}\right)
\end{aligned}
$$

and the latter is very close to $a_{i}$. As noted above, the asymptotic direction of particle $q_{i}$ exiting the system is proportional to $a_{i}$. This justifies the statement in the Abstract that the asymptotic directions of many configurations exiting the universe depend solely on the initial velocities and not on their initial positions.

\subsection{Comparisons with previous work}

In this Section, we compare our approximations and asymptotic approximations (for an open set of solutions that exist for all forward time) with those of Pollard, Saari, and collaborators. They were able to obtain some asymptotic approximations under the assumption that solutions exist for all forward time. As pointed out in Chapter 1 and proven in the previous Section, our result establishes the existence of an open set of solutions that exist for all forward time, in which all (or all but one) particles escape. As will be seen our asymptotic approximations for these solutions offer much more precision.

For the sake of this comparison with previous work on this subject matter, we make the customary assumption that the coordinate system is fixed at the center of mass, so that

$$
\sum_{k} m_{k} q_{k}=0 \quad \sum_{k} m_{k} \dot{q}_{k}=0 .
$$

This must hold for all values of $t$, so we have the same condition on the components of $q_{k}$ :

$$
\sum_{k} m_{k} a_{k}=0 \quad \sum_{k} m_{k} b_{k}=0 \quad \sum_{k} m_{k} c_{k}=0 \quad \sum_{k} m_{k} \delta_{k}=0 \quad \sum_{k} m_{k} \dot{\delta}_{k}=0 .
$$

The special case of $N=2$ is illustrative; the two-body problem is well known [87] to transform to the Kepler problem for a fictitious particle at $r=q_{2}-q_{1}$, satisfying

$$
\ddot{r}=\left(m_{1}+m_{2}\right) \frac{r}{\|r\|^{3}},
$$

the constant total energy for which is

$$
H=\frac{1}{2}\|\dot{r}\|^{2}-\frac{m_{1}+m_{2}}{\|r\|} .
$$

Since $m_{1} q_{1}+m_{2} q_{2}=0$ and $m_{1} a_{1}+m_{2} a_{2}=0$, we know

$$
r=-\frac{m_{1}+m_{2}}{m_{2}} q_{1} \quad A_{12}=\left\|a_{2}-a_{1}\right\|=\frac{m_{1}+m_{2}}{m_{2}}\left\|a_{1}\right\|,
$$

so that

$$
2 H=\left(\frac{m_{1}+m_{2}}{m_{2}}\right)^{2}\left\|\dot{q}_{1}\right\|^{2}-2 \frac{m_{1}+m_{2}}{\frac{m_{1}+m_{2}}{m_{2}}\left\|q_{1}\right\|}=\left(\frac{m_{1}+m_{2}}{m_{2}}\right)^{2}\left\|\dot{q}_{1}\right\|^{2}-2 \frac{m_{2}}{\left\|q_{1}\right\|},
$$

which is constant and must equal its limit as $t \rightarrow \infty$, namely

$$
2 H=\left(\frac{m_{1}+m_{2}}{m_{2}}\right)^{2}\left\|a_{1}\right\|^{2}=A_{12}^{2} .
$$

Since $H>0, r$ experiences hyperbolic escape, and [87] gives the result that

$$
\|r\| / t \rightarrow \sqrt{2 H} .
$$

Hence we have $\|r\| / t \rightarrow A_{12}$, as expected.

Returning to the general case, let us define the kinetic and potential energy and evaluate them for the expansion

$$
q_{i}=a_{i} t+b_{i} \log t+c_{i}+\delta_{i}(t):
$$




$$
\begin{aligned}
T:=\frac{1}{2} \sum m_{k}\left\|\dot{q}_{k}\right\|^{2} & =\frac{1}{2} \sum m_{k}\left\|a_{k}+\frac{b_{k}}{t}+\dot{\delta}_{k}\right\|^{2}=\frac{1}{2} \sum m_{k}\left\{\left\|a_{k}\right\|^{2}+\frac{2}{t} a_{k}^{\dagger} b_{k}+2 a_{k}^{\dagger} \dot{\delta}_{k}+\left\|\frac{b_{k}}{t}+\dot{\delta}_{k}\right\|^{2}\right\} \\
U:=\sum_{j<k} \frac{m_{j} m_{k}}{\left\|q_{j}-q_{k}\right\|} & =\sum_{j<k} \frac{m_{k}}{\left\|t\left(a_{j}-a_{k}\right)+\log t\left(b_{j}-b_{k}\right)+c_{j}-c_{k}+\delta_{j}-\delta_{k}\right\|} \\
& =\frac{1}{t} \sum_{j<k} \frac{m_{j} m_{k}}{\left\|\left(a_{j}-a_{k}\right)+\frac{\log t}{t}\left(b_{j}-b_{k}\right)+\frac{1}{t}\left(c_{j}-c_{k}+\delta_{j}-\delta_{k}\right)\right\|} .
\end{aligned}
$$

The denominator can be expressed as the square root of

$$
\left\|a_{j}-a_{k}\right\|^{2}+\frac{\log t}{t}\left(a_{j}-a_{k}\right)^{\dagger}\left(b_{j}-b_{k}\right)+\frac{1}{t}\left(a_{j}-a_{k}\right)^{\dagger}\left(c_{j}-c_{k}+\delta_{j}-\delta_{k}\right)+\frac{1}{t^{2}}\left\|c_{j}-c_{k}+\delta_{j}-\delta_{k}\right\|^{2},
$$

which is equal to

$$
\begin{gathered}
\left\|a_{j}-a_{k}\right\|^{2}\left\{1+\frac{\log t}{t}\left(\frac{a_{j}-a_{k}}{\left\|a_{j}-a_{k}\right\|^{2}}\right)^{\dagger}\left(b_{j}-b_{k}\right)\right. \\
\left.+\frac{1}{t}\left(\frac{a_{j}-a_{k}}{\left\|a_{j}-a_{k}\right\|^{2}}\right)^{\dagger}\left(c_{j}-c_{k}+\delta_{j}-\delta_{k}\right)+\frac{1}{t^{2}} \frac{\left\|c_{j}-c_{k}+\delta_{j}-\delta_{k}\right\|^{2}}{\left\|a_{j}-a_{k}\right\|^{2}}\right\} .
\end{gathered}
$$

Let

$$
X_{j k}=\frac{\log t}{t}\left(\frac{a_{j}-a_{k}}{\left\|a_{j}-a_{k}\right\|^{2}}\right)^{\dagger}\left(b_{j}-b_{k}\right)+\frac{1}{t}\left(\frac{a_{j}-a_{k}}{\left\|a_{j}-a_{k}\right\|^{2}}\right)^{\dagger}\left(c_{j}-c_{k}+\delta_{j}-\delta_{k}\right)+\frac{1}{t^{2}} \frac{\left\|c_{j}-c_{k}+\delta_{j}-\delta_{k}\right\|^{2}}{\left\|a_{j}-a_{k}\right\|^{2}},
$$

so that

$$
U=\frac{1}{t} \sum_{j<k} \frac{m_{j} m_{k}}{\left\|a_{j}-a_{k}\right\|}\left(1+X_{j k}\right)^{-1 / 2}
$$

We can bound $X_{j k}$ via the Schwarz inequality, recalling that each $\left\|\delta_{j}\right\| \leq \epsilon$

$$
\left|X_{j k}\right| \leq \frac{\log t}{t} \frac{\left\|b_{j}-b_{k}\right\|}{\left\|a_{j}-a_{k}\right\|}+\frac{1}{t} \frac{\left\|c_{j}-c_{k}\right\|+2 \epsilon}{\left\|a_{j}-a_{k}\right\|}+\frac{1}{t^{2}} \frac{\left\|c_{j}-c_{k}\right\|^{2}+4 \epsilon\left\|c_{j}-c_{k}\right\|+4 \epsilon^{2}}{\left\|a_{j}-a_{k}\right\|^{2}} .
$$

Now for any $\eta>0$, there is a $t_{6} \geq t_{5}$ such that $\left|X_{j k}\right|<\eta$ for all $t \geq t_{6}$. This implies the existence of a convergent series for $\left(1+X_{j k}\right)^{-1 / 2}$ in powers of $X_{j k}$, which is equivalent to a series in powers of $\frac{\log t}{t}$ and $1 / t$, convergent for $t \geq t_{6}$, starting with

$$
1-\frac{\log t}{2 t}\left(\frac{a_{j}-a_{k}}{\left\|a_{j}-a_{k}\right\|^{2}}\right)^{\dagger}\left(b_{j}-b_{k}\right)-\frac{1}{2 t}\left(\frac{a_{j}-a_{k}}{\left\|a_{j}-a_{k}\right\|^{2}}\right)^{\dagger}\left(c_{j}-c_{k}\right) .
$$

So we can express

$$
\begin{array}{r}
U=\frac{1}{t} \sum_{j<k} \frac{m_{j} m_{k}}{\left\|a_{j}-a_{k}\right\|}-\frac{\log t}{2 t^{2}} \sum_{j<k} \frac{m_{j} m_{k}}{\left\|a_{j}-a_{k}\right\|^{3}}\left(a_{j}-a_{k}\right)^{\dagger}\left(b_{j}-b_{k}\right) \\
-\frac{1}{2 t^{2}} \sum_{j<k} \frac{m_{j} m_{k}}{\left\|a_{j}-a_{k}\right\|^{3}}\left(a_{j}-a_{k}\right)^{\dagger}\left(c_{j}-c_{k}\right)+o\left(\frac{1}{t^{2}}\right) .
\end{array}
$$

The constant total energy $h=T-U$ must be equal to its limit as $t \rightarrow \infty$, namely $\frac{1}{2} \sum m_{k}\left\|a_{k}\right\|^{2}>0$. In particular, its $1 / t$ coefficient must vanish; i.e.,

$$
\sum_{k} m_{k} a_{k}^{\dagger} b_{k}=\sum_{j<k} \frac{m_{j} m_{k}}{\left\|a_{j}-a_{k}\right\|}
$$

which holds due to (5.1.5), as follows:

$$
\sum_{k} m_{k} a_{k}^{\dagger} b_{k}=\sum_{k} m_{k} a_{k}^{\dagger} \sum_{j \neq k} \frac{m_{j}\left(a_{k}-a_{j}\right)}{\left\|a_{j}-a_{k}\right\|^{3}}=\sum_{j<k} \frac{m_{j} m_{k}}{\left\|a_{j}-a_{k}\right\|^{3}}\left\{a_{k}^{\dagger}\left(a_{k}-a_{j}\right)+a_{j}^{\dagger}\left(a_{j}-a_{k}\right)\right\}
$$




$$
=\sum_{j<k} \frac{m_{j} m_{k}}{\left\|a_{j}-a_{k}\right\|^{3}}\left\{\left\|a_{k}\right\|^{2}-a_{k}^{\dagger} a_{j}+\left\|a_{j}\right\|^{2}-a_{j}^{\dagger} a_{k}\right\}=\sum_{j<k} \frac{m_{j} m_{k}\left\|a_{j}-a_{k}\right\|^{2}}{\left\|a_{j}-a_{k}\right\|^{3}}=\sum_{j<k} \frac{m_{j} m_{k}}{\left\|a_{j}-a_{k}\right\|} .
$$

Following [86], we define half the moment of inertia

$$
\begin{gathered}
I:=\frac{1}{2} \sum m_{k}\left\|q_{k}\right\|^{2}=\frac{1}{2} \sum m_{k}\left\|a_{k} t+b_{k} \log t+c_{k}+\delta_{k}\right\|^{2} \\
=\frac{1}{2} \sum m_{k}\left\{\left\|a_{k}\right\|^{2} t^{2}+2 t \log t a_{k}^{\dagger} b_{k}+2 t a_{k}^{\dagger}\left(c_{k}+\delta_{k}\right)+\left\|b_{k} \log t+c_{k}+\delta_{k}\right\|^{2}\right\} \\
=h t^{2}+t \log t \sum m_{k} a_{k}^{\dagger} b_{k}+t \sum m_{k} a_{k}^{\dagger} c_{k}+O(\log t) .
\end{gathered}
$$

We are now able to compare our results explicitly with the work of Pollard, Saari, and collaborators. Under the assumption that a solution has no singularity, Pollard in 1967 [86] shows in Theorem 6.1 that if $h>0$ and $U \sim \alpha / t$ for some constant $\alpha>0$, then $I=h t^{2}+\alpha t \log t+o(t \log t)$, as $t \rightarrow \infty$. With the expansion of $I$ given above for our solutions, we see that $\alpha=\sum m_{k} a_{k}^{\dagger} b_{k}=\sum_{j<k} \frac{m_{j} m_{k}}{\left\|a_{j}-a_{k}\right\|}, I$ has the expected form, and we feature the next term in the expansion of $I$. In addition, his Theorem 7.2 says that under the same assumption and conditions, at least $N-1$ of the particles escape to infinity as $t \rightarrow \infty$. It is easy to see for our solutions that every $q_{k}$ escapes, or all escape but one.

In 1970, Pollard and Saari [90] consider the case where $h>0$, but $U$ does not decay as quickly as $\alpha / t$; that is, $t U \rightarrow \infty$, and provide a more general condition:

$$
\int_{t_{0}}^{\infty} \frac{\sqrt{U(s)-\frac{1}{s^{2}} \int_{t_{0}}^{s} u U(u) d u}}{s} d s<\infty
$$

which assures that at least two particles escape.

In 1971, Saari [94] again starts with the assumption that there is no singularity, and he gives the Lemma that if there are two particles, say $q_{1}$ and $q_{2}$ with $\lim \sup \left\|q_{1}-q_{2}\right\|=\infty$, and two particles $q_{i}$ and $q_{j}$ with

$$
0<\liminf \frac{\left\|q_{i}-q_{j}\right\|}{\left\|q_{1}-q_{2}\right\|}<\limsup \frac{\left\|q_{i}-q_{j}\right\|}{\left\|q_{1}-q_{2}\right\|}<\infty
$$

then either

$$
q_{i}=C_{i} t+O(\log t)
$$

or

$$
\left\|q_{i}-q_{j}\right\| \approx t^{2 / 3}
$$

where for two positive functions $f$ and $g, f \approx g$ means that after some time there exist constants $A$ and $B$ such that $A g(t) \leq f(t) \leq B g(t)$. The meaning of the hypothesis is that $q_{1}$ and $q_{2}$ become arbitrarily far apart, and $q_{i}$ and $q_{j}$ do not get closer to each other nor separate at a higher order than $q_{1}$ and $q_{2}$. The conclusion means that either $q_{i}$ grows linearly (or like $\log t$ if $C_{i}=0$ ) or $q_{i}$ and $q_{j}$ separate like $t^{2 / 3}$. Our solutions meet the hypothesis, since all pairs $q_{i}$ and $q_{j}$ separate like $A_{i j} t$. In particular, our solutions adhere to (5.4.3) with at most one $C_{i}=0$.

Saari continues with Theorem 1 (p. 224), which adapted to our restricted setting of particles only, reads as follows:

THEOREM. If solutions to (1.0.1) exist on $(0, \infty)$ and if

$$
\lim \inf _{t \rightarrow \infty} T / U>\frac{1}{2}
$$

then either

$$
q_{j}=a_{j} t+b_{j} \log t+o(\log t)
$$

or

$$
\left\|q_{i}-q_{j}\right\| \approx t^{2 / 3}, i \neq j,
$$

where $a_{j}, b_{j}$ are constant vectors, $\left\|a_{i}-a_{j}\right\| \neq 0$ for $i \neq j$. 
This means in particular that in the absence of behavior Saari calls "oscillatory and pulsating" (which our new solutions do not exhibit), the particles separate into clusters, within which the particle separations are bounded as $t \rightarrow \infty$. The clusters form subsystems wherein the clusters separate like $t^{2 / 3}$. Lastly the subsystems separate from each other as $C t$. Our solutions do not have oscillatory or pulsating behavior, and all the particles separate from each other like $C t$; more specifically, particles $i$ and $j$ separate from each other as $A_{i j} t$, meaning these solutions do not cluster or form subsystems remaining any more closely together.

A further result, Theorem 8 (p. 236) in [94], again adapted for our case of no clustering improves on (5.4.5):

$$
q_{j}=a_{j} t+b_{j} \log t+c_{i}+O\left(t^{-1 / 3}\right) .
$$

For our solutions, we note that $a_{i}$ and $c_{i}$ are arbitrary, limited only that the $a_{i}$ must be distinct, and we have provided $b_{i}$ the coefficient of the the $\log t$ term in terms of the $a_{j}$ and improved the error term from $O\left(t^{-1 / 3}\right)$ to $O(\log t / t)$.

In 1976, Marchal and Saari [70] provide a framework that allow for the study of an expanding universe of clusters of particles. Their Corollary 1, adapted to our setting of particles only (since our solutions do not form clusters), may be read as follows:

Corollary. If solutions to (1.0.1) exist on $(0, \infty)$ and $R(t)=O(t)$, then

$$
q_{j}=a_{j} t+O\left(t^{2 / 3}\right), j=1,2, \cdots, N \text { and } \lim \sup r(t)>0, \text { as } t \rightarrow \infty,
$$

where the $a_{j}$ are constant vectors.

They also show that if in addition, the total energy $h \geq 0$, then at least two particles escape.

Our assumptions and techniques are radically different from the assumptions and techniques employed by Pollard, Saari, and collaborators. In particular, we have provided an existence proof of a multi-parameter set of solutions leading to total escape. We have shown that given any two sets of 3-vectors, $a_{i}$ and $c_{i}$ and masses $m_{i}, i=1, \ldots, N$ satisfying the conditions in the beginning of this Chapter, there is a set of vector functions $\delta_{i}(t), i=1, \ldots, N$, as found above so that

$$
q_{i}=a_{i} t+b_{i} \log t+c_{i}+\delta_{i}(t), \quad i=1, \ldots, N,
$$

is a solution to (1.0.1) for $t \geq t_{5}$, where the $b_{i}$ are given by (5.1.5). Moreover, each $\delta_{i}=O(\log t / t)$, improving on (5.4.7) and (5.4.8). There is no singularity, as shown above, since we have bounded every $\left\|q_{i}-q_{j}\right\|$ away from 0. Obviously, the total energy is positive, and all the particles escape (or all but one) as $t \rightarrow \infty$. Moreover, $\delta_{i}$ is approximated by a rapidly-converging series.

It is now evident that our technique can yield an approximation, as well as an asymptotic approximation, to any level of desired accuracy, of any physical quantity relevant to the evolution of our configurations of an expanding universe.

In the category of positive energy solutions it might be possible to learn more about how "many" (perhaps the measure of the set or its Baire category or at least its dimension) solutions there are in some appropriate space of masses and initial conditions (or maybe just initial conditions) for $N>4$ in the following partition of the set of all positive energy solutions:

- form clusters and subsystems like in Saari [94]

- oscillating and pulsating

- all particles separate like $A t$ - contains an open neighborhood of infinity, as described in this Chapter

- end in collision - a set of measure 0 per Saari [95]

- expand faster than At

- end in non-collision singularity

The new solutions of this Chapter being so numerous might allow us to say that other expanding solutions form a comparatively small set; e.g., maybe oscillating and pulsating motion or clustering is comparatively rare. Or maybe one could show that the union of clustering solutions with these new ones form a dense open set of initial conditions. In addition, it would be interesting to characterize initial conditions according to whether they lead to clustering or not. 
Lemaître [67] appears to have been the first to notice that the Einstein field equations of General Relativity admit solutions that expand forever. Hubble's observation [57] that many galaxies are receding from us suggests we are living in such a solution. Paraphrasing [94], the asymptotic formula

$$
\frac{\left\|\dot{q}_{i}-\dot{q}_{j}\right\|}{\left\|q_{i}-q_{j}\right\|} \sim \frac{1}{t}
$$

is the Newtonian version of Hubble's law. 


\section{CHAPTER 6}

\section{For Further Investigation}

There are several directions in which one might extend this work, as well as additional areas where this compactification might elucidate; for example,

(1) In Celestial Mechanics, one can rescale time in such a way that the singularities are transformed into critical points of the new system; for example, introducing the rescaled time $\tau$ with $\frac{d t}{d \tau}=\left(1-R^{2}\right)^{4}$ makes the equations for $\frac{d x}{d \tau}$ non-singular everywhere, and the boundary invariant in $\tau$; singularities in $q$ translate to boundary points in $x$, so singularities in $q$ would be approached as $\tau$ approaches $\infty$, but as $t \rightarrow \sigma^{-} \leq \infty$. In the polynomial setting it has been possible to compute asymptotic behavior of solutions that blow up in finite time. Here it could provide asymptotic behavior as $q$ approaches a singularity. Similarly, rescaling with a power of the product of all the $g_{i j}$ causes the collision set to become critical points instead, which leads to a new way to regularize collisions. A modest start on exploring these ideas is given in Section 1 of this Chapter.

(2) One might also learn something by compactifying in position only, rather than in position and velocity together. This is discussed briefly in Section 2 .

(3) With $\nu=1-R^{2}$, one may consider (3.1.13) as a Bernoulli equation in $\nu$

$$
\dot{\nu}=-\frac{2 \nu}{1+R^{2}} h-\frac{2 \nu^{4}}{1+R^{2}} L
$$

Some approaches to this are suggested in Section 3.

(4) If we add a forcing term to the equations of celestial mechanics, assuming that the inhomogeneity is given as a power series

$$
\sum_{m=2}^{\infty} \frac{c_{i m}}{t^{m}}
$$

convergent for sufficiently large $t$, the differential equation governing the positions $q_{i}$ of $N$ pointmasses $m_{i}, i=1, \ldots, N$ moving in $\mathbb{R}^{3}$ becomes

$$
\ddot{q}_{i}=\sum_{j \neq i} \frac{m_{j}\left(q_{j}-q_{i}\right)}{\left\|q_{i}-q_{j}\right\|^{3}}+\sum_{m=2}^{\infty} \frac{c_{i m}}{t^{m}} .
$$

Some calculations about this are given in Section 4.

(5) It might be possible to apply the integral equation and contraction mapping to the compactified problem and establish the existence of solutions to the compactified system (3.1.7) of the form

$$
x=\left(\begin{array}{c}
A_{i}+B_{i} \frac{\log t}{t}+C_{i} \frac{1}{t}+\epsilon_{i} \\
B_{N+i} \frac{\log t}{t^{2}}+C_{N+i} \frac{1}{t^{2}}+\epsilon_{N+i}
\end{array}\right)
$$

which could be uncompactified to the solution $q$.

(6) It might be useful to compactify the ambient space $\mathbb{R}^{3}$, rather than the multi-dimensional phase space, and see what happens when you compactify (1.0.1) that way.

(7) The equations of motion governing the behavior of particles influenced only by each other's charge is very similar to (1.0.1), with the additional possibility of repulsive forces. A similar approach might be useful.

(8) For differential systems of equations where the boundary is or contains an invariant set under the compactified flow, the Conley Index [21] might be applied to as another tool to study orbits that connect to the invariant set on the boundary. Hell has explored this in her dissertation and a paper $[49,50]$. 
(9) Compactifying a partial differential equation is a different kind of situation, because PDE inherently are infinite dimensional, and when you "compactify" $\mathbb{R}^{\infty}$ or $L^{2}$ and add the boundary sphere, the result is not compact. In fact, the boundary sphere isn't even compact, being diffeomorphic to the whole of $\mathbb{R}^{\infty}$ per Bessega [11]. Maybe this could be overcome by compactifying to the Hilbert Cube or by compactifying the ambient space, rather than phase space. One intuits that behavior at infinity of PDE problems might be illuminated. In particular, the Einstein equation of general relativity is a hyperbolic PDE on $\mathbb{R}^{4}$ or some other, presumably non-compact, four-dimensional manifold. Cosmologists have used other compactifications, as for example in [37]. Moreover, part of Physics' grand unification program considers a ten- or eleven-dimensional manifold, some of whose directions may already be compact; see [14] for a discussion of how a ten-dimensional universe might be a bundle over our familiar four-dimensional space-time, with a compact six-dimensional Calabi-Yau manifold as a fiber.

(10) The existence of trajectories that escape to infinity might be applicable to the aerospace engineering problem of spacecraft mission design. If so, this would be an extension or broadening of hyperbolic escape in the Kepler problem, which has been used for decades as part of the design of spacecraft missions away from Earth's vicinity. The patched-conic method of orbital mechanics "patches" together the escape hyperbola, the ellipse arc about the Sun, and an arrival hyperbola at a target body.

\subsection{Rescaled time}

If we let $g=\prod_{i<j} g_{i j}$, it is easy to see that $g^{2} L$ is bounded everywhere in the closed unit ball. We saw in Section 3.1 that $\left(1-R^{2}\right)^{6} L$ is also. These factors can be combined; $g^{m}\left(1-R^{2}\right)^{3+3 n} L$ is bounded provided $m+n \geq 1$, and we have options to rescale time in (3.1.7) and remove the singularities from the compactified system (instead of regularizing the collisions) by rescaling time with

$$
\frac{d t}{d \tau}=\frac{1}{2}\left(1+R^{2}\right) g^{m}\left(1-R^{2}\right)^{3 n} .
$$

The choice of $m=2$ and $n=0$ makes the rescaled system bounded on the closed unit ball. Letting ' denote differentiation with respect to $\tau$, we have the rescaled compactified system for $n=0$ :

$$
\begin{gathered}
x_{i}^{\prime}=\frac{1+R^{2}}{2} g^{m} x_{N+i}-\left[h+\left(1-R^{2}\right)^{3} L\right] g^{m} x_{i}, \\
x_{N+i}^{\prime}=\frac{1+R^{2}}{2}\left(1-R^{2}\right)^{3} g^{m} G_{i}-\left[h+\left(1-R^{2}\right)^{3} L\right] g^{m} x_{N+i} .
\end{gathered}
$$

For $m=3$, the resulting system is continuous on the closed unit ball, with continuous first derivatives in the interior. Thus all solutions exist for all time $\tau$, so critical points are approached only as $\tau \rightarrow \pm \infty$. For $m=2$ or 3 , it is easy to see that the rescaled system has the same critical points as before rescaling (3.1.7), as well as where the original (uncompactified) system has a collision: if particles $a$ and $b$ coincide, then $g_{a b}=0$, so $g=0 ; g^{2} L$ is bounded on the ball but discontinuous on $\Delta$, and $g^{3} L=0$ on $\Delta$; thus each $x_{i}^{\prime}=0$.

For $x_{N+i}^{\prime}$, only the sum term in (6.1.3) need be considered. Now, if $i \neq a, i \neq b$, then the sum contains no terms with denominator 0 , and $g^{3}=0$. If instead $i=a$ ( $b$ is similar), the sum over $j$ includes $b$, so $g^{3}$ times that term vanishes; the rest of the terms $(j \neq b)$ are finite, so $x_{N+i}^{\prime}$ vanishes. In summary, collisions in the uncompactified system transform to critical points in the rescaled, compactified system.

With either choice, the boundary sphere becomes invariant under the rescaled flow (and consequently so does the interior), since from (3.1.13), the additional factors of $g_{i j}$ give

$$
\frac{d R^{2}}{d \tau}=\frac{d t}{d \tau} \frac{d R^{2}}{d t}=g^{m}\left(1-R^{2}\right)\left[h+\left(1-R^{2}\right)^{3} L\right]=0
$$

on the boundary, since $g^{2} L$ is bounded on the unit ball.

Thus any trajectory in the open ball stays in the ball for all finite time, but may approach the boundary as $\tau \rightarrow \pm \infty$. We consider the system of equations restricted to the boundary sphere: 


$$
\begin{gathered}
x_{i}^{\prime}=-h g^{m} x_{i}+g^{m} x_{N+i} \\
x_{N+i}^{\prime}=-h g^{m} x_{N+i} .
\end{gathered}
$$

Critical points on the boundary fall into two categories: Type I critical points are when all $x_{N+i}=0$, in other words the set of type I critical points is the $3 N-1$ sphere given by the conditions $\sum_{i=1}^{N} x_{i}^{\dagger} x_{i}=1$ and $x_{N+i}=0$. Type II critical points are when any two particles coincide on the boundary, so the set of type II critical points is the intersection of the boundary sphere with $\Delta$.

Transforming back to the original system, we see that the set of type I critical points at $\infty$ is where the velocities are all 0 . This suggests the possibility of compactifying only in the positions and not the velocities; this notion is pursued in the sequel.

We can easily compute the Jacobian at the critical points on the boundary. Because the $\tau$-derivative of $R^{2}$ has $1-R^{2}$ as a factor, we can ignore all terms containing a factor of $\left(1-R^{2}\right)^{2}$. As a result, we can compute the Jacobian as if the equations of motion were

$$
\begin{aligned}
x_{i}^{\prime} & =-h g^{m} x_{i}+\frac{1+R^{2}}{2} g^{m} x_{N+i} \\
x_{N+i}^{\prime} & =-h g^{m} x_{N+i}
\end{aligned}
$$

The trajectories of the solutions to (6.1.5) look a lot like those of (3.2.9), except for the addition of Type II critical points, reflecting the fact that collisions can be reached in finite time $t$, but require infinite time $\tau$. It is easy to see that $A$ and $h$ satisfy

$$
\begin{aligned}
A^{\prime} & =-2 g^{2} h A \\
h^{\prime} & =g^{2}\left(A-2 h^{2}\right) .
\end{aligned}
$$

Then the $\tau$-derivative of $h / A$ is $g^{2}$. So we have

$$
\frac{A^{\prime}}{A^{2}}=-2 g^{2} \frac{h}{A}=-2 \frac{h}{A}\left(\frac{h}{A}\right)^{\prime},
$$

which we can integrate with the result again that $\frac{h^{2}-A}{A^{2}}$ is constant.

\subsection{Compactify in Position Only}

Given that we start with a second order system containing no terms in $\dot{y}$, it may be useful to compactify only in the position variables and consider the resulting second order equation, or possibly to compactify position and velocity components separately. Starting again with the force equation (1.0.1), we let $q=\kappa x$, where $\kappa=\frac{1}{1-R^{2}}$ as before, except that $x$ is the $3 N$-dimensional position vector. Then we have $\dot{x}$ in terms of $x$ and $\dot{q}$ :

$$
\dot{x}=\frac{\dot{q}}{\kappa}-\left(\nabla \kappa^{\dagger} \dot{q}\right) \frac{q}{\kappa^{2}}=\left(1-R^{2}\right) \dot{q}-2 \frac{1-R^{2}}{1+R^{2}}\left(x^{\dagger} \dot{q}\right) x,
$$

which we can differentiate again:

$$
\ddot{x}=\left(1-R^{2}\right)^{3}\left[I-\frac{2}{1+R^{2}}\left(x x^{\dagger}\right)\right] \sum_{j \neq i} \frac{m_{j}\left(x_{j}-x_{i}\right)}{g_{i j}^{3}}-\frac{2 \dot{x}^{2}}{1-R^{2}} x-4 \frac{x^{\dagger} \dot{x}}{1+R^{2}} \dot{x},
$$

where we have used the vector identity $\left(z^{\dagger} y\right) z=\left(z z^{\dagger}\right) y$, as well as the identity

$$
x^{\dagger} \dot{q}=\frac{1+R^{2}}{\left(1-R^{2}\right)^{2}} x^{\dagger} \dot{x},
$$

obtained by taking the inner product of both sides of (6.2.1) with $x$.

The system (6.2.2) is singular at the boundary and at points of collision, so it might be helpful to rescale time as above. 


\subsection{Analyze $1-R^{2}$ as a Bernoulli equation}

Multiply (6.0.1) by $k \nu^{k-1}$ to obtain

$$
\dot{\nu^{k}}=k \nu^{k-1} \dot{\nu}=-\frac{2 k \nu^{k}}{1+R^{2}} h-\frac{2 k \nu^{k+3}}{1+R^{2}} L
$$

Notice that for $k+3 \geq 6$ we know by Lemma 23 that $\frac{2 k k^{k+3}}{1+R^{2}} L$ is bounded at a collision or any other singular point of the system of ode's. Put $\nu^{k}=p^{\alpha}$. Then

$$
\begin{gathered}
\nu=p^{\alpha k^{-1}} \Longleftrightarrow p=\nu^{k \alpha^{-1}} \\
\nu^{k+3}=\left[p^{\alpha k^{-1}}\right]^{k+3}=p^{\alpha(k+3) k^{-1}} .
\end{gathered}
$$

Substituting these makes (6.3.1) an equation in the dependent variable $p$, namely,

$$
\begin{gathered}
\alpha p^{\alpha-1} \dot{p}=\frac{-2 k p^{\alpha} h}{1+R^{2}}-\frac{2 k p^{\alpha(k+3) k^{-1}}}{1+R^{2}} L \\
\dot{p}=\frac{-2 h k \alpha^{-1} p}{1+R^{2}}-\frac{2 L \alpha^{-1} k p^{\alpha(k+3) k^{-1}-\alpha+1}}{1+R^{2}} .
\end{gathered}
$$

We wish to determine the rate of decrease to zero of $1-R^{2}(t)$ as $t \rightarrow b^{-}$, where $b \leq \infty$ is the endpoint of the maximal interval of existence. To this end we consider the Bernoulli equation and replace the form of the term

$$
\frac{-2 L \alpha^{-1} k p^{\alpha(k+3) k^{-1}-\alpha+1}}{1+R^{2}} .
$$

If we set

$$
\begin{aligned}
\alpha(k+3) k^{-1}-\alpha+1 & =0 \Longrightarrow \alpha\left[(k+3) k^{-1}-1\right]=-1 \\
\alpha[(k+3)-k] & =-k=3 \alpha \Longleftrightarrow \alpha=\frac{-k}{3},
\end{aligned}
$$

then we can express

$$
\begin{aligned}
& p(t)=\left(\exp \int_{t_{0}}^{t} \frac{6 h(\eta)}{1+R^{2}(\eta)} d \eta\right) p\left(t_{0}\right)+\int_{t_{0}}^{t}\left[\exp \int_{s}^{t} \frac{-6 h(\eta)}{1+R^{2}(\eta)} d \eta\right] 6 L(s) d s \\
& =\left(\exp \int_{t_{0}}^{t} \frac{6 h(\eta)}{1+R^{2}(\eta)} d \eta\right)\left\{p\left(t_{0}\right)+\int_{t_{0}}^{t}\left[\exp \int_{s}^{t_{0}} \frac{6 h(\eta)}{1+R^{2}(\eta)} d \eta\right] 6 L(s) d s\right\} .
\end{aligned}
$$

Notice that if as expected and based on the approximate solution $h(\eta) \sim \eta^{-1}, \quad$ as $\quad \eta \rightarrow \infty$,

$$
\left.\left(\exp \int_{t_{0}}^{t} \frac{6 h(\eta)}{1+R^{2}(\eta)} d \eta\right) \sim t^{3} p\left(t_{0}\right), \quad \int_{t_{0}}^{t}\left[\exp \int_{s}^{t_{0}} \frac{6 h(\eta)}{1+R^{2}(\eta)} d \eta\right] 6 L(s) d s\right]=\mathcal{O}(1) .
$$

Therefore,

$$
\left.p(t) \sim \int_{t_{0}}^{t}\left[\exp \int_{s}^{t_{0}} \frac{6 h(\eta)}{1+R^{2}(\eta)} d \eta\right] 6 L(s) d s\right] \sim t^{3} p\left(t_{0}\right) \Longrightarrow \nu(t) \sim t^{-1}\left[p\left(t_{0}\right)\right]^{-\frac{1}{3}}
$$

The above is not the right $\alpha$. This takes the Bernoulli equation into a linear equation. However, the inhomogeneous term $\frac{-2 L \alpha^{-1} k}{1+R^{2}}$ may not be continuous or even bounded on $\left[t_{0}, b\right)$ so we change its form as follows

$$
\frac{-2 L \alpha^{-1} k p^{\alpha(k+3) k^{-1}-\alpha+1}}{1+R^{2}}=\frac{-2 L \nu^{6} \nu^{-6} \alpha^{-1} k p^{\alpha(k+3) k^{-1}-\alpha+1}}{1+R^{2}}=\frac{-2 L \nu^{6} \alpha^{-1} k p^{\alpha(k+3) k^{-1}-\alpha+1-6 \alpha k^{-1}}}{1+R^{2}},
$$

and choosing $\alpha$ such that

$$
\begin{gathered}
\alpha(k+3) k^{-1}-\alpha+1-6 \alpha k^{-1}=\alpha\left[(k+3) k^{-1}-1-6 k^{-1}\right]+1=0 \Longrightarrow \\
\alpha[(k+3)-k-6] k^{-1}+1=-3 \alpha k^{-1}+1=0 \Longleftrightarrow \alpha=\frac{k}{3} .
\end{gathered}
$$

The resulting equation for $p$ is linear; therefore a solution $p(t)$ exists on an entire interval $\left[t_{0}, b\right]$ on which the coefficients are continuous functions of $t$. 
The equation can be integrated and the solution obtained is valid on an interval $\left[t_{0}, b\right]$ where $b$ is allowed to be a singular or any collision point of the $N$ body system of equations with $b \leq \infty$. Then

$$
p(t)=\left(\exp \int_{t_{0}}^{t} \frac{-6 h(\eta)}{1+R^{2}(\eta)} d \eta\right) p\left(t_{0}\right)-\int_{t_{0}}^{t}\left[\exp \int_{s}^{t} \frac{-6 h(\eta)}{1+R^{2}(\eta)} d \eta\right] 6 \nu^{6} L(s) d s
$$

So if we assume that $t_{0}=b$ is finite and $p\left(b^{-}\right)=0$ then we have

$$
p(t)=\int_{t}^{b}\left[\exp \int_{s}^{t} \frac{-6 h(\eta)}{1+R^{2}(\eta)} d \eta\right] 6 \nu^{6} L(s) d s=\nu^{\alpha k^{-1}}=\nu^{\frac{1}{3}}
$$

Notice that

$$
\nu^{k}\left(t_{0}\right)=p^{\alpha}\left(t_{0}\right)=\left[1-R^{2}\left(t_{0}\right)\right]^{k} \Longrightarrow p\left(t_{0}\right)=\left[1-R^{2}\left(t_{0}\right)\right]^{k \alpha^{-1}} .
$$

Evidently,

or

$$
p(t) \geq 0 \Longrightarrow p\left(t_{0}\right) \geq 2\left(2+\frac{3}{k}\right)^{-1}\left(\int_{t_{0}}^{t} \exp -2\left(2+\frac{3}{k}\right)^{-1} \int_{t_{0}}^{s} \frac{h(\eta)}{1+R^{2}(\eta)} d \eta\right) L(s) d s
$$

$$
\frac{p\left(t_{0}\right)}{2\left(2+\frac{3}{k}\right)^{-1}}=\frac{\left[1-R^{2}\left(t_{0}\right)\right]^{k\left(2+\frac{3}{k}\right)^{-1}}}{2\left(2+\frac{3}{k}\right)^{-1}} \geq\left(\int_{t_{0}}^{t} \exp -2\left(2+\frac{3}{k}\right)^{-1} \int_{t_{0}}^{s} \frac{h(\eta)}{1+R^{2}(\eta)} d \eta\right) L(s) d s .
$$

The inequality (6.3.3) says that independent of the singularities manifested in $L(s)$ and/or $t=\infty$ in the integral on the right hand side of (6.3.3) is bounded. Moreover, it can be made arbitrarily small if $1-R^{2}\left(t_{0}\right)$ is small. We can also put $t_{0}=\gamma t, \quad \gamma \leq 1$.

On the other hand $p(t) \leq 1$. Hence, (6.3.2) implies that

$$
p(t)=\left(\exp -\frac{2}{2+\frac{3}{k}} \int_{t_{0}}^{t} \frac{h(\eta)}{1+R^{2}(\eta)} d \eta\right)\left\{p\left(t_{0}\right)-\frac{2}{2+\frac{3}{k}} \int_{t_{0}}^{t}\left(\exp -\frac{2}{2+\frac{3}{k}} \int_{t_{0}}^{s} \frac{h(\eta)}{1+R^{2}(\eta)} d \eta\right) L(s) d s\right\} \leq 1
$$

or that

$$
\left\{p\left(t_{0}\right)-\frac{2}{2+\frac{3}{k}} \int_{t_{0}}^{t}\left(\exp -\frac{2}{2+\frac{3}{k}} \int_{t_{0}}^{s} \frac{h(\eta)}{1+R^{2}(\eta)} d \eta\right) L(s) d s\right\} \leq \exp \frac{2}{2+\frac{3}{k}} \int_{t_{0}}^{t} \frac{h(\eta)}{1+R^{2}(\eta)} d \eta .
$$

What happens if $b$ is infinite, and no collisions at finite points took place? Then, we reconsider

$$
\begin{gathered}
\dot{\nu}=-\frac{2 \nu}{1+R^{2}} h-\frac{2 \nu^{4}}{1+R^{2}} L . \\
\dot{p}=\frac{-2 h \alpha^{-1} p}{1+R^{2}}+\frac{-2 L \alpha^{-1} p^{4 \alpha-\alpha+1}}{1+R^{2}} .
\end{gathered}
$$

In order for the Bernoulli equation to be linearized we need to choose $\alpha=-\frac{1}{3}$. We would hope to prove the following lemma.

Lemma 36. Let a solution of (3.1.7) exist on $\left[t_{0}, b\right)$ with $b$ finite. Then,

i) $1-R^{2}(t)$ exists and is well defined on $\left[t_{0}, b\right]$.

ii) $b$ is a finite singularity iff $1-R^{2}(b)=0$.

iii) If two particles $j$ and $k$ are such that they coalesce for a sequence of points $t_{l} \rightarrow b^{-}$then the derivatives blow up.

\subsection{Celestial Mechanics with a Forcing Term}

Before considering the full problem, (6.0.3), we start with the simple case of the forced 1-dimensional Kepler problem

$$
\ddot{y}=-\frac{1}{y^{2}}+\frac{c}{t^{2}},
$$

for $c \neq 0$, the solution to which we seek in the form

$$
y=a t+b+\sum_{m=1}^{\infty} \frac{d_{m}}{t^{m}} .
$$


Possibly there is a reason why one of the cases $c>0$ or $c<0$ can be neglected. Next we multiply (6.4.1) by $y^{2}$, so we can write it as

$$
y^{2}\left(\ddot{y}-\frac{c}{t^{2}}\right)=-1
$$

We will need the computations:

$$
\begin{gathered}
y^{2}=a^{2} t^{2}+2 a b t+b^{2}+2 a t \sum_{m=1}^{\infty} \frac{d_{m}}{t^{m}}+2 b \sum_{m=1}^{\infty} \frac{d_{m}}{t^{m}}+\left(\sum_{m=1}^{\infty} \frac{d_{m}}{t^{m}}\right)^{2} \\
=a^{2} t^{2}+2 a b t+b^{2}+2 a d_{1}+2 a \sum_{m=2}^{\infty} \frac{d_{m}}{t^{m-1}}+2 b \sum_{m=1}^{\infty} \frac{d_{m}}{t^{m}}+\sum_{m=2}^{\infty} \frac{1}{t^{m}} \sum_{i=1}^{m-1} d_{m-i} d_{i} \\
=a^{2} t^{2}+2 a b t+b^{2}+2 a d_{1}+\frac{2 a d_{2}+2 b d_{1}}{t}+2 a \sum_{m=2}^{\infty} \frac{d_{m+1}}{t^{m}}+2 b \sum_{m=2}^{\infty} \frac{d_{m}}{t^{m}}+\sum_{m=2}^{\infty} \frac{1}{t^{m}} \sum_{i=1}^{m-1} d_{m-i} d_{i} \\
=a^{2} t^{2}+2 a b t+b^{2}+2 a d_{1}+\frac{2 a d_{2}+2 b d_{1}}{t}+\sum_{m=2}^{\infty} \frac{1}{t^{m}}\left[2 a d_{m+1}+2 b d_{m}+\sum_{i=1}^{m-1} d_{m-i} d_{i}\right] . \\
\ddot{y}-\frac{c}{t^{2}}=-\frac{c}{t^{2}}+\sum_{m=3}^{\infty}(m-2)(m-1) \frac{d_{m-2}}{t^{m}} .
\end{gathered}
$$

The left side of (6.4.3) is the product of the last two lines,

$$
\begin{gathered}
-a^{2} c-\frac{2 a b c}{t}-\frac{\left(b^{2}+2 a d_{1}\right) c}{t^{2}}-\frac{\left(2 a d_{2}+2 b d_{1}\right) c}{t^{3}}-c \sum_{m=2}^{\infty} \frac{1}{t^{m+2}}\left[2 a d_{m+1}+2 b d_{m}+\sum_{i=1}^{m-1} d_{m-i} d_{i}\right] \\
+\left[a^{2} t^{2}+2 a b t+b^{2}+2 a d_{1}+\frac{2 a d_{2}+2 b d_{1}}{t}\right] \sum_{m=3}^{\infty}(m-2)(m-1) \frac{d_{m-2}}{t^{m}} \\
+\sum_{m=5}^{\infty} \frac{1}{t^{m}} \sum_{j=2}^{m-3}\left[2 a d_{j+1}+2 b d_{j}+\sum_{i=1}^{j-1} d_{j-i} d_{i}\right](m-j-2)(m-j-1) d_{m-j-2} .
\end{gathered}
$$

The last quantity in the first line above may be expressed

$$
-c \sum_{m=4}^{\infty} \frac{1}{t^{m}}\left[2 a d_{m-1}+2 b d_{m-2}+\sum_{i=1}^{m-3} d_{m-i-2} d_{i}\right] .
$$

As can be seen in (6.4.5) the first appearance of each $d_{m}$; that is, the lowest order term (in $t$ ) containing $d_{m}$ is the coefficient of $t^{-m}$, which arises in the middle row of (6.4.5) as

$$
m(m+1) \frac{a^{2} d_{m}}{t^{m}}
$$

which is linear in $d_{m}$, and all the $d_{m}$ can be computed. The lowest order terms of the product are

$$
-a^{2} c+\frac{2 a^{2} d_{1}-2 a b c}{t}+\frac{6 a^{2} d_{2}+2 a b d_{1}-\left(b^{2}+2 a d_{1}\right) c}{t^{2}}+\frac{12 a^{2} d_{3}+6 a b d_{2}+2\left(b^{2}+2 a d_{1}\right) d_{1}-\left(2 a d_{2}+2 b d_{1}\right) c}{t^{3}} .
$$


Since this must equal -1 for all values of $t$, the first term must equal -1 , and all the numerators must vanish; leading to equations for the coefficients of (6.4.2):

$$
\begin{aligned}
a^{2} c & =1 \\
12 a^{2} d_{3}+12 a b d_{2}+2\left(b^{2}+2 a d_{1}\right) d_{1}-2\left(a d_{2}+b d_{1}\right) c & =0 \\
6 a^{2} d_{2}+2 a b d_{1}-\left(b^{2}+2 a d_{1}\right) c & =0 \\
20 a^{2} d_{4}+24 a b d_{3}+6\left(b^{2}+2 a d_{1}\right) d_{2}+4\left(a d_{2}+b d_{1}\right) d_{1}-\left(2 a d_{3}+2 b d_{2}+d_{1}^{2}\right) c & =0 \\
m(m+1) a^{2} d_{m}+2 m(m-1) a b d_{m-1}+(m-1)(m-2)\left(b^{2}+2 a d_{1}\right) d_{m-2} & \\
+\sum_{j=2}^{m-3}\left[2 a d_{j+1}+2 b d_{j}+\sum_{i=1}^{j-1} d_{j-i} d_{i}\right](m-j-2)(m-j-1) d_{m-j-2} & \\
-\left[2 a d_{m-1}+2 b d_{m-2}+\sum_{i=1}^{m-3} d_{m-i-2} d_{i}\right] c & =0
\end{aligned}
$$

for $m>4$. These simplify

$$
\begin{aligned}
a^{2} & =1 / c \\
d_{1} & =b c \sqrt{c} \\
d_{2} & =\frac{1}{6}\left(2 b c^{3}-b^{2} c^{2}\right) \\
d_{3} & =\frac{c}{12}\left(b^{3} c+\frac{2}{3} b c^{2}-2 b^{2} c^{2}-\frac{1}{3} b^{2} c+\left[2 b^{3} c+3 b^{2} c^{2}\right] \sqrt{c}\right)
\end{aligned}
$$

where $b$ is a free parameter. In general $d_{m}$ can be expressed as a polynomial in $d_{j}, j<m$ :

$$
\begin{aligned}
m(m+1) d_{m}= & {\left[2 a d_{m-1}+2 b d_{m-2}+\sum_{i=1}^{m-3} d_{m-i-2} d_{i}\right] c^{2} } \\
& -2 m(m-1) a b c d_{m-1}-(m-1)(m-2)\left(b^{2}+2 a d_{1}\right) c d_{m-2} \\
& -c \sum_{j=2}^{m-3}\left[2 a d_{j+1}+2 b d_{j}+\sum_{i=1}^{j-1} d_{j-i} d_{i}\right](m-j-2)(m-j-1) d_{m-j-2},
\end{aligned}
$$

where we have used the fact that $a^{2}=1 / c$.

Now the case of general $N$. If we suppose that there is a power series solution

$$
q_{i}=a_{i} t+b_{i}+\sum_{m=1}^{\infty} \frac{d_{i m}}{t^{m}}
$$

where $a_{i}, b$, and $d_{m i}$ are 3 -vectors, then each denominator in (6.0.3) admits a power series. It follows that the right hand side of (6.0.3) has a power series expansion, which can be compared with that found on differentiating (6.4.6) term-by-term:

$$
\ddot{q}_{i}=\sum_{m=3}^{\infty}(m-1)(m-2) \frac{d_{i m-2}}{t^{m}} .
$$

On the other hand, we can attempt to compactify (6.0.3) and see where that goes.

6.4.1. Seeking a Power Series for $q_{i}$. The goal is to equate the right hand sides of (6.4.7) and (6.0.3) after (6.4.6) has been used to substitute for $q$ in the latter. The result is to be expressed as a power series in $1 / t$, and compared term by term to find the series coefficients in (6.4.6). We will substitute with (6.4.6) in (6.0.3), starting with the denominators:

$$
\left\|q_{i}-q_{j}\right\|^{2}=\left\|\left(a_{i}-a_{j}\right) t+b_{i}-b_{j}+\sum_{m=1}^{\infty} \frac{d_{i m}-d_{j m}}{t^{m}}\right\|^{2}
$$




$$
\begin{gathered}
=A_{i j}^{2} t^{2}+2 t\left(a_{i}-a_{j}\right)^{\dagger}\left(b_{i}-b_{j}\right)+\left\|b_{i}-b_{j}\right\|^{2}+2 t\left(a_{i}-a_{j}\right)^{\dagger} \sum_{m=1}^{\infty} \frac{d_{i m}-d_{j m}}{t^{m}} \\
+2\left(b_{i}-b_{j}\right)^{\dagger} \sum_{m=1}^{\infty} \frac{d_{i m}-d_{j m}}{t^{m}}+\left\|\sum_{m=1}^{\infty} \frac{d_{i m}-d_{j m}}{t^{m}}\right\|^{2} \\
=A_{i j}^{2} t^{2}+2 t\left(a_{i}-a_{j}\right)^{\dagger}\left(b_{i}-b_{j}\right)+\left\|b_{i}-b_{j}\right\|^{2}+2\left(a_{i}-a_{j}\right)^{\dagger}\left(d_{i 1}-d_{j 1}\right)+2\left(a_{i}-a_{j}\right)^{\dagger} \sum_{m=2}^{\infty} \frac{d_{i m}-d_{j m}}{t^{m-1}} \\
+2\left(b_{i}-b_{j}\right)^{\dagger} \sum_{m=1}^{\infty} \frac{d_{i m}-d_{j m}}{t^{m}}+\left\|\sum_{m=1}^{\infty} \frac{d_{i m}-d_{j m}}{t^{m}}\right\|^{2} \\
=A_{i j}^{2} t^{2}\left\{1+\frac{2}{A_{i j}^{2} t}\left(a_{i}-a_{j}\right)^{\dagger}\left(b_{i}-b_{j}\right)+\frac{\left\|b_{i}-b_{j}\right\|^{2}+2\left(a_{i}-a_{j}\right)^{\dagger}\left(d_{i 1}-d_{j 1}\right)}{A_{i j}^{2} t^{2}}+O\left(\frac{1}{t^{3}}\right)\right\},
\end{gathered}
$$

where $A_{i j}=\left\|a_{i}-a_{j}\right\|$. So that

$$
\begin{gathered}
\left\|q_{i}-q_{j}\right\|^{-3}=A_{i j}^{-3} t^{-3}\left\{1+\frac{2}{A_{i j}^{2} t}\left(a_{i}-a_{j}\right)^{\dagger}\left(b_{i}-b_{j}\right)+\frac{\left\|b_{i}-b_{j}\right\|^{2}+2\left(a_{i}-a_{j}\right)^{\dagger}\left(d_{i 1}-d_{j 1}\right)}{A_{i j}^{2} t^{2}}+O\left(\frac{1}{t^{3}}\right)\right\}^{-3 / 2} \\
=A_{i j}^{-3} t^{-3}\left\{1-\frac{3}{A_{i j}^{2} t}\left(a_{i}-a_{j}\right)^{\dagger}\left(b_{i}-b_{j}\right)\right\}+O\left(\frac{1}{t^{5}}\right) .
\end{gathered}
$$

Each term in the first sum of (6.0.3) can be expressed as $m_{j}$ times

$$
\begin{gathered}
A_{i j}^{-3} t^{-3}\left\{1-\frac{3}{A_{i j}^{2} t}\left(a_{i}-a_{j}\right)^{\dagger}\left(b_{i}-b_{j}\right)\right\}\left[\left(a_{j}-a_{i}\right) t+b_{j}-b_{i}+\sum_{m=1}^{\infty} \frac{d_{j m}-d_{i m}}{t^{m}}\right]+O\left(\frac{1}{t^{4}}\right) \\
=A_{i j}^{-3} t^{-2}\left\{a_{j}-a_{i}-\frac{3}{A_{i j}^{2} t}\left(a_{i}-a_{j}\right)^{\dagger}\left(b_{i}-b_{j}\right)\left(a_{j}-a_{i}\right)+\frac{b_{j}-b_{i}}{t}\right\}+O\left(\frac{1}{t^{4}}\right) \\
=\frac{a_{j}-a_{i}}{A_{i j}^{3} t^{2}}+\frac{1}{A_{i j}^{3} t^{3}}\left[I_{3}-\frac{3}{A_{i j}^{2}}\left(a_{j}-a_{i}\right)\left(a_{i}-a_{j}\right)^{\dagger}\right]\left(b_{j}-b_{i}\right)+O\left(\frac{1}{t^{4}}\right) .
\end{gathered}
$$

So we set equal the right hand sides of (6.4.7) and (6.0.3)

$$
\begin{aligned}
& \sum_{m=3}^{\infty}(m-1)(m-2) \frac{d_{i m-2}}{t^{m}}=\sum_{j \neq i} \frac{a_{j}-a_{i}}{A_{i j}^{3} t^{2}} \\
& \quad+\sum_{j \neq i} \frac{1}{A_{i j}^{3} t^{3}}\left[I_{3}-\frac{3}{A_{i j}^{2}}\left(a_{j}-a_{i}\right)\left(a_{i}-a_{j}\right)^{\dagger}\right]\left(b_{j}-b_{i}\right)+\sum_{m=2}^{\infty} \frac{c_{i m}}{t^{m}}+O\left(\frac{1}{t^{4}}\right) .
\end{aligned}
$$

Since this must be true for all values of $t$ (sufficiently large that the series all converge), the coefficients $t^{2}$ and $t^{3}$ must be equal, so we have the equations:

$$
\begin{gathered}
0=\sum_{j \neq i} \frac{a_{j}-a_{i}}{A_{i j}^{3}}+c_{i 2} \\
2 d_{i 1}=\sum_{j \neq i} \frac{1}{A_{i j}^{3}}\left[I_{3}-\frac{3}{A_{i j}^{2}}\left(a_{j}-a_{i}\right)\left(a_{i}-a_{j}\right)^{\dagger}\right]\left(b_{j}-b_{i}\right)+c_{i 3} .
\end{gathered}
$$

Note that if we sum (6.4.9) over $i$, we find that $\sum_{i=1}^{N} c_{i 2}=0$, a condition on the forcing term. Moreover, if we would keep additional terms in the power series, we would have equations for higher degree coefficients, and could in principle establish an algebraic equation for every degree. The form of the resulting equations 
suggests that if one starts with a convergent series (6.4.6) for $q_{i}$, it is possible to find $c_{i m}$ such that (6.4.6) is a solution of (6.0.3); one would need to verify that the series (6.0.2) converges.

Here we consider small $N$ cases. For the two-body problem, let $q=q_{2}-q_{1}$; then we have

$$
\ddot{q}=\left(m_{1}+m_{2}\right) \frac{q}{\|q\|^{3}}+\sum_{m=2}^{\infty} \frac{c_{2 m}-c_{1 m}}{t^{m}}
$$

which is the forced Kepler problem in $\mathbb{R}^{3}$, with $G=m_{1}+m_{2}$, instead of $G=1$. Here we repeat equations (6.4.9) and (6.4.10) for the $N=2$ case:

$$
\begin{gathered}
c_{12}=-c_{22}=-\frac{a_{2}-a_{1}}{A_{12}^{3}} \\
2 d_{11}=\frac{1}{A_{12}^{3}}\left[I_{3}+\frac{3}{A_{12}^{2}}\left(a_{2}-a_{1}\right)\left(a_{2}-a_{1}\right)^{\dagger}\right]\left(b_{2}-b_{1}\right)+c_{13} .
\end{gathered}
$$

The first of these can be met by setting $a=-c_{12} /\left\|c_{12}\right\|^{3 / 2}$, and choosing the pair $a_{1}, a_{2}$, such that $a_{2}=a_{1}+a$, and the second defines $d_{11}$, with $b_{1}$ and $b_{2}$ as yet undetermined.

Here we repeat equations (6.4.9) and (6.4.10) for the case $N=3$ :

$$
\begin{gathered}
0=\frac{a_{2}-a_{1}}{A_{12}^{3}}+\frac{a_{3}-a_{1}}{A_{13}^{3}}+c_{12} \quad 0=\frac{a_{1}-a_{2}}{A_{12}^{3}}+\frac{a_{3}-a_{2}}{A_{23}^{3}}+c_{22} \quad c_{32}=-c_{12}-c_{22} \\
2 d_{11}=\frac{1}{A_{12}^{3}}\left[I_{3}+\frac{3}{A_{12}^{2}}\left(a_{2}-a_{1}\right)\left(a_{2}-a_{1}\right)^{\dagger}\right]\left(b_{2}-b_{1}\right)+\frac{1}{A_{13}^{3}}\left[I_{3}+\frac{3}{A_{13}^{2}}\left(a_{3}-a_{1}\right)\left(a_{3}-a_{1}\right)^{\dagger}\right]\left(b_{3}-b_{1}\right)+c_{13}
\end{gathered}
$$$$
2 d_{21}=\frac{1}{A_{12}^{3}}\left[I_{3}+\frac{3}{A_{12}^{2}}\left(a_{1}-a_{2}\right)\left(a_{1}-a_{2}\right)^{\dagger}\right]\left(b_{1}-b_{2}\right)+\frac{1}{A_{23}^{3}}\left[I_{3}+\frac{3}{A_{23}^{2}}\left(a_{3}-a_{2}\right)\left(a_{3}-a_{2}\right)^{\dagger}\right]\left(b_{3}-b_{2}\right)+c_{23}
$$

$$
2 d_{31}=\frac{1}{A_{13}^{3}}\left[I_{3}+\frac{3}{A_{13}^{2}}\left(a_{1}-a_{3}\right)\left(a_{1}-a_{3}\right)^{\dagger}\right]\left(b_{1}-b_{3}\right)+\frac{1}{A_{23}^{3}}\left[I_{3}+\frac{3}{A_{23}^{2}}\left(a_{2}-a_{3}\right)\left(a_{2}-a_{3}\right)^{\dagger}\right]\left(b_{2}-b_{3}\right)+c_{33} .
$$

If we sum the last three equations, we find that

$$
2 d_{11}+2 d_{21}+2 d_{31}=c_{13}+c_{23}+c_{33} .
$$

We can solve the $N=3$ equations, beginning with (6.4.13), which we solve for the differences

$$
\frac{a_{2}-a_{1}}{A_{12}^{3}}=\frac{1}{3}\left(-c_{12}+c_{22}\right) \quad \frac{a_{3}-a_{1}}{A_{13}^{3}}=\frac{1}{3}\left(-c_{12}+c_{32}\right) \quad \frac{a_{3}-a_{2}}{A_{23}^{3}}=\frac{1}{3}\left(-c_{22}+c_{32}\right),
$$

which works if the numerators on the left are equal to the vectors on the right divided by the $3 / 2$ power of the magnitude of the vector on the right; e.g.,

$$
a_{2}-a_{1}=\sqrt{3} \frac{-c_{12}+c_{22}}{\left\|-c_{12}+c_{22}\right\|^{3 / 2}} .
$$

We only need the differences in the $a_{i}$ to determine the $d_{i 1}$ from (6.4.14), with the $b_{i}$ unconstrained.

6.4.2. Compactifying the Forced N-Body Problem. In this Section, we develop the equations of motion for the compactified N-body problem and show that the compactification does not introduce any critical points in the open unit ball, which means that the topological structures of the flows on the open ball and on all of Euclidean space should be identical. We show that if a trajectory experiences a singularity as $t \rightarrow \sigma^{-}$, then the compactified trajectory approaches the boundary sphere. The Section closes with the behavior of the magnitude of the compactified $6 N$-vector, which quantity satisfies a Bernoulli equation.

We make the second-order equation for the $q_{i}$ (1.0.1) into a $6 N$-dimensional system in the usual way: for $i=1, \ldots, N$, let $y_{i}=q_{i}$, and $y_{N+i}=\dot{q}_{i}$, where for each $i, y_{i}$ and $y_{N+i}$ are vectors in $\mathbb{R}^{3}$. Then we can express the system as $\dot{y}=f(y, t)$, with the right hand side serving to define $f(y, t)$ :

$$
\begin{aligned}
\dot{y}_{i} & =y_{N+i} \\
\dot{y}_{N+i} & =\sum_{j \neq i} \frac{m_{j}\left(y_{j}-y_{i}\right)}{\left\|y_{i}-y_{j}\right\|^{3}}+\sum_{m=2}^{\infty} \frac{c_{i m}}{t^{m}} .
\end{aligned}
$$


Our compactified version of the original system of equations will be obtained on substituting $y=\frac{x}{1-x^{\dagger} x}$. All of $\mathbb{R}^{6 N}$ is mapped to the open unit ball, and the pre-image of its boundary $\mathbb{S}^{6 N-1}$ is an ideal set bounding $\mathbb{R}^{6 N}$, which may be identified with all directions at infinity. Compactifications in general, and this one in particular, make it possible to augment the conventional set of solutions of a differential system with an ideal set of solutions $y \equiv \infty$. Their geometric realization is given by trajectories on $\mathbb{S}^{6 N-1}$ viewed as the boundary sphere of the unit ball in $\mathbb{R}^{6 N}$. One should expect these ideal trajectories to be solutions to simplified systems of differential equations. Indeed, these ideal solutions, as shown in this Chapter, provide a wealth of information on the solutions of $\dot{y}=f(y)$ for $\|y\|$ large.

As in Chapter 2, we compactify with $y=\kappa x$, with $\kappa=\frac{1}{1-R^{2}}$, where $R=\|x\|$. Then

$$
\dot{x}=\frac{\dot{y}}{\kappa}-(\nabla \kappa)^{\dagger} \dot{y} \frac{y}{\kappa^{2}}=\kappa^{-1}\left[f(y)-(\nabla \kappa)^{\dagger} f(y) \frac{y}{\kappa}\right] .
$$

Squaring both sides of the transformation equation $y=\frac{x}{1-R^{2}}$ gives $r^{2}=\frac{R^{2}}{\left(1-R^{2}\right)^{2}}$, which leads to $R^{2}=$ $\left(2 r^{2}+1 \pm \sqrt{1+4 r^{2}}\right) / 2 r^{2}$, with $r=\|y\|$. To ensure $R \in[0,1]$, we choose the negative sign. Then $\kappa=$ $\frac{1}{1-R^{2}}=\frac{1}{2}\left(1+\sqrt{1+4 r^{2}}\right)$. The last equation can be solved for the radical: $\sqrt{1+4 r^{2}}=\frac{1+R^{2}}{1-R^{2}}$, so that

$$
\nabla \kappa=\frac{2 y}{\sqrt{1+4 r^{2}}}=\frac{2 y\left(1-R^{2}\right)}{1+R^{2}}=\frac{2 x}{1+R^{2}} .
$$

Then

$$
(\nabla \kappa)^{\dagger} f(y)=\frac{2}{1+R^{2}} x^{\dagger} f(y)=\frac{2}{1+R^{2}} \sum_{k=1}^{N} x_{N+k}^{\dagger}\left(\frac{x_{k}}{1-R^{2}}+\left(1-R^{2}\right)^{2} \sum_{j \neq k} \frac{m_{j}\left(x_{j}-x_{k}\right)}{\left\|x_{k}-x_{j}\right\|^{3}}+\sum_{m=2}^{\infty} \frac{c_{i m}}{t^{m}}\right),
$$

so that the evolution of the compactified system in the open unit ball $\mathbb{B}^{6 N}$ in $\mathbb{R}^{6 N}$ is governed by

$$
\begin{aligned}
\dot{x_{i}}= & x_{N+i}-\frac{2}{1+R^{2}} \sum_{k=1}^{N} x_{N+k}^{\dagger}\left(x_{k}+\left(1-R^{2}\right)^{3} \sum_{j \neq k} \frac{m_{j}\left(x_{j}-x_{k}\right)}{\left\|x_{k}-x_{j}\right\|^{3}}+\sum_{m=2}^{\infty} \frac{c_{i m}}{t^{m}}\right) x_{i} \\
\dot{x}_{N+i}= & \left(1-R^{2}\right)^{3} \sum_{k \neq i} \frac{m_{k}\left(x_{k}-x_{i}\right)}{\left\|x_{i}-x_{k}\right\|^{3}}+\left(1-R^{2}\right) \sum_{m=2}^{\infty} \frac{c_{i m}}{t^{m}} \\
& -\frac{2}{1+R^{2}} \sum_{k=1}^{N} x_{N+k}^{\dagger}\left(x_{k}+\left(1-R^{2}\right)^{3} \sum_{j \neq k} \frac{m_{j}\left(x_{j}-x_{k}\right)}{\left\|x_{k}-x_{j}\right\|^{3}}+\sum_{m=2}^{\infty} \frac{c_{i m}}{t^{m}}\right) x_{N+i} .
\end{aligned}
$$

I also looked at the forced problem restricted to the boundary; I get the same critical point set, but not exact formulas for solutions on the boundary, like we have in the non-forced case. 


\section{Bibliography}

[1] Mark J. Ablowitz \& Harvey Segur, Solitons and the Inverse Scattering Transform, SIAM, 1981.

[2] L. A. Ahlfors, Complex Analysis, McGraw-Hill, New York, N.Y., 1979.

[3] A. A. Andronov, E. A. Leontovich, I. I. Gordon and A. L. Maier, "Qualitative Theory of Second-order Dynamic Systems," Wiley, New York, 1973.

[4] J. C. Artés, R. E. Kooij and J. Llibre, "Structurally stable quadratic vector fields," Mem. Amer. Math. Soc. 134 (1998), no. 639, viii+108 pp.

[5] J.C. Artés, J. Llibre, J.C. Medrado, Nonexistence of Limit Cycles for a Class of Structurally Stable Quadratic Vector Fields, Discrete and Continuous Dynamical Systems, Volume 17, Number 2, February 2007.

[6] J. C. Artés, J. Llibre, N. Vulpe, Singular points of quadratic systems: a complete classification in the coefficient space, Internat. J. Bifur. Chaos Appl. Sci. Engrg. 18 (2008), no. 2, 313-362.

[7] R. Barboza, Diffusive Synchronization of Hyperchaotic Lorenz Systems, Mathematical Problems in Engineering, vol. 2009, Article ID 174546, 2009. doi:10.1155/2009/174546

[8] N. Bautin, On periodic solutions of a system of differential equations, Prikl. Math. Meh., 18 (1954), 128.

[9] E. Belbruno, On simultaneous double collision in the collinear four-body problem, J. Differential Equations, 52 (1984), 415-431.

[10] I. Bendixson, Sur les courbes définies par des équations differérentielles, Acta Mathematica, 24 (1901) 1-88.

[11] C. Bessega, "Every infinite-dimensional Hilbert space is diffeomorphic with its unit sphere," Bull. Acad. Polon. Sci XIV (1966), 27-31

[12] G. Birkhoff, Dynamical Systems, second edition, American Mathematical Society, 1966.

[13] M. Bohlin, Astronomiska Jakttagelser och undersekningar, Band 9, 1908.

[14] P. Candelas, G. Horowitz, A. Strominger, E. Witten, "Vacuum configurations for superstrings", Nuclear Physics B 258 (1985), 46-74.

[15] Chen, Guang Qing; Liang, Zhao Jun, "Affine classification for the quadratic vector fields without the critical points at infinity," J. Math. Anal. Appl., 172 (1993), 62-72.

[16] Chen Xiang-yan, On generalized rotated vector fields, Nanjing Daxue Xuebao (Nat. Sci.), 1 (1975), 100-108.

[17] J. Chazy, "Sur l'allure du mouvement dans le prolème de trois corps quand le temps croît indéfiniment," Annales Scientifiques de l'École Norm., 39 (1922), 29-13

[18] 0 C. Chicone and J. Sotomayor, "On a Class of Complete Polynomial Vector Fields in the Plane," Journal of Differential Equations 61 (1986), 398-418.

[19] A. Cima and J. Llibre, "Bounded Polynomial Vector Fields," Transactions of the American Mathematical Society, Vol. 318 (1990), 557-579.

[20] E. Coddington \& N. Levinson, Theory of Ordinary Differential Equations, McGraw-Hill, 1955.

[21] C. Conley, Isolated Invariant Sets and the Morse Index, CBMS Regional Conf. Ser. Math. 38, 1978.

[22] B. Coomes, The Lorenz System does not have a Polynomial Flow, Journal of Differential Equations 82 (1989) 386-407.

[23] W. Coppel, A Survey of Quadratic Systems, Journal of Differential Equations 2 (1966) 293-304.

[24] J. Coste, J. Peyraud and P. Coullet, Asymptotic behavior in the dynamics of competing Species, SIAM J. APPL. Math. Vol. 36, No. 3, (1979), 516-543.

[25] G.F.D. Duff, Limit-cycles and rotated vector fields, Ann.of Math., 57 (1953), 15-31.

[26] F. Diacu, "The Solution of the n-body Problem," Mathematical Intelligencer, 18 (1996) 66-70.

[27] F. Dumortier, J. Llibre and J. Artés, Qualitative theory of planar differential systems, Universitext, SpringerVerlag, Berlin, 2006, xvi+298 pp.

[28] M. Eastham, The Asymptotic Solution of Linear Differential Systems, Oxford, 1989.

[29] M. S. ElBialy, Collision-ejection manifold and collective analytic continuation of simultaneous binary collisions in the planar n-body problem, J. Math. Analysis and Applications, 203 (1996), 55-77.

[30] U. Elias, H. Gingold,"Asymptotic approximation for matrix differential equations and applications," Differential Integral Equations, 10 (1997), 137-152.

[31] U. Elias, H. Gingold,"More on asymptotic approximation for matrix differential equations," J. Math. Analysis and Applications, 230 (1999), 97-111.

[32] U. Elias, H. Gingold,"Effects of varying nonlineariy and their singular perturbation flavour," J. Math. Analysis and Applications, 248 (2000), 309-326.

[33] U. Elias, H. Gingold,"A method for asymptotic integration of almost diagonal systems," Asymptotic Analysis, 29 (2002), 343-357. 
[34] U. Elias, H. Gingold, "A framework for asymptotic integration of differential systems," Asymptotic Analysis 35 (2003), 281-300.

[35] U. Elias, H. Gingold, Critical points at infinity and blow up of solutions of autonomous polynomial differential systems via compactification, Journal of Mathematical Analysis and Applications, 318 (2006), no. 1, 305-322.

[36] A. Yu. Fishkin, On the number of limit cycles of planar quadratic vector fields. (Russian) Dokl. Akad. Nauk 428 (2009), no. 4, 462-464.

[37] R. Geroch, "Asymptotic Structure of Space-time," from the book Asymptotic Structure of Space-time, F. P. Esposito \& L. Witten, eds, Plenum, New York, 1977.

[38] H. Gingold, Approximation of unbounded functions via compactification, Journal of Approximation Theory 131 (2004), 284-305.

[39] Y. Gingold, H. Gingold, Geometric Properties of a Family of Compactifications, Balkan Journal of Geom. Appl 12 (2007), 44-55.

[40] H. Gingold and D. Solomon, "The Lorenz System has a Global Repeller at Infinity," Journal of Nonlinear Mathematical Physics, 18 (2011), 183-189.

[41] H. Gingold and D. Solomon, "On Completeness of Quadratic Systems," Nonlinear Analysis, 74 (2011) 4234-4240.

[42] H. Gingold and D. Solomon, "More Compactification for Differential Systems," Advances in Pure Mathematics, 3 (2013), 190-203. doi: 10.4236/apm.2013.31A027.

[43] H. Gingold and D. Solomon, "Celestial mechanics solutions that escape," Journal of Differential Equations, 263 (2017), 1813-1842, http://dx.doi.org/10.1016/j.jde.2017.03.030.

[44] H. Gingold and A. Tovbis, "Solutions of Finitely Smooth Nonlinear Singular Differential Equations and Problems of Diagonalization and Triangulation," SIAM Journal of Mathematical Analysis, 29 (1998), 757-778.

[45] I. Gradshteyn \& I. Ryzhik, Table of Integrals, Series, and Products, Academic Press, 1980.

[46] J. Guckenheimer \& P. Holmes, Nonlinear Oscillations, Dynamical Systems and Bifurcations of Vector Fields, Springer-Verlag New York, 1983.

[47] J. Harlim \& A. J. Majda, Filtering Nonlinear Dynamical Systems with Linear Stochastic Models, Nonlinearity 21 (2008) 1281-1306.

[48] P. Hartman, Ordinary Differential Equations, 2nd edition, Birkhäuser, 1982.

[49] J. Hell, Conley Index at Infinity, a PhD dissertation submited to the Free University of Berlin, 2010.

[50] J. Hell, "Conley Index at Infinity," https://arxiv.org/abs/1103.5335v2, 2012.

[51] Einar Hille, A Note on Quadratic Systems, Proceedings of the Royal Society of Edinburgh, Section A, 72 (1974), 17-37.

[52] E. Hille, Analytic Function Theory, Chelsea Publishing Company, New York, N.Y., 1982.

[53] K. Hoffman \& R. Kunze, Linear Algebra, 2nd ed, Prentice Hall, Englewood Cliffs, New Jersey, 1971.

[54] Hsieh, Po-Fang \& Sibuya, Yasutaka, Basic Theory of Ordinary Differential Equations, Universitext. SpringerVerlag, New York, 1999.

[55] S-B, Hsu, S. Hubbell \& P. Waltman, A contribution to the theory of competing predators, Ecological Monographs 48 (1979), 337-349.

[56] D.D. Hua, L. Cairó, M.R. Feix, K.S. Govinder \& P.G.L. Leach, Connection between the existence of first integrals and the Painlevé Property in Lotka-Volterra and Quadratic Systems, Proc Roy Soc Lond 452 (1996), 859-880.

[57] E. Hubble, "A relation between distance and radial velocity among extra-galactic nebulae," Proceedings of the National Academy of Sciences, 15 (1929) 168-73.

[58] Yu. S. Ilýashenko, "Finiteness Theorems for Limit Cycles", Translated from the Russian by H. H. McFaden. Translations of Mathematical Monographs, 94. American Mathematical Society, Providence, RI, 1991. x $+288 \mathrm{pp}$.

[59] F. John, Partial Differential Equations, 4th ed, Springer-Verlag, 1982.

[60] D. Jordan \& P. Smith, Nonlinear Ordinary Differential Equations: An Introduction for Scientists and Engineers, 4th edition, Oxford Texts in Applied and Engineering Mathematics, 2007.

[61] T. Kato, A Short Introduction to Perturbation Theory for Linear Operators, Springer-Verlag, 1982.

[62] V. Kozlov \& V. Palamodov, "On Asymptotic Solutions of the Equations of Classical Mechanics," Soviet Math Dokl., 25 (1982), 335-339.

[63] V. Kozlov, "Asymptotic Solutions of Equations of Classical Mechanics," Prikl. Matem. Mekhan., 46 (1982), $573-577$.

[64] A. N. Kuznetsov, "Differentiable solutions to degenerate systems of ordinary equations," Functional Analysis and its Applications, 6 (1972), 119-127.

[65] P.G.L. Leach \& J. Miritzis, Competing Species: Integrability and Stability, Journal of Nonlinear Mathematical Physics, 11 (2004) 123-133.

[66] S. Lefschetz, Differential equations: Geometric theory, Dover, New York, 1977.

[67] G. Lemaître, "Un univers homogène de masse constante et de rayon croissant rendant compte de la vitesse radiale des nébuleuses extra-galactiques". Annales de la Société Scientifique de Bruxelles A (1927) 49-59.

[68] E. N. Lorenz, Deterministic Nonperiodic Flow, Journal of the Atmospheric Sciences 20 (1963), $130-141$.

[69] Luo, Albert C. J., Global Transversality, Resonance, and Chaotic Dynamics, World Scientific Publishing Co. Pte. Ltd., Hackensack, NJ, 2008.

[70] C. Marchal \& D. Saari, "On the final evolution of the $n$-body problem," Journal of Differntial Equations, 20 (1976) 150-186.

[71] J. N. Mather and R. McGehee, Solution for collinear four-body problem which becomes unbounded in finite time, pages 573-597 in Dynamical Systems, Theory and Applications, Battelle Rencontres (Seattle 1974), Lecture Notes in Physics, Vol 38, Springer- Verlag (1975). 
[72] R. McGehee, Triple collision in collinear three-body problem, Invent. Math., 27 (1974), 191-227.

[73] R. McGehee, "Von Zeipel's theorem on singularities in celestial mechanics," Expo. Math. 4 (1986), 335-345.

[74] G. Meisters, "Polynomial Flows on $\mathbb{R}^{n}$," Proceedings of the Semester on Dynamical Systems, Autumn 1986, at the Stefan Banach International Mathematics Center, ul. Mokotowska 25, Warszawa, Poland.

[75] A. Mingarelli \& C. Mingarelli, "Conjugate points in the gravitational $n$-body problem," Celestial Mechanics and Dynamical Astronomy, 91 (2005), 391-401.

[76] R. Moeckel, "Central Configurations" on Scholarpedia, 2014, doi:10.4249/scholarpedia.10667.

[77] R. Moeckel \& R Montgomery, Symmetric Regularization, Reduction and Blow-up of the Planar Three-body Problem, Pacific Journal of Mathematics, 262 (2013), 129-189.

[78] J.D. Murray, Mathematical Biology, Biomathematics Texts 19, Springer-Verlag, 1989.

[79] F. Olver, Asymptotics and Special Functions, Academic Press, 1974.

[80] P. Painlevé, Lecons sur la Théorie Analytique des Equations Différentielles, Hermann, Paris, 1897.

[81] K. Pepkin \& W. Taylor, Senior Wonders: People Who Achieved Their Dreams After Age 60, 2014.

[82] L. Perko, Rotated vector fields and the global behavior of limit cycles for a class of quadratic systems in the plane, J. Differential Equations, 18 (1975), 63-86.

[83] L. Perko, Differential Equations and Dynamical Systems, 3rd edition, Texts in Applied Mathematics 7, SpringerVerlag, 2001

[84] E.L. Pianka, Competition and niche theory in: Theoretical Ecology: Principles and Applications, May RM, Blackwells, Oxford, 167-196, 1981.

[85] H. Poincaré, Mémoire sur les courbes définies par une équation differérentielle, Journal Mathématiques, 7 (1881) 375-422.

[86] H. Pollard, The Behavior of Gravitational Systems, Journal of Mathematics and Mechanics, 17 (1967) 601-611.

[87] H. Pollard, Celestial Mechanics, Carus Mathematical Monographs, 18, Mathematical Association of America, 1976.

[88] H. Pollard \& D. Saari, Singularities of the n-Body Problem I, Arch. Rational Mech. Anal., 30 (1968), 263-269.

[89] H. Pollard \& D. Saari, Singularities of the n-Body Problem II, Inequalites II, Academic Press, 1970, $255-259$.

[90] H. Pollard \& D. Saari, Escape from a Gravitational System of Positive Energy, Celestial Mechanics, 1 (1970) 347-350.

[91] J.W. Reyn, A bibliography of the qualitative theory of quadratic systems of differential equations in the plane, 3rd ed., Report of the Faculty of Technical Math. and Infor. 94-02, Delft, 1994.

[92] R. K. W. Roeder, "On Poincaré's fourth and fifth examples of limit cycles at infinity," Rocky Mountain J. Math, 33 (2003), 1057-1082.

[93] D. Saari, On Oscillatory Motion in the Problem of Three Bodies, Celestial Mechanics, 1 (1970) 343-346.

[94] D. Saari, Expanding Gravitational Systems, Transactions of the American Mathematical Society, 156 (1971) 219240.

[95] D. Saari, "Improbability of collisions in Newtonian gravitational systems II," Transactions of the American Mathematical Society, 181 (1972), 351-368.

[96] D. Saari, "A global existence theorem for the four body problem," Bulletin of the American Mathematical Society, 82 (1976), 743-744.

[97] D. Saari, "A global existence theorem for the four-body problem of Newtonian mechanics," Journal of Differential Equations, 26 (1977), 80-111.

[98] D. G. Saari, The manifold structure for collision and for hyperbolic-parabolic orbits in the n-body problem, J. Differential Equations, 55 (1984), 300-329.

[99] D. Saari \& F. Diacu, "Superhyperbolic expansion, non-collision singularities and symmetry configurations," Celestial Mechanics and Dynamical Astronomy, 60 (1994) 91-98.

[100] D. Saari \& Z. Xia, "Singularities in the Newtonian $n$-body Problem," Hamiltonian Dynamics and Celestial Mechanics, Contemporary Math 198 (1996), AMS, 21-30.

[101] G. Sansone and R. Conti, Non-linear differential equations, Pergamon Press, Oxford, 1964.

[102] D. Schlomiuk and N. Vulpe, "The Full Study of Planar Quadratic Differential Systems Possessing a Line of Singularities at Infinity," J Dyn Diff Equat 20 (2008), 737-775.

[103] C. Simo and E. Lacomba, Regularization of simultaneous binary collisions in the n-body problem, J. Differential Equations, 98 (1992), 241-259.

[104] C. L. Siegel, Der Dreiesto $\beta$, Ann. Math. 42 (1941), 127-168.

[105] C. L. Siegel and J.K. Moser, Lectures on Celestial Mechanics, Springer-Verlag, 1971.

[106] S. Shutǒglu and D. Viswanath, Complex Singularities and the Lorenz Attractor, SIAM Review, 52 (2010), $294-314$. http://dx.doi.org/10.1137/090753474

[107] C. Sparrow, The Lorenz Equations: Bifurcations, Chaos, and Strange Attractors, Applied Mathematical Sciences, 41, Springer-Verlag, 1982.

[108] K. F. Sundman, "Mémoire sur le problème des trios corps," Acta. Math., 36 (1913), 105-179.

[109] M. Tabor \& J. Weiss, "Analytic structure of the Lorenz system," Physical Review A 24 (1981) 2157-2167.

[110] A. Tovbis, "Normal forms of "near similarity' transformations and linear matrix equations," Linear Algebra and its Applications 317 (2000) 13-40.

[111] C. Vidal \& P. Gomez, "An extension of the Poincaré compactification and a geometric interpretation," Proyecciones 22 (2003), 161-180.

[112] H. von Zeipel, Sur les singularitiés du problème des n corps, Arkiv fur Mat., Astr. Fysik 4 (1908), 1-4. 
[113] Q. D. Wang, The global solution of N-body problem, Celest. Mech., 50 (1991), 73-88.

[114] W. Wasow, Asymptotic Expansions for Ordinary Differential Equations, Wiley, 1965.

[115] S. Willard, General Topology, Addison-Wesley, Reading, MA, 1970.

[116] A. Wintner, The Analytical Foundations of Celestial Mechanics, Princeton University Press, 1941.

[117] Z. Xia, "The Existence of Non-collision Singularities in Newtonian Systems," Annals of Mathematics, 135 (1992), 411-468.

[118] Ye Yanqian, Theory of Limit Cycles, Amer. Math. Soc., Providence, R.I., 1986. 